\title{
Systematics of lower Cyclorrhaphan Diptera, with an emphasis on Syrphidae
}

\author{
by \\ Andrew Young \\ A thesis submitted to the Faculty of Graduate and Postdoctoral Affairs in partial \\ fulfillment of the requirements for the degree of \\ Doctor of Philosophy \\ in \\ Biology \\ Carleton University \\ Ottawa, Ontario
}

(C) 2018

Andrew Young 


\section{Abstract}

This doctoral thesis is presented in three chapters. The first chapter is a phylogenetic analysis of the lower Cyclorrhaphan (Insecta: Diptera) families of Diptera. These families form a paraphyletic grade, and have been a long-standing problematic assemblage of the Dipteran tree of life. We attempted to bring resolution to this assemblage of families using a combination of transcriptomic and anchored hybrid enrichment data from 43 ingroup taxa. Results were largely concordant with existing molecular phylogenetic efforts, but Lonchopteridae remains problematic to place. The second chapter of the thesis is a more detailed phylogenetic analysis of a single lower Cyclorrhaphan family, the Syrphidae. This chapter has also been published as an open-access paper in BMC Evolutionary Biology. Subfamilial boundaries within Syrphidae are a known problem, and there has been little consensus on how to treat the paraphyletic subfamily Eristalinae. We used a novel anchored hybrid enrichment probe kit to produce a 30 species phylogeny of Syrphidae with strong support values across the entire tree. As expected, a paraphyletic Eristalinae was recovered. Our sampling was not rigorous enough to produce a stable revised classification, but we hope this chapter will act as a starting point for a future reclassification of the subfamily. This chapter has been published as an open-access paper in BMC Evolutionary Biology. The final chapter of the thesis was also Syrphidae-focused, with a taxonomic revision of the Australian Psilota. The revision is based on a combination of Cytochrome $c$ Oxidase I DNA barcodes and morphological analysis. The number of known Australian Psilota species was increased from 14 to 30, with two acknowledged species complexes 
that should be revisited in the future. All species are fully described and illustrated, with external habitus and male genitalia photographs. 


\section{Preface:}

The first and third chapters of this thesis are unpublished manuscripts. The second chapter, "Anchored enrichment dataset for true flies (order Diptera) reveals insights into the phylogeny of flower flies (family Syrphidae)" is published in BMC Evolutionary Biology. This publication is here reproduced in full (with the exception of supplementary tables, where a link to the freely-available publication is instead provided due to their length), and was published as an open-access paper with the authors retaining full copyright of the article. This was a co-authored publication, and full contributions of all authors are listed at the beginning of the chapter. The full bibliographic information for this chapter is as follows: "Young, A. D., A. R. Lemmon, J. H. Skevington, X. Mengual, G. Ståhls, M. Reemer, K. Jordaens, S. Kelso, E. M. Lemmon, M. Hauser, M. De Meyer, B. Misof and B. Wiegmann (2016). "Anchored enrichment dataset for true flies (order Diptera) reveals insights into the phylogeny of flower flies (family Syrphidae)." BMC Evolutionary Biology 16(1): 143."

As one of the lead authors on this paper, I was fully involved in setting up and conducting the research, obtaining data, and analyzing results, a well as preparing and writing the material presented in the thesis. Specifically, I have expressed an interested in Syrphidae phylogeny to Dr. Jeff Skevington since at least 2012, and have been interested in approaching it using molecular methods. I was responsible for identifying all Syrphidae voucher specimens used in the analysis, and I analyzed and compared the phylogenetic tree generated from our dataset to all published syrphid phylogenies. All parts of the second chapter were written by me except for 
the original methods section, which was written by Alan and Emily Lemmon (who pioneered this specific target enrichment technique). Full details of all author contributions are noted at the beginning of chapter 2 .

Supervisor's statement: All of the above information is correct. Andrew has been involved in discussions of Syrphidae phylogenetics with my lab since completing his M.Sc. in 2012, and was fully involved in conducting the research, obtaining data, and analyzing the results of the phylogenetic analysis, as well as preparing and writing the material provided in the thesis (except where noted above). 


\section{Acknowledgements}

Friends, family, and colleagues too numerous to mention were instrumental in the successful completion of this thesis, many of whom were invaluable to me as both emotional and academic support.

On an academic front, I would like to thank the 1KITE and North Carolina State University labs for providing sequences used in the first chapter of the thesis, and Oliver Niehuis and Berhard Misof for teaching the bioinformatics course that was invaluable to me during data analysis. Wouter van Steenis and Chris Thompson's insights into Australian Psilota taxonomy were also invaluable, especially when I was first starting out with the group. Keith Bayless, Chris Thompson, Trevor Burt, Ximo Mengual, and the entire Australian Museum, Queensland Museum, and Australian National Insect Collection Diptera units all kindly hosted me during lab visits at various points in my thesis, some of them in their own homes. Jim O'Hara, Jeff Cumming, Brad Sinclair, Michelle Locke, Owen Lonsdale, Scott Kelso, Scott Brooks, Shannon Henderson, A.J. Fleming, and everyone else who shows up in the CNC Diptera Unit: our conversations were always enlightening, about Diptera and many other things. Joel Kits helped me run Trinity on the CNC supercomputer cluster for Chapter 1 and provided me with excellent thesis-related advice. In Taiwan, Wouter van Steenis, Jeroen van Steenis, Menno van Zuijen, Chris Palmer, and Axel Ssymank taught me more about collecting Syrphidae in three weeks than I could have learned in an entire degree on my own, while 吳宗

學 (“Bill”) was an invaluable local guide and keen collector. Morgan Jackson, Miles 
Zhang, and Adam Jewiss-Gaines are all close friends who have been working towards their doctorates simultaneously to me, and all of them have been invaluable sounding boards and sources of motivation during this entire degree. Victoria Nowell, Sebastian Namek, and Nadia Boukina were all summer students when I worked with them, and all of them saved me from mountains of databasing and other invaluable, but time consuming tasks. My external advisory committee, Julian Starr and Andrew Simons, as well as my external defence committee members, Vazrick Nazari and Nicolas Rodrigue were invaluable and provided much useful critique during and after the defence process. Finally, I'd like to thank my supervisors: Jeff Skevington for teaching me much of what I know about Syrphidae and for always providing me the opportunities I needed to succeed, and Jeff Dawson, for always being understanding of entomological complications and helping me navigate Carleton University.

On a personal note, I'd like to thank all the friends who have supported me along the way, whether or not they understood or cared about whatever academic mishaps I ranted about to them. Thanks to Steve Marshall, for going out on a limb and hiring me as a M.Sc. student with no funding. An extra special thanks to my parents and brother for always being supportive of me, even when they'd rather I didn't bring so many live animals into the house. I'd particularly like to thank my maternal grandfather, Peter McQueen, who passed away while I was collecting data in Australia. Most of all, I'd like to thank Aislinn Wyatt, my partner and better half, for always supporting me, believing in me, and generally being awesome. Hail Satan. 


\section{Table of Contents}

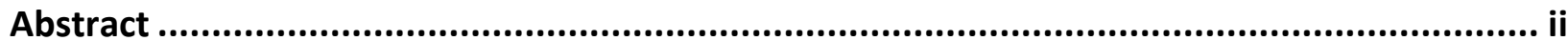

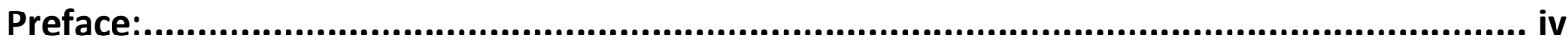

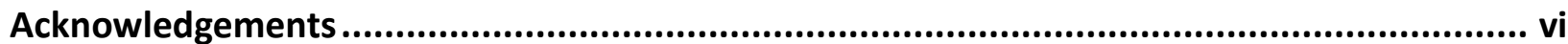

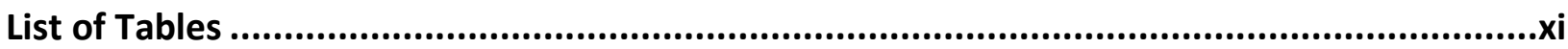

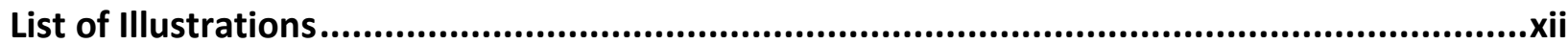

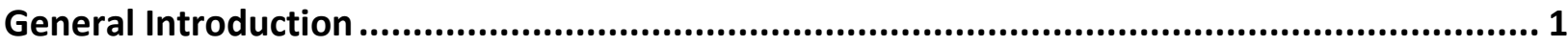

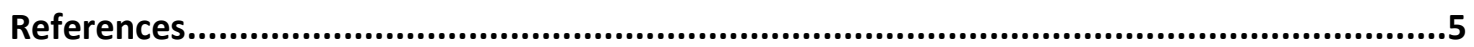

Chapter 1: Phylogenetics of lower Cyclorrhapha using transcriptome and hybrid

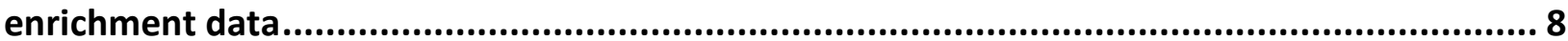

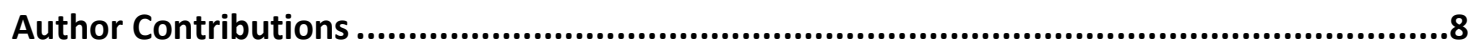

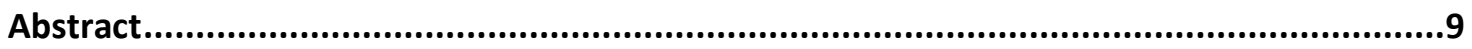

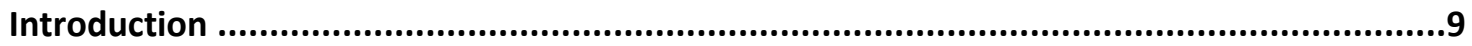

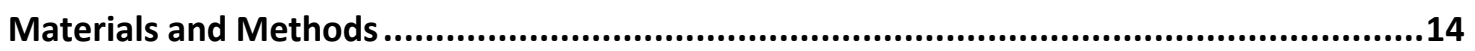

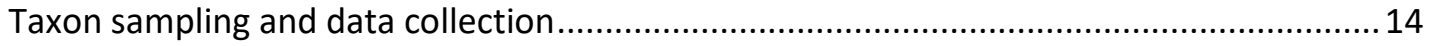

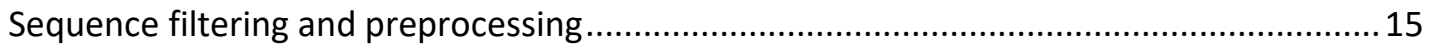

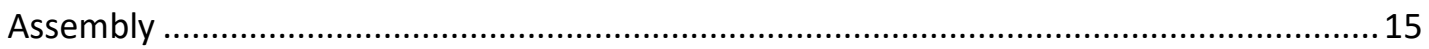

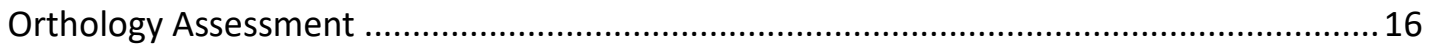

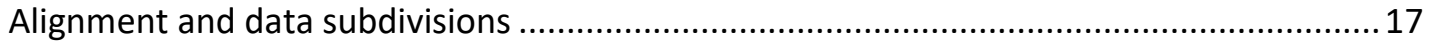

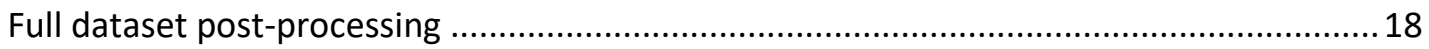

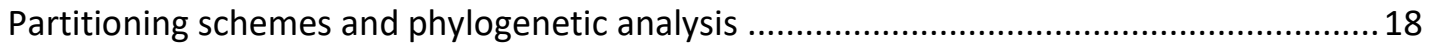

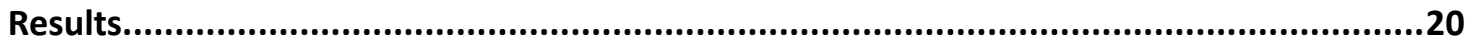

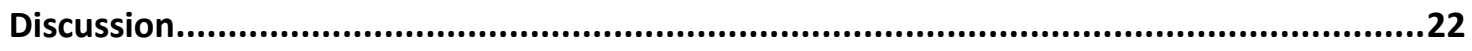

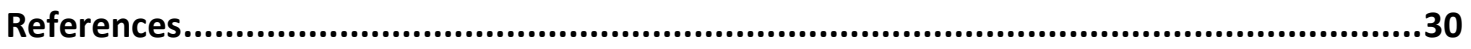

Chapter 2: Anchored enrichment dataset for true flies (order Diptera) reveals insights into the phylogeny of flower flies (family Syrphidae)

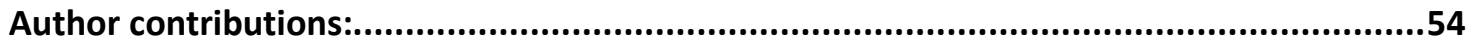

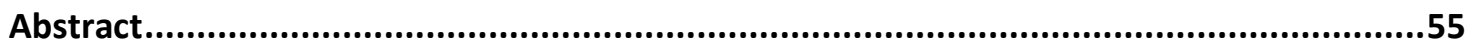

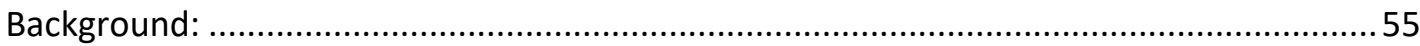

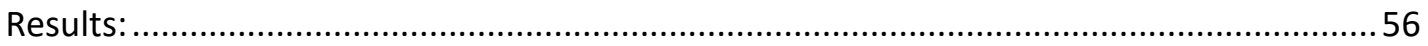

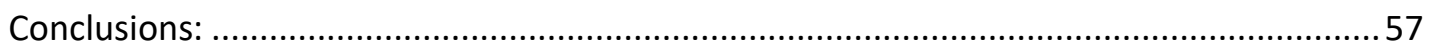

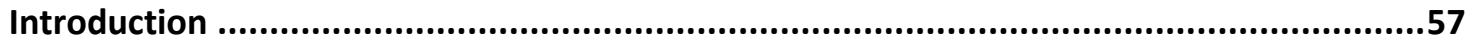

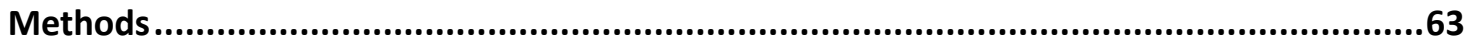

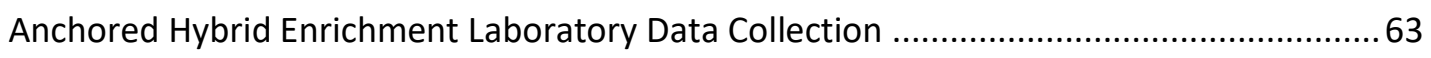

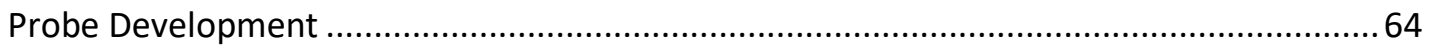

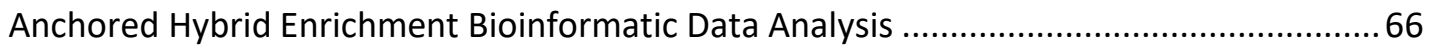

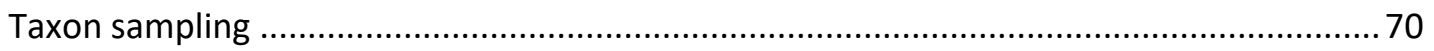

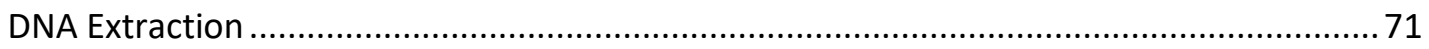

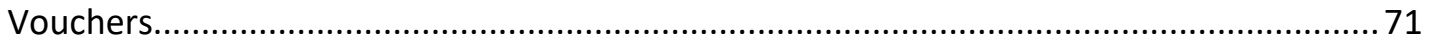

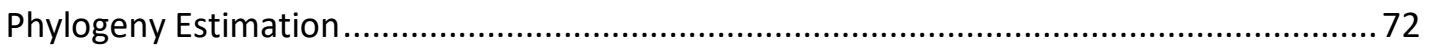




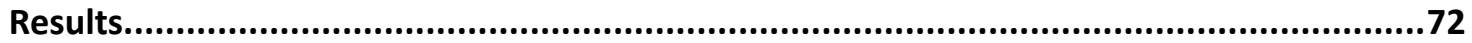

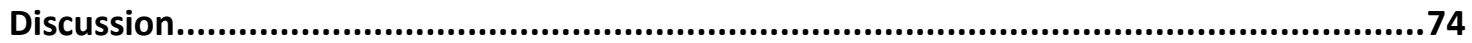

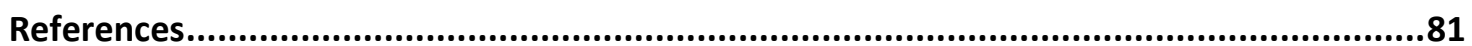

Chapter 3: Revision of Australian Psilota Meigen, 1822 (Diptera: Syrphidae), including

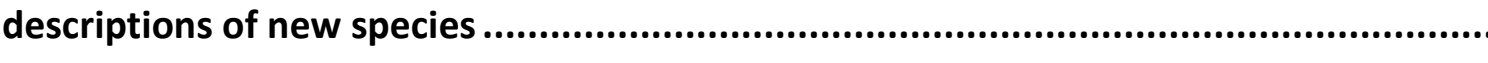

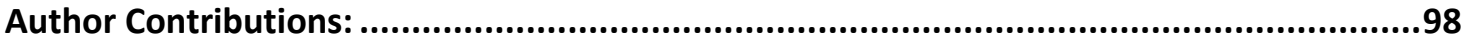

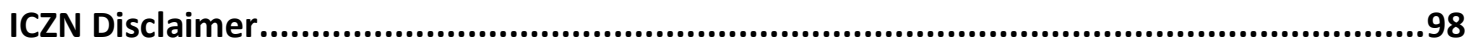

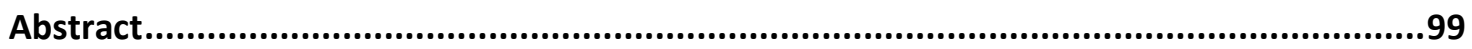

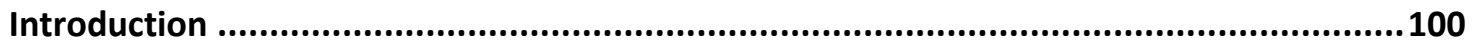

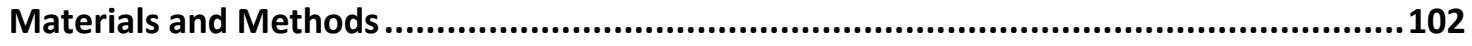

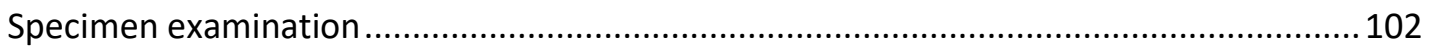

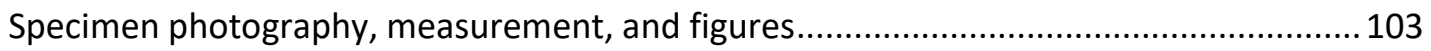

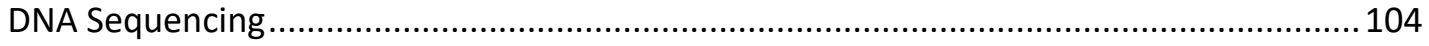

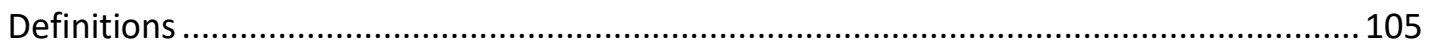

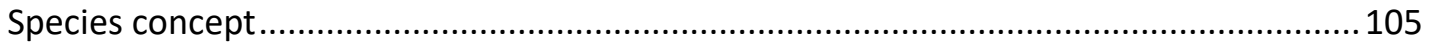

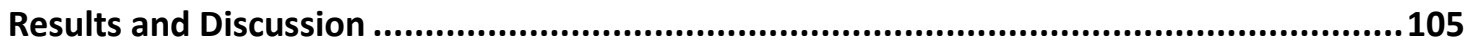

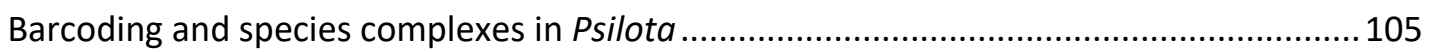

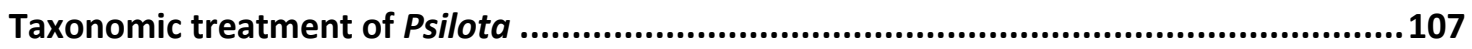

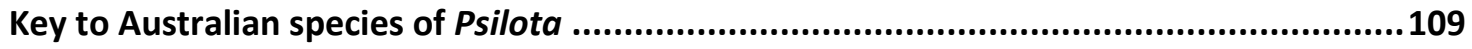

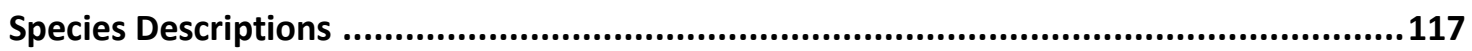

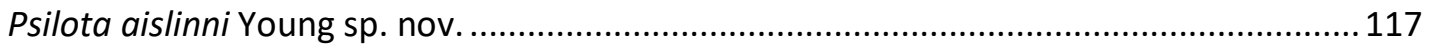

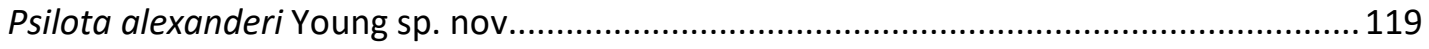

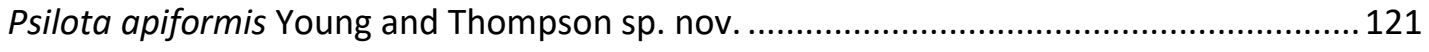

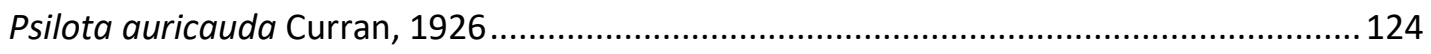

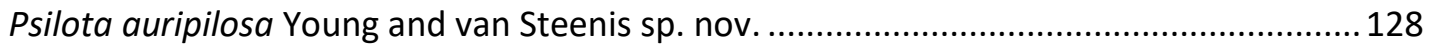

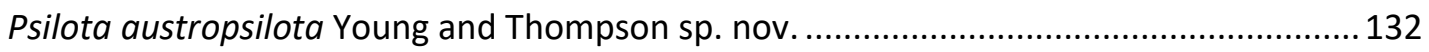

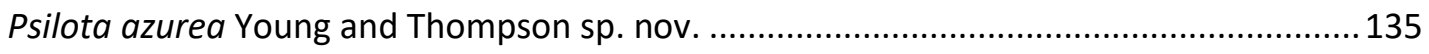

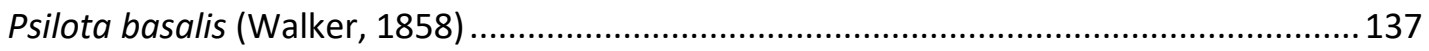

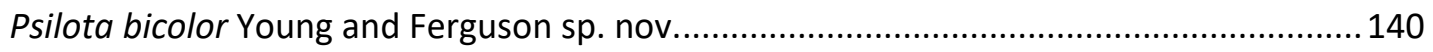

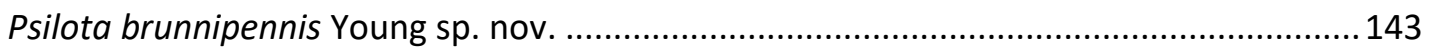

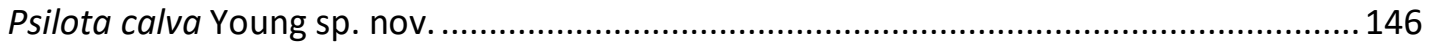

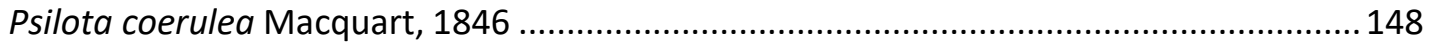

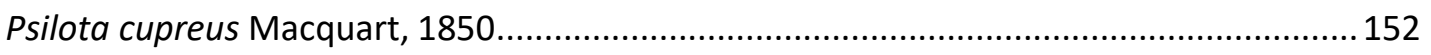

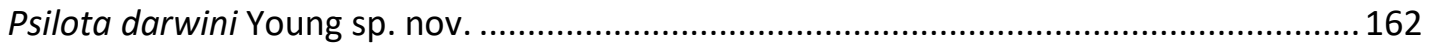

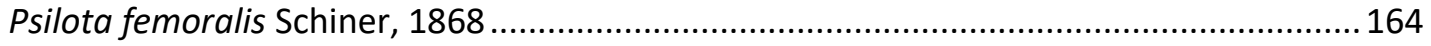

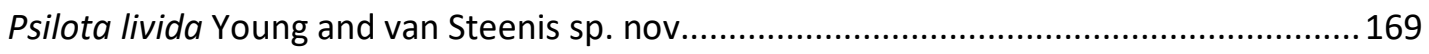

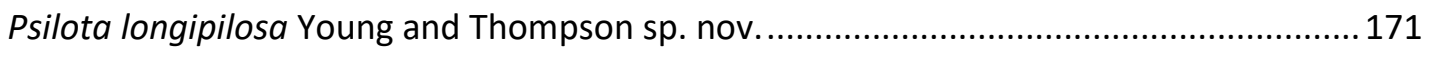

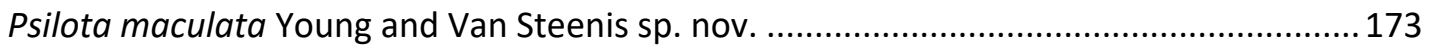

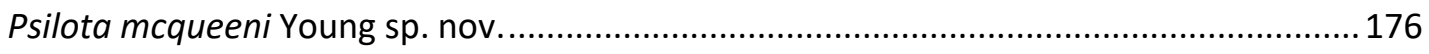

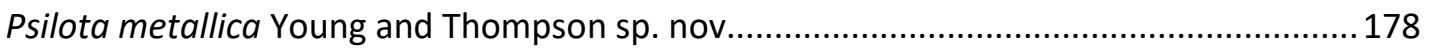

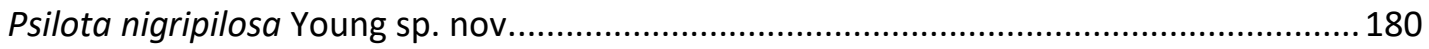

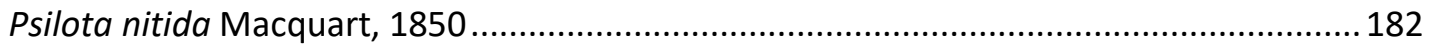

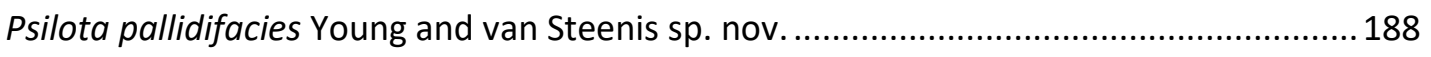

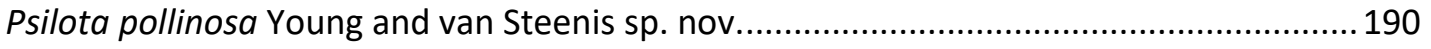




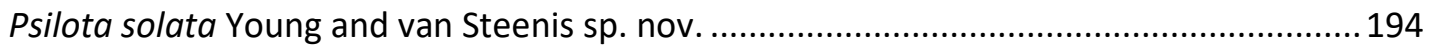

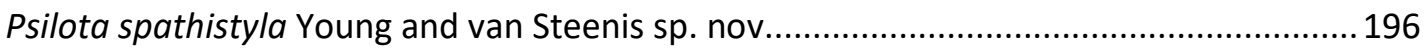

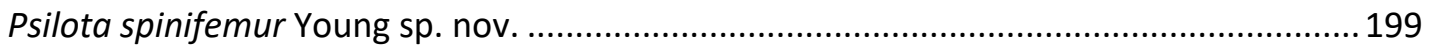

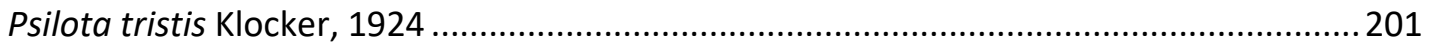

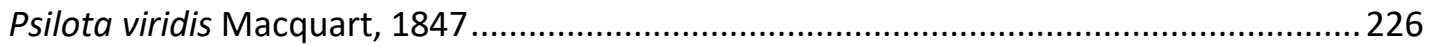

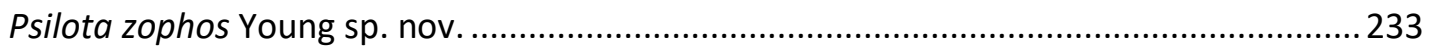

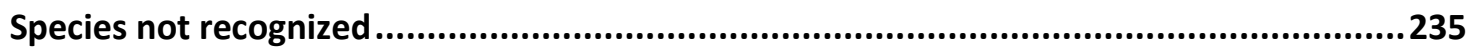

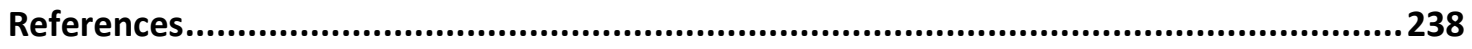

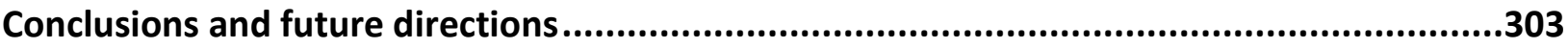




\section{List of Tables}

Chapter 1, Table 1: Taxa used for analyses, original sequencing locations (source), NCBI (National Center for Biotechnology Information) IDs, if published, and collection locality when known. FSU = Florida State University, NCSU = North Carolina State University. 38

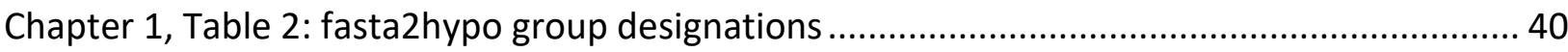

Chapter 1, Table 3: Analyses performed and summary statistics ....................................... 41

Chapter 1, Table 4: Summary of relationships recovered from each analysis ........................ 42

Chapter 2, Table 1: Voucher specimens used to determine exon boundaries for initial probe site selection

Chapter 2, Table 2: Diptera genomes and transcriptomes used to develop probe kit 95

Chapter 2, Table 3: Voucher specimens used in phylogenetic analysis. JSS = Jeff Skevington Specimen. All vouchers deposited in CNC 96

Chapter 3, Table 1: World Psilota names in literature record. Species marked with a * are junior synonyms, species marked with ** have been assigned to different genera, and specimens marked with $\mathrm{a}^{\dagger}$ are junior homonyms. $\mathrm{IM}=$ Indomalayan, $\mathrm{NE}=$ Nearctic, $\mathrm{AU}=$ Australian, $\mathrm{PA}=$ Palaearctic 


\section{List of Illustrations}

Chapter 1, Figure 1. Alternate phylogenetic hypotheses recovered with morphological character data by different authors. A) McAlpine (1989), supported by Brown (1992, 1995). B) Cumming et al. (1995). C) Disney (1994). D) Zatwarnicki (1996). E) Griffiths (1972).

Chapter 1, Figure 2. Phylogenetic hypotheses recovered with molecular data by different authors. A) Collins and Wiegmann (2002) using 28S rDNA. B) Moulton and Wiegmann (2003) using CAD. C) Wiegmann et al. (2011) using 14 nuclear loci, the mitochondrial genome, and 371 morphological characters. D) Pauli et al. (2018) using transcriptomic data.

Chapter 1, Figure 3. Maximum likelihood phylogeny recovered through analysis of the full nucleotide dataset, using IQ-TREE. Partitioning was by-gene, with the most appropriate model of evolution for each gene calculated using IQ-TREE's ModelFinder. 1000 Ultrafast Bootstraps were performed, with results printed to the right of each node. 45

Chapter 1, Figure 4. Maximum likelihood phylogeny recovered through analysis of AHE nucleotide dataset, using IQ-TREE. Metapartitions and model selection were calculated using IQ-TREE's Modelfinder. 1000 Ultrafast Bootstraps were performed, with results printed to the right of each node.

Chapter 1, Figure 5. Maximum likelihood phylogeny recovered through analysis of the full amino acid dataset, using IQ-TREE. Partitioning was by-gene, with the most appropriate model of evolution for each gene calculated using IQ-TREE's ModelFinder. 1000 Ultrafast Bootstraps were performed, with results printed to the right of each node.

Chapter 1, Figure 6. Maximum likelihood phylogeny recovered through analysis of AHE amino acid dataset, using IQ-TREE. Metapartitions and model selection were calculated using IQ-TREE's Modelfinder. 1000 Ultrafast Bootstraps were performed, with results printed to the right of each node.

Chapter 1, Figure 7. Maximum likelihood phylogeny recovered through analysis of full nucleotide dataset, using RAxML. Partitioning was by-gene, using the GTR model of evolution. 100 bootstraps were performed, with results printed to the right of each node.

Chapter 1, Figure 8. Maximum likelihood phylogeny recovered through analysis of AHE nucleotide dataset, using RAxML. Metapartitions were calculated using PartitionFinder2, and the GTR model of evolution was used. 100 bootstraps were performed, with results printed to the right of each node. 
Chapter 1, Figure 9. Coalescent modeling phylogeny recovered through analysis of AHE nucleotide dataset, using ASTRAL-II. Individual gene trees were calculated in RAxML as input for ASTRAL-II.

Chapter 1, Figure 10. Bayesian phylogeny recovered through analysis of AHE nucleotide dataset, using MrBayes 3.2.6. The analysis was run for 10 million generations. Posterior probabilities are printed to the right of each node.

Chapter 1, Figure 11. Bayesian phylogeny recovered through analysis of AHE amino acid dataset, using MrBayes 3.2.6. The analysis was run for 5 million generations. Posterior probabilities are printed to the right of each node. .....

Chapter 2, Figure 1: The ML phylogenetic tree based on the sequenced taxa using RAxML under the model GTR+G. Bootstrap support values are depicted above the nodes. Legend: black: outgroups; green: Microdontinae; orange: Eristalinae; red: Pipizinae; and blue: Syrphinae. Figure by Ximo Mengual.

Chapter 3, Figure 1. Psilota alexanderi, general habitus. Illustration by Jessica Hsiung. 244 Chapter 3, Figure 2. Psilota alexanderi, dorsal habitus. Illustration by Jessica Hsiung. 245

Chapter 3, Figure 3. Psilota alexanderi, lateral habitus. Illustration by Jessica Hsiung............. 246 Chapter 3, Figure 4. Psilota alexanderi, genitalia lateral. Illustration by Jessica Hsiung. 247

Chapter 3, Figure 5. P. aislinni. A) male dorsal habitus, B) male anterolateral face, C) male lateral habitus.

Chapter 3, Figure 6. P. alexanderi. A) male dorsal habitus, B) male anterolateral face, C) male lateral habitus.

Chapter 3, Figure 7. P. apiformis. A) male dorsal habitus, B) male anterolateral face, C) male lateral habitus.

Chapter 3, Figure 8. P. auricauda. A) male dorsal habitus, B) male anterolateral face, C) male lateral habitus.

Chapter 3, Figure 9. P. auricauda. A) female dorsal habitus, B) female anterolateral face, C) female lateral habitus.

Chapter 3, Figure 10. P. auripilosa. A) male dorsal habitus, B) male anterolateral face, C) male lateral habitus.

Chapter 3, Figure 11. P. austropsilota. A) male dorsal habitus, B) male anterolateral face, C) male lateral habitus.

Chapter 3, Figure 12. P. azurea. A) male dorsal habitus, B) male anterolateral face, C) male lateral habitus. 
Chapter 3, Figure 13. P. basalis. A) male dorsal habitus, B) male anterolateral face, C) male lateral habitus.

Chapter 3, Figure 14. P. bicolor. A) male dorsal habitus, B) male anterolateral face, C) male lateral habitus.

Chapter 3, Figure 15. P. brunnipennis. A) male dorsal habitus, B) male anterolateral face, C) male lateral habitus.

Chapter 3, Figure 16. P. calva. A) male dorsal habitus, B) male anterolateral face, C) male lateral habitus.

Chapter 3, Figure 17. P. coerulea. A) male dorsal habitus, B) male anterolateral face, C) male lateral habitus.

Chapter 3, Figure 18. P. cupreus. A) male dorsal habitus, B) male anterolateral face, C) male lateral habitus.

Chapter 3, Figure 19. P. darwini. A) male dorsal habitus, B) male anterolateral face, C) male lateral habitus.

Chapter 3, Figure 20. P. femoralis. A) male dorsal habitus, B) male anterolateral face, C) male lateral habitus.

Chapter 3, Figure 21. P. lividus. A) male dorsal habitus, B) male anterolateral face, C) male lateral habitus.

Chapter 3, Figure 22. P. longipilosa. A) male dorsal habitus, B) male anterolateral face, C) male lateral habitus.

Chapter 3, Figure 23. P. maculata. A) male dorsal habitus, B) male anterolateral face, C) male lateral habitus.

Chapter 3, Figure 24. P. mcqueeni. A) male dorsal habitus, B) male anterolateral face, C) male lateral habitus.

Chapter 3, Figure 25. P. metallica. A) male dorsal habitus, B) male anterolateral face, C) male lateral habitus.

Chapter 3, Figure 26. P. nigripilosa. A) male dorsal habitus, B) male anterolateral face, C) male lateral habitus.

Chapter 3, Figure 27. P. nitida. A) male dorsal habitus, B) male anterolateral face, C)

male lateral habitus.

Chapter 3, Figure 28. P. pallidifacies. A) male dorsal habitus, B) male anterolateral face,

C) male lateral habitus.

Chapter 3, Figure 29. P. pollinosa. A) male dorsal habitus, B) male anterolateral face, C) male lateral habitus. 
Chapter 3, Figure 30. P. solata. A) male dorsal habitus, B) male anterolateral face, C) male lateral habitus.

Chapter 3, Figure 31. P. spathistyla. A) male dorsal habitus, B) male anterolateral face, C) male lateral habitus.

Chapter 3, Figure 32. P. spinifemur. A) male dorsal habitus, B) male anterolateral face, C) male lateral habitus.

Chapter 3, Figure 33. P. tristis. A) male dorsal habitus, B) male anterolateral face, C) male lateral habitus.

Chapter 3, Figure 34. P. viridis. A) male dorsal habitus, B) male anterolateral face, C) male lateral habitus.

Chapter 3, Figure 35. P. zophos. A) male dorsal habitus, B) male anterolateral face, C) male lateral habitus.

Chapter 3, Figure 36. Lateral and dorsal male genitalia of A, B) P. aislinni; C, D) $P$. alexanderi; E, F) P. apiformis, and G, H) P. auricauda.

Chapter 3, Figure 37. Lateral and dorsal male genitalia of A, B) P. auripilosa; C, D) $P$. austropsilota; E, F) P. azurea, and G, H) P. basalis.

Chapter 3, Figure 38. Lateral and dorsal male genitalia of A, B) P. bicolor; C, D) $P$. brunnipennis; E, F) P. calva, and G, H) P. coerulea.

Chapter 3, Figure 39. Lateral and dorsal male genitalia of A, B) $P$. darwini; C, D) $P$. femoralis; E, F) P. lividus, and G, H) P. longipilosa.

Chapter 3, Figure 40. Lateral and dorsal male genitalia of A, B) P. maculata; C, D) P. mcqueeni; E, F) P. metallica, and G, H) P. nigripilosa.

Chapter 3, Figure 41. Lateral and dorsal male genitalia of A, B) P. nitida; C, D) P. pallidifacies; E, F) P. pollinosa, and G, H) P. solata.

Chapter 3, Figure 42. Lateral and dorsal male genitalia of A, B) P. spathistyla; C, D) $P$. spinifemur; E, F) P. viridis; and G, H) P. zophos

Chapter 3, Figure 43. Neighbor-joining tree of all available Australian Psilota DNA

Barcodes. 286

Chapter 3, Map 1: Psilota aislinni 288

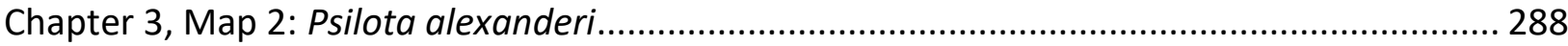

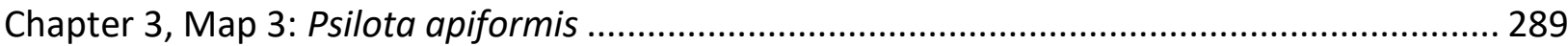

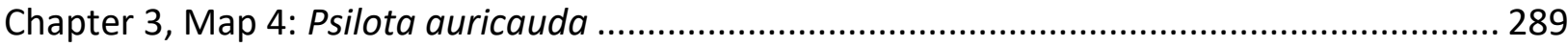

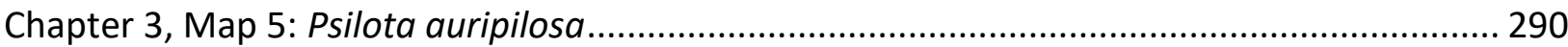




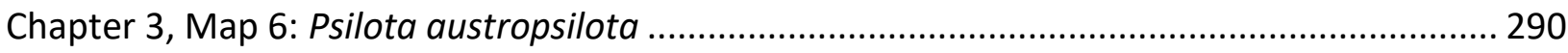

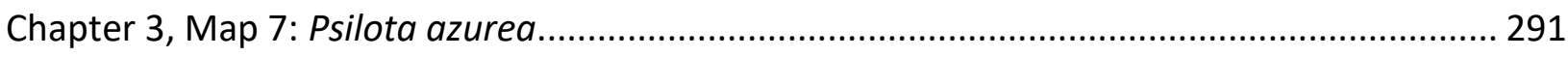

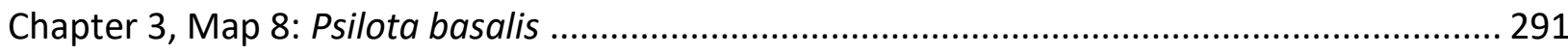

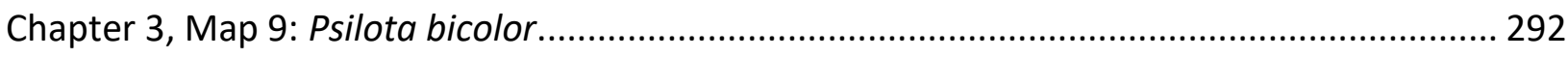

Chapter 3, Map 10: Psilota brunnipennis ................................................................................ 292

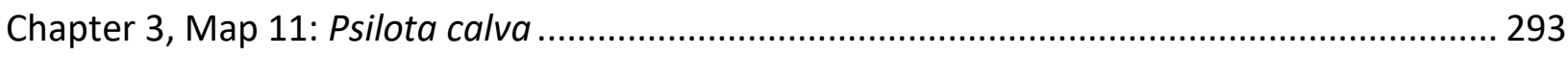

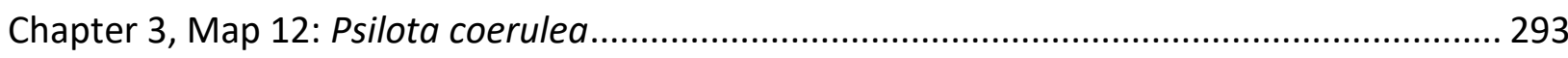

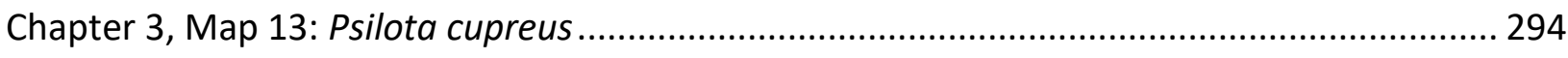

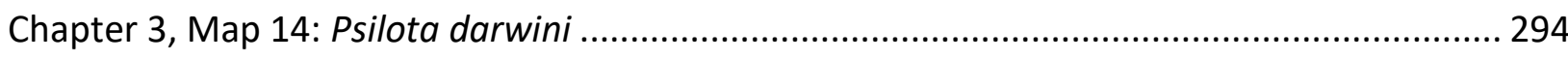

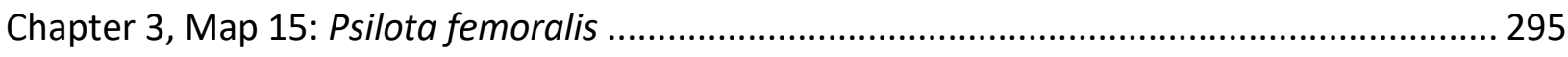

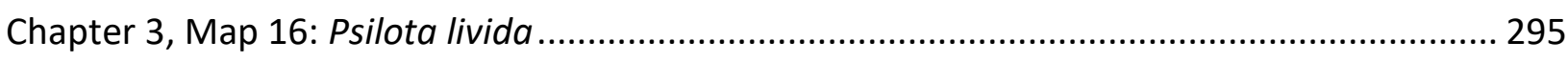

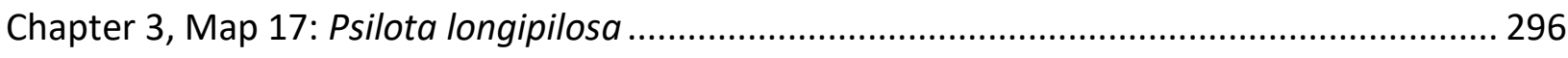

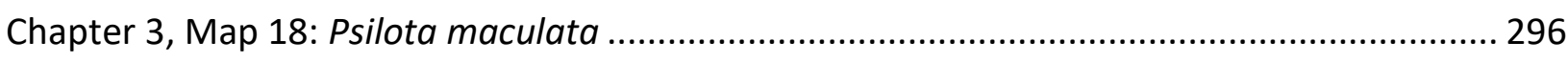

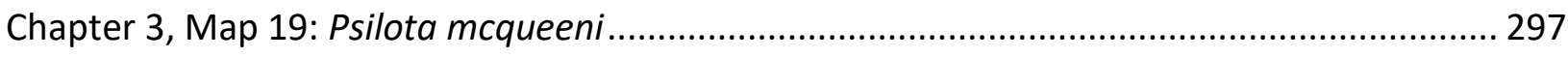

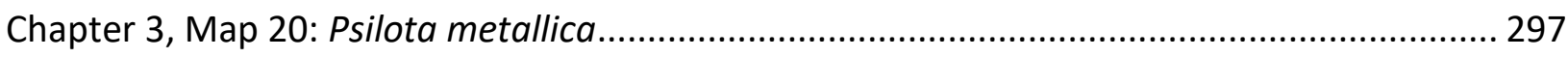

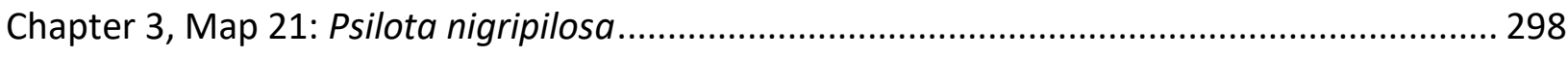

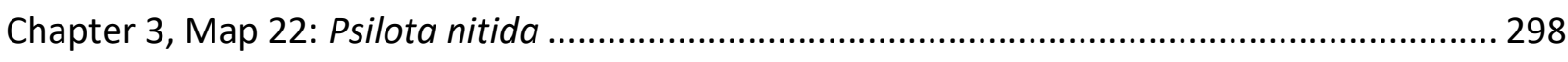

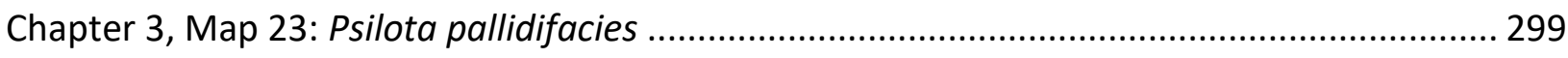

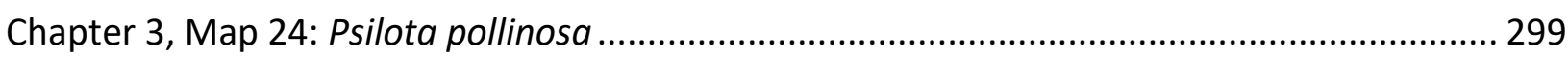

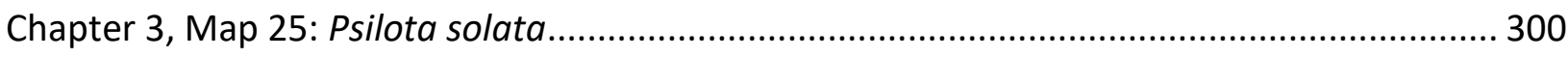

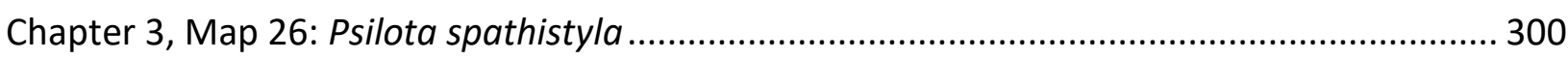

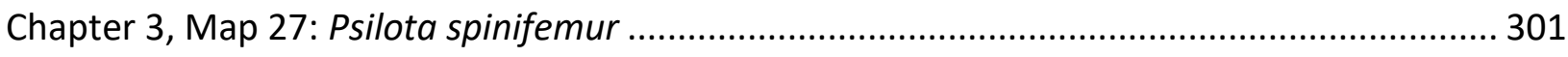

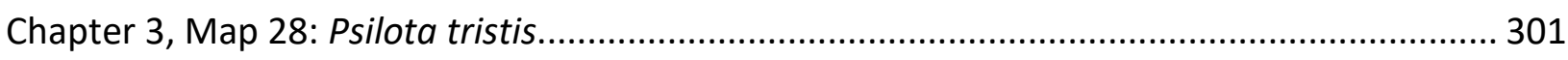

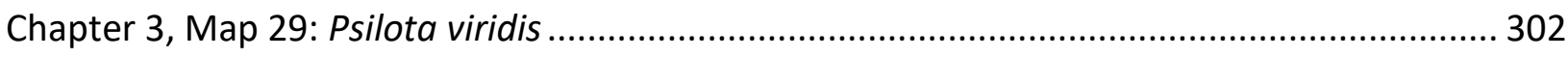

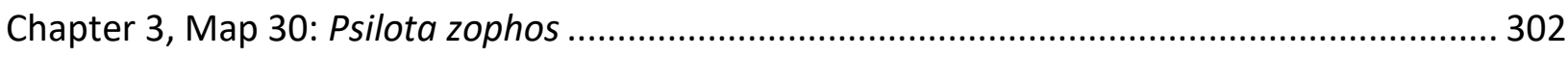




\section{General Introduction}

Numbering over 162,000 described species (Pape and Thompson 2013), Dipterans, or "true flies", are one of the world's most diverse groups of animals. Owing to their role in the spread of malaria and other zoonotic diseases (a mere few dozen species are responsible for millions of deaths per year) (Coatze 2017), and their significant economic impact as agricultural pests (Harris 2017), flies are responsible for great and measurable impact upon humanity. This influence is not solely detrimental, however, since the majority of flies are beneficial insects providing a variety of ecological services. Adults of countless species are regular flower visitors, and we are just beginning to appreciate their importance as both wild and agricultural pollinators (Rader et al. 2016). Many fly larvae feed in decaying organic matter, helping to recycle nutrients by processing and decomposing manure and carrion (Skevington and Dang 2002, Marshall and KirkSpriggs 2017). Some flies are also species-specific parasitoids - having adopted a highly-specialized lifestyle in which larva develop inside another live invertebrate (Marshall and Kirk-Spriggs, 2017). This habit can be readily employed in biological pest control programs (Harris 2017). Despite their incredible influence on our world, we know little about the life history of most described Diptera. This gap in knowledge obscures the true scope of the Dipteran impact on both human and environmental health.

Adult Diptera are characterized by one pair of functional wings, hind wings modified into flight-stabilization organs called halteres, and mouthparts in the form 
of a proboscis, where the labrum, mandibles, hypopharynx, and maxillae, when present, are enclosed together in the trough-like labium (Cumming and Wood, 2017). Taxonomically, the order is divided into the lower Diptera ("Nematocera") and the Brachycera (Wiegmann and Yeates 2017). The lower Diptera are a paraphyletic assemblage, characterized in the past by the plesiomorphic long, filamentous antennae. Familiar lower Diptera include mosquitoes, crane flies, midges, and black flies. Conversely, the Brachycera represent a clade, recognized by the synapomorphic short antennae with eight or fewer flagellomeres. Familiar Brachyceran Diptera include the house flies (Muscidae), horse flies (Tabanidae), flower flies (Syrphidae), and fruit flies (Tephritidae - Drosophilidae, although often called "fruit flies" are more properly referred to as "vinegar flies"). Akin to the division between lower Diptera and Brachycera, the Brachyceran Diptera are subdivided into a paraphyletic lower Brachycera, and a monophyletic Cyclorrhapha, characterized by the pupation inside the modified last larval exuvia, known as a puparium (Skevington and Dang 2002, Wiegmann and Yeates 2017). The Cyclorrhaphans can be further divided into a paraphyletic lower Cyclorrhapha, and a monophyletic Schizophora, characterized by a modified inflatable head segment, which is pumped full of air at the time of emergence and used to knock the "cap" off of the puparium. After emerging, this inflatable sac retracts into the head and is sealed shut, resulting in a diagnostically useful scar (Skevington and Dang 2002, Wiegmann and Yeates 2017).

The phylogenetic relationships of the families comprising the lower Cyclorrhapha (Opetiidae, Phoridae, Platypezidae, Ironomyiidae, Lonchopteridae, 
Syrphidae, and Pipunculidae) are the focus of this thesis' first chapter. The phylogenetic placement of these seven families is notoriously contentious: multiple authors have proposed differing morphological homology statements and phylogenetic hypotheses. Recent molecular phylogenies, which tend to suggest different placements of several key families than morphological analyses, further complicate the matter. Owing to its historic and contemporary phylogenetic uncertainty, this section of the tree of Dipteran life offers rich territory to be explored by specialists working on the order.

In an attempt to stabilize the placement of lower Cyclorrhaphan families, we produced a robust phylogeny employing a combination of anchored hybrid enrichment (AHE) and transcriptomic data for the first chapter of this thesis. Using 43 ingroup taxa and 2446 loci, we produced a phylogeny that is largely concordant with previously published molecular phylogenetic studies, demonstrating the utility of combined transcriptomic and hybrid enrichment data. Opetiidae was recovered as sister to the remainder of Cyclorrhapha, but within this clade excluding Opetiidae, we were unable to unambiguously recover a sister to the remainder of the included families.

The second chapter employs the abovementioned AHE techniques to examine phylogenetic relationships within the family Syrphidae specifically. Syrphidae is a large and fascinating family of lower Cyclorrhapha, exhibiting extremely diverse larval lifestyles and adults that are likely the most significant group of non-bee pollinators worldwide (Rader et al. 2016). Currently, Syrphidae is 
divided into four subfamilies: the Microdontinae, Syrphidae, Pipizinae, and Eristalinae. The former three subfamilies have been recovered as monophyletic in both morphological and molecular analyses while Eristalinae is a longacknowledged paraphyletic taxon. Likewise, tribal classification within Syrphidae is poor, as most eristaline tribes are recovered as non-monophyletic in quantitative analyses. In order to construct a robust phylogeny of Syphidae, we sequenced 599 loci from 30 syrphid species spanning all four subfamilies and 11 out of 15 tribes. As expected, we recovered a paraphyletic Eristalinae alongside a monophyletic Microdontinae, Syrphinae, and Pipizinae. These results mirrored previous molecular phylogenies, enhanced by stronger support values and a more robust dataset.

The final chapter of this thesis is a taxonomic revision of the Australian members of the syrphid genus Psilota Meigen, 1820. Psilota is extremely rare and species-poor throughout the Nearctic, Palaearctic, and Indomalayan region, but common and relatively speciose in the Australian region. Known larvae are found in rotting plant material, usually in decaying sap of wounded trees. Psilota have often been found alongside termites and other wood-boring insects, but this relationship is not well documented beyond a handful of published notes. Despite their relative abundance, the majority of Australian Psilota species remain undescribed. Using a combination of Cytochrome $c$ Oxidase I (COI) DNA Barcoding and traditional morphological examination, we have begun revising the Australian fauna. Twentytwo new species are described, with habitus and genitalia photographs, along with range maps for every species. 
By taking a reductive approach to this project, we have produced phylogenomic hypotheses at a variety of taxonomic depths, in addition to expanding our knowledge of Syrphidae diversity through taxonomic revision. Broad taxonomic works like generic revisions should be placed within a phylogenetic context whenever possible. By first examining the phylogenetic placement of Syrphidae in relation to related families in chapter 1 , and placement of subfamilies and tribes within the family in chapter 2 , the taxonomic revision in chapter 3 is given additional context, as the morphology and known natural history of the group can be compared and contrasted to other Eristalines. Diverse techniques are deployed across all three chapters, all representing taxonomic and phylogenetic techniques that any working systematist should seek to acquire and refine.

When families, subfamilies, tribes and genera are organized in a phylogenetic context, their names become cladistically informative. Our top-down approach helps to ensure that as species are described within a genus, atheir evolutionary relatedness is ensured. Although Psilota was not specifically incorporated in our phylogenetic analyses, our approach offers a compelling tool with which to cast light on life's diversity and relationships at a variety of taxonomic scales.

\section{References}

Coatzee, M. (2017). Medical significance of Diptera. In Kirk-Spriggs, A. H. and $\underline{\text { Sinclair, B. J., eds, Manual of Afrotropical Diptera. Volume 1. Introductory }}$ 
chapters and keys to Diptera families. Suricata 4. South African National Biodiversity Institute, Pretoria.

Cumming, J. M. and D. M. Wood (2017). Adult morphology and terminology. In KirkSpriggs, A. H. and Sinclair, B. I., eds, Manual of Afrotropical Diptera. Volume 1. Introductory chapters and keys to Diptera families. Suricata 4. South African National Biodiversity Institute, Pretoria.

Harris, K. (2017). Agricultural and veterinary significance of Diptera. In KirkSpriggs, A. H. and Sinclair, B. I., eds, Manual of Afrotropical Diptera. Volume 1. Introductory chapters and keys to Diptera families. Suricata 4. South African National Biodiversity Institute, Pretoria.

Marshall, S. A., and Kirk-Spriggs, A. H. (2017). Natural History of Diptera. In KirkSpriggs, A. H. and Sinclair, B. J., eds, Manual of Afrotropical Diptera. Volume 1. Introductory chapters and keys to Diptera families. Suricata 4. South African National Biodiversity Institute, Pretoria.

Pape, T. and F. C. Thompson. (last updated 13 June 2013). "Systema Dipterorum. The Biosystematic Database of World Diptera. Version 1.5." 1.5. Retrieved 5 March 2018, from http://www.diptera.org/.

Rader, R., I. Bartomeus, L. A. Garibaldi, M. P. Garratt, B. G. Howlett, R. Winfree, S. A. Cunningham, M. M. Mayfield, A. D. Arthur and G. K. Andersson (2016). "Nonbee insects are important contributors to global crop pollination." Proc Natl Acad Sci 113(1): 146-151.

Skevington, Jeffrey H., and P. T. Dang. (2002). "Exploring the diversity of flies (Diptera)." Biodiversity. 3(4): 3-27. 
Wiegmann, B. M. and Yeates, D. K. (2017). Phylogeny of Diptera. In Kirk-Spriggs, A. H. and Sinclair, B. J., eds, Manual of Afrotropical Diptera. Volume 1. Introductory chapters and keys to Diptera families. Suricata 4. South African National Biodiversity Institute, Pretoria. 


\section{Chapter 1: Phylogenetics of lower Cyclorrhapha using transcriptome and hybrid enrichment data}

A. D. Young, K. M. Bayless, J. H. Skevington, S. Kelso, B. Misof, B. M. Wiegmann

\section{Author Contributions}

A. D. Young: Sequence alignment, phylogenetic analyses, wrote all text and produced all figures

K. M. Bayless: Mentorship and tutoring of A.D. Young during sequence alignment, provided NCSU sequences

J. H. Skevington: Project conception and design, manuscript editing and revision, specimen collection

S. Kelso: DNA extraction, sequencing, protocol vetting

B. Misof: Initial analysis, pipeline design, 1KITE coordinator

B. M. Weigmann: Mentorship and tutoring of A. D. Young during phylogenetic analyses, 1KITE liaison, provided NCSU sequences 


\section{Abstract}

A phylogenetic hypothesis for lower Cyclorrhapha (Diptera: Brachycera) is constructed using existing transcriptome and hybrid enrichment sequence data. A summary of existing phylogenies of the group is provided, as well as a discussion of the morphological character homologies that have been suggested based on these phylogenetic hypotheses. The present analysis is performed using 43 ingroup taxa and a variety of analytical parameters and programs. Our final results are largely concordant with previously published molecular phylogenetic studies, and demonstrate the utility of combined transcriptomic and hybrid enrichment data. Major phylogenetic relationships recovered include: Apystomyiidae sister to Cyclorrhapha, Lonchopteridae as sister to either the remainder of Cyclorrhapha (in nucleotide analyses) or sister to the remainder of Platypezoidea (in amino acid analyses), Opetiidae sister to Microsania Zetterstedt, either as part of Platypezidae or separate from it, and Pipunculidae sister to Schizophora. Our failure to unambiguously recover a sister family to the remainder of Cyclorrhapha demonstrate that the phylogenetics of this group will remain a contentious issue for the foreseeable future.

\section{Introduction}

Cyclorrhapha is an extremely well-supported clade of Diptera and one of the most speciose radiations of life on Earth, with over 64,000 described species (Wiegmann et al. 2011, Pape and Thompson 2013). There is strong evidence for a 
monophyletic Cyclorrhapha, and the clade can be characterized morphologically by their pupation within the last larval instar (puparium), the lack of adult abdominal plaques, the wing with vein $\mathrm{R}_{4+5}$ unbranched, and other larval synapomorphies (Cumming, Sinclair et al. 1995). The clade has also been recovered in molecular phylogenetic studies using 28S (Collins and Wiegmann 2002) and CAD (Moulton and Wiegmann 2003), as well as a combined morphological and molecular analysis that included 14 nuclear loci, the mitochondrial genome, and 371 morphological characters (Wiegmann et al. 2011). Cyclorrhaphans can be found in nearly any terrestrial microhabitat in every zoogeographic region. Many Cyclorrhaphan families include species with significant human impact, including disease vectors like house flies (Muscidae) and tsetse flies (Glossinidae), crop pests like true fruit flies (Tephritidae) and vinegar flies (Drosophilidae), and pollinators like flower flies (Syrphidae).

Traditionally, the clade has been divided into the lower Cyclorrhapha ("Aschiza") and Schizophora, with the lower Cyclorrhapha lacking the ptilinum (and resulting ptilinal suture) used to push open the puparium as the adult insect emerges. Because of the incredible success and diversity of the Schizophoran lineage, recovering the sister group to this massive radiation of Diptera is of great significance to anyone studying the evolution of Diptera. Although McAlpine (1989) considered the lower Cyclorrhapha to be a monophyletic group sister to the Schizophora on the basis of fused hypophyrangeal and tentorophyrangeal plates in the larvae, most other authors have recovered the group as paraphyletic, using either morphological (Griffiths 1972, Cumming et al. 1995, Zatwarnicki 1996) or 
molecular (Collins and Wiegmann 2002, Moulton and Wiegmann 2003, Pauli et al. 2018) characters. Authors using morphological data (Griffiths 1972, Cumming et al. 1995, Zatwarnicki 1996) have recovered Syrphoidea (Syrphidae + Pipunculidae) as sister to the Schizophora, while molecular phylogenies recover either Syrphidae as sister to Pipunculidae + Schizophora (Collins and Wiegmann 2002, Wiegmann et al. 2011, Pauli et al. 2018), or Pipunculidae sister to Syrphidae + Schizophora (Mouton and Wiegmann 2003).

The lower Cyclorrhapha is comprised of seven families: Opetiidae, Lonchopteridae, Platypezidae, Phoridae, Ironomyiidae, Syrphidae, and Pipunculidae. An eighth family, Sciadoceridae, is now generally included within Phoridae (Disney 2001). This reclassification is based on a combination of fossil evidence of intermediate forms between sciadocerids and more typical Phoridae, and a reinterpretation of several characters suggested as autapomorphic for sciadocerids (Disney 2001). Relationships between these families have long been a contentious issue, with differing interpretations of male genitalic homology within Eremoneura (Cyclorrhapha + Empidoidea) leading to divergent phylogenetic hypotheses. Central to this debate is the segmental origin of the dorsal (epandrium) and ventral (hypandrium) sclerites of the male genitalia and of the paired clasping structures (surstyli) that articulate with the dorsal sclerite itself. Four main interpretations exist, which have been referred to as the periandrial, epandrial, revised epandrial, and hinge hypotheses. Briefly, the periandrial hypothesis (Griffiths 1972) states that the hypandrium is derived from a portion of the $9^{\text {th }}$ sternite, the dorsal sclerite represents a neostructure (the periandrium) formed from the mid-dorsal fusion of 
the basal segment (gonocoxite) of the biarticled clasper (gonopod) derived from sternite 9 seen in nematocerous Diptera and lower Brachycera, while the surstyli represent the distal segment (gonostyle) of the basally fused gonopod. The epandrial hypothesis (Crampton 1936, Hennig 1936, 1958, 1976, McAlpine 1981) considers the epandrium to be derived from tergite 9, the hypandrium to be derived from sternite 9 , and the surstylus as a fusion of the $10^{\text {th }}$ sclerites. The revised epandrial hypothesis (Cumming et al. 1995) considers the epandrium to be derived from tergite 9 , the surstyli to be secondary lobes of tergite 9 , and the hypandrium to represent a fusion of the lower Brachyceran hypandrium and gonocoxites (both derived from sternite 9). The hinge hypothesis (Zatwarnicki 1996) considers the surstyli to be derived from the gonostyle of the lower Diptera, and the epandrium and hypandrium to be derived from tergite and sternite 9 respectively. A graphical summary of the phylogenetic hypotheses resulting from these interpretations of genitalic homology is shown in Figure 1, and a detailed review of the history of Dipteran male genitalic homology can be found in Sinclair et al. (2013).

Cyclorrhaphan phylogenetic relationships have also been recovered using molecular evidence. A $2.7 \mathrm{~kb}$ fragment of the $28 \mathrm{~S}$ ribosomal DNA gene was used to construct a phylogeny of the lower Cyclorrhapha (Collins and Wiegmann 2002), as was the majority of the carbomoylphosphate synthase (CPS) domain of CAD (ca. 4kb) (Moulton and Wiegmann 2003). A combined morphological/molecular analysis was also conducted using 14 nuclear loci, the mitochondrial genome, and 371 morphological characters (Wiegmann et al. 2011). Strikingly, none of these molecular analyses recovered the Syrphoidea (Syrphidae + Pipunculidae) as 
monophyletic, and instead recovered Pipunculidae as the sister to Schizophora. Major results of these studies are summarized in Figure 2.

Throughout all of these studies, several contentious issues remain: the placement of the families Opetiidae and Lonchopteridae, the monophyly of the Syrphoidea, and the sister group to the Cyclorrhapha itself. Previous morphological studies have recovered either Opetiidae or Lonchopteridae as sister to the remainder of the Cyclorrhapha, while molecular studies have traditionally recovered two sister clades comprised of Lonchopteridae + Opetiidae + Platypezidae + Phoridae (incl. Sciadocera White, 1916) and Syrphidae + Pipunculidae + Schizophora. Likewise, all morphological studies that have recovered a paraphyletic lower Cyclorrhapha have also recovered a monophyletic Syrphoidea (Syrphidae + Pipunculidae) sister to the Schizophora, whereas previous molecular studies have recovered Syrphidae sister to Pipunculidae + Schizophora. Finally, most morphological studies have recovered the Empidoidea as sister to the Cyclorrhapha, while molecular evidence points to Apystomyiidae as the sister to Cyclorrhapha, with Empidoidea as sister to Apystomyiidae + Cyclorrhapha.

The objective of the present study is to produce a robust phylogeny of the lower Cyclorrhapha. This will be accomplished using a combination of existing transcriptome data produced by the 1000 Insect Transcriptome Evolution (1KITE) initiative [https://www.1kite.org] (Misof et al. 2014), existing anchored hybrid enrichment data taken from Young et al. (2016), and novel anchored hybrid enrichment data extracted following the protocol published in Young et al. (2016). 
Due to their phylogenetic position within Cyclorrhapha in relation to Schizophora, debate over the evolution of male genitalia, lingering questions as to the sister group to the Cyclorrhapha, and the large amount of existing molecular data relevant to this project, lower Cyclorrhaphan families represent an excellent opportunity to test the utility of mixed transcriptome and hybrid enrichment data in the construction of phylogenetic hypotheses. By doing so, we hope to resolve some long-standing issues of family placement, and highlight problem areas that require further investigation.

\section{Materials and Methods}

\section{Taxon sampling and data collection}

Taxon sampling was designed to include exemplars from all major lower Cyclorrhaphan lineages as well as 12 outgroup taxa including highly autapomorphic taxon Apystomyia elinguis Melander (Apystomyiidae). The final dataset includes 43 taxa. Most exemplars were initially sequenced as part of several different projects, including 1KITE and Young et al. (2016), while others were taken from the NCBI Sequence Read Archive (SRA). Many of the key taxa in this analysis were sequenced simultaneously with the exemplars used in Young et al. (2016), but were not included in that analysis. Finally, several additional taxa were sequenced at North Carolina State University using the probe kit and protocol described in Young et al. (2016). See Table 1 for a full list of taxa and their original sequencing locations. 


\section{Sequence filtering and preprocessing}

Aside from transcriptome assemblies provided by 1KITE, all AHE and NCBI SRA data underwent a quality check and trimming step prior to assembly. FastQC Reports (Andrews 2010) and Trimmomatic (Bolger et al. 2014) were used in tandem to achieve this end. Trimmomatic was used on each sequence in turn until FastQC reported a "per base quality" > 20 in all cases, and adapter sequences were removed entirely, as reported in the "Adapter Content" tab of the FastQC output. Trimmomatic was run using the following command: "java -jar ./trimmomatic0.36.jar PE ./I3772_U1.fastq ./I3772_U2.fastq ./I3772_U1_pairedoutput.fastq.gz ./I3772_U1_unpairedoutput.fastq.gz ./I3772_U2_pairedoutput.fastq.gz ./I3772_U2_unpairedoutput.fastq.gz ILLUMINACLIP:/Applications/Trimmomatic0.36/adapters/TruSeq3-PE.fa:2:30:10 LEADING:24 TRAILING:24 SLIDINGWINDOW:4:24 MINLEN:50". In general, one round of Trimmomatic was sufficient for the data to pass a subsequent FastQC check, but in several cases up to three rounds of Trimmomatic were required for the data to pass.

\section{Assembly}

Reads from AHE and NCBI SRA data were assembled into contigs at the Canadian National Collection of Insects using Trinity 2.2 (Grabherr, Haas et al. 2011). Trinity was run using the commands “Trinity --seqType fq --max_memory 50G --left reads_1.fq --right reads_2.fq --SS_lib_type RF --CPU 6". Taxa sampled by 1KITE were assembled with SOAPdenovo-Trans (Xie et al. 2014). 


\section{Orthology Assessment}

Orthograph (Petersen et al. 2017) was used for orthology assessment of all sequences. An Orthograph database was created using the Mecopterida orthology reference set (Meusemann \& Vasilikopoulos, unpublished), which consists of the sequenced and annotated genomes and transcriptomes from five model organisms available on orthodb7 (Kriventseva et al. 2014) (http://cegg.unige.ch/orthodb7): Drosophila melanogaster Meigen, Glossina morsitans Westwood, Aedes aegypti L., Bombyx mori L., and Danaus plexippus L.. Briefly, Orthograph works by creating a database out of the input genomes provided and assigning markers from individual reference taxa to Clusters of Orthologous Genes (COGs), which are aligned singlecopy orthologs present in all reference taxa. These COGs are then populated with profile hidden Markov Models (pHMMs), which are used to search through the target taxon library for candidate orthologs. These candidate orthologous genes are then used in a reciprocal BLAST search using the reference taxa as a database to assign these candidate orthologous genes to COGs.

In all cases, some non-target DNA sequences successfully passed through Orthology prediction. These were detected using NCBI BLAST checks of all markers post-Ortholog. Any sequence that matched to a non-insect with $\mathrm{e}<0.005$ while not matching any insect with $e<0.005$, as well as any sequence that did not match any taxon in the NCBI BLAST database with $\mathrm{e}<0.005$, was considered a potential contaminant and removed from the experimental dataset. This process was 
expedited using a custom perl script that compared two BLAST outputs for each sequence, one that "Insecta" in the BLAST library, and a second with "Insecta" excluded from the BLAST library during the search. Common contaminants included endosymbiotic bacteria such as Rickettsia da Rocha-Lima and Wolbachia Hertig \& Burt, as well as human, arachnid (dust mites), and plant DNA.

\section{Alignment and data subdivisions}

AHE, NCBI SRA, and transcriptome data were prepared and aligned together using a bioinformatic pipeline (Simon et al. in prep.) that has been modified from Misof et al. (2014). Stop codons are masked prior to individual gene alignment with MAFFT (Katoh and Standley 2013), using the L-INS-i alignment method. Outliers are then checked for and removed by comparing the BLOSUM62 substitution rates between all taxa and loci. Realignment of these putative outliers is attempted before final outlier removal. Nucleotide data is aligned to the amino acid data using PAL2NAL (Suyama et al. 2006) in order to inform codon positions of the nucleotide dataset. Aliscore and Alicut (Kück et al. 2010) are used sequentially to identify and remove randomly aligned and uninformative sites within each locus at the amino acid level. At this stage, 308 genes shared between the anchored hybrid enrichment dataset and the transcriptome alignments were concatenated into corresponding amino acid and nucleotide supermatrices for analysis, and is hereafter referred to as the "AHE datasets". The complete dataset underwent further processing, detailed below. 


\section{Full dataset post-processing}

At this stage, the full dataset consisted of 3144 individually aligned loci. MARE (Meyer 2011) was used in conjunction with a custom perl script (Simon et al. in prep.) to detect and remove five loci with an information content of zero. Next, fasta2hypo (Simon et al. in prep.) was used to further reduce missing data. Fasta2hypo is a perl script that takes a list of user-defined taxon groups (given in Table 2) as an input, and then checks for those groups, removing any loci that do not contain sequence data for at least one taxon from each group. This is done to reduce taxon bias in missing data, and further reduced the dataset from 3139 to 2446 loci. These 2446 loci were then concatenated into nucleotide and amino acid supermatrices, and are hereafter referred to as the "full datasets".

\section{Partitioning schemes and phylogenetic analysis}

All datasets (nucleotide and amino acid for both AHE and full datasets) were initially partitioned by locus. For the AHE nucleotide dataset, PartitionFinder2 (Lanfear et al. 2016) and IQ-TREE's Modelfinder (Kalyaanamoorthy et al. 2017) were used independently to create metapartitions from the initial by-locus partitions. Partitionfinder2 was run following settings found in Winterton et al. (Winterton et al. 2017). For the AHE amino acid dataset, PartitionFinder2 failed with an unknown error so only IQ-TREE Modelfinder metapartitions were used for analyses. This was not performed for the full datasets due to computational time 
constraints (estimated time to perform a modeltest in IQ-TREE with the full dataset amino acid alignment was 8500 hours).

Maximum likelihood phylogenetic analyses were performed using RAxML for the nucleotide alignments of both the AHE and full dataset. Metapartitions from both the PartitionFinder2 and IQ-TREE Modeltest outputs were used for the AHE dataset, while the full dataset was partitioned by locus. These analyses were performed on the CIPRES computer cluster with the " $\mathrm{f}-\mathrm{a}$ " option and 100 bootstraps. Analysis of the amino acid alignments in RAxML (Stamatakis 2014) lead to arithmetic errors that were likely a limitation of the current version of the software (Stamatakis, pers. comm.).

Bayesian analyses were performed using MrBayes 3.2.6 (Ronquist et al. 2012) for the nucleotide and amino acid AHE datasets. Metapartitions from the PartitionFinder2 outputs were used for the nucleotide dataset while metapartitions from IQ-TREE's Modelfinder were used for the amino acid dataset. These analyses were run on the CIPRES computer cluster for 10 million generations (nucleotide alignment) and 1 million generations (amino acid alignment).

IQ-TREE (Nguyen et al. 2014) was used to perform maximum likelihood analyses of the amino acid AHE and full datasets due to the arithmetic errors that precluded RAxML analysis. IQ-TREE's Modelfinder (Kalyaanamoorthy et al. 2017) function was also used to reanalyze the per-locus partitions for both the AHE nucleotide and amino acid datasets that were used as input to PartitionFinder2 and create its own best-fit metapartitions with model selections. This was done due to 
the fact that unlike PartitionFinder2, IQ-TREE's Modelfinder incorporates free-rate models into its search. Therefore, any significant differences between phylogenies constructed using PartitionFinder2's and IQ-TREE's partitioning schemes may indicate a negative effect of model mis-fit. Phylogenetic analyses were performed in IQ-TREE using CIPRES, with the "-spp" option enabled and 1000 Ultra-fast Bootstraps (Minh et al. 2013).

Finally, coalescent analysis of the nucleotide AHE dataset was performed in ASTRAL-II (Mirarab and Warnow 2015), using individual gene trees for each locus as input. Gene trees were constructed in RAxML prior to ASTRAL-II analysis. ASTRAL-II was used to perform a phylogenetic analysis in order to test for possible effects of incomplete lineage sorting.

\section{Results}

Of the nine analyses performed, the IQ-TREE nucleotide full dataset was chosen as the preferred tree due its limited congruence with existing morphological phylogenies, shown in Fig 3. All analyses recovered the same major clades with high degrees of support, with three important exceptions (placement of Lonchopteridae, placement of Opetiidae + Microsania Zetterstedt, and monophyly of ((Platypezidae + Opetiidae $)+($ Ironomyiidae + Phoridae $)$ ), discussed in more detail below. Bootstrap support for all major clades in the preferred analysis was 100\%. A summary of analyses performed, as well as summary statistics for all analyses is presented in table 3. 
Within the outgroups, a monophyletic Asiloidea and Empidoidea were recovered in all analyses. Within Empidoidea, a monophyletic Empididae (including Oreogeton Schiner, a genus that is considered incertae sedis within the Empidoidea by Sinclair and Cumming (Sinclair and Cumming 2006)) and Dolichopodidae were recovered in all analyses. Likewise, Apystomyia was universally recovered as sister to Cyclorrhapha.

Within Cyclorrhapha, placement of Lonchopteridae differed between nucleotide (Figs. 3, 4, 7, 8, 9, 10) and amino acid (Figs. 5, 6, 11) analyses. In all nucleotide analyses, Lonchoptera Meigen was recovered as sister to all other Cyclorrhapha, whereas in all amino acid analyses Lonchopteridae was placed in a clade consisting of (Lonchopteridae + ((Opetiidae + Microsania $)+($ Platypezidae + (Ironomyiidae + Phoridae))) .

In all analyses except for the ASTRAL-II analysis, Opetiidae was recovered as sister to Microsania. In other nucleotide analyses, Opetiidae + Microsania was recovered as sister to the remaining Platypezidae, whereas in all amino acid analyses Opetiidae + Microsania was recovered sister to the more inclusive (Platypezidae excl. Microsania + (Ironomyiidae + Phoridae)). In the ASTRAL-II analysis (Fig. 9), Opetia was sister to a monophyletic Platypezidae, with Microsania sister to the remainder of Platypezidae.

Full nucleotide dataset analyses recovered a clade consisting of ((Platypezidae + Opetiidae $)+($ Ironomyiidae + Phoridae $))$ sister to (Syrphidae + (Pipunculidae + Schizophora)), whereas AHE nucleotide analyses recovered 
(Phoridae + Ironomyiidae) sister to ((Platypezidae + Opetiidae $)+($ Syrphidae + (Pipunculidae + Schizophora))), i.e. Phoridea was sister to the remainder of Cyclorrhapha excluding Lonchopteridae.

Syrphoidea was recovered as non-monophyletic in all cases, with Syrphidae sister to Pipunculidae + Schizophora. A summary of clades recovered in each analysis are available in Table 4.

\section{Discussion}

This analysis represents the first use of the AHE Diptera probe kit developed in 2016 for higher-level Diptera phylogenetics. While the 2016 study successfully produced a highly-supported family-level phylogenetic tree appropriate for examining subfamilial relationships within Syrphidae, this was our first attempt to study evolutionary relationships between Dipteran families using this technique. Additionally, this is one of the first examples of combining transcriptomic and hybrid enrichment data for phylogenetic analysis, a technique that was also recently utilized for vespid wasp phylogenetics (Bank et al. 2017). Although the preferred dataset contained a high amount of missing data (63.2\%) due to the nature of our combined dataset, we recovered a phylogeny with near-universally high support values.

Our results are largely concordant with the previously published multigene + morphology analysis of Diptera phylogeny (Wiegmann et al. 2011). Similar to their study, Apystomyiidae was recovered as sister to Cyclorrhapha, with Empidoidea 
sister to Apystomyiiidae + Cyclorrhapha. Apystomyia elinguis is the only member of the family, and has been previously placed in Bombyliidae (Hennig 1973, Woodley 1989) and Therevidae (Sinclair et al. 1993). The placement of Apystomyiidae as sister to Cyclorrhapha is contradicted by study of the male genitalia, which places the family as sister to the Eremoneura (Sinclair et al. 2013). This placement is supported by four synapomorphies (epandrium deeply U-shaped; subepandrial sclerite fully developed, sclerotized from base of hypoproct to base of phallus; paired lateral arms of subepandrial sclerite fused to the inner margin of the epandrium and extending to tips of epandrium; surstyli functionally developed but not articulated) and the lack of three Eremoneuran symapomorphies (gonostyli absent; presence of postgonites; phallic plate) (Sinclair et al. 2013). Additionally, Apystomyiidae lacks synapomorphies that would indicate a close relationship with Cyclorrhapha (imaginal development within a puparium; $360^{\circ}$ circumverted male genitalia; gonocoxal apodeme confined to anterior margin of hypandrium lacking narrow process), with the only a possible precursor to the Cyclorrhaphan phallapodeme as potential support of a close relationship (Sinclair et al. 2013).

Within Cyclorrhapha, our data illustrate that determining the sister taxon to the remainder of the clade will remain a contentious issue. Our nucleotide alignment places Lonchopteridae as the sister to the remainder of the Cyclorrhapha in all analyses performed, while our amino acid alignment splits Cyclorrhapha into two sister clades, one of which includes (Lonchoptera + (Opetiidae + Platypezidae) + (Phoridae + Ironomyiidae)) while the other includes (Syrphidae + (Pipunculidae + Schizophora)). Previous morphological phylogenetic analyses by Griffiths (1972) 
and Zatwarnicki (1996) have placed Lonchopteridae as sister to the remainder of Cyclorrhapha, and this placement was also hinted at by Hennig in his study of the male terminalia of Lonchoptera (Hennig 1976). However, Wiegmann et al. (2011) produced a phylogeny very similar to the current amino acid trees. Unfortunately, their analysis included only one platypezid, so it is impossible to compare their phylogeny directly with the present study in regard to Opetiidae + Platypezidae relationships. Similar to our results, Pauli et al. (2018) recovered Lonchopteridae as either sister to the remainder of Cyclorrhapha or as part of a larger clade including Opetiidae, Platypezidae, Phoridae and Ironomyiidae in an analysis of Syrphoidea relationships using transcriptomics. Three possible causes of this discordance suggested by Pauli et al. (2018) are the heterogeneity of the third codon position in the nucleotide alignment, overparameterization of the chosen nucleotide model (unlikely in our case, as we have experimented with different models in IQ-TREE), and underparameterization of the chosen amino acid model (again unlikely for the same reasons).

All analyses except for the ASTRAL-II analysis recovered Opetiidae as sister to Microsania, either as part of a larger clade with the remainder of Platypezidae in the nucleotide analyses, or with those two taxa together as sister to the remainder of Platypezidae + Phoridae + Ironomyiidae in the amino acid analyses, rendering the current concept of Platypezidae including Microsania paraphyletic. Conversely, the ASTRAL-II alignment recovered Opetiidae as sister to Platypezidae including Microsania. Originally described as a platypezid, Opetia nigra (Meigen) was assigned to the subfamily Opetiinae (Kessel and Maggioncalda 1968) in the first modern 
revision of the Platypezidae before being assigned to its own family by Chvála (1981) on the basis of observations made by Griffiths (1972) and Hennig (1976). Until the recent discovery and description of Puehuemyia chandleri Amorim, Silva and Brown 2018, O. nigra was the sole member of its containing family. Although it has never been placed in its own family, Microsania is also a phylogenetically problematic group. This is mainly due to the fact that most noncontroversial synapomorphies for Platypezidae are larval characters, and the larvae of Microsania (and Opetia) are completely unknown, making it difficult to make strong statements of monophyly. Using COI, COII, CytB, 12S, 16S, and 28S, a recent molecular phylogeny of the Platypezidae by Tkoč et al. (2017) recovered the same topology as the present full nucleotide alignment, with Opetiidae + Microsania as sister to the remainder of Platypezidae. While no morphological characters were used in their phylogeny, they proposed four new synapomorphies for Platypezidae exclusive of Microsania and placed Microsania as incerta sedis within Platypezoidea on the basis of their results. However, two of their four synapomorphies are larval characters so cannot be scored for Opetia or Microsania, whose larval forms remain unknown, and one character is not present in Melanderomyia Kessel, another genus of Platypezidae. This leaves only one character, bifurcated setae on the femur and tibia, which can truly be called a new synapomorphy for Platypezidae exclusive of Microsania. For this reason, we have chosen a more conservative approach in labelling our full nucleotide alignment, with a monophyletic Opetiidae + Platypezidae as our preferred tree as opposed to supporting the removal of Microsania from all currently recognized families. In contrast to this, traditional 
morphological evidence for a clade of Cyclorrhapha exclusive of Opetiidae provides support for a placement of Opetiidae as sister to the remainder of Cyclorrhapha (Cumming et al. 1995, Sinclair et al. 2013). The characters supporting Cyclorrhapha exclusive of Opetiidae include one synapomorphy of the male genitalia (phallapodeme present) and three nongenitalic characters (antenna with threearticled arista; acrostichial and dorsocentral setae differentiated; wing with costa distinctly ending at $\mathrm{R}_{4+5}$, or $\mathrm{M}_{1}$, rather than costa circumambient) (Cumming et al. 1995). However, the recently described opetiid P. chandleri breaks one of these three nongenitalic character states, as it has a three-articled arista like other cyclorrhaphans and unlike O. nigra. This suggests that the two-articled arista of $O$. nigra is a secondary loss, rather than a ground-state for the family, weakening the hypothesis that Opetiidae is sister to the remainder of Cyclorrhapha. Amorim et al. (2018) go on to describe several putative synapomorphies of Opetiidae + "Platypezoidea" (Lonchopteridae + Platypezidae + Ironomyiidae + Phoridae), including the placement of the anterior spiracle relative to the anepisternum and the proepimeron, the elongate shape of the anterior basalare, and the segmentation of the female abdomen (segments 1-3 normal, segment 4 longer, segment 5 shorter, and segments 6-7 fused into an ovipositor). Taken together, new morphological evidence from $P$. chandleri supports the inclusion of Opetiidae within the platypezoids and calls morphological evidence gathered from the examination of $O$. nigra alone into question, suggesting that further study of this enigmatic family is in order. 
Phoridae including "Sciadoceridae" sister to Ironomyiidae was universally recovered in all analyses. This sister-group relationship is nearly universally accepted, regardless of morphological character scoring or molecular analysis used to construct a phylogeny, with Disney $(1988,1994)$ offering the only major disagreement. But, Disney (2001) later revised his hypotheses after examining fossil taxa and included sciadocerids within Phoridae as the sister to the remainder of the family.

The arrangement of (Opetiidae + Platypezidae) and (Ironomyiidae + Phoridae) is also ambiguous, as analyses of the full nucleotide dataset places the large clade ((Opetiidae + Platypezidae $)+($ Ironomyiidae + Phoridae $)$ ) sister to (Syrphidae + (Pipunculidae + Schizophora)), whereas analyses of the AHE nucleotide dataset places (Ironomyiidae + Phoridae) sister to the remainder of Cyclorrhapha excluding Lonchopteridae. This ambiguity may be a result of the large amounts of missing data (63.3\%) in the full dataset that result from combining transcriptome and hybrid enrichment data. Unfortunately, this is difficult to test without obtaining more transcriptome data.

Our recovery of a non-monophyletic Syrphoidea, with Syrphidae sister to Pipunculidae+Schizophora, is concordant with most recent molecular phylogenies of the group (Collins and Wiegmann 2002, Moulton and Wiegmann 2007, Wiegmann et al. 2011, Pauli et al. 2018), and in disagreement with virtually all morphological analyses that have so far recovered a sister group relationship between Syrphidae and Pipunculidae (Syrphoidea) (Hennig 1976, McAlpine 1989, Disney 1994), often 
with Syrphoidea sister to Schizophora (Griffiths 1972, Cumming et al. 1995, Zatwarnicki 1996). The main character used in support of Syrphoidea is the hypopygium deflected under the right side of the abdomen (Cumming, Sinclair et al. 1995) and the associated asymmetrical arrangement of abdominal segments six to eight (Griffiths 1972), and the main character used in support of Syrphoidea + Schizophora is the articulated phallapodeme (Sinclair 2013). In a detailed examination of the Cyclorrhaphan thorax, Tachi (Tachi 2014) proposed an articulation between the metepimeron and the abdomen as a possible synapomorphy of Pipunculidae + Schizophora, supporting our present hypothesis and those of recent molecular phylogenies.

The phylogenetic hypothesis recovered in the present analysis uses a combination of hybrid enrichment and transcriptomic data, and provides a robust alternate hypothesis to those constructed using parsimony analysis of male genitalic characters. This represents the largest collection of data ever brought to bear on the phylogenetic origins of the lower Cyclorrhapha. Notably, this strengthens support for Pipunculidae as sister to the Schizophora, a massive radiation of over 80 families of Diptera. Our phylogeny suggests that character reversals have occurred in several enigmatic taxa, especially Apystomyia elinguis (Apystomyiidae) and Opetia nigra (Opetiidae). In order for Apystomyia to be sister to Cyclorrhapha, three characters reversals are necessary: gonostyli regained, postgonites lost, and phallic plate lost. Likewise, for Opetia to be included within Platypezidae, three (or four, if a twoarticled arista is indeed the ground state for Opetiidae, but see discussion of $P$. chandleri above) character reversals are necessary: loss of the phallapodeme, 
acrostichial and dorsocentral setae undifferentiated, and costa circumambient instead of ending at $\mathrm{R}_{4+5}$ or $\mathrm{M}_{1}$. Unfortunately, as the larvae of both of these genera are unknown, it is impossible to score all available morphological character states for them. Finding and describing the larvae of Opetia and Microsania should be the priority for future work on phylogenetics of the lower Cyclorrhapha.

One of the main conclusions that can be drawn from our work is that reconciling the alternate phylogenetic hypotheses produced through analysis of morphological characters and molecular sequence data will not be an easy task. Combined analysis of morphological and molecular data can easily lead to increasingly large genomic datasets swamping any signal provided by morphological characters (Near 2009), while successive weighting schemes of morphological data can be rightly criticized as largely arbitrary (Kluge 1997). Although molecular datasets are many orders of magnitude larger than morphological datasets, some of the reversals in morphological character states necessitated by accepting a phylogeny generated with molecular data are difficult to explain. However, high fidelity genome editing technologies like CRISPR/Cas9 are leading to new avenues of study in the evolution of morphological characters. CRISPR/Cas9 has successfully been used to remove the Drosophila Fallen wing vein L2 by deleting a $2 \mathrm{~kb}$ section of the gene regulatory network that controls wing development (Xu et al. 2017), and has been used preliminarily to control expression of $4^{\text {th }}$ sternite appendages in sepsid flies (Su et al. 2017). If the exact genetic mechanism controlling the expression of different genitalic phenotypes could be 
better understood, than some of the puzzling character state reversals implied by a molecular phylogeny may be explained.

\section{References}

Amorim, D. D. S., V. C. Silva and B. V. Brown (2018). "Puyehuemyia chandleri, gen. nov., sp. nov. (Diptera: Opetiidae): Remnant of a Cretaceous Biota in Chile." American Museum Novitates(3892): 1-27.

Andrews, S. (last updated 10 Jan, 2018). "FastQC: a quality control tool for high throughput sequence data. Version 0.11.7." Retrieved 5 March 2018, from https://www.bioinformatics.babraham.ac.uk/projects/fastqc/.

Bank, S., M. Sann, C. Mayer, K. Meusemann, A. Donath, L. Podsiadlowski, A. Kozlov, M. Petersen, L. Krogmann and R. Meier (2017). "Transcriptome and target DNA enrichment sequence data provide new insights into the phylogeny of vespid wasps (Hymenoptera: Aculeata: Vespidae)." Molecular Phylogenetics and Evolution 116: 213-226.

Bolger, A. M., M. Lohse and B. Usadel (2014). "Trimmomatic: a flexible trimmer for Illumina sequence data." Bioinformatics 30(15): 2114-2120.

Chvála, M. (1981). "Classification and phylogeny of Empididae with a presumed origin of Dolichopodidae (Diptera)." Entomologica scandinavica. Supplement 15: $225-236$. 
Collins, K. P. and B. M. Wiegmann (2002). "Phylogenetic relationships of the lower Cyclorrhapha (Diptera: Brachycera) based on 28S rDNA sequences." Insect Systematics and Evolution 33(4): 445-456.

Crampton, G. (1936). "Suggestions for a new interpretation of the postabdomen in male Cyclorrhaphous Diptera." Bulletin of the Brooklyn Entomological Society 31: 141-148.

Cumming, J. M., B. J. Sinclair and D. M. Wood (1995). "Homology and phylogenetic implications of male genitalia in Diptera - Eremoneura." Entomologica Scandinavica 26: 121-151.

Disney, R. (2001). "Sciadoceridae (Diptera) reconsidered." Fragmenta faunistica 44(2): 309-317.

Disney, R. H. L. (1988). "Unusual costal chaetotaxy in the phylogenetically interesting Ironomyiidae and Sciadoceridae (Diptera)." Annales Entomologici Fennici 54: 19-20.

Disney, R. H. L. (1994). "Continuing the debate relating to the phylogenetic reconstruction of the Phoridae (Diptera)." Giornale Italiano di Entomologia 7: 103-117.

Grabherr, M. G., B. J. Haas, M. Yassour, J. Z. Levin, D. A. Thompson, I. Amit, X. Adiconis, L. Fan, R. Raychowdhury and Q. Zeng (2011). "Trinity: reconstructing a fulllength transcriptome without a genome from RNA-Seq data." Nature biotechnology 29(7): 644 . 
Griffiths, G. C. D. (1972). The phylogenetic classification of Diptera Cyclorrhapha with special reference to the structure of the male postabdomen. The Hague, Dr. W. Junk N. V.

Hennig, W. (1973). "31. Diptera (two-winged flies)." Handbuch der Zoologie. Eine Naturgeschichte der Stämme des Tierreiches 4: 1-337.

Hennig, W. (1976). "Das hypopygium von Lonchoptera lutea Panzer und die phylogenetischen verwandtschaftsbeziehungen der Cyclorrhapha (Diptera). [The hypopygium of Lonchoptera lutea Panzer and the phylogenetic affinities of the Cyclorrhapha (Diptera)]." Stuttgarter Beiträge zur Naturkunde aus dem Staatlichen Museum fuer Naturkunde in Stuttgart 283: 1-63.

Kalyaanamoorthy, S., B. Q. Minh, T. K. Wong, A. von Haeseler and L. S. Jermiin (2017). "ModelFinder: fast model selection for accurate phylogenetic estimates." Nature Methods 14: 587-589.

Katoh, K. and D. M. Standley (2013). "MAFFT multiple sequence alignment software version 7: improvements in performance and usability." Molecular biology and evolution 30(4): 772-780.

Kessel, E. L. and E. A. Maggioncalda (1968). "A revision of the genera of Platypezidae, with descriptions of five new genera, and considerations of phylogeny, circumversion, and hypopygia. (Diptera)." The Wasmann journal of biology 26(1): 33-106.

Kluge, Arnold G. (1997). "Sophisticated falsification and research cycles: consequences for differential character weighting in phylogenetic systematics." Zoologica scripta. 26(4): 349-360. 
Kriventseva, E. V., F. Tegenfeldt, T. J. Petty, R. M. Waterhouse, F. A. Simão, I. A. Pozdnyakov, P. Ioannidis and E. M. Zdobnov (2014). "OrthoDB v8: update of the hierarchical catalog of orthologs and the underlying free software." Nucleic acids research 43(D1): D250-D256.

Kück, P., K. Meusemann, J. Dambach, B. Thormann, B. M. von Reumont, J. W. Wägele and B. Misof (2010). "Parametric and non-parametric masking of randomness in sequence alignments can be improved and leads to better resolved trees." Frontiers in zoology 7(1): 10.

Lanfear, R., P. B. Frandsen, A. M. Wright, T. Senfeld and B. Calcott (2016).

"PartitionFinder 2: new methods for selecting partitioned models of evolution for molecular and morphological phylogenetic analyses." Molecular biology and evolution 34(3): 772-773.

McAlpine, J. F. (1989). Phylogeny and classification of the Muscomorpha. Manual of Nearctic Diptera. J. F. McAlpine. Ottawa, Agriculture Canada Monograph No. 32, Research Branch, Agriculture Canada. 3: 1397-1505.

Meyer, B., Meusemann, K., \& Misof, B. (2011). MARE v.0.1.2-rc. Bonn, Germany, Zoological Research Museum Alexander Koenig.

Minh, B. Q., M. A. T. Nguyen and A. von Haeseler (2013). "Ultrafast approximation for phylogenetic bootstrap." Molecular biology and evolution 30(5): 1188-1195.

Mirarab, S. and T. Warnow (2015). "ASTRAL-II: coalescent-based species tree estimation with many hundreds of taxa and thousands of genes." Bioinformatics 31(12): i44-i52. 
Misof, B., S. Liu, K. Meusemann, R. S. Peters, A. Donath, C. Mayer, P. B. Frandsen, J. Ware, T. Flouri and R. G. Beutel (2014). "Phylogenomics resolves the timing and pattern of insect evolution." Science 346(6210): 763-767.

Moulton, J. K. and B. M. Wiegmann (2003). "Evolution and phylogenetic utility of CAD (rudimentary) among Mesozoic-aged Eremoneuran Diptera (Insecta)." Molecular Phylogenetics and Evolution 31: 363-378.

Moulton, J. K. and B. M. Wiegmann (2007). "The phylogenetic relationships of flies in the superfamily Empidoidea (Insecta: Diptera)." Molecular Phylogenetics and Evolution 43: 701-713.

Near, Thomas J. (2009). "Conflict and resolution between phylogenies inferred from molecular and phenotypic data sets for hagfish, lampreys, and gnathostomes." Journal of Experimental Zoology Part B: Molecular and Developmental Evolution. 312(7): 749-761.

Nguyen, L.-T., H. A. Schmidt, A. von Haeseler and B. Q. Minh (2014). "IQ-TREE: a fast and effective stochastic algorithm for estimating maximum-likelihood phylogenies." Molecular biology and evolution 32(1): 268-274.

Pape, T. and F. C. Thompson. (last updated 13 June 2013). "Systema Dipterorum. The Biosystematic Database of World Diptera. Version 1.5." 1.5. Retrieved 5 March 2018, from http://www.diptera.org/.

Pauli, T., T. O. Burt, K. Meusemann, K. Bayless, A. Donath, L. Podsiadlowski, C. Mayer, A. Kozlow, A. Vasilikopoulos, S. Liu, X. Zhou, D. Yeates, D. Misof, R. S. Peters, and X. Mengual. (2018). "New data, same story: phylogenomics does not 
support Syrphoidea (Diptera: Syrphidae, Pipunculidae). Systematic Entomology 12283: 1-13.

Petersen, M., K. Meusemann, A. Donath, D. Dowling, S. Liu, R. S. Peters, L. Podsiadlowski, A. Vasilikopoulos, X. Zhou and B. Misof (2017). "Orthograph: a versatile tool for mapping coding nucleotide sequences to clusters of orthologous genes." BMC bioinformatics 18(1): 111.

Ronquist, F., M. Teslenko, P. Van Der Mark, D. L. Ayres, A. Darling, S. Höhna, B. Larget, L. Liu, M. A. Suchard and J. P. Huelsenbeck (2012). "MrBayes 3.2: efficient Bayesian phylogenetic inference and model choice across a large model space." Systematic biology 61(3): 539-542.

Simon, S., Petersen, M., Bartel, D. and K. Meusemann. (in prep). "1KITE Analyses pipeline - Manual and hands-on. Version 18, 2016-02-12.".

Sinclair, B. J., J. M. Cumming and D. M. Wood (1993). "Homology and phylogenetic implications of male genitalia in Diptera - Lower Brachycera." Entomologica Scandinavica 24(4): 407-432.

Sinclair, B. J. and J. M. Cumming (2006). "The morphology, higher-level phylogeny and classification of the Empidoidea (Diptera)." Zootaxa 1180: 1-172.

Sinclair, B. J. C., J.M.; Brooks, S.E. (2013). "Male terminalia of Diptera (Insecta): a review of evolutionary trends, homology and phylogenetic implications." Insect Systematics and Evolution 44: 373-415.

Stamatakis, A. (2014). "RAxML version 8: a tool for phylogenetic analysis and postanalysis of large phylogenies." Bioinformatics 30(9): 1312-1313. 
Su, K., G. Rajaratnam and R. Meier (2017). "Sex ticklers and dirty flies: The development and evolution of a novel abdominal appendage in male sepsid flies." Mechanisms of Development (145): S21.

Suyama, M., D. Torrents and P. Bork (2006). "PAL2NAL: robust conversion of protein sequence alignments into the corresponding codon alignments." Nucleic acids research 34(suppl 2): W609-W612.

Tachi, T. (2014). "Homology of the metapleuron of Cyclorrhapha, with discussion of the paraphyly of Syrphoidea (Diptera: Aschiza)." Insect Syst Evol: 1-20.

Tkoč, M., A. Tóthová, G. Ståhls, P. J. Chandler and J. Vaňhara (2017). "Molecular phylogeny of flat-footed flies (Diptera: Platypezidae): main clades supported by new morphological evidence." Zoologica Scripta 46(4): 429-444.

Wiegmann, B. M., M. D. Trautwein, I. S. Winkler, N. B. Barr, J.-W. Kim, C. Lambkin, M. A. Bertone, B. K. Cassel, K. M. Bayless, A. M. Heimberg, B. M. Wheeler, K. J. Peterson, T. Pape, B. J. Sinclair, J. H. Skevington, V. Blagoderov, J. Caravask, S. N. Kutty, U. Schmidt-Ott, G. E. Kampmeier, F. C. Thompson, D. A. Grimaldi, A. T. Beckenbach, G. W. Courtney, M. Friedrich, R. Meier and D. K. Yeates (2011). "Episodic radiations in the fly tree of life." Proc Natl Acad Sci U S A 108(14): $5690-5695$.

Winterton, S. L., A. R. Lemmon, J. P. Gillung, I. J. Garzon, D. Badano, D. K. Bakkes, L. C. Breitkreuz, M. S. Engel, E. M. Lemmon and X. Liu. (2017). "Evolution of lacewings and allied orders using anchored phylogenomics (Neuroptera, Megaloptera, Raphidioptera)." Systematic Entomology 43(2): 330-354. 
Woodley, N. E. (1989). Phylogeny and classification of the 'Orthorrhaphous' Brachycera. [Chapter] 115. Manual of Nearctic Diptera. J. F. McAlpine and D. M. Wood, Agriculture Canada Monograph 32. 3: 1371-1395.

Xie, Y., G. Wu, J. Tang, R. Luo, J. Patterson, S. Liu, W. Huang, G. He, S. Gu and S. Li (2014). "SOAPdenovo-Trans: de novo transcriptome assembly with short RNA-Seq reads." Bioinformatics 30(12): 1660-1666.

Xu, X.-R. S., V. M. Gantz, N. Siomava and E. Bier (2017). "CRISPR/Cas9 and Active Genetics-based trans-species replacement of the endogenous Drosophila kniL2 CRM reveals unexpected complexity." eLife 6: e30281.

Young, A. D., A. R. Lemmon, J. H. Skevington, X. Mengual, G. Ståhls, M. Reemer, K. Jordaens, S. Kelso, E. M. Lemmon, M. Hauser, M. De Meyer, B. Misof and B. Wiegmann (2016). "Anchored enrichment dataset for true flies (order Diptera) reveals insights into the phylogeny of flower flies (family Syrphidae)." BMC Evolutionary Biology 16(1): 143.

Zatwarnicki, T. (1996). "A new reconstruction of the origin of eremoneuran hypopygium and its implications for classification (Insecta: Diptera)." Genus 7(1): 103-175. 
Chapter 1, Table 1: Taxa used for analyses, original sequencing locations (source), NCBI (National Center for Biotechnology Information) IDs, if published, and collection locality when known. FSU = Florida State University, NCSU = North Carolina State University.

\begin{tabular}{|c|c|c|c|c|c|c|}
\hline Family & Genus & Species & Source & ID (NCBI) & Locality & Accession \\
\hline Asilidae & Zosteria & rosevillensis & 1KITE & INSerlTAYRAAPEI-22 & Australia & \\
\hline Bombyliidae & Bombylius & major & 1KITE & INSbusTBKRAAPEI-76 & USA & \\
\hline Therevidae & Neodialineura & nitens & $1 \mathrm{KITE}$ & INSerlTBNRAAPEI-61 & Australia & \\
\hline Atelestidae & Meghyperus & sp. & NCSU & & USA & \\
\hline Hybotidae & Leptopeza & sp. & $1 \mathrm{KITE}$ & WHANIsrmTMALRAAPEI-22 & USA & \\
\hline Dolichopodidae & Argyra & sp. & FSU & & Tennessee, USA & JSS 22748 \\
\hline Dolichopodidae & Condostylus & patibulatus & NCBI & SRR5559330 SRR5559329 & unknown & \\
\hline Empididae & Empis & snoddyi & NCSU & & USA & \\
\hline Empididae & Iteaphila & sp. & FSU & & Ontario, Canada & JSS 23238 \\
\hline Brachystomatidae & Brachystoma & occidentalis & NCSU & & USA & \\
\hline Oreogetonidae & Oreogeton & $\mathrm{sp}$. & NCSU & & USA & \\
\hline Apystomyiidae & Apystomyia & elinguis & NCSU & & California, USA & JSS 17011 \\
\hline Lonchopteridae & Lonchoptera & bifurcata & NCSU & & Oregon, USA & CNCD 1443 \\
\hline Opetiidae & Opetia & nigra & NCSU & & $\begin{array}{l}\text { Weigmann et al. } \\
2011\end{array}$ & \\
\hline Platypezidae & Microsania & sp. & NCSU & & Brazil & \\
\hline Platypezidae & Agathomyia & viduella & $\mathrm{NCSU}$ & & Germany & \\
\hline Platypezidae & Platypeza & anthrax & 1KITE & RINSinlTBORAAPEI-22 & USA & \\
\hline Platypezidae & Platypeza & sp. & FSU & SRX1131540 & Ontario, Canada & JSS 24755 \\
\hline Platypezidae & Calotarsa & sp. & NCSU & calosaRG & South Africa & \\
\hline Platypezidae & Lindneromyia & sp. & FSU & & Oregon, USA & CNCD 1442 \\
\hline Ironomyiidae & Ironomyia & francisi & FSU & & Queensland, AU & JSS 22757 \\
\hline Ironomyiidae & Ironomyia & nigromaculata & FSU & & Queensland, AU & JSS 22756 \\
\hline Phoridae & Sciadocera & sp. & $\mathrm{NCSU}$ & & Australia & \\
\hline Phoridae & Anevrina & sp. & FSU & & Quebec, Canada & JSS 23237 \\
\hline Phoridae & Megaselia & abdita & $1 \mathrm{KITE}$ & RINSinlTBGRAAPEI-126 & $\begin{array}{l}\text { German lab } \\
\text { culture }\end{array}$ & \\
\hline
\end{tabular}


Chapter 1, Table 1 (conc'l): Taxa used for analyses, original sequencing locations, NCBI (National Center for Biotechnology Information) IDs if published, and collection locality when known. FSU = Florida State University, NCSU = North Carolina State University.

\begin{tabular}{|c|c|c|c|c|c|c|}
\hline Family & Genus & Species & Source & ID (NCBI) & Locality & Accession \\
\hline Pipunculidae & Nephrocerus & atrapilus & $1 \mathrm{KITE}$ & INSobdTEHRAAPEI-44 & USA & \\
\hline Pipunculidae & Chalarus & spurius & Young et al 2016 & SRX1131538 & Granada, Spain & JSS 22746 \\
\hline Pipunculidae & Pipunculus & sp. 0N12 & Young et al 2016 & SRX1131539 & Ontario, Canada & JSS 24663 \\
\hline Conopidae & Sicus & ferrugineus & $1 \mathrm{KITE}$ & INShkeTARRAAPEI-46 & Germany & \\
\hline Lauxaniidae & Sapromyza & sciomyzina & $1 \mathrm{KITE}$ & INSerlTATRABPEI-16 & Australia & \\
\hline Sciomyzidae & Limnia & unguicornis & $1 \mathrm{KITE}$ & INSlupTAURAAPEI-94 & Germany & \\
\hline Tephritidae & Ceratitis & capitata & NCBI & PRJNA208956 & unknown & \\
\hline Drosophilidae & Drosophila & melanogaster & NCBI & OrthoDB & unknown & \\
\hline Tachinidae & Epalpus & signifer & Young et al 2016 & SRC1131537 & Quebec, Canada & JSS 23233 \\
\hline Syrphidae & Archimicrodon & brachycerus & 1KITE & INShkeTBBRAAPEI-57 & Australia & \\
\hline Syrphidae & Eumerus & sp. & Young et al 2016 & SRX1131518 & Extremadura, Spain & JSS 22745 \\
\hline Syrphidae & Helophilus & fasciatus & Young et al 2016 & SRX1131515 & Ontario, Canada & JSS 23235 \\
\hline Syrphidae & Ferdinandea & buccata & Young et al 2016 & SRX1131534 & Tennessee, USA & JSS 26304 \\
\hline Syrphidae & Heringia & sp. & Young et al 2016 & SRX1131508 & Quebec, Canada & JSS 22754 \\
\hline Syrphidae & Pipiza & nigripilosa & Young et al 2016 & SRX1131513 & North Carolina, USA & JSS 22762 \\
\hline Syrphidae & Melanostoma & mellinum & Young et al 2016 & SRX1131529 & Ontario, Canada & JSS 24699 \\
\hline Syrphidae & Allograpta & obliqua & Young et al 2016 & SRX1131530 & Mississippi, USA & JSS 26309 \\
\hline Syrphidae & Leucozona & americanum & Young et al 2016 & SRX1131507 & Quebec, Canada & JSS 23231 \\
\hline
\end{tabular}


Chapter 1, Table 2: fasta2hypo group designations

\begin{tabular}{|c|c|c|c|}
\hline Taxon & Group & Taxon & Group \\
\hline Zosteria rosevillensis & Group 1 & Sciadocera sp. & Group 4 \\
\hline Bombylius major & Group 1 & Anevrina sp. & Group 4 \\
\hline Neodialineura nitens & Group 1 & Megaselia abdita & Group 4 \\
\hline Meghyperus sp. & Group 2 & Nephrocerus atrapilus & Group 5 \\
\hline Leptopeza sp. & Group 2 & Chalarus spurius & Group 5 \\
\hline Argyra sp. & Group 2 & Pipunculus sp. ON12 & Group 5 \\
\hline Condostylus patibulatus & Group 2 & Sicus ferrugineus & Group 6 \\
\hline Empis snoddyi & Group 2 & Sapromyza sciomyzina & Group 6 \\
\hline Iteaphila sp. & Group 2 & Limnia unguicornis & Group 6 \\
\hline Brachystoma occidentalis & Group 2 & Ceratitis capitata & Group 7 \\
\hline Oreogeton sp. & Group 2 & Drosophila melanogaster & Group 7 \\
\hline Apystomyia elinguis & Group 2 & Epalpus signifer & Group 7 \\
\hline Lonchoptera bifurcata & Group 3 & Archimicrodon brachycerus & Group 8 \\
\hline Opetia nigra & Group 3 & Eumerus sp. & Group 8 \\
\hline Microsania sp. & Group 3 & Helophilus fasciatus & Group 8 \\
\hline Agathomyia viduella & Group 3 & Ferdinandea buccata & Group 8 \\
\hline Platypeza anthrax & Group 3 & Heringia sp. & Group 8 \\
\hline Platypeza sp. & Group 3 & Pipiza nigripilosa & Group 8 \\
\hline Calotarsa sp. & Group 3 & Melanostoma mellinum & Group 8 \\
\hline Lindneromyia sp. & Group 3 & Allograpta obliqua & Group 8 \\
\hline Ironomyia francisi & Group 4 & Leucozona americanum & Group 8 \\
\hline Ironomyia nigromaculata & Group 4 & & \\
\hline
\end{tabular}


Chapter 1, Table 3: Analyses performed and summary statistics

\begin{tabular}{|c|c|c|c|c|c|c|c|}
\hline Dataset & Figure number & Partitioning & Analysis tool & $\%$ missing data & $\begin{array}{l}\text { \# characters } \\
\text { (aa/nts) }\end{array}$ & $\begin{array}{l}\text { Final ML } \\
\text { optimization }\end{array}$ & $\begin{array}{l}\text { Avg deviation } \\
\text { of split freqs } \\
\text { (MrBayes) }\end{array}$ \\
\hline $\begin{array}{l}2446 \text { locus } \\
\text { dataset (nt } \\
\text { alignment) }\end{array}$ & 3 & $\begin{array}{l}\text { per-gene } \\
\text { partitions (per- } \\
\text { gene model of } \\
\text { evolution in } \\
\text { ModelFinder) }\end{array}$ & IQ-TREE & $63.32 \%$ & 3412089 & -43541838.45 & - \\
\hline $\begin{array}{l}\text { AHE dataset (nt } \\
\text { alignment) }\end{array}$ & 4 & $\begin{array}{l}\text { IQ-TREE } \\
\text { Modeltest } \\
\text { metapartitions }\end{array}$ & IQ-TREE & $38.88 \%$ & 418731 & -6266430.352 & - \\
\hline $\begin{array}{l}2446 \text { locus } \\
\text { dataset (aa } \\
\text { alignment) }\end{array}$ & 5 & $\begin{array}{l}\text { per-gene } \\
\text { partitions (per- } \\
\text { gene model of } \\
\text { evolution in } \\
\text { ModelFinder) }\end{array}$ & IQ-TREE & $63.32 \%$ & 1111144 & -14551485.05 & - \\
\hline $\begin{array}{l}\text { AHE dataset (aa } \\
\text { alignment) }\end{array}$ & 6 & $\begin{array}{l}\text { IQ-TREE } \\
\text { Modeltest } \\
\text { metapartitions }\end{array}$ & IQ-TREE & $38.88 \%$ & 139577 & -1846768.388 & - \\
\hline $\begin{array}{l}2446 \text { locus } \\
\text { dataset (nt } \\
\text { alignment) }\end{array}$ & 7 & $\begin{array}{l}\text { per-gene } \\
\text { partitions }\end{array}$ & RAxML & $63.32 \%$ & 3412089 & -39249122.28 & - \\
\hline $\begin{array}{l}\text { AHE dataset (nt } \\
\text { alignment) }\end{array}$ & 8 & $\begin{array}{l}\text { PartitionFinder2 } \\
\text { metapartitions }\end{array}$ & RAxML & $38.88 \%$ & 418731 & -6287594.249 & - \\
\hline $\begin{array}{l}\text { AHE dataset (nt } \\
\text { alignment) }\end{array}$ & 9 & - & ASTRAL-II & $38.88 \%$ & 418731 & - & - \\
\hline $\begin{array}{l}\text { AHE dataset (nt } \\
\text { alignment) }\end{array}$ & 10 & $\begin{array}{l}\text { PartitionFinder2 } \\
\text { metapartitions }\end{array}$ & MrBayes & $38.88 \%$ & 418731 & - & 0.034493 \\
\hline $\begin{array}{l}\text { AHE dataset (aa } \\
\text { alignment) }\end{array}$ & 11 & $\begin{array}{l}\text { IQ-TREE } \\
\text { Modeltest } \\
\text { metapartitions }\end{array}$ & MrBayes & $38.88 \%$ & 139577 & - & 0.0 \\
\hline
\end{tabular}


Chapter 1, Table 4: Summary of relationships recovered from each analysis

\begin{tabular}{|c|c|c|c|c|c|c|c|c|c|}
\hline Analysis & Figure & Empidoidea & $\begin{array}{l}\text { Apystomyiidae } \\
+ \\
\text { Cyclorrhapha }\end{array}$ & Lonchopteridae & $\begin{array}{l}\text { Ironomyiidae } \\
\text { + Phoridae }\end{array}$ & $\begin{array}{l}\text { Opetiidae + } \\
\text { Microsania }\end{array}$ & Platypezidae & $\begin{array}{l}\text { (Platypezidae + } \\
\text { Opetiidae) + } \\
\text { (Ironomyiidae + } \\
\text { Phoridae) } \\
\end{array}$ & $\begin{array}{l}\text { Pipunculidae } \\
\text { + Schizophora }\end{array}$ \\
\hline $\begin{array}{l}\text { IQ-TREE } \\
2446 \text { locus } \\
\text { dataset (nts) }\end{array}$ & 3 & monophyletic & monophyletic & $\begin{array}{l}\text { Sister to } \\
\text { Cyclorrhapha }\end{array}$ & monophyletic & $\begin{array}{l}\text { monophyletic, } \\
\text { sister to } \\
\text { Platypezidae }\end{array}$ & nonmonophyletic & monophyletic & monophyletic \\
\hline $\begin{array}{l}\text { IQ-TREE AHE } \\
\text { dataset (nts) }\end{array}$ & 4 & monophyletic & monophyletic & $\begin{array}{l}\text { Sister to } \\
\text { Cyclorrhapha }\end{array}$ & monophyletic & $\begin{array}{l}\text { monophyletic, } \\
\text { sister to } \\
\text { Platypezidae }\end{array}$ & nonmonophyletic & nonmonophyletic & monophyletic \\
\hline $\begin{array}{l}\text { IQ-TREE } \\
2446 \text { locus } \\
\text { dataset (aas) }\end{array}$ & 5 & monophyletic & monophyletic & $\begin{array}{l}\text { Sister to } \\
\text { Ironomyiidae + } \\
\text { Phoridae }+ \\
\text { Opetiidae }+ \\
\text { Platypezidae } \\
\end{array}$ & monophyletic & $\begin{array}{l}\text { monophyletic, } \\
\text { sister to } \\
\text { Platypezidae + } \\
\text { Ironomyiidae + } \\
\text { Phoridae } \\
\end{array}$ & nonmonophyletic & monophyletic & monophyletic \\
\hline $\begin{array}{l}\text { IQ-TREE AHE } \\
\text { dataset (aas) }\end{array}$ & 6 & monophyletic & monophyletic & $\begin{array}{l}\text { Sister to } \\
\text { Ironomyiidae + } \\
\text { Phoridae }+ \\
\text { Opetiidae }+ \\
\text { Platypezidae }\end{array}$ & monophyletic & $\begin{array}{l}\text { monophyletic, } \\
\text { sister to } \\
\text { Platypezidae + } \\
\text { Ironomyiidae + } \\
\text { Phoridae }\end{array}$ & nonmonophyletic & monophyletic & monophyletic \\
\hline $\begin{array}{l}\text { RAxML } 2446 \\
\text { locus dataset } \\
\text { (nts) }\end{array}$ & 7 & monophyletic & monophyletic & $\begin{array}{l}\text { Sister to } \\
\text { remainder of } \\
\text { Cyclorrhapha }\end{array}$ & monophyletic & $\begin{array}{l}\text { monophyletic, } \\
\text { sister to } \\
\text { Platypezidae }\end{array}$ & nonmonophyletic & monophyletic & monophyletic \\
\hline $\begin{array}{l}\text { RAxML AHE } \\
\text { dataset (nts) }\end{array}$ & 8 & monophyletic & monophyletic & $\begin{array}{l}\text { Sister to } \\
\text { remainder of } \\
\text { Cyclorrhapha }\end{array}$ & monophyletic & $\begin{array}{l}\text { monophyletic, } \\
\text { sister to } \\
\text { Platypezidae }\end{array}$ & nonmonophyletic & nonmonophyletic & monophyletic \\
\hline $\begin{array}{l}\text { ASTRAL-II } \\
\text { AHE dataset } \\
\text { (nts) }\end{array}$ & 9 & monophyletic & monophyletic & $\begin{array}{l}\text { Sister to } \\
\text { remainder of } \\
\text { Cyclorrhapha }\end{array}$ & monophyletic & $\begin{array}{l}\text { nonmonophyletic, } \\
\text { Opetiidae alone } \\
\text { sister to } \\
\text { Platypezidae }\end{array}$ & monophyletic & nonmonophyletic & monophyletic \\
\hline $\begin{array}{l}\text { MrBayes } \\
\text { AHE dataset } \\
\text { (nts) }\end{array}$ & 10 & monophyletic & monophyletic & $\begin{array}{l}\text { Sister to } \\
\text { remainder of } \\
\text { Cyclorrhapha }\end{array}$ & monophyletic & $\begin{array}{l}\text { monophyletic, } \\
\text { sister to } \\
\text { Platypezidae }\end{array}$ & nonmonophyletic & nonmonophyletic & monophyletic \\
\hline $\begin{array}{l}\text { MrBayes } \\
\text { AHE dataset } \\
\text { (aas) }\end{array}$ & 11 & monophyletic & monophyletic & $\begin{array}{l}\text { Sister to } \\
\text { Ironomyiidae + } \\
\text { Phoridae }+ \\
\text { Opetiidae + } \\
\text { Platypezidae }\end{array}$ & monophyletic & $\begin{array}{l}\text { monophyletic, } \\
\text { sister to } \\
\text { Platypezidae + } \\
\text { Ironomyiidae + } \\
\text { Phoridae } \\
\end{array}$ & nonmonophyletic & monophyletic & monophyletic \\
\hline
\end{tabular}




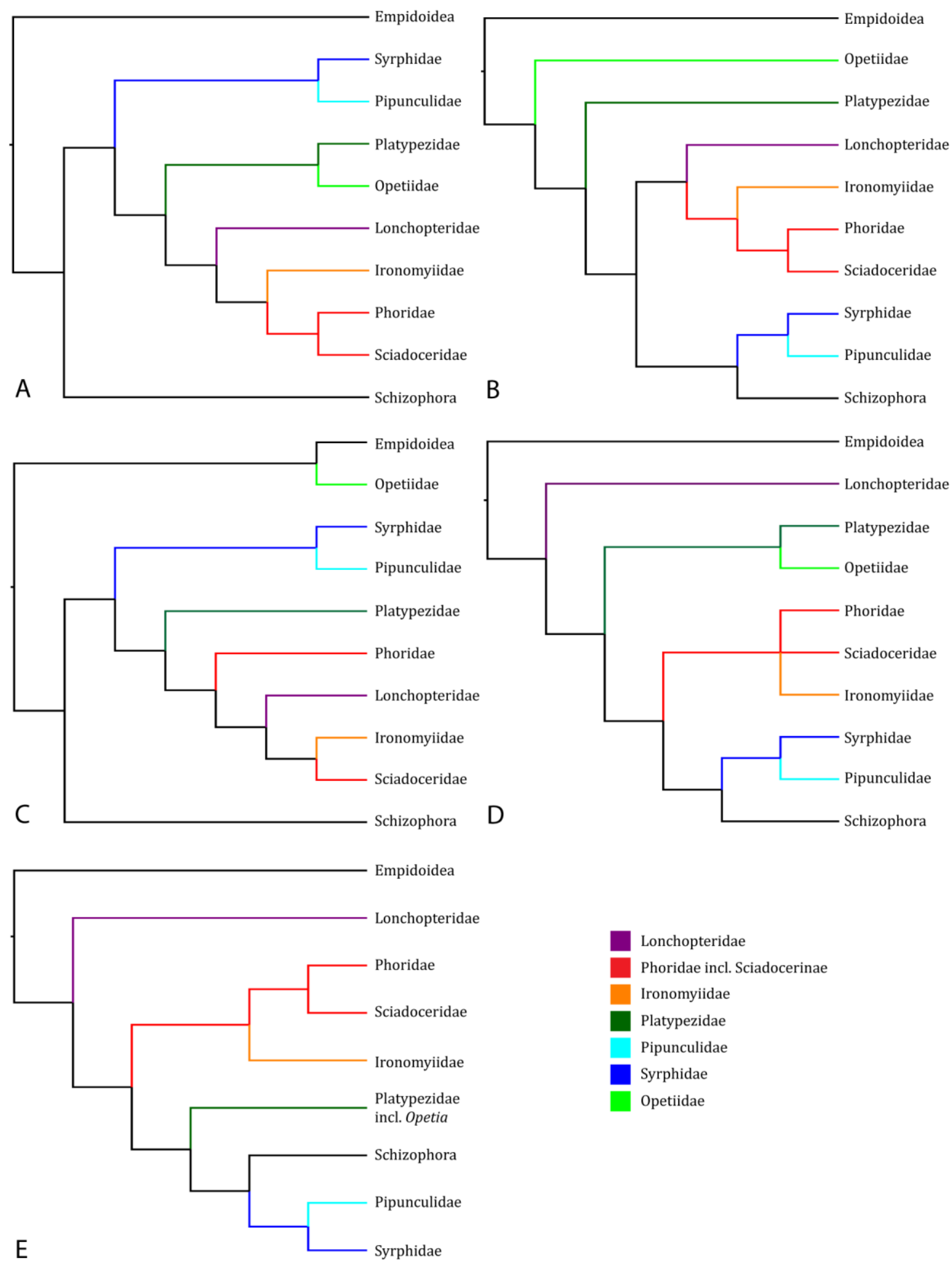

Chapter 1, Figure 1. Alternate phylogenetic hypotheses recovered with morphological character data by different authors. A) McAlpine (1989), supported by Brown $(1992,1995)$. B) Cumming et al. (1995). C) Disney (1994). D) Zatwarnicki (1996). E) Griffiths (1972). 


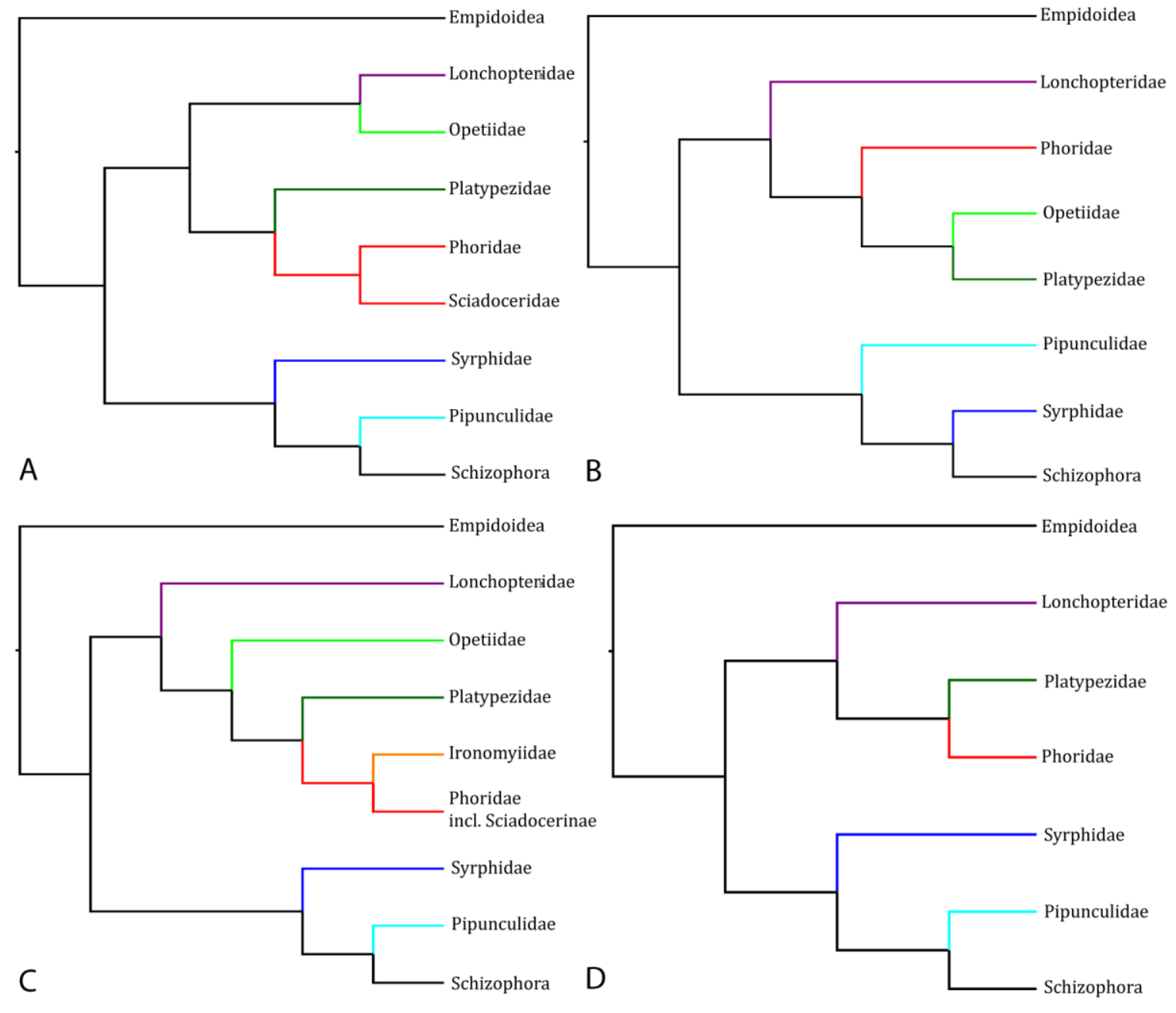

Lonchopteridae

Phoridae incl. Sciadocerinae

Ironomyiidae

Platypezidae

Pipunculidae

Syrphidae

Opetiidae

Chapter 1, Figure 2. Phylogenetic hypotheses recovered with molecular data by different authors. A) Collins and Wiegmann (2002) using 28S rDNA. B) Moulton and Wiegmann (2003) using CAD. C) Wiegmann et al. (2011) using 14 nuclear loci, the mitochondrial genome, and 371 morphological characters. D) Pauli et al. (2018) using transcriptomic data. 


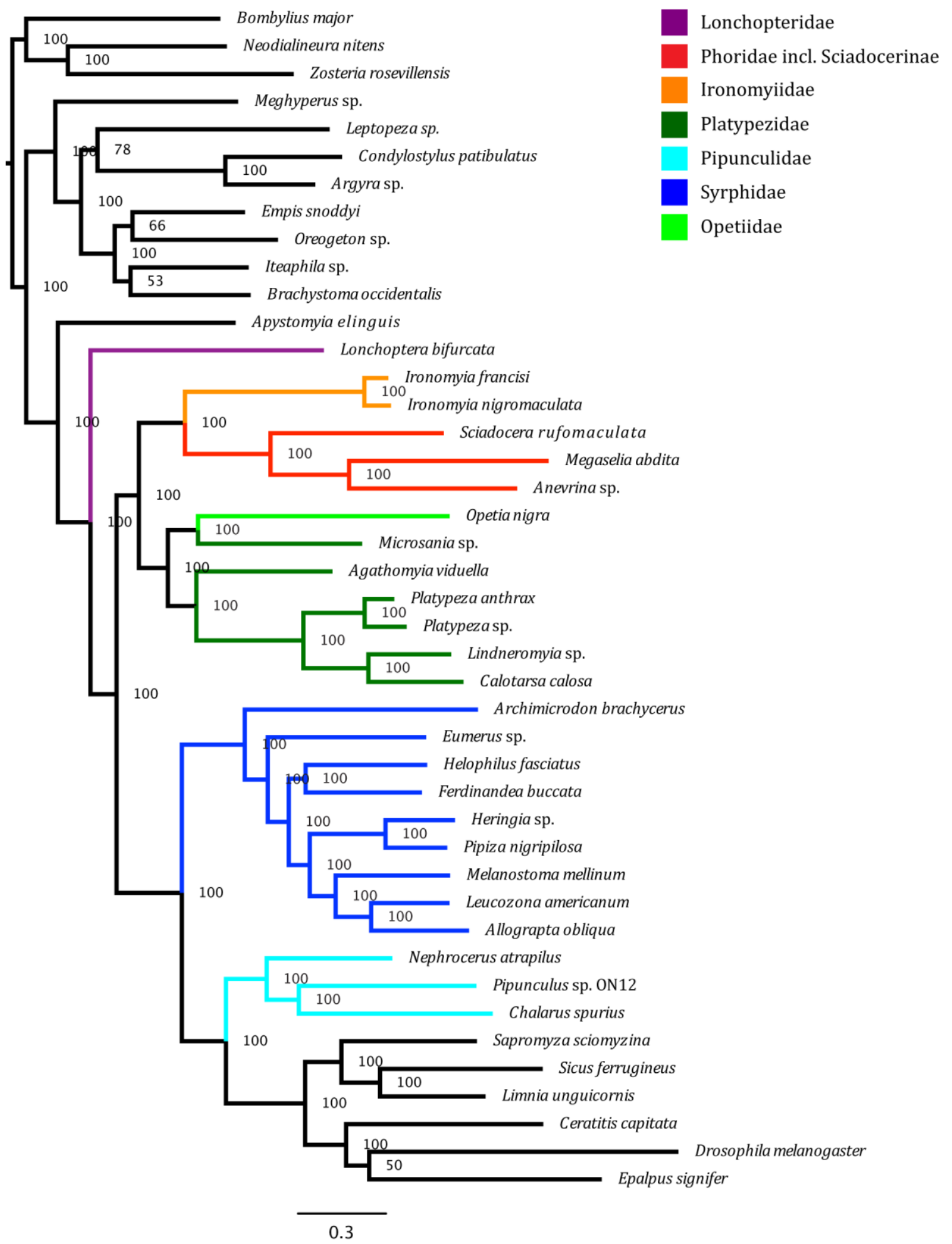

Chapter 1, Figure 3. Maximum likelihood phylogeny recovered through analysis of the full nucleotide dataset, using IQ-TREE. Partitioning was by-gene, with the most appropriate model of evolution for each gene calculated using IQ-TREE's ModelFinder. 1000 Ultrafast Bootstraps were performed, with results printed to the right of each node. 


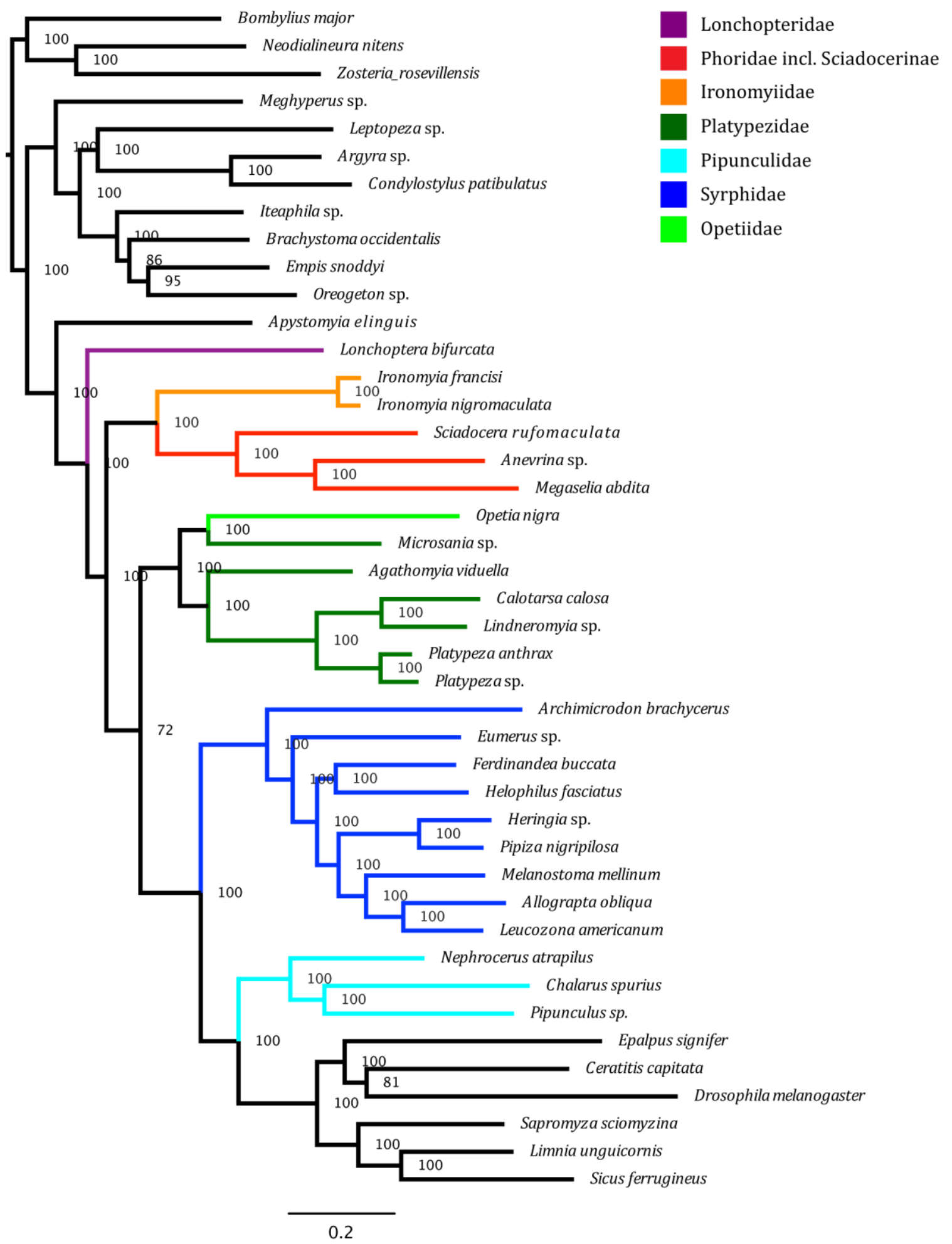

Chapter 1, Figure 4. Maximum likelihood phylogeny recovered through analysis of AHE nucleotide dataset, using IQ-TREE. Metapartitions and model selection were calculated using IQ-TREE's Modelfinder. 1000 Ultrafast Bootstraps were performed, with results printed to the right of each node. 


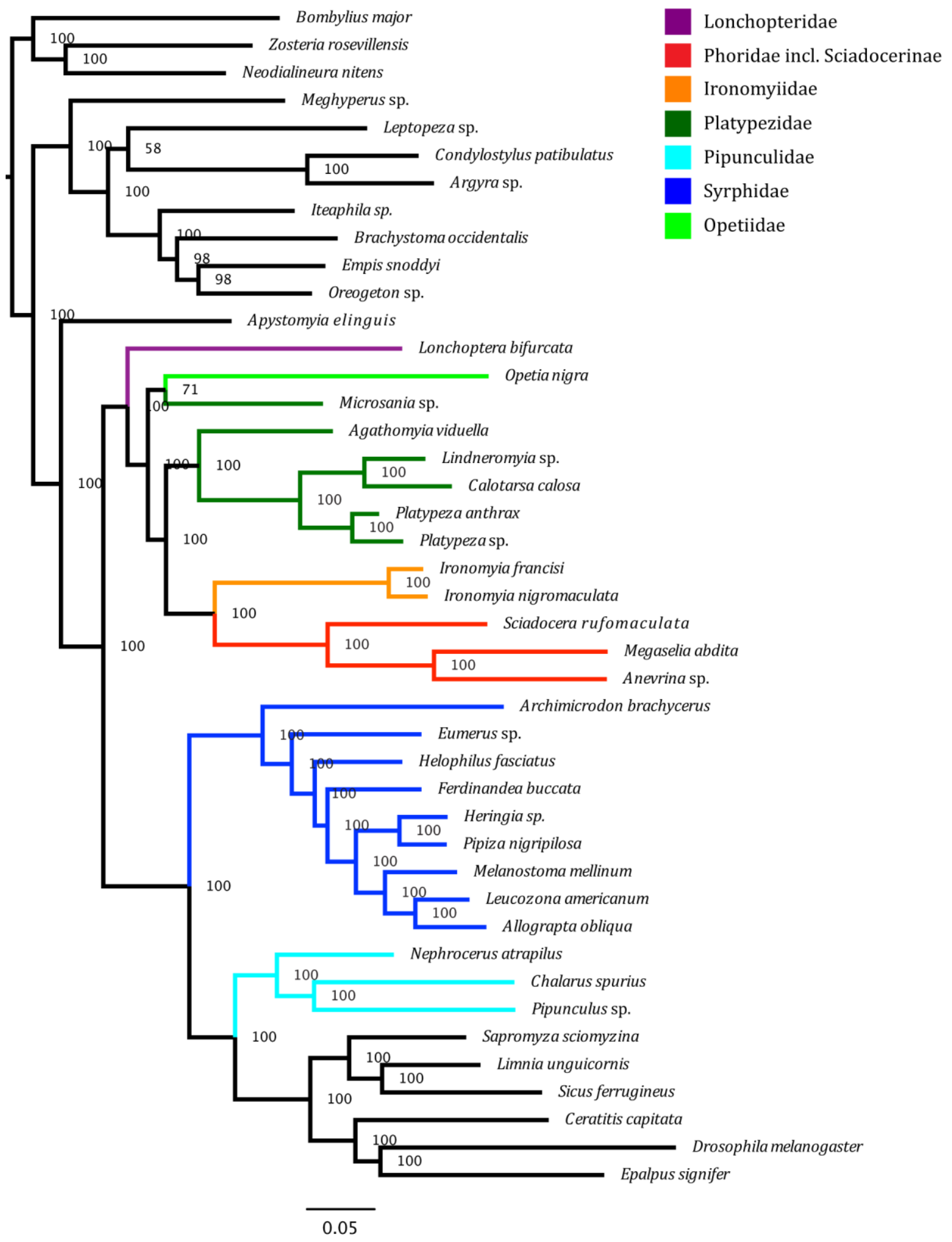

Chapter 1, Figure 5. Maximum likelihood phylogeny recovered through analysis of the full amino acid dataset, using IQ-TREE. Partitioning was by-gene, with the most appropriate model of evolution for each gene calculated using IQ-TREE's ModelFinder. 1000 Ultrafast Bootstraps were performed, with results printed to the right of each node. 


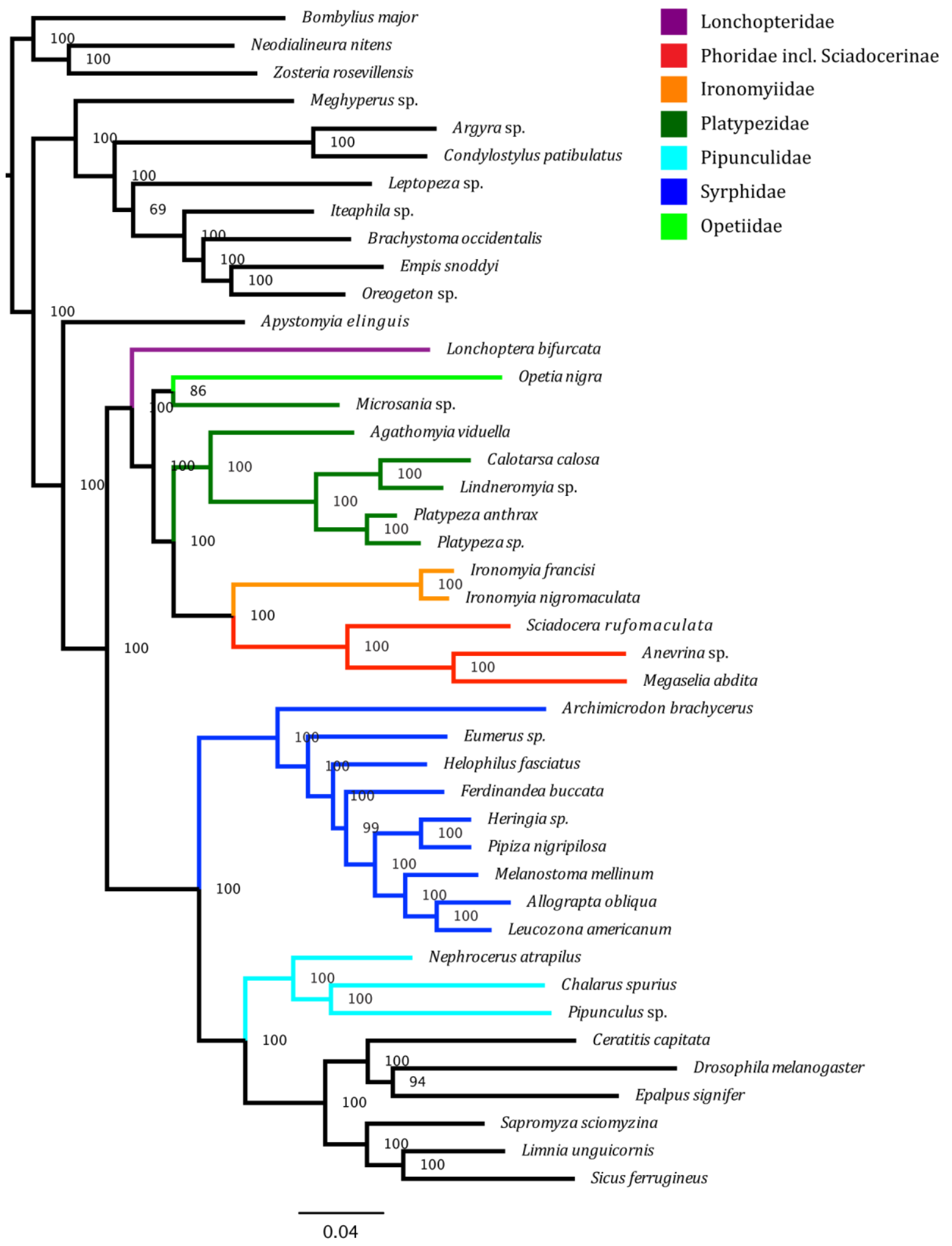

Chapter 1, Figure 6. Maximum likelihood phylogeny recovered through analysis of AHE amino acid dataset, using IQ-TREE. Metapartitions and model selection were calculated using IQ-TREE's Modelfinder. 1000 Ultrafast Bootstraps were performed, with results printed to the right of each node. 


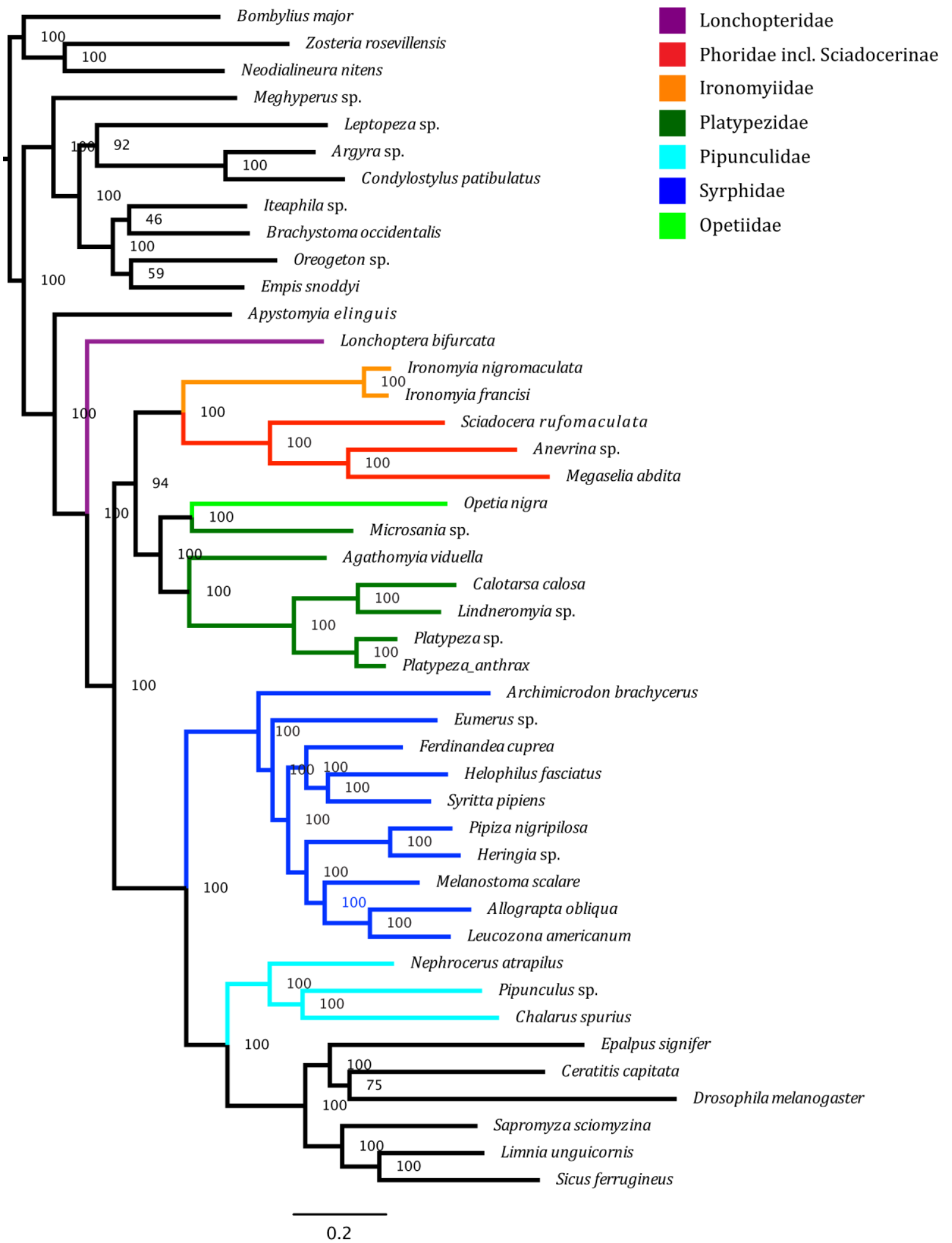

Chapter 1, Figure 7. Maximum likelihood phylogeny recovered through analysis of full nucleotide dataset, using RAxML. Partitioning was by-gene, using the GTR model of evolution. 100 bootstraps were performed, with results printed to the right of each node. 


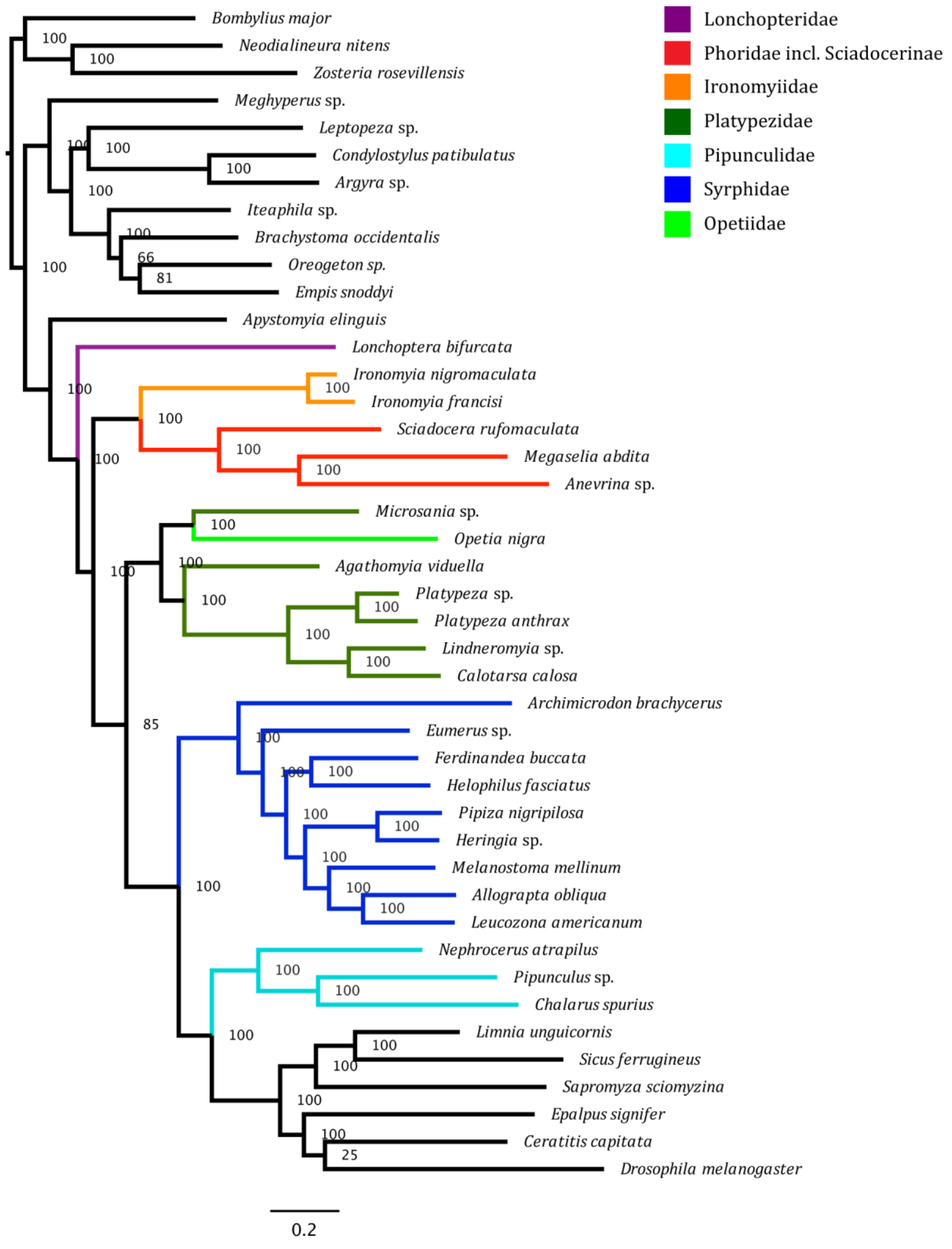

Chapter 1, Figure 8. Maximum likelihood phylogeny recovered through analysis of AHE nucleotide dataset, using RAxML. Metapartitions were calculated using PartitionFinder2, and the GTR model of evolution was used. 100 bootstraps were performed, with results printed to the right of each node. 


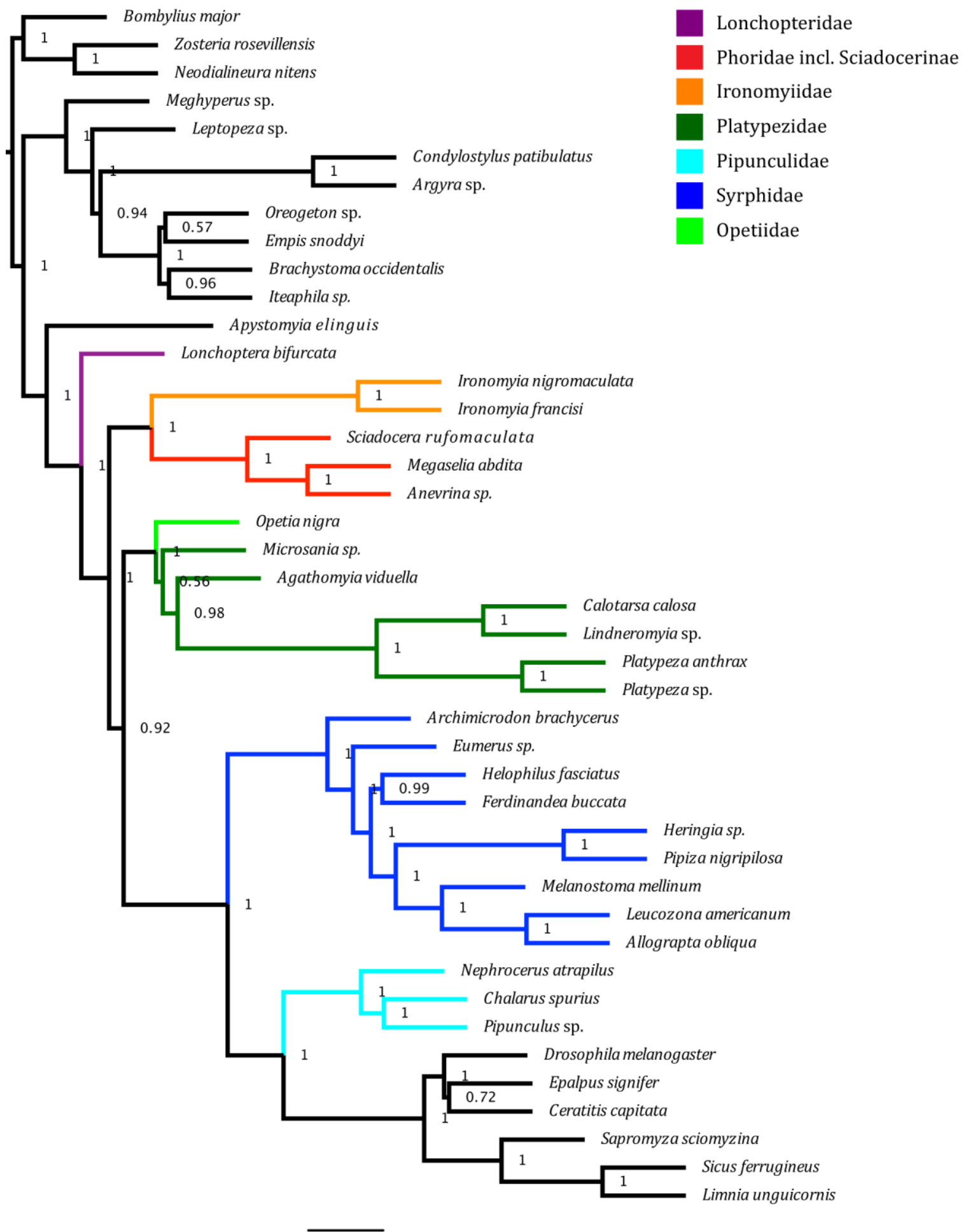

0.9

Chapter 1, Figure 9. Coalescent modeling phylogeny recovered through analysis of AHE nucleotide dataset, using ASTRAL-II. Individual gene trees were calculated in RAxML as input for ASTRAL-II. 


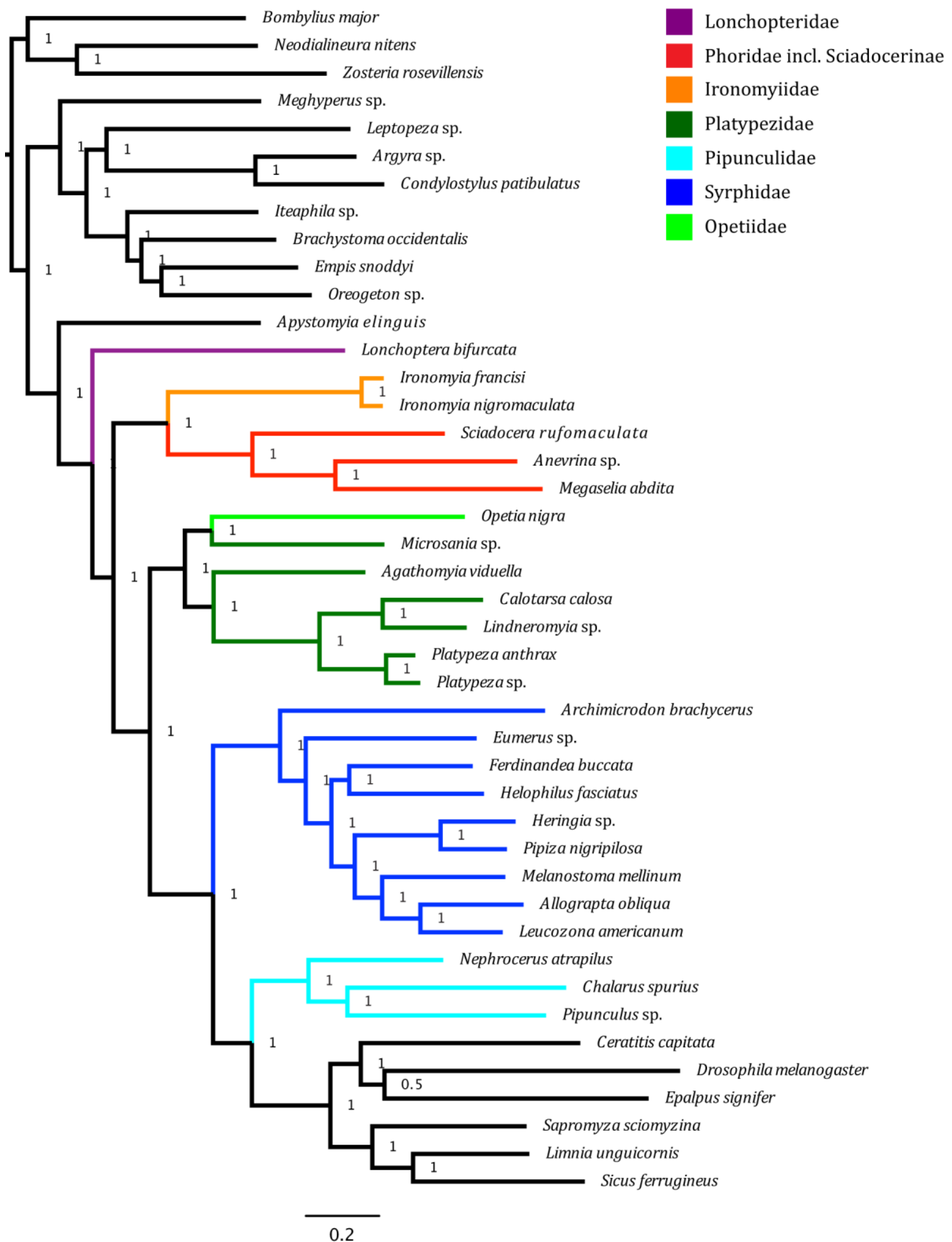

Chapter 1, Figure 10. Bayesian phylogeny recovered through analysis of AHE nucleotide dataset, using MrBayes 3.2.6. The analysis was run for 10 million generations. Posterior probabilities are printed to the right of each node. 


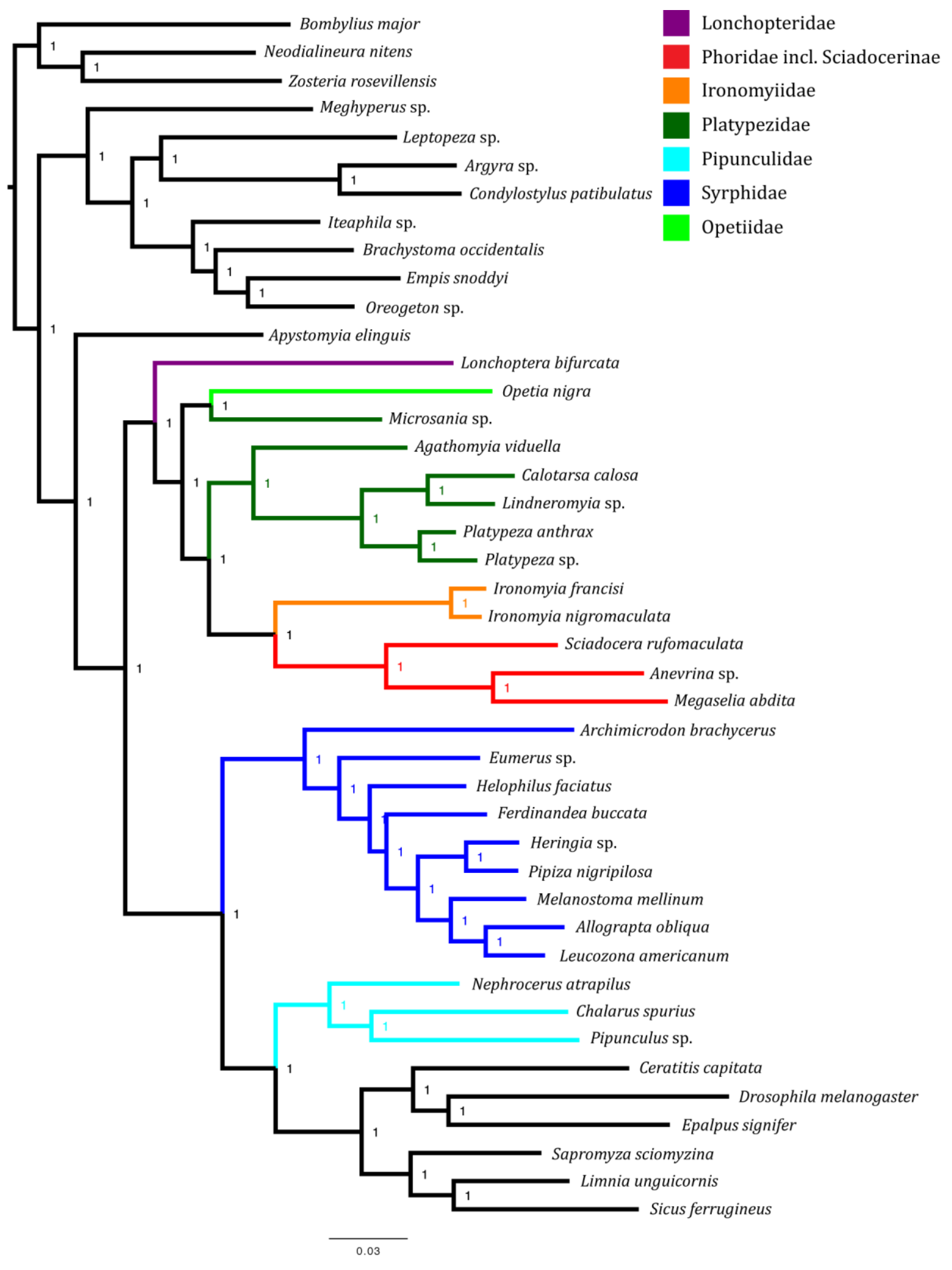

Chapter 1, Figure 11. Bayesian phylogeny recovered through analysis of AHE amino acid dataset, using MrBayes 3.2.6. The analysis was run for 5 million generations. Posterior probabilities are printed to the right of each node. 


\section{Chapter 2: Anchored enrichment dataset for true flies (order Diptera) reveals insights into the phylogeny of flower flies (family Syrphidae)}

Young, Andrew Donovan, Lemmon, Alan R., Skevington, Jeffrey H., Mengual, Ximo, Ståhls, Gunilla, Reemer, Menno, Jordaens, Kurt, Kelso, Scott, Lemmon, Emily Moriarty, Hauser, Martin, De Meyer, Marc, Misof, Bernhard, Wiegmann, Brian M.

Publication note: This chapter is available in peer-reviewed, open access format in BMC Evolutionary Biology at the following URL:

https://bmcevolbiol.biomedcentral.com/articles/10.1186/s12862-016-0714-0 and can be cited as: Young, A. D., A. R. Lemmon, J. H. Skevington, X. Mengual, G. Ståhls, M.

Reemer, K. Jordaens, S. Kelso, E. M. Lemmon, M. Hauser, M. De Meyer, B. Misof and B. Wiegmann (2016). "Anchored enrichment dataset for true flies (order Diptera) reveals insights into the phylogeny of flower flies (family Syrphidae)." BMC Evolutionary Biology 16(1): 143.

\section{Author contributions:}

Young, Andrew D.: manuscript assembly, manuscript revisions, voucher identification, wrote introduction, results, discussion Lemmon, A.R.: probe design, lab work oversight, analysis, manuscript revisions, wrote methods Skevington, Jeffrey H.: project conceptualization, manuscript coordination, voucher 
collection, voucher identification, manuscript revisions, phylogenetic and taxonomic insights

Mengual, X.: project conceptualization, manuscript revisions, voucher collection, phylogenetic and taxonomic insights, Figure 1.

Ståhls, G.: project conceptualization, manuscript revisions, voucher collection, phylogenetic and taxonomic insights

Reemer, M.: project conceptualization, manuscript revisions, voucher collection, taxonomic insights

Jordaens, K.: project conceptualization, manuscript revisions, phylogenetic insights Kelso, S.: manuscript revisions, analysis, molecular lab work \& COI sequencing

Lemmon, E. Moriarty: manuscript revisions, lab work

Hauser, M.: project conceptualization, manuscript revisions, taxonomic insights

De Meyer, M.: project conceptualization, manuscript revisions

Misof, B.: manuscript revisions, probe development

Wiegmann, Brian M.: manuscript revisions, probe coordination and development

\begin{abstract}
Background:

Anchored hybrid enrichment is a form of next-generation sequencing that uses oligonucleotide probes to target conserved regions of the genome flanked by less conserved regions in order to acquire data useful for phylogenetic inference from a broad range of taxa. Once a probe kit is developed, anchored hybrid enrichment is superior to traditional PCR-based Sanger sequencing in terms of both
\end{abstract}


the amount of genomic data that can be recovered and effective cost. Owing to their incredibly diverse nature, importance as pollinators, and historical instability with regards to subfamilial and tribal classification, Syrphidae (flower flies or hoverflies) are an ideal candidate for anchored hybrid enrichment-based phylogenetics, especially since recent molecular phylogenies of the syrphids using only a few markers have resulted in highly unresolved topologies. Over 6,200 syrphid species are currently known and uncovering their phylogeny will help us to understand how these taxa have diversified, providing insight into an array of ecological processes, from the development of adult mimicry, the origin of adult migration, to pollination patterns and the evolution of larval resource utilization.

\section{Results:}

We present the first use of anchored hybrid enrichment in insect phylogenetics on a dataset containing 30 flower fly species from across all four subfamilies and 11 tribes out of 15 . To produce a phylogenetic hypothesis, 559 loci were sampled to produce a final dataset containing 217,702 sites. We recovered a well resolved topology with bootstrap support values that were almost universally $>95 \%$. The subfamily Eristalinae is recovered as paraphyletic, with the strongest support for this hypothesis to date. The ant predators in the Microdontinae are sister to all other syrphids. Syrphinae and Pipizinae are monophyletic and sister to each other. Larval predation on soft-bodied hemipterans evolved only once in this family. 


\section{Conclusions:}

Anchored hybrid enrichment was successful in producing a robustly supported phylogenetic hypothesis for the syrphids. Subfamilial reconstruction is concordant with recent phylogenetic hypotheses, but with much higher support values. With the newly designed probe kit this analysis could be rapidly expanded with further sampling, opening the door to more comprehensive analyses targeting problem areas in syrphid phylogenetics and ecology.

\section{Introduction}

Thanks in part to modern molecular techniques, the field of biological systematics has made great advances in assembling the Tree of Life. Well-supported phylogenetic hypotheses, based partly or entirely on phylogenomic datasets, now exist for many major animal groups, including (holometabolous) insects (Beutel et al. 2010, Misof et al. 2014), birds (Hackett et al. 2008, McCormack 2013, Prum et al. 2015), mammals (Meredith et al. 2011), and squamates (Pyron et al. 2013). Phylogenomic analyses have been made possible by the dramatically decreasing costs of genome/transcriptome sequencing of non-model organisms (Glenn 2011). However, for many phylogenetic questions, a dense, comprehensive sampling of genomes/transcriptomes is still a prohibitively expensive enterprise. In order to generate these comprehensive phylogenomic data sets, several cost-effective alternatives to whole genome or transcriptome sequencing have been proposed.

One such method is hybrid enrichment (Albert et al. 2007, Gnirke et al. 2009), which uses oligonucleotide probes or "baits" targeting specific areas of the 
genome in question. These probes hybridize to genomic fragments containing the loci of interest, allowing them to be amplified and sequenced using high-throughput sequencing. Originally developed for medical research on human diseases (Gnirke et al. 2009, Bamshad et al. 2011), hybrid enrichment is a flexible technique for which applications in phylogenomic research are just beginning to be realized (Faircloth et al. 2012, Lemmon et al. 2012, McCormack et al. 2013, Eytan et al. 2015). Unlike traditional polymerase chain reaction (PCR), hybrid enrichment techniques can be used to isolate and amplify many loci in a single reaction, and thus greatly improve the representation of single species in terms of gene coverage in phylogenomic analyses. Furthermore, once a probe kit is developed the cost of a project increases primarily by the number of taxa added (unlike Sanger sequencing which increases by the number of taxa and loci added) (Lemmon et al. 2012).

Two major hybrid enrichment methods are currently used for phylogenetic studies: the ultraconserved element (UCE) approach (Faircloth et al. 2012) and anchored hybrid enrichment (AHE) (Lemmon et al. 2012). The UCE approach targets highly conserved noncoding regions of the genome (Faircloth et al. 2012) while AHE targets highly conserved regions primarily in the coding portion of the genome; specifically, it targets these regions flanked by less conserved regions in an attempt to acquire more data useful for phylogenetic inference (Lemmon et al. 2012). AHE probe kits are also designed to target a wide range of taxonomic groups: the initial probe kit was designed for use across all vertebrate taxa. This was accomplished by comparing the complete genome of five model organisms (Lemmon and Lemmon 2013). While recent studies have used the UCE approach to 
study ants (Blaimer et al. 2015) and a related exon-capture method to study brittle stars (Hugall et al. 2015), the present study is the first invertebrate project conducted using the AHE technique, utilizing the first iteration of insect-specific probes to construct a phylogenetic hypothesis of the dipteran family Syrphidae.

Syrphidae is a large and relatively well-known family of Diptera with over 6,200 described species worldwide (Pape and Thompson 2013). The family has traditionally been divided into three subfamilies: Syrphinae, Microdontinae, and Eristalinae (Vockeroth and Thompson 1987). However, Pipizini, a tribe of historically uncertain placement, has recently been elevated to subfamilial level (i.e. Pipizinae) (Mengual et al. 2015). In addition, latest phylogenetic studies using molecular sequence data (Skevington and Yeates 2000) and combined molecular and morphological data (Ståhls et al. 2003) recover Eristalinae as paraphyletic. Finally, the Microdontinae have been alternately placed within what would now be considered Eristalinae (Goffe 1952, Wirth et al. 1965), within Syrphinae (Williston 1886), or as a separate family (Thompson 1969, Thompson 1972, Speight 1987) but are currently considered a subfamily (Hull 1949, Knutson et al. 1975, Thompson et al. 1976, Smith and Vockeroth 1980, Thompson and Vockeroth 1989, Ståhls et al. 2003, Cheng and Thompson 2008, Reemer and Ståhls 2013). In summary, there is no phylogenetic consensus of subfamilial relationships.

The current tribal division of the family is based mostly on adult morphological characters and larval biology (Thompson and Rotheray 1998). A total of 15 tribes are recognized: Microdontini and Spheginobacchini, in Microdontinae; 
Brachyopini, Callicerini, Cerioidini, Eristalini, Merodontini, Milesiini, Rhingiini, Sericomyiini, and Volucellini, in Eristalinae; and Bacchini, Paragini, Syrphini, and Toxomerini, in Syrphinae (Mengual et al. 2015). The subfamily Pipizinae has no tribal subdivision. The classification into tribes has not been generally accepted, and the relationships among them have never been studied in detail for the entire family (Thompson 1972, Thompson 1975, Rotheray and Gilbert 1989, Mengual et al. 2008). Some of the genera have been placed in different tribes and some tribes have even been placed in different subfamilies. For instance, Spheginobacchini has been placed within eristalines, syrphines and microdontines (Ståhls et al. 2003, Rotheray and Gilbert 2011, Reemer and Ståhls 2013) as well as “Pipizini” (Mengual et al. 2015). Moreover, some tribes are not supported by the last molecular phylogenetic studies, such as Brachyopini, Bacchini or Toxomerini, or their placement within a subfamily is uncertain or unresolved as there is no agreement among different works, e.g. Paragini, Volucellini, Merodontini, and Callicerini (Hippa and Ståhls 2005, Mengual et al. 2008, Mengual et al. 2012, Mengual et al. 2015).

Adults of most species of flower flies are conspicuous flower visitors, where they feed on both pollen and nectar (Larson et al. 2001). This behaviour has earned the family the common name "flower flies" (also known as "hoverflies"), and has also generated a large amount of interest in the family as pollinators in both natural ecosystems and agricultural crops (Barrett and Helenurm 1987, Jauker and Wolters 2008, Ssymank et al. 2008, Rader et al. 2009, Zych 2014, Rader et al. 2016). The only exception are the microdontines, whose adults are rarely seen on flowers, and in some species they do not feed at all (Reemer 2014). In contrast to the relatively 
uniform behaviour of the adults, syrphid larvae display an extraordinary diversity of life histories for a single family, including terrestrial and aquatic predators, inquilines in ant, wasp and bumblebee nests, saprophages, mycophages, root borers, stem miners, leaf miners, and wood borers in decaying logs (Heiss 1938, Rotheray 1993, Rotheray and Gilbert 2011). Larvae of Microdontinae are inquilines in ants' nests feeding on eggs, larvae and pupae (Reemer 2013), but also may parasitize ant pupae (Pérez-Lachaud 2014). Immature stages of Eristalinae include saprophages in a wide range of decaying organic media from dung to dead wood, some phytophages in various plants, and some predaceous species, i.e. species of the genus Volucella Geoffrey, 1762 are wasp- and bee-brood predators, and larvae of Nepenthosyrphus Meijere, 1932 are sit-and-wait aquatic predators in the phytotelmata of pitcher plants in SE Asia (Rotheray 1993, Mogi and Chan 1996, Stuke 2000, Rotheray 2003, Morales and Wolff 2010, Rotheray and Gilbert 2011). Larvae of Pipizinae and Syrphinae share a similar feeding mode, but while known pipizine larvae are predatory mostly on woolly or root aphids with waxy secretions and gall-forming hemipterans, the majority of syrphine larvae prey on a broader range of soft-bodied arthropods such as aphids, coccids and psyllids, but also on Thysanoptera, immature Coleoptera, and Lepidoptera (Rojo et al. 2003). The larvae of some Neotropical syrphines develop as stem-borers and leaf-miners in plants or as pollen-feeders (Marin and Comerma 1969, Nishida, Rotheray et al. 2002, Reemer and Rotheray 2009, Weng and Rotheray 2009). This high diversity of natural histories makes syrphid immatures interesting and economically important as they can be biological control agents of plant pests and invasive weeds, re-cyclers of dead 
plant and animal matter, and pests of some ornamental plants (Rotheray 1993, Grosskopf 2005, Grosskopf et al. 2008, Rotheray and Gilbert 2011).

Hence, a robust phylogeny of syrphids is crucial to tackle the evolution of mimicry (Penney et al. 2012), to test the coevolution of microdontines and their ant hosts (Reemer 2013), to infer the evolution of larval life histories and the biology of the common ancestor, and to study the evolution of migratory behaviour.

The aim of the current study was to develop a set of AHE probes for use in Diptera, and to use the newly developed probe set to address the systematic position of the more problematic (e.g. unstable placements, unique morphology) taxa within Syrphidae, especially at the subfamilial and tribal level. Due to their high level of diversity, myriad of larval life histories, historical intractability of a robust subfamilial phylogenetic hypothesis, and economic and ecological significance, Syrphidae are an attractive model organism to test the utility of AHE. The project was accomplished by utilizing AHE to target genomic data from 559 nuclear gene regions (374 used in the final analyses). Although the main goal of this study was to elucidate phylogenetic relationships within the family Syrphidae, sequence data from a total of 12 cyclorrhaphan Diptera families were captured, illustrating the flexibility of the technique.

Although the current study includes all major clades of Syrphidae, the phylogeny proposed here will eventually form the basis for a much larger and more thoroughly sampled phylogenetic study (http://www.canacoll.org/Diptera/Staff/Skevington/Syrphidae/Syrphidae World 
Phylogeny.htm). This initiative is being conducted by a large group of entomologists and promises to be the largest phylogenetic collaboration attempted on a single family of insects.

\section{Methods}

\section{Anchored Hybrid Enrichment Laboratory Data Collection}

Data were collected following the general methods of Lemmon et al. (2012) through the Center for Anchored Phylogenomics at Florida State University (www.anchoredphylogeny.com). Briefly, 50ul of each genomic DNA sample, with quantity ranging from $11.5 \mathrm{ng}-985.3 \mathrm{ng}$ ) was sonicated to a fragment size of $\sim 150$ 350 base pairs (bp) using a Covaris E220 Focused-ultrasonicator with Covaris microTUBES. Subsequently, library preparation and indexing were performed on a Beckman-Coulter Biomek FXp liquid-handling robot following a protocol modified from Meyer and Kirschner (2010) (Meyer and Kircher 2010). One important modification is a size-selection step after blunt-end repair using SPRIselect beads (Beckman-Coulter Inc.; 0.9x ratio of bead to sample volume). Indexed samples were then pooled at equal quantities (typically 12-16 samples per pool), and enrichments were performed on each multi-sample pool using an Agilent Custom SureSelect kit (Agilent Technologies), designed as specified above. After enrichment, the three enrichment pools were pooled in equal quantities for sequencing in one PE150 Illumina HiSeq2000 lane. Sequencing was performed in the Translational Science Laboratory in the College of Medicine at Florida State University. 


\section{Probe Development}

We began with nucleotide alignments of 4485 protein coding genes for 13 insect species identified by Niehuis et al. (2012). Each alignment contained up to 11 members of Holometabola from five orders (Diptera, Hymenoptera, Lepidoptera, Strepsiptera, and Coleoptera) and two non-holometabolous insects (used as outgroup) from two orders (Anoplura and Hemiptera). A full list of the species and their higher taxonomy is given in Table 1 . We then selected a preliminary set of loci containing $>=6$ taxa and at least one consecutive 120 bp region with $>50 \%$ pairwise sequence identity. Sequences for each species were extracted, and exon boundaries were then identified using published genomes (see Table 1 for details) and custom scripts that identified matches between the transcript sequences (Table 2) and the genomes using 40-mers.

Together with the alignments, the exon boundaries were used to identify suitable candidate regions (exons) to target using an Anchored Phylogenomics approach, as described by Lemmon et al. (2012). The following requirements were used to select 962 insect-wide targets: 1) the region was at least 150 bp in length, 2) the region contained no exon boundaries, and 3) the region contained no indels. Details of these targets are given in Supplemental Table 1 (https://bmcevolbiol.biomedcentral.com/articles/10.1186/s12862-016-07140\#MOESM1). Concatenated alignments have been uploaded to the NCBI Sequence Read Archive, with accession numbers available in Table 3. The lengths of these targets ranged from $150 \mathrm{bp}$ to $863 \mathrm{bp}$ (mean=187 bp) whereas the pairwise sequences similarity ranged from $45 \%$ to $84 \%$ (mean=66\%). 
In order to develop an enrichment kit efficient for Diptera, we developed a reference database based on the Drosophila melanogaster sequences contained within the 962 target locus alignments, plus 13 established loci provided by Brian Wiegmann (Wiegmann, Trautwein et al. 2011). The database contained spaced kmers derived from conserved sites within each locus. These were used to scan for homologous loci in seven Diptera genomes and 14 Diptera transcriptomes (see Table 2 for complete list). After the sequence best matching to the references was identified for each species $\mathrm{x}$ locus combination, alignments were estimated for each locus using MAFFT (Katoh and Standley, 2013; v7.023b with -genafpair and maxiterate 1000 flags) (Katoh and Standley 2013). Geneious v5.6.4 (Biomatters, available from http://www.geneious.com) was then used to select well-aligned regions that overlapped with the core insect regions, contained high taxon representation ( $>10$ of 21 lineages), and contained low gaps. The 546 chosen anchor locus alignments contained 121-1497 sites (average of 588 sites) and 48\%-84\% pairwise sequence similarity (average=69\%). The 13 functional locus alignments contained 185-3035 sites (average of 1758 sites) and 50\%-79\% pairwise sequence similarity (average $=66 \%$ ).

Finally, in order to ensure efficient enrichment, we checked for high-copy regions (e.g. microsatellites and transposable elements) in each of the seven genome-derived references as follows. First, a database was constructed for each species using all 15-mers found in the trimmed alignments for that species. We also added to the database all 15-mers that were $1 \mathrm{bp}$ removed from the observed 15mers. The genome for the species was then exhaustively scanned for the presence of 
these 15-mers and matches were tallied at the alignment positions at which the 15mer was found. Alignment regions containing $>100,000$ counts in any of the seven species were masked to prevent probe tiling across these regions. Probes of $120 \mathrm{bp}$ were tiled uniformly at $1.72 x$ tiling density (57681 probes total). Final probe regions and the final probe sequences are available as Supplemental Tables 2 (https://bmcevolbiol.biomedcentral.com/articles/10.1186/s12862-016-07140\#MOESM2) and 3

(https://bmcevolbiol.biomedcentral.com/articles/10.1186/s12862-016-07140\#MOESM3). Scripts used for locus selection and design and alignments are available upon request from ARL.

In essence, the process for choosing probes for the Diptera kit was fundamentally the same as for choosing probes for the vertebrate kit (V1, Lemmon et al. 2012). The only difference was that alignments containing only genomes formed the basis of the vertebrate kit, whereas alignments containing both genomes and transcriptomes formed the basis of the Diptera kit.

\section{Anchored Hybrid Enrichment Bioinformatic Data Analysis} Paired-Read Merging: Typically, between $50 \%$ and $75 \%$ of sequenced library fragments had an insert size between $150 \mathrm{bp}$ and $300 \mathrm{bp}$. Since $150 \mathrm{bp}$ paired-end sequencing was performed, this means that the majority of the paired reads overlap

and thus should be merged prior to assembly. The overlapping reads were identified and merged following Rokyta (Rokyta et al. 2012). In short, for each degree of overlap for each read we computed the probability of obtaining the observed 
number of matches by chance, and selected degree of overlap that produced the lowest probability, with a p-value less than $10^{-10}$ required to merge reads. When reads are merged, mismatches are reconciled using base-specific quality scores, which were combined to form the new quality scores for the merged read (see (Rokyta et al. 2012) for details). Reads failing to meet the probability criterion were kept separate in the assembly. The merging process produces three files one containing merged reads and two containing the unmerged reads.

Assembly: The reads were assembled into contigs using an assembler that makes use of both a divergent reference assembly approach to map reads to the probe regions and a de-novo assembly approach to extend the assembly into the flanks. The reference assembler uses a library of spaced 20-mers derived from the conserved sites of the alignments used during probe design. A preliminary match was called if at least 17 of 20 matches exist between a spaced kmer and the corresponding positions in a read. Reads obtaining a preliminary match were then compared to an appropriate reference sequence used for probe design to determine the maximum number of matches out of 100 consecutive bases (all possible gap-free alignments between the read and the reference were considered). The read was considered mapped to the given locus if at least 55 matches were found. Once a read was mapped, an approximate alignment position was estimated using the position of the spaced 20-mer, and all 60-mers existing in the read were stored in a hash table used by the de-novo assembler. The de-novo assembler identified exact 
matches between a read and one of the 60-mers found in the hash table. Simultaneously using the two levels of assembly described above, the three read files were traversed repeatedly until an entire pass through the reads produced no additional mapped reads.

A list of all 60-mers found in the mapped reads was compiled, the 60-mers were clustered if found together in at least two reads. The 60-mer clusters were then used to separate the reads into clusters for contig estimation. Relative alignment positions of reads within each cluster were then refined in order to increase the agreement across the reads. Up to one gap was also inserted per read if needed to improve the alignment. Note that given sufficient coverage and an absence of contamination, each single-copy locus should produce a single assembly cluster. Low coverage (leading to a break in the assembly), contamination, and gene duplication, can all lead to an increased number of assembly clusters. A whole genome duplication, for example, would increase the number of clusters to two per locus.

Consensus bases were called from assembly clusters as follows. For each site an unambiguous base was called if the bases present were identical or if the polymorphism of that site could be explained as sequencing error, assuming a binomial probability model with the probability of error equal to 0.1 and alpha equal to 0.05 . If the polymorphism could not be explained as sequencing error, the ambiguous base was called that corresponded to the IUPAC code. Called bases were soft-masked (made lowercase) for sites with coverage lower than five. A summary 
of the assembly results is presented in Supplemental Table 4

(https://bmcevolbiol.biomedcentral.com/articles/10.1186/s12862-016-07140\#MOESM4).

Contamination Filtering: In order to filter out possible low-level contaminants, consensus sequences derived from very low coverage assembly clusters ( $<10$ reads) were removed from further analysis. After filtering, consensus sequences were grouped by locus (across individuals) in order to produce sets of homologs.

Orthology: Orthology was determined for each locus as follows. First, a pairwise distance measure was computed for pairs of homologs. To compute the pairwise distance between two sequences, we computed the percent of 20 -mers observed in the two sequences that were found in both sequences. Note that the list of 20-mers was constructed from consecutive 20-mers as well as spaced 20-mers (every third base), in order to allow increased levels of sequence divergence. Using the distance matrix, we clustered the sequences using a Neighbor-Joining algorithm as follows: Pairwise distances were ranked from smallest to largest. Starting with the smallest value, pairs of sequences from the set of homologs (representing the next distance in the list) were joined into the same cluster. If one of the two sequences was already in a cluster, the clusters were merged. Clusters containing homologs originating from the same individual were not joined, such that when clustering was complete, each cluster contained at most one homolog per species. Sequence 
clusters containing fewer than $50 \%$ of the species were removed from downstream processing.

Alignment (MAFFT): Sequences in each orthologous set were aligned using MAFFT v. 7.023b (Katoh and Standley 2013), with --genafpair and --maxiterate 1000 flags.

Alignment Trimming: In order to reduce the error in the data, the alignment for each locus was then trimmed/masked using the following procedure. First, each alignment site was identified as "conserved" if the most common character observed was present in $>40 \%$ of the sequences. This step identified sites for which we were confident were aligned correctly for a sufficient portion of the taxa (typically third codon potions would not be included here). Second, $20 \mathrm{bp}$ regions of each sequence that contained $<10$ stable sites were masked. This step identified regions of each sequence that were not well aligned to the majority of the sequences and thus should be masked. Third, sites with fewer than 12 unmasked bases were removed from the alignment. This step identified large regions of the alignments that should be removed entirely from the alignment because they contain large quantities of missing data (Lemmon and Lemmon 2008).

\section{Taxon sampling}

Representatives of all four Syrphidae subfamilies and 11 tribes were analysed. We also included taxa of another four dipteran families, i.e. Platypezidae [Platypeza sp.], 
Pipunculidae [Chalarus spurius (Fallén, 1816) and Pipunculus sp.0N12], Sepsidae [Themira nigricornis (Meigen, 1826)], and Tachinidae [Epalpus signifer (Walker, 1849)]. A total of 30 flower fly species were sampled (Table 3). Syrphid taxa come from four different Biogeographical Regions, but the majority are Nearctic specimens. Morphological identification of syrphids and pipunculids were provided by A.D.Y and J.H.S., other outgroup taxa were morphologically identified by colleagues at the Canadian National Collection of Insects, Arachnids, and Nematodes (CNC).

\section{DNA Extraction}

Genomic DNA extractions were obtained with the QIAGEN DNeasy kit (Qiagen Inc., Santa Clara, CA, USA). Full specimens were extracted overnight at $56^{\circ} \mathrm{C}$, and total DNA was purified the following day following the manufacturer's protocol. Following extraction, specimens were critical-point dried with the EM CPD300 (Leica Microsystems, Vienna, Austria) and deposited at CNC.

\section{Vouchers}

Specimens for the study were collected by Malaise trap or hand-collecting, preserved in $95-100 \%$ ethanol, and placed in a $-80^{\circ} \mathrm{C}$ freezer until extraction. The voucher data and unique identifiers for the specimens used for the molecular study are presented in Table 3. Specimens have since been critical point dried, mounted, labeled and deposited in the Canadian National Collection of Insects, Arachnids and Nematodes. 
The 5' region of the mitochondrial Cytochrome $c$ Oxidase Subunit I (COI) gene was sequenced for each specimen in order to act as a surrogate voucher and allow linkage of the exemplars to a large molecular dataset being assembled. Amplification, purification, sequencing and contig assembly were carried out as described in Gibson et al. (2010).

COI sequence alignment was straightforward as no indels (insertions or deletions) were found. The alignment was made by hand using Mesquite v. 2.74 (Maddison and Maddison 2010) and translated into amino acids to ensure that there were no stop codons. Sequences were submitted to BOLD and uploaded from there to GenBank (Table 3).

\section{Phylogeny Estimation}

A maximum likelihood (ML) tree (with 100 bootstrap replicates) for a single concatenated matrix was estimated using RAxML v. 7.2.6 (Stamatakis 2006), with the GTR + G substitution model partitioned by locus under default parameters. Platypeza was used to root the tree.

\section{Results}

Trimmed alignments contained 35 taxa and 217,702 sites (across 343 chosen loci), of which 89,534 sites were informative. The concatenated dataset was largely complete, with only 6\% missing data. Maximum Likelihood estimation (Fig.1) of the present concatenated dataset produced a fully resolved tree, with 31/32 nodes 
(97\%) supported by $>95 \%$ bootstrap support (BS) values. As expected, Syrphidae was recovered as a monophyletic group with Microdon Meigen, 1803 as the sister to other lineages $(\mathrm{BS}=100 \%)$. The sister clade to the Syrphidae included Pipunculidae + Schizophora. The subfamilies Pipizinae and Syrphinae were resolved as clades. The potential monophyly of the subfamily Microdontinae could not be established (only one taxon included) and Eristalinae was resolved as non-monophyletic. A paraphyletic Eristalinae was placed sister to Syrphinae + Pipizinae. Within the eristalines, several tribes were resolved monophyletic based on the studied taxa. Merodontini (Eumerus Meigen, 1822 + Merodon Meigen, 1803) was recovered as a clade sister to the remainder of the Eristalinae. Volucellini (Graptomyza Weidemann, 1820 + Copestylum Macquart, 1846), Rhingiini (Rhingia Scopoli, 1763 + Cheilosia Meigen, $1822+$ Ferdinandea Rondani, 1844) and Milesiini (Brachypalpus Macquart, $1834+$ Xylota Meigen, 1822) were also found to be monophyletic. The three remaining tribes that were included in the analysis (Eristalini, Brachyopini, and Callicerini) only had a single member included, so potential monophyly could not be established. Within Syrphinae, three of the four tribes were included, i.e. Syrphini, Bacchini, and Paragini, but not Toxomerini. Bacchini was recovered as paraphyletic, with Melanostoma Schiner, 1860 placed as sister to the remainder of the Syrphinae, and a clade consisting of Baccha Fabricius, $1805+$ Platycheirus Lepeletier \& Serville, 1828 sister to Syrphinae excluding Melanostoma. Syrphini is a large tribe comprised of the majority of the syrphine genera, and formed a single clade with Paragus (the sole member of Paragini) resolved within it. 


\section{Discussion}

This analysis represents the first iteration of newly developed Diptera probes for AHE. While the probes were developed by analysing the genome of only 21 insect species, they were successfully used to extract sequence data from 18 additional Dipteran families (data not shown). Furthermore, while 559 loci were targeted designed, only 343 loci were included in the final analysis in order to minimize missing data. As more invertebrate genomes become available and probe kits are refined, ever larger datasets will be attainable from a broad spectrum of invertebrate taxa for a fraction of the cost of traditional Sanger sequencing methods (Lemmon et al. 2012).

The ML analysis produced a fully-resolved phylogram, with only one node with low bootstrap support (BS = 68\%) (see Fig. 1). While previous analyses have recovered similar phylogenies (Skevington and Yeates 2000, Ståhls et al. 2003, Mengual et al. 2015), no previous works have recovered a fully-resolved tree with high support. A possible explanation for this surprising result is the high number of loci and bp included in our analysis bases on the newly-designed probes, which might allow fully resolved phylogenies for other dipteran families to be produced. The present analysis includes the largest genomic dataset ever created for the phylogenetic analysis of an insect/Diptera family, with 343 loci and 217,702 bp.

The two Pipunculidae taxa were recovered as sister to Epalpus signifer (Tachinidae) and Themira nigricornis (Sepsidae), both schizophoran flies. Although traditional morphological analyses (Hennig 1966, Griffiths 1972, McAlpine 1989, Rotheray and Gilbert 2008) have supported a sister group relationship between 
Syrphoidea (Pipunculidae + Syrphidae) and Schizophora, more recent morphological (Tachi 2014) and molecular (Wiegmann et al. 2011) analyses suggest a sister group relationship between Pipunculidae and Schizophora, rendering Syrphoidea paraphyletic.

Placement of Microdontinae has a chequered history as pointed out in the introduction. The "ant flies" are morphologically very distinct from the remaining Syrphidae and all species with known larval histories are associated with ants. Larvae are either predatory or parasitoids in ant nests and have developed elaborate pheromone mimicry to carry out this feat (Howard et al. 1990, Howard et al. 1990, Rotheray and Gilbert 2011, Pérez-Lachaud 2014). Strong morphological and ecological specializations within the group have made microdontines very difficult to place into phylogenetic context. Thompson (1969) was the first to provide quantitative evidence that they are sister to all other Syrphidae species (based on adult morphology). Despite this, other contradictory hypotheses have continued to be proposed. For example, in their study of larval characters and evolution, Rotheray and Gilbert (1999) presented a hypothesis supporting a sistergroup relationship between Microdontinae and pipizines and syrphines. This hypothesis assumed a single predatory larval lineage within Syrphidae. Our study refutes this and supports Thompson (1969) and several recent molecular studies using Sanger sequence data (Skevington and Yeates 2000, Ståhls et al. 2003, Reemer and Ståhls 2013, Mengual et al. 2015). Proposals as per Thompson (1972) and Speight $(1987,2014)$ have been made to elevate the ant flies to family status and although our present results do not refute this, it remains an argument largely based 
on the perceived level of morphological and ecological difference of ant flies from other syrphids. Microdontinae is a highly diverse clade and still understudied taxonomically and biologically (Reemer 2013, Reemer and Ståhls 2013, Reemer and Ståhls 2013, Pérez-Lachaud 2014). Only one species was available for the present study, but the inclusion of members of the Spheginobacchini as well as other taxa not closely related to the genus Microdon (Reemer and Ståhls 2013) will allow us to test the relationships among the taxa of this subfamily.

Eristalinae was recovered as paraphyletic in the present study. The monophyly of Eristalinae is supported by several studies and the currently followed classification follows this line of reasoning (Goffe 1952, Hartley 1961, Vockeroth 1992, Thompson and Rotheray 1998). In contrast, evidence from more recent surveys using adult morphological and/or molecular characters, with a very limited number of loci, resolve Eristalinae as paraphyletic (Skevington and Yeates 2000, Ståhls et al. 2003, Hippa and Ståhls 2005, Mengual et al. 2015). Our analysis is the first to use AHE data from hundreds of loci, and ML analysis of the data provides support for a non-monophyletic Eristalinae (Fig. 1). In the present study, Merodontini was resolved as sister group of the other eristalines + syrphines + pipizines, and Volucellini and Helophilus Meigen, 1822 (Eristalini) were recovered in different nodes, with the other included eristaline tribes forming a clade, i.e. Rhingiini, Sphegina Meigen, 1822 (Brachyopini), Callicerini and Milesiini. Our taxon sampling is not enough to make conclusions about the tribal relationships within this subfamily. Consequently, a larger and broader taxon sampling is still required, including tribes that were not available for the present study such as Cerioidini and 
Sericomyiini, to understand how eristaline tribes are related. The only weakly supported node on the maximum likelihood tree is within the Eristalinae.

Eristalinae is the subfamily with the highest number of species and larval biology diversity, and it is reflected in the classification with the recognition of nine tribes and several subtribes. Addition of more taxa in future studies will address the question of the monophyly of the subfamily and the tribes, and will also help to better understand larval evolution within this incredibly diverse group of flies.

Syrphinae and Pipizinae were reciprocally monophyletic and sister groups to each other. The placement of Pipizinae as sister to Syrphinae is a phylogenetic hypothesis that has gained increasing support in recent years, and last phylogenetic works have recovered Pipizinae either within Syrphinae (Cheng et al. 2000, Mengual, Ståhls et al. 2008), or sister to it (Rotheray and Gilbert 1999, Skevington and Yeates 2000, Ståhls, Hippa et al. 2003, Mengual et al. 2015). The frequent placement of Pipizinae within Eristalinae owes much to the fact that many early classification schemes were based largely or entirely on adult morphological characters. The present results strongly suggest a common origin of these two groups, which implies that predatory larvae feeding on soft-bodied arthropods have evolved only once in the evolution of the Syrphidae, and they corroborate previous surveys and the recent elevation of Pipizinae to subfamilial level (Mengual et al. 2015). Future studies will explore the interrelationships of the members of this subfamily and will test the hypothesis exposed by Vujić et al. (2013). 
Finally, the resolution of Syrphinae as a monophyletic group was not unexpected as virtually all existing flower fly phylogenies hypothesize that Syrphinae is a clade. In contrast, the current tribal classification within Syrphinae is not supported in our analyses in concordance with the last phylogenetic studies (Rotheray and Gilbert 1999, Ståhls et al. 2003, Mengual et al. 2008, Mengual et al. 2015, Miranda 2016). Bacchini was found to be paraphyletic, and its members (Melanostoma, Platycheirus and Baccha) were resolved in two groups, partly in agreement with previous studies (Ståhls et al. 2003, Mengual et al. 2008, Mengual et al. 2015). Paragini, a syrphine tribe of historically uncertain placement, was resolved as sister to Scaeva Fabricius, 1805 + Betasyrphus Matsumura, 1917, making the current tribe Syrphini paraphyletic. Our results corroborate the hypothesis by Rotheray \& Gilbert (1989), using larval morphological characters, and by Mengual (2015) and (Mengual, Ståhls et al. 2015), using molecular data alone or in combination with adult morphological characters respectively. Addition of more taxa and the inclusion of the tribe Toxomerini will help to understand the tribal classification of Syrphinae, to define new tribal groups, and, the most important, to study the evolution of predation within this group to answer why and how some taxa became phytophagous secondarily.

The scenario recovered in the present analysis using AHE data shows that predation evolved at least three times in different groups with distinct feeding strategies, viz. Pipizinae + Syrphinae, Microdontinae and Volucellini (although the genus Volucella was not studied). A key piece into this puzzle is the unknown biology of the immatures of Spheginobacchini, which would help to understand the 
relation between microdontines and the rest of flower flies. Excellent mimics of wasps and bumblebees appear in several groups, especially within Eristalinae in genera like Temnostoma Lepeletier and Serville, 1828, Spilomyia Meigen, 1803 or Volucella. The existence of a broad spectrum from non-mimics, through partial or imperfect mimics, to perfect mimics might indicate a multiple origin for mimicry. The same scenario is found when migratory species are taken into consideration based in our results. Species like Episyrphus balteatus (De Geer, 1776), Sphaerophoria scripta (Linnaeus, 1758) or members of Scaeva, Platycheirus and Helophilus are well-known migrants but little has been studied about the characteristics, origin and mechanisms of these migrations. A fully resolved exhaustively sampled phylogeny based on AHE has the potential to resolve these questions.

\section{Conclusions}

This is the first time that AHE technique is used on an extended and very diverse group of insects and represents the largest dataset assembled to bear on the phylogeny of a dipteran group. The price and repeatability using the present probe kit makes this technique a reliable methodology for future research using large output sequence datasets. Present results corroborate a number of earlier findings and hypotheses, although this dataset should be considered preliminary due to the small taxon sample. 
The next step, that is building upon a framework with more thorough taxon sampling of the many morphologically highly diverse groups, will create the most comprehensive hypothesis ever made for a large lineage of flies. With such a high level of ecological and morphological diversity, a detailed phylogeny of Syrphidae will support future work in fields such as pollination biology and biological control, and will help to answer major challenging questions that remain open, such as the evolution of inquiline-host associations in myrmecophilic flies, the evolution of larval feeding behaviour, the development of perfect and imperfect mimicry, the origin and biogeography of the different taxon groups, as well as the patterns of migratory behaviour. As it stands, this study provides a test for previous phylogenetic work on syrphids and illustrates that anchored hybrid enrichment is a useful technique for rapidly assembling comprehensive, large datasets for phylogenetic hypothesis testing. Current anchored data collection and analysis pipelines allow 96 samples to be processed in as little as three weeks, from DNA extracts to trimmed alignments and preliminary phylogeny estimates (www.anchoredphylogeny.com).

\section{Funding:}

This study was supported by funding to JHS from Agriculture and Agri-Food Canada, and the Natural Sciences and Engineering Research Council of Canada, to ARL and EML by NSF IIP1313554 and to BMW by NSF DEB1257960. 
Acknowledgements We are grateful for lab assistance provided by Brian K. Cassel, Dept. of Entomology, NC State University. The project could not have been completed without the support of transcriptomes supplied by 1 KITE.

\section{References}

Albert, T. J., M. N. Molla, D. M. Muzny, L. Nazareth, D. Wheeler, X. Song, T. A. Richmond, C. M. Middle, M. J. Rodesch and C. J. Packard (2007). "Direct selection of human genomic loci by microarray hybridization." Nat Methods 4(11): 903-905.

Bamshad, M. J., S. B. Ng, A. W. Bigham, H. K. Tabor, M. J. Emond, D. A. Nickerson and J. Shendure (2011). "Exome sequencing as a tool for Mendelian disease gene discovery." Nat Rev Genet 12(11): 745-755.

Barrett, S. C. and K. Helenurm (1987). "The reproductive biology of boreal forest herbs. I. Breeding systems and pollination." Can I Bot 65(10): 2036-2046.

Beutel, R. G., F. Friedrich, T. Hörnschemeyer, H. Pohl, F. Hünefeld, F. Beckmann, R. Meier, B. Misof, M. F. Whiting and L. Vilhelmsen (2010). "Morphological and molecular evidence converge upon a robust phylogeny of the megadiverse Holometabola." Cladistics 26: 1-15.

Blaimer, B. B., S. G. Brady, T. R. Schultz, M. W. Lloyd, B. L. Fisher and P. S. Ward (2015). "Phylogenomic methods outperform traditional multi-locus approaches in resolving deep evolutionary history: a case study of formicine ants." BMC evolutionary biology 15(1): 1. 
Cheng, X.-Y. and F. C. Thompson (2008). "A generic conspectus of the Microdontinae (Diptera: Syrphidae) with the description of two new genera from Africa and China." Zootaxa 1879: 21-48.

Cheng, X. Y., J. Lu, C. M. Huang, H. Z. Zhou, Z. H. Dai and G. X. Zhang (2000).

"Determination of phylogenetic position of Pipizini (Diptera : Syrphidae): based on molecular biological and morphological data." $\underline{\text { Sci China Ser C }}$ 43(2): 146-156.

Eytan, R. I., B. R. Evans, A. Dornburg, A. R. Lemmon, E. M. Lemmon, P. C. Wainwright and T. J. Near (2015). "Are 100 enough? Inferring acanthomorph teleost phylogeny using Anchored Hybrid Enrichment." BMC Evol Biol 15(1): 1.

Faircloth, B. C., J. E. McCormack, N. G. Crawford, M. G. Harvey, R. T. Brumfield and T. C. Glenn (2012). "Ultraconserved elements anchor thousands of genetic markers spanning multiple evolutionary timescales." Syst Biol: sys004.

Gibson, J. F., J. H. Skevington and S. Kelso (2010). "Placement of the Conopidae (Diptera) within the Schizophora based on ten mtDNA and nrDNA gene regions." Mol Phylogen Evol 56: 91-103.

Glenn, T. C. (2011). "Field guide to next-generation DNA sequencers." $\underline{\text { Mol Ecol }}$ Resour 11(5): 759-769.

Gnirke, A., A. Melnikov, J. Maguire, P. Rogov, E. M. LeProust, W. Brockman, T. Fennell, G. Giannoukos, S. Fisher and C. Russ (2009). "Solution hybrid selection with ultra-long oligonucleotides for massively parallel targeted sequencing." $\underline{\mathrm{Nat}}$ Biotechnol 27(2): 182-189. 
Goffe, C. E. R. (1952). "An outline of a revised classification of the Syrphidae (Diptera) on phylogenetic lines." Trans Soc Br Entomol 11(4): 97-124. Griffiths, G. C. D. (1972). The phylogenetic classification of Diptera Cyclorrhapha with special reference to the structure of the male postabdomen. The Hague, Dr. W. Junk N. V.

Grosskopf, G. (2005). "Biology and life history of Cheilosia urbana (Meigen) and Cheilosia psilophthalma (Becker), two sympatric hoverflies approved for the biological control of hawkweeds (Hieracium spp.) in New Zealand." Biol Control 35: 142-154.

Grosskopf, G., L. Wilson and J. Littlefield (2008). Host-range investigations of potential biological control agents of alien invasive hawkweeds (Hieracium spp.) in the USA and Canada: an overview. Proceedings of the XII International Symposium on Biological Control of Weeds. Edited by MH Julien, R. Sforza, MC Bon, CH Evans, PE Hatcher, HL Hinz, and BG Rector. CAB International, Wallingford, UK, Citeseer.

Hackett, S. J., R. T. Kimball, S. Reddy, R. C. Bowie, E. L. Braun, M. J. Braun, J. L. Chojnowski, W. A. Cox, K.-L. Han and J. Harshman (2008). "A phylogenomic study of birds reveals their evolutionary history." Science 320(5884): 17631768.

Hartley, J. C. (1961). "A Taxonomic account of the larvae of some British Syrphidae." Proc Zool Soc Lond (Reprinted) 136(Part 4): 505-573. 
Heiss, E. M. (1938). A Classification of the larvae and puparia of the Syrphidae of Illinois exclusive of aquatic forms. Urbana, Illinois, The University of Illinois Press.

Hennig, W. (1966). Phylogenetic Systematics. Champaign, IL., University of Illinois Press.

Hippa, H. and G. Ståhls (2005). "Morphological characters of adult Syrphidae: descriptions and phylogenetic utility." Acta Zool Fenn 215: 1-72.

Howard, R. W., R. D. Akre and W. B. Garnett (1990). "Chemical mimicry in an obligate predator of carpenter ants (Hymenoptera: Formicidae)." $\underline{\text { Ann }}$ Entomol Soc Am 83(3): 607-616.

Howard, R. W., D. W. Stanley Samuelson and R. D. Akre (1990). "Biosynthesis and chemical mimicry of cuticular hydrocarbons from the obligate predator, Microdon albicomatus Novak (Diptera: Syrphidae) and its ant prey, Myrmica incompleta Provancher (Hymenoptera: Formicidae)." \Kans Entomol Soc 63(3): 437-443.

Hugall, A. F., T. D. O'Hara, S. Hunjan, R. Nilsen and A. Moussalli (2015). "An exoncapture system for the entire class Ophiuroidea." Molecular biology and evolution: msv216.

Hull, F. M. (1949). "The morphology and inter-relationship of the genera of syrphid flies, recent and fossil." Tran Zool Soc Lond 26: 257-408.

Jauker, F. and V. Wolters (2008). "Hover flies are efficient pollinators of oilseed rape." Oecologia 156(4): 819-823. 
Katoh, K. and D. M. Standley (2013). "MAFFT multiple sequence alignment software version 7: improvements in performance and usability." Mol Biol Evol 30(4): 772-780.

Knutson, L. V., F. C. Thompson and J. R. Vockeroth (1975). Family Syrphidae. $\underline{A}$ Catalog of the Diptera of the Oriental Region. Volume 2. Suborder Brachycera Through Division Aschiza, Suborder Cyclorrhapha. M. D. Delfinado and D. E. Hardy. Honolulu, HI, The University Press of Hawaii. 2: 307-374.

Larson, B. M., P. G. Kevan and D. W. Inouye (2001). "Flies and flowers: taxonomic diversity of anthophiles and pollinators." Can Entomol 133(4): 439-466.

Lemmon, A. R., S. A. Emme and E. M. Lemmon (2012). "Anchored hybrid enrichment for massively high-throughput phylogenomics." Syst Biol 61(5): 727-744.

Lemmon, A. R. and E. M. Lemmon (2008). "A likelihood framework for estimating phylogeographic history on a continuous landscape." Syst Biol 57(4): 544561.

Lemmon, E. M. and A. R. Lemmon (2013). "High-throughput genomic data in systematics and phylogenetics." Annu Rev Ecol Evol Syst 44: 99-121.

Maddison, W. P. and D. R. Maddison (2010). Mesquite: a modular system for evolutionary analysis.

Marin, A. and J. Comerma (1969). "Nota sobre las larvas de Mesograpta polita (Say)(Syrphidae, Diptera) en espigas de maíz (Zea mays L.) en El Limón, Aragua, Venezuela [Note on the larvae of Mesograpta polita (Say)(Syrphidae, Diptera) on the staminate flowers of corn (Zea mays L.) in El Limón, Aragua, Venezuela]." Agronomía tropical 19: 335-339. 
McAlpine, J. F. (1989). Phylogeny and classification of the Muscomorpha. Manual of Nearctic Diptera. J. F. McAlpine. Ottawa, Agriculture Canada Monograph No. 32, Research Branch, Agriculture Canada. 3: 1397-1505.

McCormack, J. E., M. G. Harvey, B. C. Faircloth, N. G. Crawford, T. C. Glenn and R. T. Brumfield (2013). "A phylogeny of birds based on over 1,500 loci collected by target enrichment and high-throughput sequencing." PLoS One 8(1): e54848. Mengual, X. (2015). "The systematic position and phylogenetic relationships of Asiobaccha Violovitsh (Diptera, Syrphidae)." \Asia-Pacif Entomol 18: 397 408.

Mengual, X., G. Ståhls and S. Rojo (2008). "First phylogeny of predatory flower flies (Diptera, Syrphidae, Syrphinae) using mitochondrial COI and nuclear 28S rRNA genes: conflict and congruence with the concurrent tribal classification." Cladistics 24: 543-562.

Mengual, X., G. Ståhls and S. Rojo (2012). "Is the mega-diverse genus Ocyptamus (Diptera, Syrphidae) monophyletic? Evidence from molecular characters including the secondary structure of 28S rRNA." Mol Phylogen Evol 62: 191205.

Mengual, X., G. Ståhls and S. Rojo (2015). "Phylogenetic relationships and taxonomic ranking of pipizine flower flies (Diptera: Syrphidae) with implications for the evolution of aphidophagy." Cladistics: 1-18.

Meredith, R. W., J. E. Janečka, J. Gatesy, O. A. Ryder, C. A. Fisher, E. C. Teeling, A. Goodbla, E. Eizirik, T. L. Simão and T. Stadler (2011). "Impacts of the 
Cretaceous Terrestrial Revolution and KPg extinction on mammal diversification." Science 334(6055): 521-524.

Meyer, M. and M. Kircher (2010). "Illumina sequencing library preparation for highly multiplexed target capture and sequencing." Cold Spring Harb Protoc 2010(6): t5448.

Miranda, G. F. G., Skevington, J. H., Marshall, S. A., Kelso, S. (2016). "The genus Ocyptamus Macquart (Diptera: Syrphidae): a molecular phylogenetic analysis (in review)."

Misof, B., S. Liu, K. Meusemann, R. S. Peters, A. Donath, C. Mayer, P. B. Frandsen, J. Ware, T. Flouri and R. G. Beutel (2014). "Phylogenomics resolves the timing

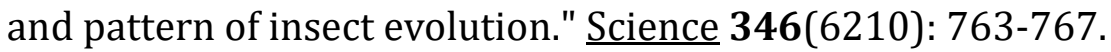

Mogi, M. and K. L. Chan (1996). "Predatory habits of dipteran larvae inhabiting Nepenthes pitchers." Raffles Bull Zool 44(1): 233-245.

Morales, G. E. and M. Wolff (2010). "Insects associated with the composting process of solid urban waste separated at the source." Rev Bras Entomol 54(4): 645653.

Niehuis, O., G. Hartig, S. Grath, H. Pohl, J. Lehmann, H. Tafer, A. Donath, V. Krauss, C. Eisenhardt, J. Hertel, M. Petersen, C. Mayer, K. Meusemann, Ralph S. Peters, Peter F. Stadler, Rolf G. Beutel, E. Bornberg-Bauer, Duane D. McKenna and B. Misof (2012). "Genomic and Morphological Evidence Converge to Resolve the Enigma of Strepsiptera." Curr Biol 22(14): 1309-1313. 
Nishida, K., G. Rotheray and F. C. Thompson (2002). "First non-predaceous syrphine flower fly (Diptera: Syrphidae): A new leaf-mining Allograpta from Costa Rica." Stud Dipterol 9(2): 421-436.

Pape, T. and F. C. Thompson. (2013, 13 June 2013). "Systema Dipterorum. The Biosystematic Database of World Diptera. Version 1.5." 1.5. Retrieved 30 April 2015, 2015, from http://www.diptera.org/.

Penney, H. D., C. Hassall, J. H. Skevington, K. R. Abbott and T. N. Sherratt (2012). "A comparative analysis of the evolution of imperfect mimicry." Nature 483 : 461-466.

Pérez-Lachaud, G. J., M.A.; Reemer, M.; Lachaud, J.-P. (2014). "An unusual, but not unexpected, evolutionary step taken by syrphid flies: the first record of true primary parasitoidism of ants by Microdontinae." Biol J Linn Soc 111: 462472.

Prum, R. O., J. S. Berv, A. Dornburg, D. J. Field, J. P. Townsend, E. M. Lemmon and A. R. Lemmon (2015). "A comprehensive phylogeny of birds (Aves) using targeted next-generation DNA sequencing." Nature 526: 569-573.

Pyron, R. A., F. T. Burbrink and J. J. Wiens (2013). "A phylogeny and revised classification of Squamata, including 4161 species of lizards and snakes." BMC Evol Biol 13(1): 93.

Rader, R., I. Bartomeus, L. A. Garibaldi, M. P. Garratt, B. G. Howlett, R. Winfree, S. A. Cunningham, M. M. Mayfield, A. D. Arthur and G. K. Andersson (2016). "Nonbee insects are important contributors to global crop pollination." Proc Natl Acad Sci 113(1): 146-151. 
Rader, R., B. G. Howlett, S. A. Cunningham, D. A. Westcott, L. E. Newstrom-Lloyd, M. K. Walker, D. A. Teulon and W. Edwards (2009). "Alternative pollinator taxa are equally efficient but not as effective as the honeybee in a mass flowering crop." \ournal of Applied Ecology 46(5): 1080-1087.

Reemer, M. (2013). "Review and phylogenetic evaluation of associations between Microdontinae (Diptera: Syrphidae) and ants (Hymenoptera: Formicidae)." Psyche 2013: 1-9.

Reemer, M. (2014). "A review of Microdontinae (Diptera: Syrphidae) of Surinam, with a key to the Neotropical genera." Tijdschr Entomol 157: 27-57.

Reemer, M. and G. E. Rotheray (2009). "Pollen feeding larvae in the presumed predatory syrphine genus Toxomerus Macquart (Diptera, Syrphidae)." L Nat Hist 43(15): 939-949.

Reemer, M. and G. Ståhls (2013). "Generic revision and species classification of the Microdontinae (Diptera, Syrphidae)." ZooKeys 288: 1-213.

Reemer, M. and G. Ståhls (2013). "Phylogenetic relationships of Microdontinae (Diptera: Syrphidae) based on molecular and morphological characters." Syst Entomol 38: 661-688.

Rojo, S., F. S. Gilbert, M. A. Marcos-Garcia, J. M. Nieto and M. P. Mier (2003). A World Review of Predatory Hoverflies (Diptera, Syrhidae: Syrphinae) and Their Prey. Alicante, Spain, CIBIO Ediciones.

Rokyta, D. R., A. R. Lemmon, M. J. Margres and K. Aronow (2012). "The venom-gland transcriptome of the eastern diamondback rattlesnake (Crotalus adamanteus)." BMC genomics 13(1): 1. 
Rotheray, G. (2003). "The predatory larvae of two Nepenthosyrphus species living in pitcher plants (Diptera, Syrphidae)." Stud Dipterol 10: 219-226.

Rotheray, G. and F. Gilbert (1999). "Phylogeny of Palaearctic Syrphidae (Diptera): evidence from larval stages." Zool J Linn Soc 127(1): 1-112.

Rotheray, G. and F. Gilbert (2011). The Natural History of Hoverflies. Cardigan, UK, Forrest Text.

Rotheray, G. E. (1993). Colour Guide to Hoverfly Larvae (Diptera, Syrphidae). Sheffield, England, Derek Whiteley.

Rotheray, G. E. and F. Gilbert (2008). "Phylogenetic relationships and the larval head of the lower Cyclorrhapha (Diptera)." Zool J Linn Soc 153: 287-323.

Rotheray, G. E. and F. S. Gilbert (1989). "The phylogeny and systematics of European predacious Syrphidae (Diptera) based on larval and puparial stages." Zoo I Linnaean Soc 95(1): 29-70.

Skevington, J. H. and D. K. Yeates (2000). "Phylogeny of the Syrphoidea (Diptera) inferred from mtDNA sequences and morphology with particular reference to classification of the Pipunculidae (Diptera)." Mol Phylogen Evol 16(2): 212-224.

Smith, K. G. V. and J. R. Vockeroth (1980). 38. Family Syrphidae. Catalogue of the Diptera of the Afrotropical Region. R. W. Crosskey. London, Publications of the British Museum (Natural History): 488-510.

Speight, M. C. D. (1987). "External Morphology of Adult Syrphidae (Diptera)." Tijdschrift voor Entomologie 130: 141-175. 
Speight, M. C. D. (2014). StN key for the identification of the genera of European Syrphidae (Diptera). Syrph the Net, the database of European Syrphidae. Dublin, Syrph the Net publications.

Ssymank, A., C. A. Kearns, T. Pape and F. C. Thompson (2008). "Pollinating flies (Diptera): a major contribution to plant diversity and agricultureal production." Biodiversity 9(1-2): 86-89.

Ståhls, G., H. Hippa, G. Rotheray, J. Muona and F. Gilbert (2003). "Phylogeny of Syrphidae (Diptera) inferred from combined analysis of molecular and morphological characters." Syst Entomol 28(4): 433-450.

Stamatakis, A. (2006). "RAxML-VI-HPC: maximum likelihood-based phylogenetic analyses with thousands of taxa and mixed models." Bioinformatics 22(21): 2688-2690.

Stuke, J.-H. (2000). "Phylogenetische Rekonstruktion der Verwandtschaftsbeziehungen innerhalb der Gattung Cheilosia Meigen, 1822 anhand der Larvenstadien (Diptera: Syrphidae). [Phylogenetic relationships within the genus Cheilosia Meigen, 1822, as evidenced by the larval stages (Diptera: Syrphidae)] " Stud Dipterol Supplement 8: 1-118.

Tachi, T. (2014). "Homology of the metapleuron of Cyclorrhapha, with discussion of the paraphyly of Syrphoidea (Diptera: Aschiza)." Insect Syst Evol: 1-20.

Thompson, F. C. (1969). "A new genus of Microdontine flies (Diptera: Syrphidae) with notes on the placement of the subfamily." Psyche 76: 74-85.

Thompson, F. C. (1972). "A contribution to a generic revision of the Neotropical Milesinae (Diptera: Syrphidae)." Arquivos de Zoologica 23(2): 73-215. 
Thompson, F. C. (1975). "Notes on the status and relationships of some genera in the tribe Milesiini (Diptera: Syrphidae)." Proc Entomol Soc Wash 77(3): 291-305.

Thompson, F. C. and G. Rotheray (1998). Family Syrphidae. Contributions to a manual of Palaearctic Diptera (with species reference to flies of economic importance). L. Papp and B. Darvas. Budapest, Science Herald. 3, Higher Brachycera: 81-139.

Thompson, F. C. and J. R. Vockeroth (1989). "51. Family Syrphidae." Bishop Museum Special Publication 86: 437-458.

Thompson, F. C., J. R. Vockeroth and Y. S. Sedman (1976). 46. Family Syrphidae. $\underline{A}$ Catalogue of the Diptera of the Americas South of the United States. São Paulo, Museu de Zoologia, Universidade de São Paulo. Part 3. Cyclorrhapha: $1-195$.

Vockeroth, J. R. (1992). The flower flies of the subfamily Syrphinae of Canada, Alaska and Greenland. Ottawa, Canada Communications Group - Publishing.

Vockeroth, J. R. and F. C. Thompson (1987). Syrphidae. Manual of Nearctic Diptera. J. F. McAlpine, B. V. Peterson, G. E. Shewell et al. Ottawa, Canadian Government Publishing Centre. 2: 713-743.

Vujić, A. S., G.; Ačanski, J.; Bartsch, H.; Bygebjerg, R.; Stefanović, A. (2013). "Systematics of Pipizini and taxonomy of European Pipiza Fallén: molecular and morphological evidence (Diptera, Syrphidae)." Zool Scr 42(3): 288-305.

Weng, J. and G. Rotheray (2009). "Another non-predaceous syrphine flower fly (Diptera: Syrphidae): pollen feeding in the larva of Allograpta micrura." $\underline{\text { Stud }}$ Dipterol 15(2008): 245-258. 
Wiegmann, B. M., M. D. Trautwein, I. S. Winkler, N. B. Barr, J.-W. Kim, C. Lambkin, M. A. Bertone, B. K. Cassel, K. M. Bayless, A. M. Heimberg, B. M. Wheeler, K. J. Peterson, T. Pape, B. J. Sinclair, J. H. Skevington, V. Blagoderov, J. Caravask, S. N. Kutty, U. Schmidt-Ott, G. E. Kampmeier, F. C. Thompson, D. A. Grimaldi, A. T. Beckenbach, G. W. Courtney, M. Friedrich, R. Meier and D. K. Yeates (2011). "Episodic radiations in the fly tree of life." Proc Natl Acad Sci U S A 108(14): $5690-5695$.

Williston, S. W. (1886). "Synopsis of the North American Syrphidae." Bulletin of the United States National Museum 31: i-xxx, 1-335.

Wirth, W. W., Y. S. Sedman and H. V. J. Weems (1965). Family Syrphidae. A catalog of the Diptera of America north of Mexico. A. Stone, C. W. Sabrosky, W. W. Wirth, R. H. Foote and J. R. Coulson. Washington, United States Department of Agriculture. Agriculture Handbook no. 276: 557-625.

Zych, M. (2014). "Pollination biology of Heracleum sphondylium L.(Apiaceae): the

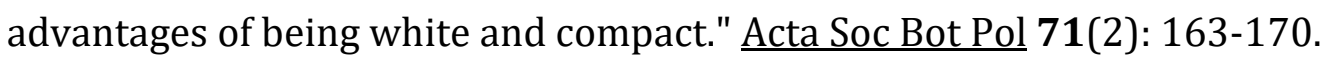


Chapter 2, Table 1: Voucher specimens used to determine exon boundaries for initial probe site selection

\begin{tabular}{|l|l|l|l|l|l|}
\hline & Order & Family & Genus & Epithet & Number of loci \\
\hline Outgroup & Hemiptera & Aphididae & Acyrthosiphon & pisum & 865 \\
\hline Holometabola & Diptera & Culicidae & Aedes & aegypti & 874 \\
\hline Holometabola & Hymenoptera & Apidae & Apis & mellifera & 937 \\
\hline Holometabola & Lepidoptera & Bombycidae & Bombyx & mori & 962 \\
\hline Holometabola & Diptera & Culicidae & Culex & quinquefasciatus & 874 \\
\hline Holometabola & Diptera & Drosophilidae & Drosophila & melanogaster & 855 \\
\hline Holometabola & Hymenoptera & Formicidae & Harpegnathos & saltator & 927 \\
\hline Holometabola & Strepsiptera & Mengenillidae & Mengenilla & moldrzyki & 959 \\
\hline Holometabola & Hymenoptera & Pteromalidae & Nasonia & vitripennis & 916 \\
\hline Outgroup & Anoplura & Pediculidae & Pediculus & humanus & 954 \\
\hline Holometabola & Hymenoptera & Pogonomyrmex & Pogonomyrmex & barbatus & 937 \\
\hline Holometabola & Coleoptera & Cupedidae & Priacma & serrata & 597 \\
\hline Holometabola & Coleoptera & Tenebrionidae & Tribolium & castaneum & 946 \\
\hline
\end{tabular}


Chapter 2, Table 2: Diptera genomes and transcriptomes used to develop probe kit

\begin{tabular}{|c|c|c|c|c|c|c|}
\hline AnalysisName & Genus & Species & Type & Source & Accession e.g. & \\
\hline aedAeg & Aedes & aegypti & Genome & NCBI & AAGE02000001 & http://www.ncbi.nlm.nih.gov/genome/44 \\
\hline anoGam & Anopheles & gambiae & Genome & NCBI & CM000360 & http://www.ncbi.nlm.nih.gov/genome/46 \\
\hline culQui & Culex & quinquefasciatus & Genome & NCBI & AAWU01000001 & http://www.ncbi.nlm.nih.gov/genome/393 \\
\hline droMel & Drosophila & melanogaster & Genome & NCBI & AABU01000001 & http://www.ncbi.nlm.nih.gov/genome/47 \\
\hline lutLon & Lutzomyia & longipalpis & Genome & HGSC & AJWK01000001 & http://www.hgsc.bcm.tmc.edu/project-species-i-Lutzomyia_longipalpis.hgsc \\
\hline mayDes & Mayetiola & destructor & Genome & NCBI & AEGA01000001 & http://www.ncbi.nlm.nih.gov/genome/2619 \\
\hline phlPap & Phlebotomus & papatasi & Genome & WUSTL & AJVK01000001 & http://genome.wustl.edu/genomes/view/phlebotomus papatasi \\
\hline Anabarhynchus & Anabarhynchus & dentiphallus & Transcriptome & 1kite.org & unpublished & http://1kite.org project ID\# INSswpTBHRAAPEI-35 \\
\hline Bibio & Bibio & marci & Transcriptome & 1kite.org & GATJ02 & http://www.ncbi.nlm.nih.gov/Traces/wgs/?val=GATj02 \\
\hline Bombylius & Bombylius & major & Transcriptome & 1kite.org & GATI02 & http://www.ncbi.nlm.nih.gov/Traces/wgs//?val=GATI02 \\
\hline Chrysosoma & Heteropsilopus & ingenuus & Transcriptome & 1kite.org & unpublished & http://1kite.org project ID\# INSswpTAIRAAPEI-19 \\
\hline Episyrphus & Episyrphus & balteatus & Transcriptome & 1kite.org & unpublished & http://1kite.org project ID\# INSnfrTAWRAAPEI-11 \\
\hline Exaireta & Exaireta & spinigera & Transcriptome & 1kite.org & unpublished & http://1kite.org project ID\# INSswpTAERAAPEI-15 \\
\hline Lipara & Lipara & lucens & Transcriptome & 1kite.org & GAZD02 & http://www.ncbi.nlm.nih.gov/Traces/wgs//?val=GAZD02 \\
\hline Meroplius & Meroplius & fasciculatus & Transcriptome & 1kite.org & unpublished & http://1kite.org project ID\# INSytvTAARAAPEI-9 \\
\hline Sicus & Sicus & ferrugineus & Transcriptome & 1kite.org & unpublished & http://1 kite.org project ID\# INShkeTARRAAPEI-46 \\
\hline Triarthria & Triarthria & setipennis & Transcriptome & 1kite.org & GAVA02 & http://www.ncbi.nlm.nih.gov/Traces/wgs//?val=GAVA02 \\
\hline Trichocera & Trichocera & saltator & Transcriptome & 1kite.org & GAXZ02 & http://www.ncbi.nlm.nih.gov/Traces/wgs//?val=GAXZ02 \\
\hline Chrysops & Chrysops & vittatus & Transcriptome & Wiegmann & unpublished & Wiegmann Lab, NCSU; Pers. Comm.. \\
\hline Empis & Empis & snoddyi & Transcriptome & Wiegmann & unpublished & Wiegmann Lab, NCSU; Pers. Comm.. \\
\hline Muscidae & Musca & domestica & Transcriptome & Wiegmann & unpublished & Wiegmann Lab, NCSU; Pers. Comm.. \\
\hline
\end{tabular}


Chapter 2, Table 3: Voucher specimens used in phylogenetic analysis. JSS = Jeff Skevington Specimen. All vouchers deposited in CNC

\begin{tabular}{|c|c|c|c|c|c|c|c|c|}
\hline Family & Subfamily & Tribe & Taxon & $\begin{array}{l}\text { Accession } \\
\text { Number }\end{array}$ & Genbank \# & Biosample \# & Family & Locality \\
\hline Pipunculidae & & & Chalarus spurius & JSS 22746 & KU687412 & SAMN03352425 & Pipunculidae & Spain, Extremadura \\
\hline Pipunculidae & & & Pipunculus sp. ON12 & JSS 24663 & KR260235 & SAMN03352426 & Pipunculidae & Canada, Ontario \\
\hline Platypezidae & & & Platypeza sp. & JSS 24755 & KR260237 & SAMN03352427 & Platypezidae & Canada, Ontario \\
\hline Sepsidae & & & Themira nigricornis & JSS 26210 & KR260243 & SAMN03352428 & Sepsidae & Canada, Ontario \\
\hline Tachinidae & & & Epalpus signifer & JSS 23233 & KR260213 & SAMN03352424 & Tachinidae & Canada, Quebec \\
\hline Syrphidae & Eristalinae & Brachyopini & Sphegina rufiventris & JSS 24645 & KR260242 & SAMN03352330 & Syrphidae & Canada, Ontario \\
\hline Syrphidae & Eristalinae & Callicerini & Callicera montensis & JSS 23232 & KR260209 & SAMN03352268 & Syrphidae & U.S.A., California \\
\hline Syrphidae & Eristalinae & Eristalini & Helophilus fasciatus & JSS 23235 & KR260219 & SAMN03352282 & Syrphidae & Canada, Ontario \\
\hline Syrphidae & Eristalinae & Merodontini & Eumerus sp. & JSS 22745 & KR260216 & SAMN03352286 & Syrphidae & Spain, Extremadura \\
\hline Syrphidae & Eristalinae & Merodontini & Merodon aberrans & JSS 23236 & KR260228 & SAMN03352303 & Syrphidae & Serbia \\
\hline Syrphidae & Eristalinae & Milesiini & Brachypalpus oarus & JSS 17666 & KR260208 & SAMN03352284 & Syrphidae & Canada, Quebec \\
\hline Syrphidae & Eristalinae & Milesiini & Xylota bicolor & JSS 26331 & KR260244 & SAMN03352423 & Syrphidae & U.S.A., Mississippi \\
\hline Syrphidae & Eristalinae & Rhingiini & Cheilosia soror & JSS 22751 & KR260210 & SAMN03352305 & Syrphidae & Serbia \\
\hline Syrphidae & Eristalinae & Rhingiini & Ferdinandea buccata & JSS 26304 & KR260217 & SAMN03352384 & Syrphidae & U.S.A., Tennessee \\
\hline Syrphidae & Eristalinae & Rhingiini & Rhingia nasica & JSS 24659 & KR260238 & SAMN03352342 & Syrphidae & Canada, Ontario \\
\hline Syrphidae & Eristalinae & Volucellini & Copestylum caudatum & JSS 17391 & KR260212 & SAMN03352283 & Syrphidae & U.S.A., New Mexico \\
\hline Syrphidae & Eristalinae & Volucellini & Graptomyza sp. & JSS 25866 & KR260218 & SAMN03352378 & Syrphidae & Malaysia, Sabah \\
\hline Syrphidae & Microdontinae & Microdontini & Microdon tristis & JSS 22763 & KR260229 & SAMN03352280 & Syrphidae & Canada, Ontario \\
\hline Syrphidae & Pipizinae & & Heringia calcarata & JSS 22754 & KR260220 & SAMN03352265 & Syrphidae & Canada, Quebec \\
\hline Syrphidae & Pipizinae & & Pipiza crassipes & JSS 22759 & KR260233 & SAMN03352271 & Syrphidae & U.S.A., Alaska \\
\hline Syrphidae & Pipizinae & & Pipiza nigripilosa & JSS 22762 & KR260234 & SAMN03352277 & Syrphidae & U.S.A., North Carolina \\
\hline Syrphidae & Syrphinae & Bacchini & Baccha elongata & JSS 22758 & KR260206 & SAMN03352270 & Syrphidae & U.S.A., Alaska \\
\hline Syrphidae & Syrphinae & Bacchini & Melanostoma mellinum & JSS 24699 & KR260227 & SAMN03352376 & Syrphidae & Canada, Ontario \\
\hline Syrphidae & Syrphinae & Bacchini & Platycheirus sp. & JSS 24698 & KR260236 & SAMN03352343 & Syrphidae & Canada, Ontario \\
\hline Syrphidae & Syrphinae & Paragini & Paragus haemorrhous & JSS 26268 & KR260231 & SAMN03352381 & Syrphidae & Republic of Korea \\
\hline Syrphidae & Syrphinae & Syrphini & Allograpta obliqua & JSS 26309 & KR260202 & SAMN03352377 & Syrphidae & U.S.A., Mississippi \\
\hline Syrphidae & Syrphinae & Syrphini & Betasyrphus serarius & JSS 25987 & KR260207 & SAMN03352269 & Syrphidae & Malaysia, Sabah \\
\hline Syrphidae & Syrphinae & Syrphini & Citrogramma circumdatus & JSS 25726 & KR260211 & SAMN03352288 & Syrphidae & Indonesia, West Papua \\
\hline Syrphidae & Syrphinae & Syrphini & Epistrophe grossulariae & JSS 18561 & KR260214 & SAMN03352306 & Syrphidae & Canada, Ontario \\
\hline Syrphidae & Syrphinae & Syrphini & Episyrphus balteatus & JSS 26269 & KR260215 & SAMN03352382 & Syrphidae & Republic of Korea \\
\hline Syrphidae & Syrphinae & Syrphini & Leucozona americanum & JSS 23231 & KR260224 & SAMN03352264 & Syrphidae & Canada, Quebec \\
\hline Syrphidae & Syrphinae & Syrphini & Ocyptamus fuscipennis & JSS 26326 & KR260230 & SAMN03352421 & Syrphidae & U.S.A., Mississippi \\
\hline Syrphidae & Syrphinae & Syrphini & Parasyrphus annulatus & JSS 22749 & KR260232 & SAMN03352289 & Syrphidae & Serbia \\
\hline Syrphidae & Syrphinae & Syrphini & Scaeva dignota & JSS 19737 & KR260239 & SAMN03352304 & Syrphidae & Serbia \\
\hline Syrphidae & Syrphinae & Syrphini & Sphaerophoria scripta & JSS 22750 & KR260241 & SAMN03352292 & Syrphidae & Serbia \\
\hline
\end{tabular}




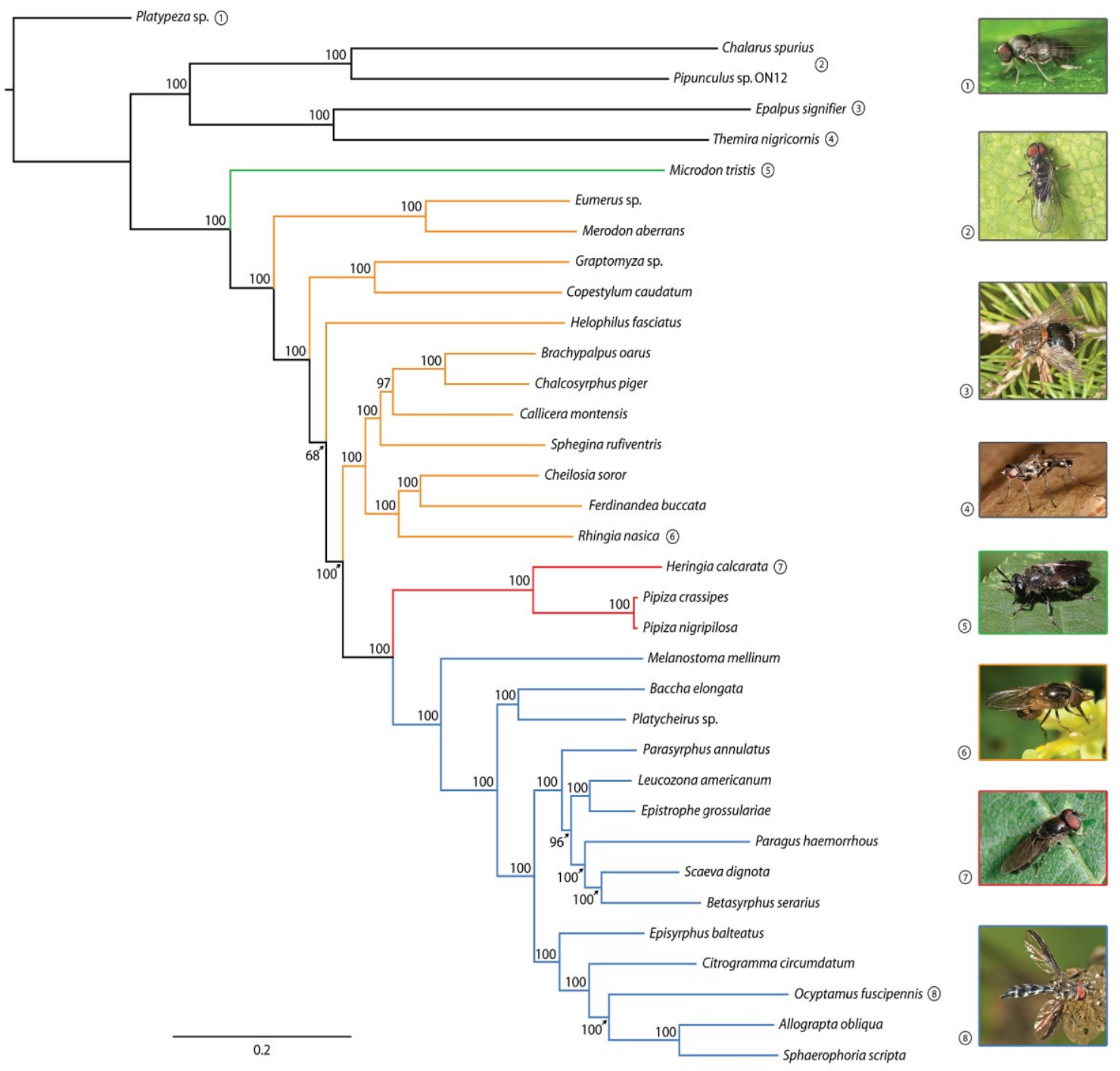

Chapter 2, Figure 1: The ML phylogenetic tree based on the sequenced taxa using RAxML under the model GTR+G. Bootstrap support values are depicted above the nodes. Legend: black: outgroups; green: Microdontinae; orange: Eristalinae; red: Pipizinae; and blue: Syrphinae. Figure by Ximo Mengual. 


\title{
Chapter 3: Revision of Australian Psilota Meigen, 1822 (Diptera: Syrphidae), including descriptions of new species
}

\author{
A. D. Young, J. H. Skevington, W. van Steenis, and F. C. Thompson
}

\section{Author Contributions:}

A. D. Young: Wrote text, wrote final version of key, described all species, took photographs and assembled plates, initially recognized all species authored Young.

J. H. Skevington: Assisted A.D Young with species concepts, manuscript editing and revision, specimen collection.

W. van Steenis: Provided A.D. Young with manuscript species key, initially recognized all species authored Young and van Steenis.

F.C. Thompson: Provided A.D. Young with manuscript species key, initially recognized all species authored Young and Thompson.

\section{ICZN Disclaimer}

In accordance with article 8.2 of the International Code of Zoological Nomenclature, this thesis chapter is not issued for public and permanent scientific record, or for the purposes of zoological nomenclature. Therefore, this document is not published within the meaning of the Code, and the new names given are not valid species names. 


\section{Abstract}

The 30 species of Australian Psilota are revised, with 22 new species described (Psilota aislinni Young sp. nov., Psilota alexanderi Young sp. nov., Psilota apiformis Young and Thompson sp. nov., Psilota auripilosa Young sp. nov., Psilota austropsilota Young and Thompson sp. nov., Psilota azurea Young and Thompson sp. nov., Psilota bicolor Young and Ferguson sp. nov., Psilota brunnipennis Young sp. nov., Psilota calva Young sp. nov., Psilota darwini Young sp. nov., Psilota livida Young and van Steenis sp. nov., Psilota longipilosa Young and Thompson sp. nov., Psilota maculata Young and van Steenis sp. nov., Psilota mcqueeni Young sp. nov., Psilota metallica Young and Thompson sp. nov., Psilota nigripilosa Young sp. nov., Psilota pallidifacies Young and van Steenis sp. nov., Psilota pollinosa Young and van Steenis sp. nov., Psilota solata Young and van Steenis sp. nov., Psilota spathistyla Young sp. nov., Psilota spinifemur Young sp. nov., Psilota zophos Young sp. nov.) and one new record for Australia (Psilota basalis Walker, 1858). Currently recognized but previously described species are redescribed, with the males of Psilota auricauda Curran, 1925 and P. basalis (Walker, 1858) described for the first time. Seven previously described species cannot be recognized; six belong to currently intractable species groups (Psilota erythrogaster Curran, 1926, Psilota hirta Klocker, 1924, Psilota queenslandica Klocker, 1924, Psilota rubra Klocker, 1924, Psilota rubriventris Bigot, 1885, and Psilota shannoni Goot, 1964), and one is known only from a destroyed holotype (Psilota victoria Curran, 1925). 


\section{Introduction}

Psilota Meigen, 1822 is a relatively small genus of little understood eristaline syrphid. The genus is rarely collected and species-poor in the Nearctic (three species), Palaearctic (eight species), and Indomalayan (four species) regions, but is abundantly found in the Australian region, which is the centre of diversity (30 species, 22 of which are newly described herein). Unlike other Psilota, many Australian species are strongly iridescent or otherwise brightly coloured.

What is known of Psilota natural history is based on studies and accounts of few species. In an experiment that compared species abundance in ground-level vs. canopy Malaise traps, adult Psilota atra (Fallen, 1817) were collected significantly more frequently in the canopy traps, suggesting that the species is arboreal (Birtele and Hardersen 2012). Females of likely the same species have also been observed investigating the shady side of tree trunks, and ovipositing in a Cerambyx cerdo L., 1758 (Cerambycidae) emergence hole in a live Quercus L. (Fagaceae) (Speight 2016). Known larvae appear to develop in decaying liquid media. Kassebeer et al. (1998) describe the larvae of what is likely P. atra (Speight 2016), and list the larval habitat as "a pocket of decay containing rotten wood, sap, and water inside a living Pinus L.". Similarly, the New Zealand species Psilota decessum (Hutton, 1901) has been found "inhabiting the gum-fluid retained in the leaf-sheathes" of Phormium tenax J.R. Forst. and G. Forst. (Asphodelaceae) and in decaying matter under the bark of Cordyline australis (G. Forst.) Endl. (Asparagaceae) (Miller 1921), while a member of the Australian species complex Psilota cupreus (Macquart, 1850) have been reared "From the rotten caudex of Xanthorrhoea resinosa Pers." (label data 
from D.K. McAlpine specimen, ANIC). Several species are also associated with termites. Larvae of Psilota tectonae Meijere, 1933 and Psilota bifenestrata Meijere, 1933 were originally collected from the rotting materials in Javan termite nests (Meijere 1933), and Psilota victoria Curran, 1925 females were observed by Hill (1921) ovipositing in an oozing crevice on a termite-infested tree. He also reported that larval development and sometimes pupation occur within the termite galleries, but that mature larvae generally leave the termite galleries and pupate in crevices in the adjacent bark (Hill 1921).

Psilota has received little taxonomic attention since its original description. Smit and Vujić (Smit 2007) provide a review of the Palaearctic species, but no other synthesis of a faunal region or geographic area exists. A table of named world species, including synonyms and reclassified species, is available below (Table 1).

The systematic position of Psilota within Eristalinae is unclear. The only quantitative phylogenetic analysis in which Psilota has been included scored only larval morphological characters, and recovered Psilota within a large clade containing many, but not all, Eristaline genera (Rotheray and Gilbert 1999). This classification has never been widely used. Based on qualitative analysis of adult morphology, Psilota has also been placed within Merodontini (Thompson 1972, Peck 1988), Chrysogasterini (Wirth, Sedman et al. 1965, Shatalkin 1975), or in a monogeneric subfamily (Glumac 1960). Psilota has never been included in a published molecular phylogenetic analysis, but an upcoming six gene analysis places Psilota sister to the remainder of the eristalines excluding the tribes Eumerini and 
Ceriodini (Moran 2018, pers comm.).

The objective of the present study is to describe new Australian Psilota and emend the descriptions of fauna for which males were newly discovered. A partial DNA barcode library is provided, containing all species for which we were able to sequence the 5' end of COI. Photographic plates of external morphology and male genitalia are provided for all species along with a dichotomous key. DNA barcode evidence indicates that both Psilota tristis Klocker, 1925 and Psilota cupreus Macquart, 1850 are species complexes, which will be resolved in a future publication.

\section{Materials and Methods}

\section{Specimen examination}

Adult specimens were generally pinned directly after collecting, or stored in ethanol and later critical-point dried in the case of Malaise trap samples. Clearing and removal of male genitalia were necessary in order to view internal characters useful for classification and species delimitation. The genitalia capsule was removed by cutting between tergites 7 and 8 , and then cleared by heating in $88 \%$ lactic acid overnight. A full materials examined list is provided after each species description. Specimens were borrowed from the following institutions and individuals (collection codes based on Evenhuis 2018): 
Australia

CNC Canadian National Collection of Insects, Arachnics, and Nematodes, Ottawa, Ontario, Canada

CSCA California State Collection of Arthropods, Sacramento, California, USA

DEBU University of Guelph Insect Collection, Guelph, Ontario, Canada

GDCB Greg Daniels Personal Collection

MCZ Harvard University, Museum of Comparative Zoology, Cambridge, Massachusetts, USA

QM Queensland Museum, Brisbane, Queensland, Australia

SAMA South Australian Museum, Adelaide, South Australia, Australia

TMAG Tasmanian Museum and Art Gallery, Hobart, Tasmania, Australia

UQIC University of Queensland Insect Collection, Brisbane, Queensland, Australia

USNM National Museum of Natural History, Washington D.C., USA

VAIC Victorian Agricultural Insect Collection, Victoria, Australia

WADA Western Australia Insect Collection, South Perth, Western Australia, Australia

WVSPC Wouter van Steenis Personal Collection

ZMUC University of Copenhagen Zoological Museum, Copenhagen, Denmark

AM Australian Museum, Sydney, New South Wales, Australia

MVZPC Menno van Zuijen Personal Collection

\section{Specimen photography, measurement, and figures}

Habitus and genitalia photographs for all specimens were taken using a Leica

M205-C stereoscope (Leica Microsystems Inc., Concord, Ontario, Canada) using 0.6x (habitus) and 1.6x (genitalia) lenses. Raw images to be used in depth-of-field photomontages were captured using Leica Application Suite, and final images were created using Zerene stacker (Littlefield 2018). Specimen measurements were taken 
using the Leica measurement module in Leica Application Suite. Figures and maps are presented alphabetically. Maps include points from all specimens examined, and were produced using Simplemappr (Shorthouse 2010).

\section{DNA Sequencing}

The right hind leg was removed from all recently collected (post 2000) specimens and sent to the University of Guelph Biodiversity Institute of Ontario for sequencing of the $5^{\prime}$ end of the Cyctochrome $c$ Oxidase I mitochondrial gene (COI), or Barcoding region, following protocols published in (Hajibabaei, deWaard et al. 2005). DNA extraction and sequencing from older specimens was performed by Scott Kelso at the Canadian National Collection of Insects (CNC) using a modified version of the same protocol, with custom primers:

$\begin{array}{lll}\text { Primer Name } & \text { Primer Design } & \text { Primer Sequence } \\ \text { Heb-F } & \text { Folmer et al. 1994 } & \text { GGT CAA CAA ATC ATA AAG ATA TTG G } \\ \text { COI-Fx-A-R } & \text { Kelso (in prep) } & \text { CGD GGR AAD GCY ATR TCD GG } \\ \text { COI-Fx-B-F } & \text { Kelso (in prep) } & \text { GGD KCH CCN GAY ATR GC } \\ \text { COI-Fx-B-R } & \text { Kelso (in prep) } & \text { GWA ATR AAR TTW ACD GCH CC } \\ \text { COI-Fx-C-F } & \text { Kelso (in prep) } & \text { GGD ATW TCH TCH ATY YTA GG } \\ \text { COI-780R } & \text { Gibson et al. 2011 } & \text { CCA AAA AAT CAR AAT ARR TGY TG }\end{array}$

These custom primers sequence the Barcoding region in three segments, allowing more badly degraded DNA to be successfully sequenced. All sequence data are 
stored on the BOLD website (www.boldsystems.org) in the Psilota of Australia (AUSPSIL) dataset, available at http://www.boldsystems.org/index.php/Public SearchTerms?query=DS-AUSPSIL. With material sequenced at CNC, raw sequence reads were scored using Sequencer 5.4.6 (2018), and aligned using Mesquite (Maddison and Maddison 2010). In some cases BOLD alignments were also manually checked and corrected using Mesquite.

\section{Definitions}

Syrphidae-specific terminology follows Thompson (1999), while all other morphological terminology follows Cumming and Wood (2017). A visual glossary of common terminology used is available in Figures 2-4.

\section{Species concept}

This revision was performed under the assumption that the phylogenetic species concept is an appropriate measure of speciation for sexually reproducing insects. Although phylogenetic analysis of Psilota has not yet been performed, COI Barcode data indicates that species described herein represent phylogenetically distinct units that are not interbreeding.

\section{Results and Discussion}

\section{Barcoding and species complexes in Psilota}

A neighbour-joining tree of all available Australian Psilota DNA barcodes is

presented in Figure 43. From the tree it is evident that a substantial "barcoding gap" 
(Čandek and Kuntner 2015) of >2\% difference exists between most species, allowing them to be identified readily using COI alone if necessary. The only two successfully barcoded species that may prove to be unreliable to separate based on COI alone are Psilota coerulea Macquart, 1846 and Psilota aislinni Young, sp. nov..

The neighbour-joining tree also shows that at least two species, Psilota tristis Klocker, 1924 (a black-bodied species with a simple hind femur) and Psilota cupreus Macquart 1850 (a species with a red abdomen), are species complexes. What is here referred to as Psilota tristis appears to be comprised of at least 19 separate barcode BINs, while Psilota cupreus is comprised of at least six. Various names exist for each of these two morphotypes (see descriptions for P. tristis and P. cupreus as well as species not recognized for more information), but existing holotypes are universally older specimens in poor condition, and cannot be morphologically associated with a specific species group circumscribed by COI data at the present time. Therefore, we have chosen not to attempt to redescribe or synonymize each of the various species that fall within these two complexes. Instead, we refer to all entirely black-bodied Psilota with an unmodified hind leg as Psilota tristis, the oldest available name matching that morphotype, and likewise treat all Psilota with a red abdomen as Psilota cupreus. All other names that may apply to the two species groups are addressed under the species not recognized subheading below. These two species complexes and all associated names will be addressed in a future paper, using a combination of further DNA barcoding and morphology. 


\section{Taxonomic treatment of Psilota}

Psilota Meigen, 1822

Psilota Meigen, 1822: 256. Type species, Psilota anthracina Meigen, 1822 (monotypy).

=Coiloprosopa Macquart, 1850: 449. Type species, Coiloprosopa nitida Macquart, 1850 (monotypy).

=Emmyia Klocker, 1924: 55. Type species, Emmyia queenslandica Klocker, 1924 (original designation).

=Parapsilota Hardy, 1933: 16 (Psilota subgenus). Type species, Psilota viridis Macquart, 1847 (original designation).

Diagnosis: Eye pilose, face pilose except in Psilota austropsilota sp. nov., straight to slightly concave with epistoma projecting anteriorly. Spurious vein rudimentary to completely absent. Meron with a tuft of fine pile anteroventral to posterior spiracle, otherwise bare. Male surstylus with narrow, curved outer lobe and broader inner lobe.

Description: Head: Rounded in frontal view. Eye pilose, broadly holoptic in male and dichoptic in female. Face pilose laterally (except in Psilota austropsilota sp. nov.), lacking tubercle, straight to slightly concave with epistoma projecting anteriorly, pollinose or completely bare laterally, shining and free of pollinosity centrally. Frontal prominence not produced. Gena not well developed. Frons short, 
broad, pilose, slightly longer than vertical triangle. Vertex pilose, ocellar triangle approximately equilateral. Antenna inserted at $1 / 2$ the height of the head, with scape and pedicel short; postpedicel ovoid, or subtriangular and tapering to a point, with sensory pit on medial surface; arista bare.

Thorax: Scutum slightly longer than wide; scutellum about twice as wide as long, with apical marginal rim, subscutellar fringe present; pleuron entirely pollinose to entirely bare and shining; postpronotum, anepisternum, anepimeron, and katepisternum pilose, katepimeron, katatergum, and metaepisternum bare, anatergum with fine pile, meron with a tuft of fine pile anteroventral to posterior spiracle, metasternum pilose. Legs sometimes with hind femur somewhat thickened and/or with an apicoventral carina armed with thickened setula, hind tibia sometimes with a small basoventral spur, legs otherwise unmodified. Wing entirely microtrichose to extensively bare, usually hyaline but sometimes with an anteromedial or apical darkened spot; spurious vein rudimentary or entirely absent and represented only by a crease; cell $\mathrm{R}_{1}$ open to wing margin, vein $\mathrm{R}_{4+5}$ straight; alula well developed, plumula present and single branched.

Abdomen: oval, approximately 0.75 times as broad as long.

Male genitalia: Usually symmetric, although some species with slight asymmetry of the surstyli; cercus rectangular to sub-triangular; surstylus forked, usually with a 
broad, paddle-shaped inner medial lobe and a thin, downward curved lateral lobe; aedeagal apodeme crescent shaped, sometimes with a ventral hook; gonopod rounded, with dense patch of thickened setae on the dorso-medial surface.

\section{Key to Australian species of Psilota}

1. Face bare, abdominal tergite 3 with a distinct tubercle. Purplish species (Fig. 11) ... Psilota austropsilota, sp. nov.

- $\quad$ Face pilose, abdominal tergite 3 never with tubercle. Colouration variable. ... 2

2. Hind femur with an apicoventral carina, either low and triangular, distinct and triangular, or distinct and lobe-like ... 13

Hind femur simple, with at most an apicoventral patch of setulae ... 3

3. Face yellow. Abdomen broadly oval, tergites 3 and 4 almost entirely yellow, with long, dense, pale yellow pile (Fig. 7) ... Psilota apiformis sp. nov. Face black or iridescent blue/green, never yellow. Abdomen not as broad. If tergites 3 and 4 mostly yellow, never with long, dense, pale yellow pile. If tergites with long, dense, pale yellow pile, then tergites 3 and 4 entirely black in ground colour. ... 4 
4. Entire body strongly iridescent. Head and thorax blue and green iridescent, abdomen red and green iridescent. Antenna entirely orange (Fig. 25). ... Psilota metallica sp. nov.

- $\quad$ Entire body usually not iridescent. If iridescent, than thorax and abdomen always blue, never green and red. Antenna variably coloured. ... 5

5. Wing with anterior brown vitta. Tergite 2 with two yellow/brown triangular maculae, ranging from less than half the length of tergite 2 to spanning almost the entire length of the tergite (Fig. 13). ... Psilota basalis (Walker)

- $\quad$ Wing never with anterior brown vitta. Tergite 2 with or without yellow markings, but never with two triangular maculae. ... 6

6. Tergite 2 never with yellow markings. ... 8

- $\quad$ Tergite 2 either almost entirely yellow (Fig. 14), or yellow with a large medial black macula (Fig. 23).... 7

7. Tergite 2 almost entirely yellow, usually with a posterior black margin. Tergites 1, 3 and 4 black (Fig. 14).... Psilota bicolor sp. nov. 
- $\quad$ Tergites 1-4 yellow, Tergites 3 and 4 each with a large, medial, triangular black macula (Fig. 23). ... Psilota maculata sp. nov.

8. Thorax black. Abdomen black or red, at most faintly iridescent blue on posterior margins. Postpedicel usually dark brown or black, occasionally dull orange with a brown dorsal edge. ... 10

- $\quad$ Thorax and abdomen iridescent blue. Postpedicel entirely bright orange. ... 9

9. Abdomen faintly iridescent blue, almost black. Calypter entirely pale (Fig. 21). ...Psilota livida sp. nov.

Abdomen iridescent indigo blue, extremely bright. Calypter dark at least at margin, usually entirely dark brown (Fig. 12). ... Psilota azurea sp. nov.

10. Abdominal tergites 2 to 4 mostly or entirely red (Fig. 18).... Psilota cupreus complex

- $\quad$ Abdominal tergites entirely black, sometimes with faint iridescent blue posterior margins or with long bright yellow-orange pile. ... 11 
11. Face faintly iridescent blue. Pollinosity on face restricted to two lateral triangles below antenna. Tergites 2 to 4 with faint posterior iridescent blue margins (Fig. 16). ... Psilota calva sp. nov.

Face black. Pollinosity on face uniform. Tergites 2 to 4 never with faint iridescent blue posterior margin. ... 12

12. Scutellum entirely to almost entirely white pilose, with at most two apical black pili (Figs. 8,9). Tergites 3 to 5 with long, dense, bright orange/yellow pile in female only (Fig. 9).... Psilota auricauda Curran

Scutellum with mixed black and white pile, or entirely black pilose. Female never with long, dense, orange/yellow pile on tergites 3 to 5 (Fig. 33).... Psilota tristis complex

13. Face faintly iridescent blue. Pollinosity on face restricted to two lateral triangles below antenna. Hind femur with a distinct apicoventral equilateral carina (Fig. 24). ... Psilota mcqueeni sp. nov.

- $\quad$ Face colour variable. Pollinosity on face uniform. Hind femur with either a low triangular carina or a distinct lobe-like carina. ... 14

14. Hind femur with distinct lobe-like carina. ... 19 
- $\quad$ Hind femur with low triangular carina. ... 15

15. Body metallic blue. Face with extremely long, dense, yellow pile (Fig. 22)....

\section{Psilota longipilosa sp. nov.}

- $\quad$ Body black. Face with pilosity variable, but never with long, dense, yellow pile. ... 16

16. Wing with apical tip dark brown. Large black species (Fig. 15).... Psilota brunnipennis sp. nov.

- Wing colourless or with anterior half tinted brown. Never with distinct apical brown spot. Size variable, legs often orange. ... 17

17. Facial pile entirely pale (Fig. 10).... Psilota auripilosa sp. nov

- $\quad$ Facial pile at least partly black, usually with pile closest to eye pale (Fig. 26). $\ldots 18$

18. Male genitalia with inner lobe of surstylus broadest near base, very nearly parallel-sided (Fig. 42A).... Psilota spathistyla sp. nov.

- $\quad$ Male genitalia with inner lobe of surstylus paddle-shaped, broadest near apex (Fig. 40G). ... Psilota nigripilosa sp. nov. 
19. Tergites 3 and 4 with long, dense, tangled, bright yellow pile. Black species with orange legs (Fig. 30). ... Psilota solata sp. nov.

Tergites 3 and 4 with black and/or white pile, never with long, dense, tangled, bright yellow pile. Body colour variable. ... 20

20. Face dull orange to brown. Thorax metallic blue. Abdomen metallic green (Fig. 28). ... Psilota pallidifacies sp. nov.

- $\quad$ Face black. Thorax and abdomen variable. ... 21

21. Carina on hind femur entirely covered in thick black setulae on medial side. Frons pale pilose. Abdomen faintly iridescent green (Fig. 32). ... Psilota spinifemur sp. nov.

- $\quad$ Carina on hind femur usually with setulae only on ventral rim. If medial side of carina completely covered in setulae, than frons black pilose. Abdomen variable. ... 22

22. Body iridescent blue/green in ground colour. Pleuron usually shining, pollinosity free. Frons pale or black pilose. If pleuron pollinose, than frons always pale pilose $\ldots 24$ 
- $\quad$ Body black in ground colour. Pleuron densely pale pollinose. Frons black pilose. ... 23

23. Halter orange (Fig. 27).... Psilota nitida (Macquart)

Halter dark brown. ... 29

24. Pleuron white pollinose and white pilose (Fig. 29). ... Psilota pollinosa sp. nov.

- $\quad$ Pleuron shining and free of pollinosity, white or black pilose. ... 25

25. Calypter pale, at most with dark brown rim. If calypter with brown rim, then pleural pile white. ... 28

- $\quad$ Calypter entirely dark brown. Pile fringe on rim of calypter pale or dark. Pleural pile black or white. ... 26

26. Pleural pile entirely black. Pile on rim of calypter entirely dark brown (Fig. 17). ... Psilota coerulea Macquart

- $\quad$ Pleural pile entirely white or with some black pili mixed in with white. Pile on rim of calypter not entirely dark brown. ... 27 
27. Pile on rim of calypter yellow, except at corner where upper and lower calypter meet, where pile is dark brown. Pleural pile mostly white with some interspersed black pili (Fig. 5). ... Psilota aislinni sp. nov.

Pile on upper calypter entirely white, pile on lower calypter entirely dark brown. Pleural pile white (Fig. 6). ... Psilota alexanderi sp. nov.

28. Legs partly orange, fore and mid tibia almost entirely orange. Calypter entirely white. Frons entirely pale pilose (Fig. 19). Male genitalia with outer lobe of surstylus smoothly curved downwards over entire length (Fig. 39A).

\section{... Psilota darwini sp. nov.}

Legs dark, with only extreme apex of femora and base of tibiae orange. Calypter often with a dark brown rim. Frons entirely pale pilose or with some black pili interspersed (Fig. 34). Male genitalia with outer lobe of surstylus bent downwards sharply at $1 / 3^{\text {rd }}$ its total length, with apex bent backwards towards base of surstylus (Fig. 42E).... Psilota viridis Macquart

29. Male genitalia with apex of outer lobe hooked sharply downwards towards base of surstylus (Fig. 39C). ... Psilota femoralis Schiner Male genitalia with outer lobe curved smoothly downwards (Fig. 42G).... Psilota zophos n. sp. 


\section{Species Descriptions}

\section{Psilota aislinni Young sp. nov.}

(Species plate: Fig. 5, Genitalia: Fig. 36A+B, Map: 1)

Body length: $11.6-12.7 \mathrm{~mm}$.

Diagnosis: Metallic blue species with frons pale pilose, pleuron shiny and white pilose, with a few black pili on the lower katepisternum. Hind femur with a distinct rounded apicoventral setulose carina. Hind tibia simple. Calypter brown, pile on border of calypter yellow except at corner where upper and lower caypter meet, where pile is dark brown. Tergite 4 white pilose.

Description: very similar to Psilota coerulea, differing as follows:

Thorax: Pile on pleuron mostly white with a few black pili on the lower katepisternum, posterior anepisternum, and anepimeron. Scutum and scutellum entirely black pilose. Wing colourless, almost entire microtrichose with only a bare area on the apical half of cell bm. Shaft of halter yellow with knob dark brown. 
Calypter brown, pile on border of calypter yellow except at corner where upper and lower caypter meet, where pile is dark brown.

Genitalia: Epandrium compact, about as long as tall. Cercus approximately triangular. Outer lobe of surstylus inserted below mid-height of inner lobe, curved downwards sharply at $1 / 4^{\text {th }}$ its length, slightly swollen at apex. Inner lobe of surstylus broad, approximately 4 times as long as wide, with slight ventral excavation at midpoint, otherwise gradually broadening towards a rounded apex. Phallapodeme with a small ventral projection. Gonostylus broad, no darker than remainder of genitalia.

Discussion: Nothing is known about the biology of this species. Although it closely resembles Psilota coerulea, the calypter colour character appears to be invariant and is supported by DNA Barcodes.

Etymology: This species is named after my partner, Aislinn Wyatt. Throughout all my years of syrphidology and post-secondary education, Ais has the most loving partner anyone could ever ask for, as well as the occasional field assistant. 
Specimens Examined: HOLOTYPE Material: AUSTRALIA: Queensland: Mt. Marlay, near Stanthorpe, S.E.Q., -28.655786 151.947128; 6-9.XI.1984, coll.D.K. Yeates; UQIC_Reg94644; (1ðึ QM). Paratypes: AUSTRALIA: Queensland: same data as holotype, UQIC_Reg94645 (1ðึ, QM); The Blunder, -27.526975 153.040322, 29.IX.1968, C.F. Ashby, USNM_ENT249823, (10ิ, ANIC).

\section{Psilota alexanderi Young sp. nov.}

(Figs. 1-4, Species plate: Fig. 6, Genitalia: Fig. 36C+D, Map: 2)

Body length: $10.2-13.5 \mathrm{~mm}$.

Diagnosis: Metallic blue species with frons pale pilose, pleuron shining and partly white pilose. Hind femur with a distinct rounded apicoventral setulose carina. Hind tibia with a basoventral spur. Calypter entirely dark brown, with pile on the border of the upper calypter white. Tergite 4 black pilose.

Description: very similar to Psilota coerulea, differing as follows:

Thorax: Legs entirely dark, somewhat metallic blue. Hind tibia with a small basoventral spur. Pile on pleuron almost entirely white, sometimes with individual black pili on the posterior anepisternum, anepimeron, and/or the ventral half of the 
katepisternum. Halter dark brown. Calypter entirely dark brown, with pile on the border of the upper calypter contrasting white.

Abdomen: Abdomen dark metallic blue. Tergites with pile very short. Pile on tergite 3 almost entirely black, with only anterior rim of tergite white pilose. Tergite 4 entirely black pilose.

Genitalia: Epandrium compact, about as long as tall. Cercus approximately triangular. Outer lobe of surstylus inserted just above mid-height of inner lobe, curve downwards sharply at $1 / 4^{\text {th }}$ its length, curving smoothly back towards base of surstylus, thinning at apex. Inner lobe of surstylus subrectangular, approximately 4 times as long as high, rounded at apex. Phallapodeme with small, broad ventral projection. Gonostylus broad, no darker than remainder of genitalia.

Discussion: This western species has been collected hilltopping on Mount Barker. The hilltop is dominated by dry sclerophyll forest and has a communications tower on it. The habitat around the hilltop is largely agricultural. Psilota alexanderi adults were all on an elevated rise just west of the communications array. This summit of the rise is rocky and open with Eucalyptus trees around the clearing. The flies were hovering about 3-4m above the rocks along the edge of this clearing. 
Etymology: This species is named for Alexander Skevington, who co-collected part of the type series. Though young in years, Alexander is already an avid field biologist and nature photographer.

Specimens Examined: HOLOTYPE Material: AUSTRALIA: Western Australia:

Summit of Mount Barker, -34.655691 117.647081, hilltop, 26.I.2015, J.H., A.M. and

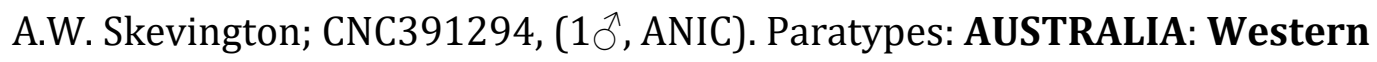

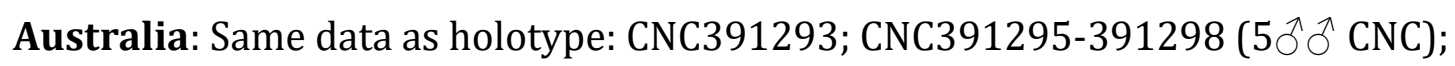
Capel District, 18 miles South of Bunbury, -33.510783 115.641533, 1957, A. Snell,

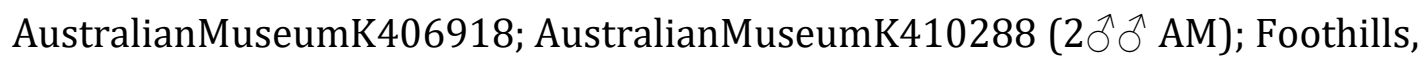
Kelmscott, -32.119000 116.026000, 21.X.1958, J. Baldwin, SAMADatabaseNo.29-

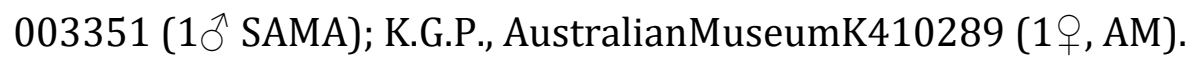

Psilota apiformis Young and Thompson sp. nov. (Species plate: Fig. 7, Genitalia: Fig. 36E+F, Map: 3)

Body length: 9.7-12.2 mm.

Diagnosis: Face yellow. Pleuron obscurely dull orange to brown, scutum black with obscure dull orange to brown lateral stripes. Hind leg simple. Abdomen 


\section{broadly oval, tergites 3 and 4 almost entirely yellow, with long, dense, pale yellow pile.}

Description: Head: Frons and face yellow, very thinly pollinose at anterior margin of eye, otherwise bare. Face and frons with yellow pile, vertex with a tuft of long yellow/orange pile. Antenna orange. Eye densely pilose, pile entirely yellow.

Thorax: Legs entirely dull orange to brown. Hind femur unmodified, with only a few weak, black setulae apicoventrally. Pleuron obscurely dull orange to brown, entirely shining. Pile on pleuron orange dorsally, most katepisternal pile black. Scutum dull orange to brown laterally, with large dark brown/black area medially. Scutal pile mostly yellow, with some black pile anterior to scutellum. Scutellum dark brown, with pile mostly black, but with some yellow pile anteriorly. Wing colourless, with bare areas on the anterior half of cell c and cua, mostly bare on cells bm and r. Halter orange.

\footnotetext{
Abdomen: Broadly oval. Tergite 1 entirely dark brown, tergite 2 yellow laterally and ventrally, dark brown anteriorly and centrally, tergite 3 almost entirely yellow with faint dark brown markings laterally, tergite 4 dark brown with a central yellow stripe. Tergites 3 and 4 entirely covered in pale yellow pollinosity, obscuring ground colour beneath. Tergites 2-4 covered with short, dense, pile. Pile on tergite 2 dark
} 
anteriorly and medially in an area corresponding with the central dark brown marking, all other pile of tergites pale yellow. All sternites yellow.

Genitalia: Epandrium compact, about as long as tall. Cercus approximately triangular. Outer lobe of surstylus inserted near top of outer lobe, abruptly curved downwards at $2 / 3^{\text {rds }}$ its length, swollen at apex. Inner lobe of surstylus broad, approximately 6 times as long as wide, with slight swelling at base, otherwise smoothly broadening towards a rounded apex. Aedeagal apodeme smooth. Gonostylus constricted medially and abruptly darkened.

Discussion: Nothing is known about the biology of this species.

Etymology: Api- derives from Apis, the scientific name for bees, and formis derives from the Latin "forma", meaning "shape, figure, form, or appearance". This is in reference to the bee-like appearance of Psilota apiformis, which may grant it some measure of protection from predators.

\section{Specimens Examined: HOLOTYPE Material: AUSTRALIA. New South Wales:}

Goondera Ridge, Royal National Park, -34.114342 151.046586, 22.viii.1976, G. and A. Daniels, GDCBReg_19538 (1へ^, GDCB). Paratypes: AUSTRALIA: New South Wales: Same data as holotype: GDCBReg_19539-19542 (4へðえ, GDCB); Goondera 
Ridge, Royal National Park, -34.114342 151.046586, 2.x.1978, G. Daniels,

GDCBReg_19543 (1ðð, GDCB); Clyde Mts. nr. Braidwood, -35.545950 149.950408, 26.x.1960, S.J. Paramonov, USNM_ENT_00249838 (10^, USNM); Stanwell Tops, -

34.219903 150.991808, 1.x.1978, G. Daniels, GDCBReg_19544-19545 (2ðð

Queensland: Mount Marlay nr, Stanthorpe , -28.653583 151.946908, 22.ix.1985,

D.K. Yeates, UQIC_Reg94622 (1ð, QM); Gordon, x.1935, ANIC29034936 (1ð, ANIC).

Psilota auricauda Curran, 1926

(Species plate: Figs. 8+9, Genitalia: Fig. 36G+H, Map: 4)

Body length: $12.0-15.4 \mathrm{~mm}$.

Diagnosis: Sexually dimorphic. Both sexes with cuticle entirely shining black, scutum and pleuron with faint blue sheen. Scutellum densely white pilose, with at most a few scattered dark pili on the apical rim. Halter orange. Calypter pale, with brown rim, calypter pile pale. Hind leg simple. Female only with bright yellow pile on tergites 3, 4 and 5 . Yellow pile on apical half of tergite 4 long and dense.

Description: Head: Head black, white pollinose. Face white pilose, frons and vertex black pilose. Antenna brown, postpedicel oval, approximately 1.5 times as long as 
wide. Eye densely pilose, pile white on lower half and dull orange to brown on upper half.

Thorax: Thorax black, faintly reflective blue. Femora dark brown, tibiae and tarsi dull orange to brown. Hind femur unmodified, with only a few weak, black setulae apicoventrally. Pleuron white pollinose. Pile on pleuron and scutellum entirely pale, with at most a few black pili on apical rim of scutellum. Pile on scutum mixed black and pale yellow. Wing mostly colourless, with only stigma and cell sc brown. Cells bc, c, and bm entirely bare. Anterior half of cells br, cua, $\mathrm{m}_{4}$, and $\mathrm{dm}$ bare. Alula entirely microtrichose. Halter orange. Calypter pale with a dark brown border.

Abdomen: Male: Abdomen black. Tergite 2 mostly pale pilose. Tergite 3 with some dark pile, mostly pale pile laterally. Tergite 4 mostly dark pilose.

Female: Abdomen black. Tergite 2 white pilose. Tergites 3, 4 and 5 yellow pilose. Pile of tergite 3 and anterior half of tergite 4 sparse. Pile on posterior half of tergite 4 and all of tergite 5 long and dense.

Genitalia: Epandrium compact, about as long as tall. Cercus approximately triangular. Outer lobe of surstylus inserted near the top of the inner lobe, thin, and abruptly curved downwards at about $1 / 3^{\text {rd }}$ its length. Inner lobe of surstylus long, 
thin, and straight, approximately 5 times as long as wide (at base), slightly swollen at base, otherwise parallel sided over entire length, somewhat blunt tipped. Aedeagal apodeme with a small, downward facing spur on ventral side. Gonopods slightly darkened.

Discussion: This is the first time the male of this species has been described, as the original description by Curran was from female specimens only.

Specimens Examined: AUSTRALIA. Australian Capital Territory: Canberra, 35.282000 149.128686, 23.xi.1959, K.R. Norris, ANIC29034797 (1ㅇ, ANIC); New South Wales: Cheltenham, -33.758389 151.075689, xi-xii.1949, N.W. Rodd, AustralianMuseumK407023 (1ㅇ, AM); Dorrigo, -30.365542 152.738950, W. Heron, Jeff_Skevington_Specimen45813 (1ㅇ, USNM); Gibraltar Ra. N.P., Waratah track, 29.5 152.333333, on Leptospermum sp. Blossom, 10-12.xi.1984, D.K. Yeates, UQIC_Reg94618 (19, UQIC); Queensland: 8 km West of Tyalgum, -28.369994 153.208767, 24.ix.1983, N.W. Rodd, AustralianMuseumK407296 (1우, AM); Amiens State Forest near. Stanthorpe, -28.600522 151.799608, 24-26.xi.1981, UQIC_Reg94619 (1ㅇ, UQIC); Beerburrum, -26.970639 152.968256, 16.ix.1971, E.C. Dahms, QM_REG._NO._T223163 (1ㅇ, QM); Brisbane, -27.526975 153.040322, 15.ix.1915, D.H. Colless, ANIC29014845 (1우, ANIC); 23.x.1911, H. Hacker, ANIC29034792 (19, ANIC); 24.ix.1914, H. Hacker, QM_REG._NO._T223161 (1우, QM); 25.ix.1911, H. Hacker, QM_REG._NO._T223159 (19, QM); 25.ix.1916, H. Hacker, 
QM_REG._NO._T223160 (1ㅇ, QM); 25.ix.1919, H. Hacker, CNC_Diptera255064 (1옹 ANIC); 7.ix.1927, J. Mann, UQIC_Reg94822 (1ㅇ, UQIC); 7.ix.1941, C.F. Ashby, ANIC29034790 (1우, ANIC); ix.1929, G.H. Hardy, AustralianMuseumK407241,

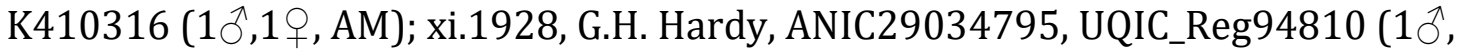
UQIC , 1ㅇ, ANIC); Camira near Ipswich, -27.634853 152.915925, 12.ix.1981, G. Daniels, GDCBReg_19612 (1우, GDCB); Canungra, -28.017142 153.164708, 30.iii.1937, R.F. Langdon, UQIC_Reg94620 (1우, UQIC); Cimara, nr. Ipswich, 27.493986 152.944925, 12.ix.1981, G. Daniels, GDCBReg_19613 (1q, UQIC); Clarence, Blue Mountains, -33.482308 150.224264, 21.xi.1977, N.W. Rodd, AustralianMuseumK407297 (1ㅇ, AM); Cleveland, -27.533333 153.266667, viii.1924, G.H. Hardy, ANIC29034796 (19, ANIC); Dayboro, 0.5 km South, 27.200000 152.816667, 18-19.ix.1999, S.G. Evans, QM_REG._NO._T223162 (1\%, QM); Lansdowne, -31.758753 152.534258, Dry sclerophyll, 19.i.1981, G. Williams, AustralianMuseumK407022 (1ㅇ, AM); Mt. Marlay, near Stanthorpe, S.E.Q., 28.655786 151.947126, 6.xi.1984, D.K. Yeates, UQIC_Reg94621 (1ð, UQIC); Mt. Tinbeerwah near Cooroy, -26.384206 152.966639, 6.ix.1980, G. Daniels, GDCBReg_19610-19611 (2웅, GDCB); One Tree Hill, Brisbane, -27.526975 153.040322, 12.xii.1925, A. Musgrave, AustralianMuseumK407024 (1 $\uparrow$, AM); Rockpool Gorge Mt. Walsh Nat. Park, Biggenden, -25.605889 152.027519, 4.x.1976, H. Frauca, CNC_Diptera253687 (1ð̂, ANIC); Rockpool Gorge, Mount Walsh National Park, Biggenden, -25.605889 152.027519, 4.x.1976, H. Frauca, ANIC29034794, CNC_Diptera255065-255066, USNM_ENT249821-249822 (1 $\overbrace{}^{\lambda}, 4$ 우 $ᄋ$, ANIC); S.Q., on Leptospermum, 21.ix.1959, Mackerras, UQIC_Reg94564 (1ðð, UQIC); Stanthorpe, - 
$28.657928151 .930344,1924$, Date is ambiguous reads "1.10.24", ANIC29034791

(1우, ANIC); Stockyard Creek, SE of Capalaba, -27.550000 153.166667, [Latitude] $27^{\circ} 33^{\prime}$ S [Longitude] $153^{\circ} 10^{\prime}$ E 22.ix.1985, G. and A. Daniels, GDCBReg_19561 (1ð’, UQIC); Sunnybank, -27.578997 153.059006, 27.viii.1950, I.M. Mackerras, ANIC29034793 (1ㅇ, ANIC); The Blunder, Brisbane, -27.526975 153.040322, 18.ix.1966, C.F. Ashby, CNC_Diptera255062 (19, ANIC); 2.x.1966, C.F. Ashby, CNC_Diptera255061 (1へّ̂, ANIC); 8.ix.1962, C.F. Ashby, CNC_Diptera255063 (1우, ANIC); 26.ix.1966, C.F. Ashby, ANIC29034788 (1, ANIC); 4.x.1968, C.F. Ashby, ANIC29034787 (1ㅇ, ANIC); Bluff Range S. [South] Slope, Biggenden, -25.600000 152.050000, 1000m, 15.viii.1973, H. Frauca, ANIC29034789 (1ㅇ, ANIC).

Psilota auripilosa Young and van Steenis sp. nov. (Species plate: Fig. 10, Genitalia: Fig. 37A+B, Map: 5)

Body length: $11.6-15.5 \mathrm{~mm}$.

Diagnosis: Face black, densely white pollinose, with white pile. Frons and vertex black pilose. Thorax black. Hind femur with a low triangular apicoventral setulose carina. Hind tibia with basoventral spur. Calypter with dark brown border. Halter pale. Abdomen black. Lateral edges of tergite 3 with many pale pili. Male genitalia with inner lobe narrow, nearly parallelsided, broadening slightly and regularly over entire length. The white facial 
pile of $P$. auripilosa distinguishes it from similar species, Psilota spathistyla and Psilota nigripilosa.

Description: very similar to $P$. spathistyla, differing as follows:

Head: Head black, face pale pollinose. Face pale pilose.

Abdomen: Abdomen black. Tergite 2 mostly yellow pilose, with some central black pili at posterior border. Tergite 3 yellow pilose laterally, black pilose medially. Tergite 4 with mixed black and yellow pile.

Genitalia: Epandrium compact, slightly broader than long. Cercus approximately triangular. Outer lobe of surstylus inserted near top of inner lobe, curved smoothly downwards at midlength, and then swollen at apex. Inner lobe of surstylus narrow, nearly parallel-sided, broadening slightly and regularly over its entire length, with the apex rounded. Phallapodeme with large ventral projection. Gonostylus thick, not darkened.

Discussion: This species has been collected on Cuttsia viburnea F. Muell. (Rousseaceae) and on Leptospermum minutifolium C.T. White (Myrtaceae) blossoms. 
Etymology: auri- derives from the Latin aurum, which means "gold" or "lustre", and pilosa derives from the scientific term pile, which refers to the hair-like structures covering some tergites in Syrphidae. This is in reference to the bright, pale pile on the face of Psilota auripilosa that distinguishes it from similar species, Psilota spathistyla and Psilota nigripilosa.

\section{Specimens Examined: HOLOTYPE Material: AUSTRALIA: New South Wales; 3}

km Northeast, Bilpin, Blue Mountains; -33.484094 150.552878; N.W. Rodd;

3.XII.1986; AustralianMuseumK410125 (1ðへ, AM). Paratypes: AUSTRALIA. New

South Wales: 3 km North of Lansdowne, -31.758753 152.534258, on Cuttsia viburnea, 16.xii.1991, G. Williams, AustralianMuseumK408020 (1ठ, AM); 4 m. N. of Bateman's Bay, -35.658458 150.177744, 14.x.1952, S.J. Paramonov,

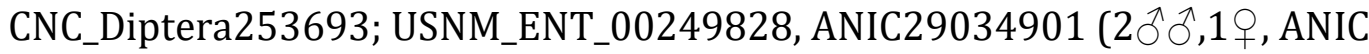
USNM); 20.x.1953, S.J. Paramonov, ANIC29034890 (1へ̂, ANIC); 29.ix.1959, S.J. Paramonov, ANIC29034900 (1§, ANIC); 22.x.1952, S.J. Paramonov, CNC_Diptera253694 (19, ANIC); 20.x.1952, S.J. Paramonov, CNC_Diptera255080

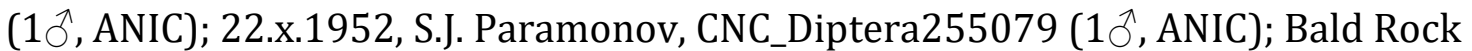
N.P., -28.860783 152.055686, on Leptospermum minutifolium blossom, 6-7.xi.1984, D.K. Yeates, UQIC_Reg94642 (1ð̊, UQIC); Bellmore Falls, -34.633958 150.555914,

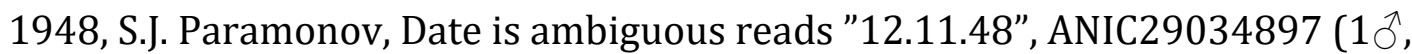
ANIC); Bilpin Blue Mountains, -33.500261 150.532989, 1978, N.W. Rodd, 
AustralianMuseumK408019 (10^, AM); Burralow Swamp, -33.548342 150.592047, 24.ii.1978, N.W. Rodd, AustralianMuseumK410170 (10^, AM); Durras Bay, 35.645003 150.291439, 12.xi.1953, F.M. Hull, UQIC_Reg94722; CNC_Diptera254978-254979; CNC_Diptera254974-254975 (3 $\overbrace{}^{\Uparrow}, 2$ 우, UQIC; CNC); Mannus, near Tumbarumba, -35.808336 147.935786, 1.xii.1950, T.G. Campbell, ANIC29034891 (1ㅇ, ANIC); Mooney Mooney Ck nr. Bosford, -33.437894 151.246467, 25.xi.1975, G. Daniels, G. Daniels Collection, GDCBReg_19721 (1의, GDCB); Mount Tomah, Blue Mountains, -33.549614 150.416033, 28.xi.1977, 2627.x.1978, 25.i.1979, 17.xii.1982, 13.ii.1984, 20.i.1985, 21.i.1985, 28-29.xii.1985, 7.ii.1987, 3.iv.1987, N.W. Rodd, AustralianMuseumK408011;

AustralianMuseumK408014, AustralianMuseumK410123, AustralianMuseumK410129, AustralianMuseumK410136-410138, AustralianMuseumK410142, AustralianMuseumK410161, AustralianMuseumK410171-410172, AustralianMuseumK410276 $(5 \overbrace{}^{\lambda} \widehat{\delta}, 7$ 우, AM); Tooloom, -28.592394 152.453372, 26.i.1926, UQIC_Reg94665 (19, UQIC);

Queensland: 6km W by N of Paluma, -19.000000 146.090000, 25.ix.1980, D.H. Colless, ANIC29034484 (19, ANIC); Blue Mountain, 0.6 km Southeast, -21.6 148.96, 800 to 1058m, 3-4.x.1999, G. Monteith, C. Burwell, Cook, S. Evans, QM_REG._NO._T223200- T223207 (8웅, QM); Gatton, -27.563583 152.279983, 22.iii.1960, J. Martin, UQIC_Reg94595 (1へ̂̄, UQIC); Scrub Road, Brisbane Forest Park, -27.416667 152.833333, [Latitude]27²5'S [Longitude]15250'E 3-10.x.1997, S. Winterton, N. Power, D. White, Malaise trap, UQIC_Reg94798 (1へ̂, UQIC); Tasmania: Geeveston, -43.166500 146.933397, 28.xii.1914, G.H. Hardy, 
ANIC29034896 (1ㅇ, ANIC); Hobart, -42.882139 147.327194, i.1924, G.H. Hardy, ANIC29034898 (10َ, ANIC); Huon-Picton junction, -43.139689 146.714758, 17.ii.1967, E.F. Riek, ANIC29034893 (19, ANIC); South Bruni [Bruny] Island, 43.488236 147.295425, i.1916, Clarke, ANIC29034899 (1へぇ, ANIC); Warra Long Term Ecological Research Site 170, -43.100000 146.683333, 14.i-12.ii.1998, Malaise trap, UQIC_Reg94611 (19, UQIC); A. Simson, 3266, SAMADatabaseNo.29-003356 (1ð, SAMA); Victoria: Gunyah, -38.525603 146.255119, iii.1955, Bornemissza, ANIC29034892 (1우, ANIC); Springvale, -37.947175 145.153067, L.B. Thorn, ANIC29034489 (1q, ANIC); Tarra Bulga National Park, Tarra Bulga Visitors Centre, 38.429578 146.566172, 27.i.2006, W. van Steenis, Jeff_Skevington_Specimen43828 (1ㅇ, UQIC); Barrington Area, Mt. William, -40.934189 148.235269, 152m, 6.iii.1951, B. McMillan, UQIC_Reg94667 (1ㅇ, GDCB); F.M. Hull, CNC_Diptera254976 (1ð , CNC).

Psilota austropsilota Young and Thompson sp. nov.

(Species plate: Fig. 11, Genitalia: Fig. 37C+D, Map: 6)

Body length: $9.2-12.7 \mathrm{~mm}$.

Diagnosis: Face bare. Hind leg simple. Anepisternum, anepimeron, legs, tergite 1 and tergite 2 all red-orange. Tergites 3 and 4 purplish-blue, tergite 3 with a distinct median tubercle. Wing with apical half dark brown. 
Description: Head: Head black, face densely pale pollinose. Face bare of pilosity, frons and vertex pale pilose. Antenna entirely brown, postpedicel somewhat elongate, approximately 2 times as long as wide. Eye sparsely pale pilose.

Thorax: Scutellum, meron, and ventral 3/4ths of katepisternum purplish-black, remainder of thorax red-orange. Scutum mostly purplish-black, with red-orange lateral stripes reaching as far back as the transverse suture. Femora pinkish red, tibiae and tarsomeres orange-brown. Hind leg simple, with no pronounced ridge on femur or tibia. Pleuron shining, free of pollinosity. Thoracic pile white. Wing with apical half of cell $R_{1}$, and all of cells $R_{2+3}$ and $R_{4+5}$ dark brown, with apical $1 / 2$ of cell bm, apical $1 / 2$ of cell cua, and basal $1 / 3^{\text {rd }}$ of cell c bare. Alula microtrichose. Halter pale yellow. Calypter pale with a dark brown border.

Abdomen: Tergite 1 and anterior half of tergite 2 pinkish red. Posterior half of tergite 2 and all of tergites 3 and 4 metallic purple. Posterior corners of tergite 2 and anterior corners of tergite 3 pale yellow. Tergite 3 with a distinct median tubercle. Abdomen white pilose. Sternites 1 and 2 pinkish red, sternites 3 and 4 purple.

Genitalia: Epandrium elongate, about 1.5 times as long as tall. Cerci elongate, approximately 5 times as long as wide. Outer lobe of surstylus inserted amove midheight of inner lobe, longer than inner lobe, and curved slightly downwards on 
apical $1 / 3^{\text {rd }}$. Inner lobe of surstylus reduced, hook shaped, slightly shorter than outer lobe, and tapering smoothly to a downwards-facing point. Phallapodeme smooth. Gonostylus elongate.

Discussion: This species possesses several characters unusual for Psilota, including the hook shaped inner lobe of the male surstylus, the tubercle on tergite 3 , and the bare face. It may represent a separate genus, but should be retained within Psilota until a phylogenetic analysis is completed to test its placement.

Etymology: austro- is a reference to Australia, and the name austropsilota is in reference to the fact that this bizarre species of Psilota is endemic to the country.

Specimens Examined: HOLOTYPE Material: AUSTRALIA: Victoria; Otway NP nature walk; -38.790836 143.543058; W. van Steenis; CNC_Diptera255041 (1ð WVSPC). Paratypes: AUSTRALIA. New South Wales: Dorrigo Nat. Park, -30.365542 152.738950, 15.x.1966, E. Britton, USNM_ENT_00249820 (1 1 , USNM); Mount Tomah, Blue Mountains, -33.539417 150.421811, 2.xii.1994, N.W. Rodd, N.W. Rodd Collection, Jeff_Skevington_Specimen45797; Jeff_Skevington_Specimen45798

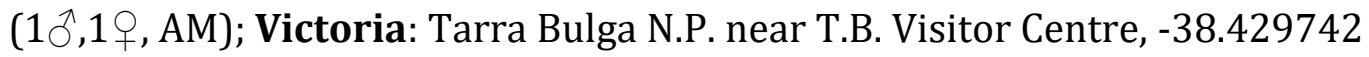
146.566047, 27.i.2006, W. van Steenis, CNC_Diptera253729, CNC_Diptera255040 (2ㅇ, WVSPC); Gippold(??), ii.1896, USNM_ENT249819 (10ึ, 


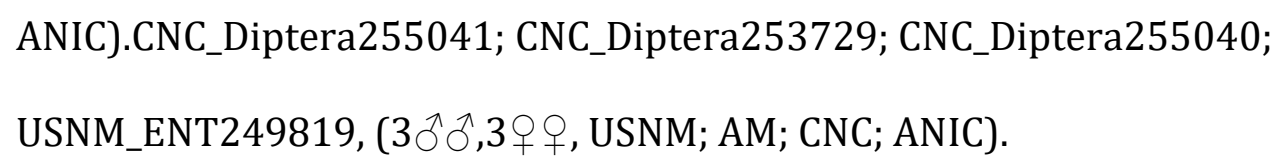

Psilota azurea Young and Thompson sp. nov.

(Species plate: Fig. 12, Genitalia: Fig. 37E+F, Map: 7)

Body length: 8.4-11.0 mm

Diagnosis: Antenna entirely bright orange, thorax and abdomen almost entirely black pilose. Thorax dark metallic and dark blue, abdomen metallic indigo on tergites 3 and 4 . Hind leg simple. Halter and calypter dark.

Description: Head: Face black, with a metallic green sheen. Face sparsely yellow pollinose. Face with yellow pile, frons and vertex with black pile. Antenna entirely bright orange. Eye densely pilose, pile entirely yellow.

Thorax: Thorax dark metallic blue. Hind femur unmodified, with only a few weak, black setulae apicoventrally. Femora mostly dark, with fore and mid femora obscurely dull orange to brown on apical half. Thorax entirely black pilose. Wing faintly brown tinted, entirely microtrichose. Halter dark brown. Calypter brown. 
Abdomen: Tergites 1 and 2 dark metallic blue, tergites 3 and 4 metallic indigo blue. Tergites 2 and 3 entirely black pilose, tergite 4 mostly black pilose with some mixed white pilosity. All sternites dark metallic blue, white pilose.

Genitalia: Epandrium compact, about as long as tall. Cercus approximately triangular. Outer lobe of surstylus inserted near top of inner lobe, smoothly curved downwards at its midpoint. Inner lobe of surstylus narrow, approximately 4.5 times as long as wide, swollen at base, somewhat abruptly constricted at $2 / 3^{\text {rds }}$ its total length, and abruptly swollen and rounded at apex, with the ventral margin curved downwards. Inner lobe of surstylus also with a medially projecting bulge at $2 / 3^{\text {rds }}$ its total length, and upper margin of the tip of the inner lobe curved medially. Phallapodeme with a small ventral tooth. Gonostylus not darkened.

Discussion: Psilota azurea has been collected on individuals of Leptospermum minutifolium and at a light trap.

Etymology: azurea derives from the Latin "azureus", which means "a deep blue colour". This is in reference to the distinctive indigo blue abdomen of Psilota azurea.

\section{Specimens Examined: HOLOTYPE Material: AUSTRALIA: New South Wales;}

Bald Rock N.P.; -28.860783 152.055686; on Leptospermum minutifolium blossom, 6- 
7.XI.1984; D.K. Yeates; UQIC_Reg94741; (1へへ, UQIC). Paratypes: AUSTRALIA. Australian Capital Territory: Black Mtn., -35.274519 149.096908, 25.xi.1959, J.F.B. Common, light trap, CNC_Diptera255027 (1 ${ }^{\curvearrowright}$, ANIC); Blundell's, -35.292886 149.141800, 24.x.1950, Paramonov, USNM_ENT_00249841 (1 1 §, USNM); New South Wales: 20 miles South of Milton, -35.319178 150.439158, 30.ix.1952, S.J. Paramonov, ANIC29034933 (1ㅇ, ANIC); 4 miles North of Bateman's Bay, 35.658458 150.177744, 14.x.1952, S.J. Paramonov, ANIC29034934 (1ㅇ, ANIC); 6.5 miles North of Bateman's Bay, -35.658458 150.177744, 14.x.1959, Z. Liepa, ANIC29034974 (1乞 , ANIC); Grondera Ridge, Royal National Park, -34.114342 151.046586, 18.xi.1978, G. Daniels, GDCBReg_19681 (10^, GDCB); Nadgee Reserve, Merrika River Crossing, -37.306786 149.926247, 13-16.x.1986, E.A. Sugden, Jeff_Skevington_Specimen43827 (1ð̂, QM); Woy Woy, -33.489839 151.319942, 22.ix.1923, Mackerras, ANIC29034973 (1ð, ANIC); Queensland: Mount Moffatt National Park, West Branch campsite, -24.976000 148.010000, 760m, along flowing creek, 23-27.ix.2013, S. Wright, Malaise trap, CNC457413 (1우, QM); Stanthorpe, $28.657928151 .930344,1924,3 . x .1924$, 7.x.1928, Date is ambiguous reads "3.10.24", UQIC_Reg94803, UQIC_Reg94693, CNC_Diptera253728, ANIC29034975 (1 ex, $2 \hat{\jmath}, 3$ 우우 QM, CNC, ANIC).

Psilota basalis (Walker, 1858)

(Species plate: Fig. 13, Genitalia: Fig. 37G+H, Map: 8) 
Body length: 7.8-9.9 mm.

\author{
Diagnosis: Face shining, with pollinosity restricted to two lateral triangles \\ below antenna. Pleuron shining, free of pollinosity. Pile on anepimeron black, \\ pile on remainder of pleuron white. Wing with an anterior brown vitta \\ starting at stigma and ending in cell $R_{4+5}$. Hind leg simple. Tergite 2 with two \\ yellow triangular maculae. Tergite 2 to 4 with posterior margin faintly \\ metallic blue.
}

Description: Head: Head black, face shining blueish-black, with pollinosity only in two lateral triangles below antennae. Face white pilose, frons with mixed black and white pili, vertex black pilose. Scape and pedicel orange, postpedicel dark brown with ventral half orange, oval, approximately twice as long as wide. Eye densely brown pilose.

Thorax: Thorax black. Legs mostly black, with extreme apices of fore- and mid femora orange, basal half of fore and mid tibia orange, and first and second more and mid tarsomeres orange. Hind leg entirely black. Hind femur unmodified, with only a few weak, black setulae apicoventrally. Pleuron shining, free of pollinosity. Pile on anepimeron black, pile on remainder of pleuron white. Pile on scutum and scutellum mostly black, with a few white pili posterior to the transverse suture on the scutum. Wing with an anterior brown vitta starting at the stigma and continuing 
posteriorly through the anterior $1 / 3^{\text {rd }}$ of cell $R_{1}$, the middle of cell $R_{2+3}$, and ending in the middle of cell $\mathrm{R}_{4+5}$. Cell bm entirely bare, cell cua with a few scattered microtrichia apically, cell br mostly bare, with central crease microtrichose, cell c bare on basal $1 / 3^{\text {rd }}$, cells $r_{1}, r_{2+3}$, and $r_{4+5}$ bare basally. Alula bare on basal half. Halter pale yellow. Calypter pale yellow.

Abdomen: Abdomen mostly black. Tergites 2 with two triangular yellow macula, ranging from half the length of the tergite to almost the entire length of the tergite. Tergites 2 to 4 with the posterior rim faintly metallic blue. Tergites shining, free of pollinosity. Lateral sides of tergites 1 and 2, maculae on tergite 2, and apical half of tergite 4 pale pilose, otherwise tergites black pilose. Sternite 2 yellow, other sternites black. All sternites pale pilose.

Genitalia: Epandrium very compact, about 1.5 times as tall as long. Cercus blunt, semicircular. Outer lobe of surstylus inserted at top of inner lobe, thick and curved smoothly downwards over its entire length. Inner lobe of surstylus short, swollen at base, sharply constricted at midlength, approximately 3.5 times as long as wide (at base), and rounded at apex. Inner lobe of surstylus also with a medial projection on the dorsal half of the inner surface running the length of the surstylus. Tip of the inner lobe of surstylus curved medially on the dorsal surface, forming a second medial projection. Aedeagal apodeme with a downwards facing projection on the ventral surface. Gonopods elongate, extending well beyond the tip of the phallus. 
Discussion: This species was originally described from a female specimen collected in the Aru Islands Regency (Maluku Province, Indonesia). Thus, this represents the first description of the male of this species and the first record of this species in Australia. This is assumed to be the same species due to the distinct brown vitta on the wing and the faint metallic blue markings on the abdomen, which match the holotype very well.

Specimens Examined: HOLOTYPE Material: INDONESIA: Maluku Province: Aru. Jeff_Skevington_Specimen_45632. (19, BMNH). Other material: AUSTRALIA: Queensland: $14 \mathrm{~km} \mathrm{~W}$ by N of Hope Vale Missn., -15.160000 144.590000, at light, 8.X.1980;9.V.1981, coll.D.H. Colless, USNM_ENT_00249846-00249847 (2ðð

Psilota bicolor Young and Ferguson sp. nov. (Species plate: Fig. 14, Genitalia: Fig. 38A+B, Map: 9)

Body length: 7.3-12.1 mm.

Diagnosis: Face black. Hind leg simple. Abdominal tergites 1, 3 and 4 black, tergite 2 yellow, usually with posterior margin black. 
Description: Head: Frons and sides of face black, densely white pollinose, face with a broad shining median stripe running from below the antennal base to the oral margin. Face and frons with white hairs, vertex with a tuft of long black hairs. Antenna orange. Eye densely haired, hairs entirely white.

Thorax: Fore femur and fore tibia mostly orange, each with an obscure, posterior dark stripe towards their apex. Mid femur mostly dark, with a thin, anterior orange stripe near the base. Mid tibia orange. Hind femur entirely dark, with two rows of strong dark setula, ventral carinate ridge absent. First tarsomere on all legs orange with a thin dark anterior margin, all other tarsomeres dark. Thorax dark brown to black, entirely shining. Pile on pleuron entirely white, metepimeron and katepimeron bare. Scutum with pile entirely white anterior to transverse suture, pile mostly dark behind transverse suture. Scutellum with pile mostly white, but with some dark pile interspersed evenly. Wing colourless, with bare areas on the anterior half of cell c and anterior quarter of cell bm. Halter pale.

Abdomen: Tergites 1, 3 and 4 almost entirely dark, tergites 1 and 3 sometimes with very narrowly yellow posterior/anterior margins respectively. Tergite 2 entirely yellow except for a narrow dark band on the posterior margin, not reaching the edges of the tergite. Sternites 1, 3 and 4 entirely dark. Sternite 2 entirely yellow. 
Genitalia: Epandrium compact, about as long as tall. Cercus approximately triangular, slightly elongate. Outer lobe of surstylus inserted at top of inner lobe, thin and curved gradually downwards over its entire length, slightly broader and more strongly curved towards the apex. Inner lobe of surstylus straight, slightly narrowed at midlength, and smoothly rounded at apex. Phallapodeme with a small, forward facing ventral projection. Gonostylus thick, not darkened.

Discussion: Nothing is known about the biology of this species.

Etymology: Psilota bicolor appears to be a Ferguson manuscript name that has spread throughout Australian insect collections. It is here being published as a valid name for the first time, and describes the bicolorous abdomen of this species.

\section{Specimens Examined: HOLOTYPE Material: AUSTRALIA: New South Wales;}

Warrumbungle National Park; -31.279689 149.046797; Malaise trap; 19.X3.XI.1997; J. Skevington, S. Winterton; UQIC_Reg94800; (1 $\overbrace{}^{\lambda}$, UQIC). Paratypes:

AUSTRALIA. New South Wales: $10 \mathrm{~km}$ West of Murwillumbah, -28.331308 153.281278, 25-27.ix.1981, N.W. Rodd, AustralianMuseumK409930 (1ð , AM); 3 km SW of Sandy Hollow, -32.368508 150.566889, 6.xii.1976, G. Daniels, GDCBReg_19688 (1へ̂, GDCB); Goondiwindi, -28.539150 150.298089, ix.1935, G.H. Hardy, AustralianMuseumK407106 (1ðึ, AM); Mona Vale, -33.675961 151.306533, 


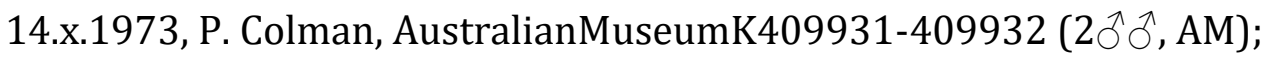

Queensland: 30 mi. W Collinsville, -20.578364 147.374953, 12.ix.1950, E.F. Riek, USNM_ENT249845 (1ðð, ANIC); Brisbane, Mt. Coot-tha, -27.483333 152.95, [Latitude]27²9'S [Longitude]15257'E, 170m, Hilltopping, 10.viii.1997, J. \& A.

Skevington \& S. Winterton, UQIC_Reg94801 (1ð, UQIC); Brisbane, -27.526975

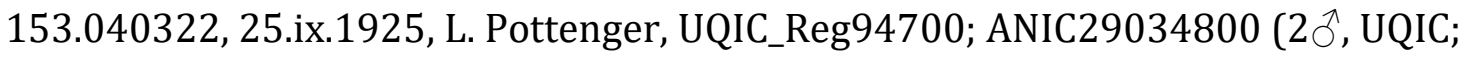
ANIC); Eungella 45 miles West of Mackay, -21.128750 148.488333, 426 to $731 \mathrm{~m}$, 13-25.ix.1923, Goldtiner, Collector name is unclear, ANIC29034799 (1 $\overbrace{}^{\wedge}$, ANIC); Lamington NP, IBISCA, -28.638000 153.121000, 23.i-3.ii.2009, G. Monteith \& F. turco, Malaise trap, QM_REG._NO._T222147-222148 (2우, QM); 6-22.i.2009, G. Monteith, Malaise trap, QM_REG._NO._T222146 (19+, QM); One Tree Hill, 1 km East, 25.283333 151.916667, 180m, 26.ix.1999, S.G. Evans, QM_REG._NO._T223228 (19, QM); Stockyard Creek, SE of Capalaba, -27.550000 153.166667, 24.ix.1985, G. and A. Daniels, GDCBReg_19563 (1§ึ, GDCB); Yarraman, 2.9km SSE, -26.865000 151.995000, 12-28.iii.2010, G. Monteith, Malaise trapQM_REG._NO._T222149 (1우, QM); “Chilla”, 13.iv.1929, Aust. Nat. Ins. Coll., ANIC29034798 (10̄, ANIC).

\section{Psilota brunnipennis Young sp. nov.}

(Species plate: Fig. 15, Genitalia: Fig. 38C+D, Map: 10)

Body length: $16.0-16.5 \mathrm{~mm}$. 


\section{Diagnosis: Large, black species with apex of wing darkened, distinguishing it}

from all other Australian Psilota. Hind femur with a low apicoventral setulose carina. Hind tibia simple. Male genitalia slightly asymmetrical, with a small downwards facing hook on the left outer surstylar lobe.

Description: Head: Head black, face densely pale pollinose. Face pale yellow pilose, frons mostly black pilose with a few pale pili, vertex black pilose. Antenna entirely black, postpedicel somewhat elongate, approximately 2.5 times as long as wide. Eye densely brown pilose.

Thorax: Thorax black. Legs mostly black, with apices of femora and fore- and mid tibiae brown. Hind femur with a low apicoventral setulose carina. Hind tibia simple, with no basoventral spur. Pleuron pale yellow pollinose. Pile on pleuron and scutellum entirely pale, pile on scutum mixed black and pale yellow. Wing with apical tip dark brown, with apical $3 / 4^{\text {ths }}$ of cell $\mathrm{bm}$, apical $1 / 4^{\text {th }}$ of cell cua, and a thin apical strip of anal lobe bare. Alula mostly bare, with some scattered microtrichia near base and apical edge. Halter orange. Calypter pale with a dark brown border.

Abdomen: Abdomen black. Tergite 1 pale pilose. Tergite 2 mostly pale yellow pilose with some black pile on posterior half. Tergite 3 mostly black pilose with pale yellow pile only on lateral edges. Tergite 4 entirely black pilose. Sternites shining, mixed black and pale yellow pilose. 
Genitalia: Epandrium compact, about as long as tall. Cercus oval, somewhat elongate. Outer lobe of surstylus inserted at top of inner lobe, curved smoothly downwards on apical half, with a small tooth on the ventral surface. Inner lobe of surstylus long and straight, approximately 5 times as long as wide (at base), slightly narrowed at midlength, and smoothly rounded at apex. Phallapodeme with a small, forward facing spur on ventral side. Gonostylus darkened.

Discussion: Nothing is known about the biology of Psilota brunnipennis.

Etymology: bruni- derives from the Latin brunius, for "brown", and pennis derives from the Latin penna, for "wing". This is in reference to the brown wingtips of Psilota brunnipennis, which easily distinguish it from all other species.

Specimens Examined: HOLOTYPE Material: South Black Range, east of Hoskinstown, 35.411111 149.534444, 1130mm, ANIC29035775, 11.IX.2003, D.J. Ferguson, $(10$, ANIC). AUSTRALIA: New South Wales: AUSTRALIA: New South Wales: Bilpin, Blue Mountains; -33.500261 150.532989; -26.IX.1978; coll.N.W. Rodd; AustralianMuseumK411054; (1ๆ AM). 


\section{Psilota calva Young sp. nov.}

(Species plate: Fig. 16, Genitalia: Fig. 38E+F, Map: 11)

Body length: $7.8 \mathrm{~mm}$.

Diagnosis: Face shining, with pollinosity restricted to two lateral triangles below antenna. Pleuron shining, free of pollinosity. Pile on anepimeron black, pile on remainder of pleuron white. Hind leg simple. Wing colourless. Halter pale yellow. Tergite 2 unmarked. Tergite 2 to 4 with posterior margin faintly metallic blue.

Description: Very similar to Psilota basalis, differing as follows:

Head: Eye white pilose.

Thorax: Thorax black. Legs mostly black, with extreme apices of fore- and mid femora orange, basal half of fore and mid tibia orange. Hind leg entirely black. Pile on anepimeron black, pile on remainder of pleuron white. Pile on scutum mostly white, with black pili mixed in on posterior half. Scutellum with mixed black and white pile. Wing colourless. Wing more extensively bare, with only scattered 
microtrichia in the apical $1 / 3^{\text {rd }}$ of cells $\mathrm{r}_{4+5}, \mathrm{dm}$, and cua. Cell $\mathrm{m}_{4}$ mostly microtrichose. Calypter white.

Abdomen: Tergite 2 unmarked. Tergite 2 mostly white pilose, with only posterior margin black pilose. Sternites black.

Genitalia: Cercus triangular, somewhat elongate. Inner lobe of surstylus more strongly constricted at midlength. Medial projections on inner lobe of surstylus very narrow, projecting less than in P. basalis. Aedeagal apodeme smooth.

Discussion: Nothing is known about the biology of this species.

Etymology: calva derives from the Latin calvus, meaning "bald, or bare". This is in reference to the bare, shining face of Psilota calva.

Specimens Examined: HOLOTYPE Material: AUSTRALIA: Northern Territory:

Brock's Creek, Burnside, N. Aust., 23.IV.1929, -13.466557 131.415973, coll.T.G. Campbell, USNM_ENT_00249849 (10̂ USNM). 


\section{Psilota coerulea Macquart, 1846}

(Species plate: Fig. 17, Genitalia: Fig. 38G+H, Map: 12)

Psilota coerulea Macquart, 1846: 268. Type locality: Tasmania, Australia.

Merodon muscaeformis Walker, 1852: 244. Type locality: Tasmania, Australia.

Body Length: 9.4-14.0 mm.

Diagnosis: Metallic blue species with frons pale pilose, pleuron shiny and black pilose. Hind femur with a distinct rounded apicoventral setulose carina. Hind tibia simple. Calypter entirely brown. Tergite 4 white pilose. The black pilose pleuron distinguishes $P$. coerulea from similar species like $P$. aislinni, $P$. alexanderi, P. darwini, P. pollinosa, and P. viridis.

Description: Head: Head black, face densely pale pollinose. Face and frons yellow pilose, vertex black pilose. Antenna usually entirely orange, rarely dull brown dorsally on postpedicel. Eye densely yellow pilose.

Thorax: Thorax dark, metallic blue. Legs mostly dark brown, with apices of femora, and entire fore and mid tibia sometimes pale brown/orange. Hind femur with a distinct rounded apicoventral setulose carina. Hind tibia simple, with no basoventral spur. Pleuron shining, pollinosity free. Pile on pleuron, scutum, and scutellum 
entirely black. Wing colourless, with cells c, r, bm, cua, and the anal lobe mostly bare. Cells $\mathrm{r}_{4+5}, \mathrm{r}_{2+3}$, and dm with bare areas basally. Alula completely microtrichose. Halter dark brown. Calypter pale brown to dark brown, always with a dark brown border.

\begin{abstract}
Abdomen: Abdomen metallic blue/green. Tergites 2 and 3 with white pile on basal half and black pile on apical half, tergite 4 entirely white pilose. Sternites shining, white pilose.
\end{abstract}

Genitalia: Epandrium compact, about as long as tall. Cercus approximately triangular. Outer lobe of surstylus inserted just above mid-height of inner lobe, curved downwards sharply at $1 / 3^{\text {rd }}$ its length. Inner lobe of surstylus strongly projected ventrally at base forming a triangular plate, slightly contricted at midlength, then broadening slightly towards a rounded apex. Phallapodeme with a small ventral projection. Gonostylus thick, darkened.

Discussion: The holotype specimen for this species is very badly damaged by dermestid beetles, making confident identification difficult. However, this is the most widespread and commonly collected metallic blue species, and what is left of the specimen does conform to the general habitus and pile colouration described 
here. Therefore, the name coerulea can be associated with this morphotype with some degree of certainty.

\section{Specimens Examined: HOLOTYPE Material (Psilota coerulea): AUSTRALIA:}

Tasmania. Bears labels reading "HOLOTYPE”, “MNHN, Paris ED9367”, “13 44”, “Psilota coerulea n. sp. Macq.". (1 $\overbrace{}^{\Uparrow}$ MNHN, photograph). HOLOTYPE Material (Psilota muscaeformis): AUSTRALIA: Tasmania. Bears label reading "VDL", “HOLOTYPE FEMALE OF Merodon muscaeformis Wlk. VAN DIEMEN'S LAND”, J.

Skevington Specimen_45634, (1乞ึ BMNH, photograph). Other material: AUSTRALIA. Australian Capital Territory: Black Mountain, -35.273675 149.097583, 25.ii.1957, I.F.B. Common, light trap, ANIC29034817 (1, ANIC); Mount Ainslie, -35.266667 149.166667, 28.xi.1996, C.J. Burwell, QM_REG._NO._T223174 (1ðð, QM); New South Wales: 24 km W South Grafton, -29.616667 152.733333, 19.ix.1981; 8.ix.1983; 3.x.1985; 26.ix.1986; 29.ix.1986; 29.ix.1989, R. Eastwood, GDCBReg_19565; GDCBReg_19667-19668; GDCBReg_19694-19695; GDCBReg_19697; GDCBReg_19774; UQIC_Reg94590; UQIC_Reg94707 (9ðð GDCB, QM); 6 km Northeast Bilpin, Blue Mountains, -33.462750 150.572836, 28.xi.1987, N.W. Rodd, AustralianMuseumK406923 (1ㅇ, AM); Congo, -35.959786 150.135839, 3.x.1981, M.S. Upton, ANIC29034820 (1q, ANIC); Gosford, -33.426686 151.341658, x.1924, ANIC29034815 (1우, ANIC); Haystack Ridge, Blue Mountains, -32.914211 149.964019, 19.xii.1985, N.W. Rodd, AustralianMuseumK406921 (1ㅇ, AM); Jenolan Caves, -33.819039 150.022917, 609m, ANIC29034458 (1우, ANIC); Katoomba, - 
33.713133 150.313267, 18.ii.1954, AustralianMuseumK410150 (1, AM); Mount Tomah, Blue Mountains, -33.549614 150.416033, 29.ix.1977; 18.x.1980, N.W. Rodd, AustralianMuseumK407026; AustralianMuseumK410291 (1○1우, AM); Narrabeen, 33.719683 151.297125, 16.ix.1934, D.F.W., ANIC29034814 (1§^, ANIC); Sydney, $33.926375151 .125936,1925$, Date is ambiguous reads "2.1.25", ANIC29034818 (1ㅇ, ANIC); Queensland: Carnarvon N.P., Summit Of "Fly Hill”, near West Branch Camp, -24.974167 147.992778, 900m, 12.x.2002, J. Skevington; J.E. O'Hara, CNC_Diptera254989-254990; CNC_Diptera254994-254997 (5つ̋ð, CNC); Kenniff's Lookout, Mt Moffatt sect., Carnarvon Nat Pk, -25.165167 147.840419, 25.ix.1986, D.K. Yeates, UQIC_Reg94661 (1へ̂, UQIC); Kuranda, -16.816878 145.633497, ANIC29034453 (1ㅇ, ANIC); Mount Moffat National Park, Mount Rugged summit, 24.900000 148.000000,1130m, 24.xi.1995, C.J. Burwell, QM_REG._NO._T223173 $(1 \delta$, QM); Mount Moffatt, Mt Moffatt sect., Carnarvon Nat. Pk., -25.165167 147.840419, 26.ix.1986, D.K. Yeates, UQIC_Reg94660 (10^, UQIC); Mount Tamborine, -27.923058 153.171667, 1923, W.H. Davidson, QM_REG._NO._T223175 (19, QM); Near Rosedale, -24.631128 151.913664, 27.x.1974, H. Frauca, ANIC29034916 (1ð, ANIC); Tasmania: Mole Creek, -41.558628 146.404517, 4.ii.1910, ANIC29034457 (1, ANIC); Mount William National Park, Summit of Mount William, -40.909722 148.186389, 23.xii.1998, J. \& A. Skevington, UQIC_Reg94553-94554 (2ððð, QM); Swansea, -42.134133 148.058614, Lea, ANIC29034808; ANIC29034816 (2우우, ANIC); Victoria: Beaconfield, -38.048778 145.371733, A.M. Wade, ANIC29034821 (19, ANIC); Broadwater National Park, -29.050325 153.427539, 6.ix.1981, D. Yeates, U.Q.I.C. Loan \#1036, UQIC_Reg94582 (1§, QM). 


\section{Psilota cupreus Macquart, 1850}

(Species plate: Fig. 18, Map: 13)

Psilota cupreus Macquart, 1850: 317. Type locality: Tasmania, Australia.

Body length: $6.8-11.4 \mathrm{~mm}$.

Diagnosis: Face black, pale pollinose, with pale pile. Frons and vertex black pilose. Thorax black. Thoracic pile white, or mixed black and white. Hind leg simple. Calypter variable in colour. Halter variable in colour. Abdominal tergites 2 to 4 mostly to entirely red. Tergite pile variable in colour.

Description: Head: Head black, face white pollinose. Face white, frons and vertex black pilose. Antenna orange to dark brown. Postpedicel variable in shape. Eye densely brown pilose.

Thorax: Thorax black. Legs entirely orange to entirely dark brown. Hind femur simple. Hind tibia simple. Pleuron lightly white pollinose or bare and shining. Pile pleuron entirely white or with mixed black and white pile. Pile on scutum and scutellum entirely white or with mixe black and white pile. Wing colourless. Wing 
microtrichia variable. Alula completely microtrichose. Halter variable in colour. Calypter variable in colour.

Abdomen: Tergite 1 black. Tergite 2 entirely red or with a large, anterior black area. Tergite 3 entirely red or with a small medial black spot. Tergite 4 entirely red. Pilosity of tergites variable in colour. Sternites 2 to 4 reddish, with variably coloured pilosity.

Genitalia: variable (see below).

Discussion: Psilota cupreus doubtlessly encompasses multiple species as described here. Initial DNA barcode analysis indicates that at least six species are represented (Figure 43). Further DNA barcoding and male genitalia dissection are required to adequately separate the full range of specimens into species, and will be tackled in a future revision. Furthermore, there are several other species of Psilota described from Australia that may or may not be synonyms of $P$. cupreus, including $P$. erythrogaster, P. rubra, and P. rubriventris (See species not recognized below). Because it is impossible to tell what name should properly be assigned to different barcode clusters without a full revision of this complex, these species have not been treated individually. 
Specimens Examined: AUSTRALIA. Australian Capital Territory: Black

Mountain, -35.273675 149.097583, 21.x.1966; 23.x.1969, I.F.B. Common, light trap, ANIC29034958; ANIC29034939; CNC_Diptera253814 (3우, ANIC); Blundell's [Cabin], -35.292886 149.141800, 20.i.1935; 11.x.1950; 24.x.1950, S.J. Paramonov; Fuller, ANIC29034941; ANIC29034951; ANIC29034953-29034954; CNC_Diptera253704; CNC_Diptera253804 (2ððð,3우우, ANIC); Blundells Ck., 35.220000 148.500000, ii.1987, D.H. Colless, Malaise trap, CNC_Diptera253794 (1우, ANIC); Canberra - CSIRO Botanical Garden, -35.278878 149.108917, 26.x.1988, F.C. Thompson, USNM_ENT_00034137; USNM_ENT_00034139-00034141 (4우, USNM); Canberra, Terr. 1, -35.282000 149.128686, 20-30.xi.1953, M. Hull, CNC_Diptera254972 (10ึ, CNC); Canberra, -35.282000 149.128686, 2.xi.1947, S.J. Paramonov, ANIC29034940 (10^, ANIC); Lees Creek Brindabella Rge., -35.338461 148.867925, 1.i.1975, G. Daniels, GDCBReg_19737 (1ㅇ, GDCB); Mount Ainslie, 35.266667 149.166667, 28.xi.1996, C.J. Burwell, QM_REG._NO._T223209 (1ठ̂, QM); New South Wales: 'Lugen Park' Nullo Mountain Northeast of Rylstone, -32.649278 150.217650, 22.x.1953, T.G. Campbell, ANIC29034942 (1 $\widehat{\partial}^{\lambda}$ ANIC); 20 miles South of Milton, -35.319178 150.439158, 30.ix.1952, S.J. Paramonov, ANIC29034943; ANIC29034959; CNC_Diptera253706; CNC_Diptera253815 (4우우, ANIC); 3 km North of Lansdowne via Taree, -31.758753 152.534258, on house steps, wet sclerophyll forest, rainforest margin, 25.xii.1988, G. Williams, AustralianMuseumK410625 (1ㅇ, AM); 3 km South of Mount Wilson, Blue Mountains, -33.516011 150.378642, 29.xi.1984, D.K. McAlpine, B.J. Day,

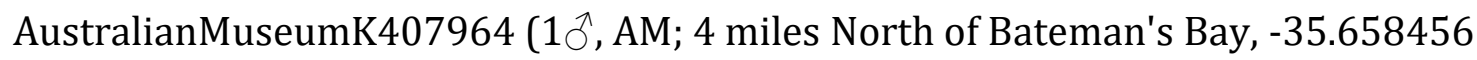


150.177744, 14.x.1952; 12.x.1956; 22.x.1952, S.J. Paramonov, ANIC29034956; ANIC29034950; CNC_Diptera253705; CNC_Diptera253709 (1〕3우우, ANIC); 4 mls W. of Amosfield, -28.658686 152.016650, 17.x.1971, E.M. Exley, UQIC_Reg94770 (1우, UQIC); 6 km Northeast Bilpin, Blue Mountains , -33.462750 150.572836, 23.ix.1992, N.W. Rodd, AustralianMuseumK410278 (1ㅇ, AM); Back Yamma State Forest, -33.339906 148.223703, 11.xi.1964, D.H. Colless, CNC_Diptera253708 (1우, ANIC); Bald Rock N.P., -28.8525 152.055556; -28.852500 152.055558, on Leptospermum minutifolium blossom, 6-7.xi.1984, D.K. Yeates, UQIC_Reg94630; UQIC_Reg94631-94633; UQIC_Reg94637-94638; UQIC_Reg94744-UQIC94745; UQIC_Reg94675 (4ふðぇ5ㅇ, UQIC); Bawley Point, -35.500000 150.400000, 2123.ix.2000, Rentz, CNC_Diptera253696 (10 , ANIC); Bilpin, Blue Mountains , 33.500259 150.532989, 21.ix.1978, N.W. Rodd, AustralianMuseumK410429 (1우, AM); Blackheath, -33.635539 150.283625, 24.xii.1921, CNC_Diptera253806 (1우, ANIC); Boonoo Boonoo Falls, -28.801247 152.165061, 12.xi.1981, G. \& A. Daniels, GDCBReg_19687 (1ㅇ, GDCB); Cherrybrook, electricity tower access track, end of Trevor's Lane, -33.710139 151.059500, 13.ix.2008, S. Ginn, AustralianMuseumK410146 (1ð, AM); Clyde Mountain, eastern foothills, 35.546072 149.950333, 1219m, ex brackett fungus: Stereum semilugens, 15.viii.1967, Z. Liepa, Emerged 25.VIII.1967, ANIC29034945; ANIC29034952;

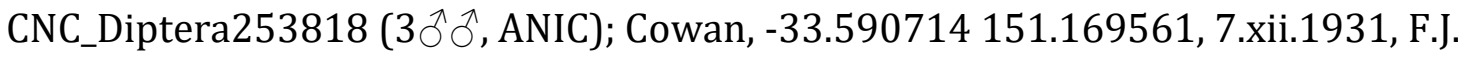
Gay, ANIC29034961 (1ㅇ, ANIC); Dobroyd Point, Sydney, -33.926375 151.125936, 27.xi.1978, N.W. Rodd, AustralianMuseumK410619 (19, AM); Durras Bay, 35.645003 150.291439, 12.xi.1953, G. Daniels, CNC_Diptera253846 (1우, CNC); 
Durras, -35.646775 150.296939, 29.ix.1959, Z. Liepa, ANIC29034969 (1ㅇ, ANIC); Gibraltar Range National Park, Waratah track, -29.5 152.333333, on Leptospermum sp. Blossom, 10-12.xi.1984, D.K. Yeates, UQIC_Reg94634 (1ㅇ, UQIC); Goondera Ridge, Royal National Park, -34.114342 151.046586, 2.x.1978, G. Daniels,

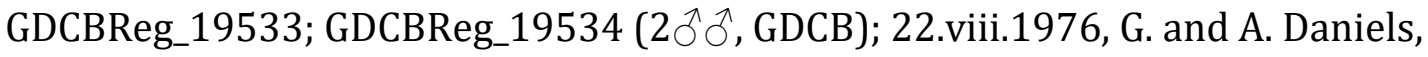
GDCBReg_19535 (1ðð, GDCB); Heathcote NP, Kingfisher Pool, -34.117042 150.952122, dry eucalypt forest, 16.x.1994, B.J.Sinclair, CNC_Diptera106594 (1우, CNC); Jervis Bay, -35.035431 150.668869, 4.ix.1948, E.F. Riek, ANIC29034949 (10^, ANIC); Killara, -33.768639 151.163469, 13.x.1934, D.F.W., ANIC29034955 (1ㅇ, ANIC); Lorien Wildlife Refuge, 3 km North of Lansdowne, -31.758753 152.534258, 7.xi.1993, G. \& T. Williams, AustralianMuseumK411046 (1 $\overbrace{}^{\lambda}$, AM); Mooney Mooney Creek, near Gosford, -33.437894 151.246467, 1.xii.1989, D.K. McAlpine, B.J. Day, AustralianMuseumK408009 (1ㅇ, AM); Mount Canobolas State Recreation Area, summit Mount Canobolas, 12.8 km Southwest of Orange, -33.343889 148.982500, 1395m, 18.x.2002, C. Lambkin, N. Starick, hand collected, ANIC29035762 (1〇^, ANIC); Mount Gibralter, Comboyne Plateau, -31.644328 152.422989, shrub complex, 21.i.1985, G. Williams, AustralianMuseumK410621 (1ㅇ, AM); Mount Tomah, Blue Mountains , -33.549614 150.416033, 26.ix.1978; 26.x.1978; ); 28.xii.1985, N.W. Rodd, N.W. Rodd Collection, AustralianMuseumK410430; AustralianMuseumK410252; AustralianMuseumK410154; AustralianMuseumK410163; AustralianMuseumK410175; AustralianMuseumK410182 (2ðð3우, AM); Mt Canobolas State Recreation Area, summit Mt Canobolas 12.8 km SW Orange, -33.343889 148.982500, 1395m, 
18.x.2002, C.L. Lambkin, N.T. Starick, hand net, CNC_Diptera253847 (1ㅇ, ANIC); Nadgee NR, near Eden, -37.399378 149.908592, wet sclerophyll forest, 21.xii.1985; 14-15.ii.1986, E.A. Sugden; D.J. Bickel, AustralianMuseumK410515 (1오 AM); Nadgee Reserve 4.7km S Merrica Hut, -37.399378 149.908592, 21.xii.1985; 21.xii.1986, E.A. Sugden, CNC_Diptera254983; Jeff_Skevington_Specimen43807; Jeff_Skevington_Specimen43810-43811 (1§3우우, CNC; QM); New England Nat. Pk. Via Ebor, -30.476022 152.660183, 22-23.i.1966, B. Cantrell, UQIC_Reg94705 UQIC_Reg94636; UQIC_Reg94635 (3우, QM); New England Nat. Pk. near Wright's Lookout, -30.476022 152.660183, 14.x.1961; 29-31.x.2002, C.W. Frazier; G.,M.Wood, CNC_Diptera253803; CNC_Diptera106427 (2 + , ANIC; CNC); Pigeon House Mtn., Ulladulla, -35.349175 150.265064, 13.xi.1976, G. Daniels, GDCBReg_19767 (1우, GDCB); Royal National Park, Flat Rock Creek, near Sydney, 33.926375151 .125936 , From the rotten caudex of Xanthorrhoea resinosa, 10.viii.1973, D.K. McAlpine, S.P. Kim, emergence on 4.IX.1973, AustralianMuseumK410255 (1우, AM); Rutherford Ck Brown Mtn. nr. Nimmitabel, 36.601300 149.381311, 26.xii.1974, G. Daniels, GDCBReg_19536 (1へ̂, GDCB); Rutherford Creek, Brown Mtn. nr Nimmitabel, -36.581667 149.551111, 14.xi.1976, G. Daniels, GDCBReg_19684 (1ㅇ, GDCB); Siding Springs Observatory, Summit, 31.255558 149.061111, [Latitude]31¹5'20"S [Longitude]149³'40"E, 17.x.1997, J. Skevington, C. Lambkin, S. Winterton, UQIC_Reg94532-94536 (5ふふふ, QM); Stanwell Tops, -34.219903 150.991808, 9.x.1977, G. Daniels, GDCBReg_19528-19531 (4ð̋, GDCB); Tindery, -35.754172 149.280764, 1.i.1938, E.H. Kipps, ANIC29034968 (1우, ANIC); Wahroonga, Cook Trig, trail off Grosvenor Street, -33.699450 151.126500, 
28.ix.2007, D. Britton, S. Ginn, L. Holme, LHP002/01, AustralianMuseumK410478 $(1 \overbrace{}^{\lambda}, A M) ;$ Warrumbungle National Park Area, -31.272222 149.061111; -31.279689 149.046797, observatory hilltop; Observatory hilltop, 17.x.1997, J. Skevington, UQIC_Reg94543-94545 (3ð̋, UQIC); Waste Point, Kosciusko National Park, 36.427114 148.309022, 24.i.1984, David W. Inouye, AustralianMuseumK410600 (1우, AM); West of Mount Mackenzie, 9 km West of Tenterfield, -29.066667 151.966667, paddock with bracken fern, 9.xii.2003, G. Daniels, AustralianMuseumK411060 (1ㅇ, AM); Woolgoolga, -30.113036 153.183792, 28.i.1923, Health Dept., CNC_Diptera253805 (1q, ANIC); Woy woy, -33.489839 151.319942, 3.x.1925, Mackerras, CNC_Diptera253809 (1+, ANIC); Queensland: 7 km North of Maryborough, -25.470267 152.695200, 23.viii.1977, K.J. Lamkin, QM_REG._NO._T223216 (19, QM); Agnes Water, -33.710139 151.059500, 3.ix.1987, N.W. Rodd, AustralianMuseumK410174 (1ð̊, AM); Bald Rock National Park, Boundary Swamp Creek, -28.816667 152.066667, Veg. over water, 11.x.1998, J. Skevington, sweeping, UQIC_Reg94746 (1今̂, UQIC); Bluff Range, West Slope, Biggenden, -25.600000 152.050000, 21.viii.1975, H. Frauca, ANIC29034946; CNC_Diptera253816 (2 9 , ANIC); Brisbane, -27.526975 153.040322, 1911, H. Hacker, QM_REG._NO._T223208 (10ึ, QM); x.1925, G.H. Hardy, AustralianMuseumK407091 (1ㅇ, AM); Brown Lake, N Stradbroke Is., S.E.Q., 27.490105 153.432379, on Leptospermum flavescens blossom, 21-24.ix.1984, D.K. Yeates, UQIC_Reg94674 (1ð̊, UQIC); Bundaberg, -24.866972 152.350972, viiiix.1971, H. Frauca, ANIC29034970 (19, ANIC); Collaroy, Sydney, -33.926375 151.125936, 1921, Health Dept. Date is ambiguous reads "2.9.21", ANIC29034947 
(1へ̆, ANIC); Dayboro, 0.5 km South, -27.200000 152.816667, 18-19.ix.1999, S.G. Evans, QM_REG._NO._T223227 (1ㅇ, QM); Flinders Peak nr. Boonah, -27.800000 152.816667, summit, 13.ix.1995, D.K. Yeates, UQIC_Reg94538-94539 (2へ⿱人, UQIC); Flinders Peak, via Peak Crossing, -27.816667 152.816667, 13.ix.1995, C.J. Burwell, D.K. Yeates, QM_REG._NO._T223211-223215 (5つึ๊, QM); Girraween National Park, Hilltop 1.2km NE of Bald Rock Campground, -28.821942 151.937850, 31.xii.2014; 1.i.2015, J.H. Skevington, CNC384663-384670; CNC384926-384927; CNC38462438430; CNC451705-451706; CNC451709 (21ððへ, CNC); Kenniff's Lookout, Mt. Moffatt sect., Carnarvon Nat. Pk., -25.165167 147.840419, 25.ix.1986, D.K. Yeates, UQIC_Reg94624 (1ð^, UQIC); Lamington N.P., -28.045967 153.127983, 29.x.1955, F.A. Perkins, UQIC_Reg94905 (1우,UQIC); Miriam Vale, -24.330397 151.557592, on Angrophora costata, 5.xii.1977, T. Sears, UQIC_Reg94623 (1今, UQIC); Mount Marlay Summit, Stanthorpe, -28.666667 151.933333, 20.x.1997; 22.x.2005, J. Skevington, C. Lambkin, S. Winterton; D. K. Yeates, UQIC_Reg94539-94541; ANIC29035763-

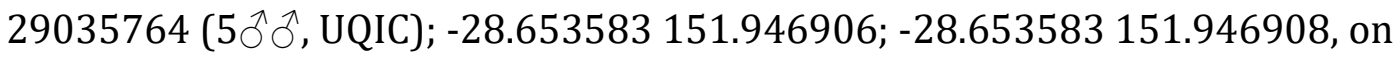
Leptospermum, 22.ix.1985, D.K. Yeates, UQIC_Reg94626; , UQIC_Reg94639; UQIC_Reg94668; UQIC_Reg94735 (4仓̋̂̉, QM); Mt. Beerburrum, Glasshouse Mts., 26.950000 152.933333, 280m, 29.x.1991, R. Eastwood, GDCBReg_19735 (1ð^, UQIC); Mt. Norman Area, Via Wallangarra, -28.873267 151.973822, 2.x.1971, G.B. Monteith, UQIC_Reg94769 (19, UQIC); Near Rosedale, -24.631128 151.913664, 27.x.1974, H. Frauca, ANIC29034948; ANIC29034965 (1へð,1우, ANIC); Six Mile Creek, W of Elimbah, -27.016667 152.933333, 15.ix.1985, G. and A. Daniels, GDCBReg_19569 (1우, UQIC); Stanthorpe, -28.657928 151.930344, 22.ix.1979, S.J. 
Johnson, GDCBReg_19532 (1ð^, GDCB); Summit of Mount Greville, -28.074028 152.502008, 393m, 3.i.2015, J.H., A.M. and A.W. Skevington, CNC451749 (1 $\overbrace{}^{\lambda}$, CNC); Tambourine Mountain, -27.923086 153.171717, 28.x.1912, H. Hacker, QM_REG._NO._T223184 (1+, QM); The Blunder, Brisbane, -27.526975 153.040322, 9.x.1966; 18.ix.1966; 25-26.ix.1966, C.F. Ashby, ANIC29034944; ANIC29034964; ANIC29034966; CNC_Diptera253707 (1 3 3우 , ANIC); Woombye, near Nambour, 26.666911 152.966606, 11-16.x.1965, D.H. Colless, ANIC29034963 (1ㅇ, ANIC); along Rd to 18 Mile Swamp, N. Stradbroke Island, -27.516667 153.483333, 17.ix.1989, M.A. Schneider, UQIC_Reg94771 (1q, UQIC); South Australia: Adelaide Area, Mount Lofty, -34.984972 138.708333, 4.x.1997, J. Skevington, CNC_Diptera254973 (1ð̊, UQIC); Adelaide, Belair National Park, -35.000000 138.633333, Hilltopping, 4.x.1997, J. \& A. Skevington, C. Lambkin, S. Winterton,

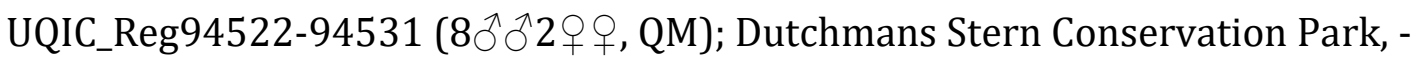
32.296944 137.983058, summit, 7.x.1997, S. Winterton, J.\&A. Skevington, C. Lambkin, UQIC_Reg94537 (1§, UQIC); Para Wirra Recreational Park, -34.701739 138.824928, 8.x.2014, A. Marquart, SLAM trap, CNC587054 (19, CNC); Sandy Creek Conservation Park, 8km NE Gawler, -34.603333 138.855833, 29.ix-4.x.1997, S. Winterton, J.\&A. Skevington, C. Lambkin, Malaise trap, malaise in heath, UQIC_Reg94542 (10ึ, UQIC); South Para Reserve, -34.676314 138.876983, 20.x.2015, A. Marquart, SLAM trap, CNC587060 (1, CNC); Tasmania: 1km SEE Gladstone, -40.580000 148.010000, 29.i.1963, I.D. Naumann \& J.C. Cardale, CNC_Diptera253802 (19, ANIC); 9 km SE Miena, -42.000000 146.766667, 1060m, 11.i.1992, G. and A. Daniels, GDCBReg_19616 (19, UQIC); Eaglehawk Neck, - 
43.019722 147.925000, 24.iv.1916, G.H. Hardy, 12, CNC_Diptera253801 (1 9 , ANIC); Erriba, -41.425350 146.137136, 30.i.1949, E.F. Riek, ANIC29034960; ANIC29034967; CNC_Diptera253710 (3웅, ANIC); Franklin-Gordon Wild River National Park, Donaghy's Hill, -42.197778 145.931944, 10-11.i.2015, J.H., A.M. and A.W. Skevington, CNC385297; CNC385305; CNC385318; CNC451889-451892; CNC451894; CNC457453 (5 ô $^{\Uparrow}, 4$ 우우, CNC); Hobart, -42.882139 147.327194, 16.xii.1917, G.H. Hardy, 45, CNC_Diptera253800 (19, ANIC); Lyell Highway, Frankin R nature trail, -42.215700 146.019800, 11.i.2015, S.J. Grove, hand collected, TMAG-

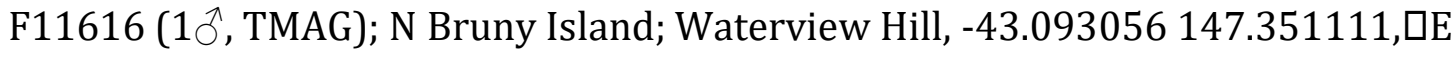
Hilltop, 31.xii.1998, J. \& A. Skevington, M. Mathieson, UQIC_Reg94797 (1へ̂, UQIC); North of Corinna, -41.588514 145.189764, 12.i.2015, J.H., A.M. and A.W. Skevington, CNC457433 (1우, CNC); Oonah, -41.224222 145.618608, 30.i.1949, E.F. Riek, ANIC29034971 (1 + , ANIC); Pump house Bay Camping Ground Arthurs Lake, 41.983333 146.866667, 12.ii.1992, D.S. Horning, Jr., in flight, CNC_Diptera253698 (1우, CNC); Samford, 1959, C.F. Ashby, Date is ambiguous reads "6.12.59", ANIC29034972 (1우, ANIC); Warra Long Term Ecological Research Site 147, -43.1 146.683333, 17.xi-16.xii.1998, malaise trap, UQIC_Reg94607-94608 (2우, UQIC); Victoria: Ararat, -37.284372 142.932553, H.W. Davey, CNC_Diptera253811 (1우, ANIC); Grampians National Park, Reed's Lookout, -37.148111 142.448261,

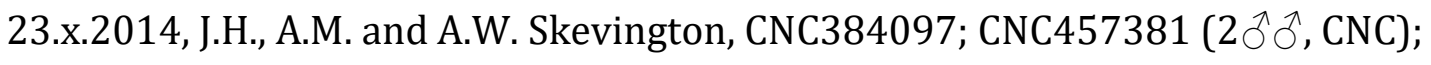
Heathmont, -37.829989 145.242019, CNC_Diptera253778 (1우,CNC); Mt. Buffalo N.P., Dicksons Falls, -36.763417 146.788092, 1440m, 29.i.2006, Jeff_Skevington_Specimen43805-43806 (2ððえ 
Buang track, -37.709467 145.676642, 4.ii.2006, M.P. van Zuijen, Jeff_Skevington_Specimen43808 (1우, UQIC); Western Australia: 2 km North of Pemberton, -34.426158 116.039058, 29.ix.1987, D.K. McAlpine, AustralianMuseumK407981 (19, AM); Geraldton, -28.777372 114.614972, ix.1926, E.W. Ferguson, CNC_Diptera253812 (19, ANIC); Barrington, Allyn Range, 41.356572 146.279756, Leptospermum, ii.1925, Label unclear: "Tps Feb '25 SU ZooExp", CNC_Diptera253810 (19, ANIC); Mt. Wellington, -42.890000 147.230000, 2.xii.2016, G.H. Hardy, Date ambiguous: "2.12.16 /48", CNC_Diptera253798 (1ð”, ANIC).

\section{Psilota darwini Young sp. nov.}

(Species plate: Fig. 19, Genitalia: Fig. 39A+B, Map: 14)

Body length: 7.5-10.1 mm.

Diagnosis: Metallic blue-green species with frons pale pilose, pleuron shiny and white pilose. Hind femur with a distinct rounded apicoventral setulose carina. Hind tibia simple. Calypter entirely pale yellow/white. Tergites pale pilose. The pale pilose pleuron and pale calypter help distinguish $P$. darwini from related species such as P. aislinni, P. alexanderi, P. coerulea, P. pollinosa, and $P$. viridis.

Description: very similar to Psilota coerulea, differing as follows: 
Thorax: Thorax metallic blue-green. Legs mostly dark brown, with apices of femora, and entire fore and mid tibia orange. Tarsomeres ranging from dark brown to orange. Hind femur with a distinct rounded apicoventral setulose carina. Hind tibia simple, with no basoventral spur. Pleuron shining, pollinosity free. Pile on pleuron white, pile on scutum and scutellum mixed black and yellow. Wing colourless, with cells c and bm bare apically. Alula completely microtrichose. Halter pale yellow. Calypter pale yellow/white.

Abdomen: Abdomen metallic blue/green. Tergites entirely white pilose. Sternites shining, white pilose.

Genitalia: Epandrium compact, about as long as tall. Cercus approximately triangular. Outer lobe of surstylus inserted just above mid-height of inner lobe, curved downwards sharply at $1 / 4^{\text {th }}$ its length. Inner lobe of surstylus very broad, approximately 3.5 times as long as wide, with a slight ventral excavation at midlength, broadening slightly towards the smoothly rounded apex. Phallapodeme smooth. Gonostylus thick, slightly darkened.

Discussion: Nothing is known about the biology of this North Queensland species. 
Etymology: Psilota darwini is named for Charles Darwin, the father of evolution.

Specimens Examined: HOLOTYPE Material: AUSTRALIA: Queensland: Dunk Island; -17.944558 146.153289; 25.VIII.1927; UQIC_Reg94804, (1へ, UQIC). Paratypes: AUSTRALIA. Northern Territory: Darwin, -12.463442 130.845642, G.F. Hill, I.B.E (144), ANIC29034807 (1へ̂, ANIC); Entire Ck 155km NE by E of Alice Springs, -23.184569 135.232533, 13.x.1978, D.H. Colless, light trap, ANIC29034463 (1, , ANIC); Queensland: same data as holotype, UQIC_Reg94691 (1q, QM); Grassy Hill, Cooktown, -15.475800 145.247100, 11.i.1982, G. and A. Daniels, GDCBReg_19670 (1ð̊, GDCB); Kuranda, -16.816878 145.633497, ANIC29034464 $(1$ ㅇ, ANIC).

\section{Psilota femoralis Schiner, 1868}

(Species plate: Fig. 20, Genitalia: Fig. 39C+D, Map: 15)

Psilota femoralis Schiner, 1868: 369. Type locality: Sydney, New South Wales, Australia.

Body length: $7.9-13.6 \mathrm{~mm}$. 
Diagnosis: Face black, densely grey pollinose, with white pile. Thorax black. Hind femur with a distinct rounded apicoventral setulose carina. Hind tibia with basoventral spur. Calypter with dark brown border. Halter dark brown. Abdomen black. Outer lobe of surstylus sharply hooked back towards base of surstylus at apex.

Description: Head: Head black, face densely grey pollinose. Face white pilose, frons and vertex black pilose. Scape and pedicel orange-brown, postpedicel brown. Postpedicel oval, approximately 1.5 times as long as wide. Eye densely white pilose.

Thorax: Thorax black. Legs mostly black, with extreme apices of femora and most of tibiae orange-brown. Hind femur with a distinct rounded apicoventral setulose carina. Hind tibia with a basoventral spur. Pleuron densely grey pollinose. Pile on pleuron white. Pile on scutum and scutellum mostly black, with some shorter yellow hairs on the scutum. Wing colourless, bare on anterior half of cells bm and cua. Cells c, dm, $r_{1}$ with small bare patches at base. Alula completely microtrichose. Halter dark brown. Calypter with border dark brown.

Abdomen: Abdomen black. Tergite 2 mostly white pilose, with posterior $1 / 5$ th black pilose. Tergite 3 white pilose anteriorly, black pilose posteriorly. Tergite 4 with mixed black and white pile. Sternites black, and white pilose. 
Genitalia: Epandrium compact, about as long as tall. Cercus approximately triangular, slightly elongate. Outer lobe of surstylus inserted near top of inner lobe, curved downwards slightly on apical $1 / 3^{\text {rd }}$, with apex of lobe thickened and hooked sharply downwards and backwards towards base of surstylus at a $90^{\circ}$ angle. Inner lobe of surstylus broadening over entire length, with a slight ventral excavation at $1 / 3^{\text {rd }}$ its total length, rounded at apex. Ejaculatory apodeme with small ventral projection. Gonostylus not darkened.

Discussion: This species has been collected on Leptospermum J.R. Forst. and G. Forst (Myrtaceae) blossoms.

Specimens Examined: AUSTRALIA. New South Wales: 24 km W of South Grafton, -29.687439 152.692319, 3.x.1985, R. Eastwood, GDCBReg_19564; GDCBReg_19566 $(2 \widehat{\partial}, \mathrm{GDCB}) ; 5 \mathrm{~km}$ East of Bilpin, Blue Mountains, -33.462750 150.572836, 22.ix.1977, N.W. Rodd, AustralianMuseumK410292 (19, AM); Blue Mtn., 40km ENE Glen Innes, -29.641186 152.109278, 15.xii.1984, D.K. Yeates, UQIC_Reg9457094571 (2ððૈ, UQIC); Broadwater N. N.S.W., -29.050325 153.427539, 22.viii.1981, D. Yeates, UQIC_Reg94584 (1§^, UQIC); Broadwater Nat. Pk., -29.050325 153.427539, 6.ix.1981; 26.viii.1984, G. Daniels; D.K. Yeates, GDCBReg_19654; UQIC_Reg9458094581; UQIC_Reg94583; UQIC_Reg94587 (5 §̂ึ Qึ QM, GDCB); Broadwater, -

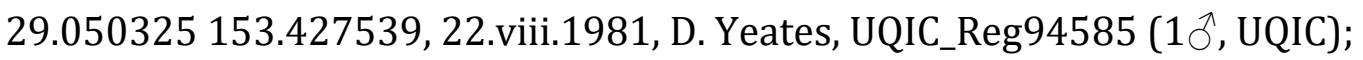


French's Forest, -33.749000 151.233100, 9.ii.1936, D.F. Waterhouse, ANIC29034913 (1へ, ANIC); Gibraltar Ra. N.P., Waratah track, -29.548814 152.286936, on Leptospermum sp. blossom, 10-12.xi.1984, D.K. Yeates, UQIC_Reg94566-94567 (2へðへ, UQIC); Goonderra Ridge, Royal National Park, 34.114342 151.046586, 29.i.1979; 2.x.1978; 21.iii.1979; 22.viii.1976; 24.x.1976; 28.ix.1975; 4.x.1978; 6.x.1975; 2.x.1978; 28.ix.1975; 6.x.1975, G. and A. Daniels, GDCBReg_19682; GDCBReg_19672-19674; GDCBReg_19701-19702;

GDCBReg_19706; GDCBReg_19708-19711; GDCBReg_19750-19758; GDCBReg_19761; GDCBReg_19747-19748; GDCBReg_19648-19652 (21ððð,7우우, GDCB); Ku-ring-gai Chase National Park near Sydney, -33.637567 151.233900,

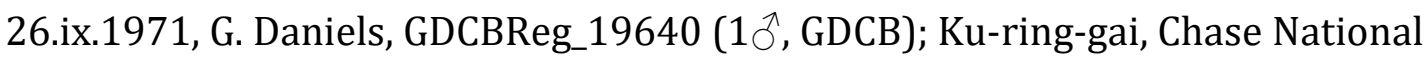
Park, -33.637567 151.233900, 2.x.1972, G. and A. Daniels, GDCBReg_19727 (1ㅇ, GDCB); Lawson, Blue Mtns., -33.716667 150.433333, 23.x.1976, G. Daniels, G. Daniels Collection, GDCBReg_19765 (1ðð, GDCB); Mount Boyce, Blue Mts., 33.615789 150.266597, 30.xi.1975, G. Daniels, G. Daniels Collection, GDCBReg_19723 (10ึ, GDCB); Mt. Kuring-Gai, -33.645433 151.131150, 27.ix.1950, B. McMillan, UQIC_Reg94666 (1へð, UQIC); Mt. Westmacott nr. Waterfall, -34.138853 150.980736, 24.iii.1979, G. Daniels, GDCBReg_19722 (1ðð, GDCB); Mt. Westmacott, nr Waterfall, -34.138853 150.980736, 24.iii.1979, G. Daniels, GDCBReg_1965719659 (3ðึ ڤึ, GDCB); Royal National Park, -34.114342 151.046586, 29.viii.1976; 6.x.1975, G. and A. Daniels, GDCBReg_19745-19746 (2ðð, GDCB); Stanwell Tops, 34.219903 150.991808, 5.ii.1978; 9.x.1977, G. Daniels, GDCBReg_19742-19743;

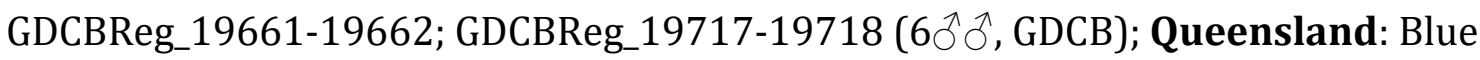


Mtn., 4km NE Stanthorpe, SE Qld., -28.631058 151.985794, 977m, summit, 28.ix.1987, D.K. Yeates, UQIC_Reg94569 (1つ̂, UQIC); Brisbane, Mount Coot-tha, 27.487778 152.950558, 170m, hilltop, 7.iii.1998; 13.iv.1997, J. \& A. Skevington, UQIC_Reg94602; UQIC_Reg94551 (2ððへ, UQIC); Brisbane, -27.526975 153.040322, 11.x.1958; 7.ix.1927, M. Permiakoff; J. Mann, UQIC_Reg94708; UQIC_Reg94823 ( $2 \AA \widehat{\jmath}$, UQIC); Brown Lake, N. Stradbroke Is., S.E.Q., -27.490108 153.432378, on Leptospermum flavescens blossom, 21-24.ix.1984, D.K. Yeates \& R. de Keyzer; D.K. Yeates, UQIC_Reg94575-94578 (4仓̊̋, UQIC); Moreton Island, Mount Tempest, 27.149989 153.399994, 285m, hilltopping, 20.ix.1997, J. \& A. Skevington, UQIC_Reg94727-94729 (3ððð, UQIC); Mount Moffatt, Mt. Moffatt sect., Carnarvon Nat. Pk., -25.165167 147.840419, 26.ix.1986, D.K. Yeates, UQIC_Reg94572-94573 (1へ̂, UQIC); Mount Tinbeerwah, nr Cooroy, -29.950000 152.433333, 29.ix.1985; 6.ix.1980; 24.viii.1980; 6.ix.1980, G. and A. Daniels, GDCBReg_19555-19556; GDCBReg_19558; GDCBReg_19665; GDCBReg_19675-19676; GDCBReg_19677

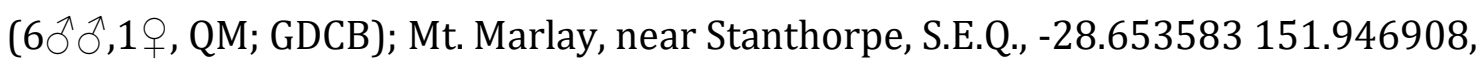
6-9.xi.1984, D.K. Yeates; Noosa, -26.364544 152.967669, 27.viii.1959, F.A. Perkins, UQIC_Reg94718 (10ึ, UQIC); Tin Can B., -25.919297 153.003278, Leptospermum + shrubs, 21.viii.1957, F.A. Perkins, sweeping, UQIC_Reg94719 (19, UQIC); Wild Horse Mountain near Beerburrum, -26.916667 152.150000, 28.ix.1985; 16-17.ix.1985, G. and A. Daniels; R. Eastwood, GDCBReg_19547; GDCBReg_19553-19554 (3ðð GDCB); Sunnybank, 18.ix.1927, UQIC_Reg94683 (10̂, UQIC). 


\section{Psilota livida Young and van Steenis sp. nov.}

(Species plate: Fig. 21, Genitalia: Fig. 39E+F, Map: 16)

Body length: 7.3-8.2 mm.

Diagnosis: Metallic blue species with face thinly white pollinose. Frons with mixed black and white pilosity, vertex dark pilose. Pleuron white pilose, scutum white pilose anterior to transverse suture, mostly black pilose behind transverse suture. Scutellum mostly black pilose. Hind leg simple. Halter dark brown. Calypter pale.

Description: Head: Head black, face shining blueish-black, with thin white pollinosity covering the sides of the face. Central stripe of face shining, free of pollinosity. Face white pilose, frons with mixed black and white pilosity, vertex black pilose. Antenna entirely orange, baseoflagellomere oval, approximately 1.5 times as long as wide. Eye densely white pilose.

Thorax: Thorax blueish-black, shining and free of pollinosity. Legs mostly dark brown, with apices of fore- and mid femora, basal $1 / 3^{\text {rd }}$ of fore tibia orange. Hind leg simple. Pleuron shining, pollinosity free. Pile on pleuron white, pile on scutum white anterior to transverse suture, mostly black posterior to suture. Scutellum mostly black pilose, with a few white pili. Wing colourless, with cell bm entirely bare, cell 
cua bare on anterior half, cells $c$, and $\mathrm{r}_{1}$ bare on basal half, and cells $\mathrm{m}_{4}, \mathrm{dm}$, and $\mathrm{r}_{4+5}$ bare on basal $1 / 4^{\text {th }}$. Alula completely microtrichose. Halter white. Calypter white.

\begin{abstract}
Abdomen: Abdomen blueish-black. Tergite 1 white pilose, tergite 2 mostly white pilose with a few short black pili medially, tergite 3 white pilose laterally with many black pili mediall, tergite 4 mostly white pilose with a few black pili medially. Sternites black, white pilose.
\end{abstract}

Genitalia: Epandrium compact, about as long as tall. Cercus approximately triangular. Outer lobe of surstylus inserted at mid-height and mid-length of the inner lobe, short, thick and curved smoothly downwards over its entire length. Inner lobe of surstylus long and straight, approximately 4 times as long as wide (at base), with a semicircular ventral excavation at $2 / 3^{\text {rds }}$ its length, almost square at apex. Phallapodeme smooth. Gonopods thick, not darkened.

Discussion: This species has been collected in a Eucalyptus woodland at a mecury vapour light, otherwise nothing is known about its biology

Etymology: "livida" derives from the Latin lividus, meaning "blueish black, or leaden". This is in reference to the blueish-black abdomen of Psilota livida. 
Specimens Examined: HOLOTYPE Material: 6km N Taroom; -25.600000

149.766667; eucalyptus woodland, 25.XI.1992; coll.G. Daniels; (1ð GDCB).

AUSTRALIA: Queensland: Mcllwraith Range 8km NE Coen, -13.888333

143.253500, 200m; mercury vapour light, 7.I.1995, G. and A. Daniels, GDCBReg_19609; GDCBReg_19571, (1우 GDCB).

Psilota longipilosa Young and Thompson sp. nov. (Species plate: Fig. 22, Genitalia: Fig. 39G+H, Map: 17)

Body length: 11.9-14.1 mm.

Diagnosis: Metallic blue species with face yellow pilose, frons extensively black pilose. Pleuron white pollinose and white pilose. Hind femur with a low apicoventral setulose carina. Hind tibia with a basoventral spur. Scutum with long, black pile.

Description: Head: head black, face densely pale pollinose. Face yellow pilose, frons and vertex extensively black pilose. Antenna orange, dull brown dorsally on scape, pedicel, and postpedicel. Eye densely yellow pilose. 
Thorax: Thorax metallic blue. Femora dark, with only apices orange. Fore and mid tibia orange. Fore and mid femur white pollinose. Hind femur with a low apicoventral setulose carina. Hind tibia with a basoventral spur. Pleuron white pollinose. Pile on pleuron white. Pile on scutum and scutellum long, approximately half the length of the arista, and entirely black. Metepimeron and katepimeron bare. Wing hyaline, with cell $\mathrm{c}$ bare on ventral half, cell $\mathrm{r}$ bare anteriorly and ventrally, and cells bm, Cua, and the anal lobe bare anteriorly. Alula completely microtrichose. Halter dark brown. Calypter pale brown, with a dark brown border.

Abdomen: Abdomen metallic blue. Tergites 2, 3 and 4 with white pile on basal half and black pile on apical half. Tergite pile long, approximately one-third the length of the arista. Sternites shining, white pilose.

Genitalia: Epandrium compact, about as long as tall. Cercus approximately triangular. Outer lobe of surstylus inserted just above mid-height of inner lobe, curved downwards smoothly over entire length. Inner lobe of surstylus short, barely extending past outer lobe, widest at basal ventral swelling, with many long, strong setae present ventrally at base, widening only slightly past midpoint to a rounded apex. Phallapodeme with a ventral, forward facing projection. Gonostylus thick, not darkened. 
Discussion: Nothing is known about the biology of this species.

Etymology: longi- derives from the Latin longus, meaning "long", and pilosa derives from the scientific term pile, which refers to the hair-like structures covering some tergites in Syrphidae. This is reference to the unusually long pile covering the body of Psilota longipilosa, especially on the face.

Specimens Examined: HOLOTYPE Material: 26km S of Dwellingup; -32.990108 116.055408; coll.I.D. Naumann, J.C. Cardale; ANIC29034978 (10ึ, ANIC). Paratypes: AUSTRALIA. Western Australia: same data as holotype, USNM_ENT249842 $(10$, ANIC); 5 miles East of Nornalup, -35.004314 116.880339, 8.x.1970, D.H. Colless,

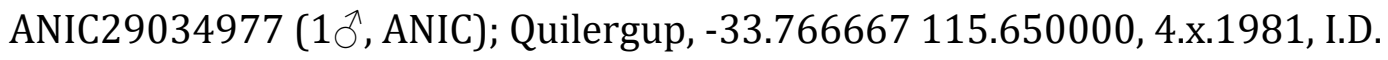
Naumann, J.C. Cardale, ANIC29034976 (1ð, ANIC).

Psilota maculata Young and Van Steenis sp. nov. (Species plate: Fig. 23, Genitalia: Fig. 40A+B, Map: 18)

Body length: 8.7-11.5 mm.

Diagnosis: Pleuron shining, free of pollinosity. Scutum with mixed black and white pile. Hind leg simple. Tergites 3 and 4 yellow, with large black 
triangular markings medially. Tergite 4 mostly or entirely black, usually with anterolateral yellow markings.

Description: Head: Head black, face sparsely pale pollinose. Face pale yellow pilose with some black pili mixed in on upper half of face, frons mostly black pilose with a few pale pili, vertex black pilose. Scape and pedicel orange, postpedicel dark brown with only ventral edge orange, oval, approximately 1.5 times as long as wide. Eye densely brown pilose.

Thorax: Thorax black. Fore- and mid leg orange, hind leg dark brown. Hind femur unmodified, with only a few weak, black setulae apicoventrally. Pleuron shining, free of pollinosity. Pile on thorax mixed black and yellow. Wing colourless, with apical $1 / 2$ of cell bm, apical $3 / 4^{\text {ths }}$ of cell cua, and basal $1 / 3^{\text {rd }}$ of cell c bare. Alula microtrichose. Halter pale yellow. Calypter light brown with a dark brown border.

\footnotetext{
Abdomen: Tergite 1 black. Tergites 2 and 3 yellow with a large, triangular median black mark covering most of anterior edge of each tergite, narrowly meeting posterior edge. Tergite 4 entirely black or with lateral edges yellow on anterior half. Tergites shining, free of pollinosity. Tergites 1 and 2 with some pale pilosity laterally, otherwise tergites black pilose. Sternites yellow, pale pilose.
} 
Genitalia: Epandrium compact, about as long as tall. Cercus approximately triangular. Outer lobe of surstylus inserted at top of inner lobe, thin, and abruptly curved downwards at about $2 / 3^{\text {rds }}$ its length. Inner lobe of surstylus long, thin, and straight, approximately 5 times as long as wide (at base), slightly increasing in width over entire length, with apex square. Phallapodeme smooth. Gonopods slightly constrictd medially, slightly darkened.

Discussion: Nothing is known about the biology of this species.

Etymology: macula is Latin for "spot, speckle, or variegation". This is in reference to the triangular spots on the abdominal tergites of Psilota maculata.

Specimens Examined: HOLOTYPE Material: AUSTRALIA: Queensland: Mount Moffatt, Mt. Moffatt sect., Carnarvon Nat Pk; -25.058100 148.044400; 26.IX.1986; D.K. Yeates; UQIC_Reg94640 (1 $\overbrace{}^{\Uparrow}$ UQIC). Paratypes: AUSTRALIA. New South Wales: Stanwell Tops, -34.219903 150.991808, 5.ii.1978, G. Daniels, GDCBReg_19604 (1へ̂, GDCB); Queensland: Amiens nr. Stanthorpe, -28.600522 151.799608, 25.xi.1979, G. Daniels, GDCBReg_19689 (1へ̂, GDCB); Carnarvon National Park, Mount Moffatt Summit, -25.058100 148.044400, 1100m, woodland, 5.xi.2010, C. Lambkin et al.,

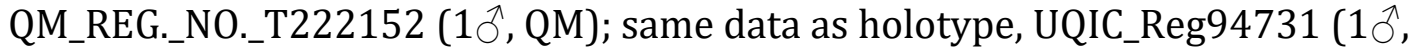


UQIC); Wild Horse Mountain near Beerburrum, -26.916667 152.150000, 28.ix.1985,

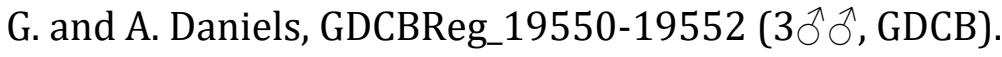

Psilota mcqueeni Young sp. nov.

(Species plate: Fig. 24, Genitalia: Fig. 40C+D, Map: 19)

Body length: $7.9 \mathrm{~mm}$.

Diagnosis: Metallic blue-black species with face free of pollinosity except around eye margin. Frons pale pilose, vertex dark pilose. Thorax pale pilose, with some dark pili on scutum. Hind femur with a distinct triangular apicoventral setulose carina. Hind tibia simple. Halter white. Calypter pale. Tergite 4 white pilose.

Description: Head: Head black, face shining blueish-black, with pollinosity only around eye margin. Face and frons white pilose, vertex black pilose. Antenna dark brown, baseoflagellomere oval, approximately twice as long as wide. Eye densely white pilose.

Thorax: Thorax blueish-black, shining and free of pollinosity. Legs mostly dark brown, with apices of fore- and mid femora, basal $1 / 3^{\text {rd }}$ of fore and mid tibia orange. 
Hind femur with a distinct triangular apicoventral setulose carina. Hind tibia simple, with no basoventral spur. Pleuron shining, pollinosity free. Pile on pleuron and scutellum entirely white, pile on scutum mostly white with some dark pili. Wing colourless, with cells $\mathrm{c}, \mathrm{r} 1$, and cua bare on basal half, cells bm, and br mostly bare. Alula completely microtrichose. Halter white. Calypter white.

Abdomen: Abdomen blueish-black. Tergites white pilose laterally, with short black pile medially. Sternites black, white pilose.

Genitalia: Epandrium conpact, about as long as tall. Cercus approximately triangular, with apex slightly concave. Outer lobe of surstylus inserted at top of inner lobe, thin, and curved smoothly downwards over its entire length, also curving inwards so the apex loops under the inner lobe of the surstylus. Inner lobe of surstylus long and straight, approximately 4 times as long as wide (at base), with a semicircular ventral excavation at $2 / 3^{\text {rds }}$ its length, almost square at apex. Inner lobe of surstylus also with a large medial projection on the dorsal half of the inner surface at $1 / 2$ its length. Phallapodeme smooth. Gonopods slightly darkened.

Discussion: Nothing is known about the biology of Psilota mcqueeni. 
Etymology: This species is named for Peter McQueen, my maternal grandfather who died while I was visiting collections for this project in Australia. Granddad was always supportive of my interest in natural history, and was always a positive influence in my life.

\section{Specimen Examined: HOLOTYPE Material: AUSTRALIA: Northern Territory:}

Koongarra, 15 km E of Mt. Cahill, -12.865225 132.843317, 6-9.III.1973, coll.D.H. Colless, USNM_ENT_00249848 (1○^ USNM).

\section{Psilota metallica Young and Thompson sp. nov.}

(Species plate: Fig. 25, Genitalia: Fig. 40E+F, Map: 20)

Body length: $10.4-12.3 \mathrm{~mm}$.

Diagnosis: Strongly iridescent over entire body, blue/green thorax and red/green abdomen. Hind leg simple.

Description: Head: Frons and face shining metallic blue and green, bare of pollinosity. Face, frons, and vertex with long white pile. Antenna orange. Eye densely pilose, pile entirely white. 
Thorax: Thorax entirely iridescent blue andgreen, shining, pollinosity free. Legs dark blue, orange at extreme apex of femora and base of tibiae. Hind femur unmodified, with only a few weak, black setulae apicoventrally. Pleuron white pilose. Scutum mostly white pilose, with some black pile laterally ventrad of transverse suture. Scutellum white pilose, with distinct marginal sulcus. Wing colourless, entirely bare.

Abdomen: Tergites entirely iridescent green and red. Tergites 2-3 with some short dark pile on disc, all tergites white pilose laterally. Tergite 4 entirely white pilose. All sternites iridescent blue and red.

Genitalia: Epandrium compact, about as long as tall. Cercus approximately triangular. Outer lobe of surstylus inserted at about mid-height of inner lobe, abruptly curved downwards at base. Inner lobe of surstylus broad, approximately 3 times as long as wide, swollen at base, slightly constricted, and smoothly rounded at apex. Phallapodeme smooth, gonopods not darkened.

Discussion: This species has been collected from flowers of Hakea leucoptera R. Br. (Proteaceae). Otherwise nothing is known of its biology. 
Etymology: metallica derives from the Latin metallicum, meaning "metal". This is in reference to the strongly iridescent cuticle of Psilota metallica.

\section{Specimens Examined: HOLOTYPE Material: AUSTRALIA: New South Wales; 99}

km North of Wentworth; -33.027339 150.952214; Hakea leucoptera; 30.XI.1992; coll.N.W. Rodd; AustralianMuseumK410178; (1 $\overbrace{}^{\Uparrow}$ AM). Paratypes: AUSTRALIA. New

South Wales: same data as holotype, AustralianMuseumK410160 (1 $\left.\partial^{\lambda}, \mathrm{AM}\right)$;

Pooncarie, -33.383350 142.566942, 30.xi.1993, N.W. Rodd, AustralianMuseumK410169 (1ㅇ, AM); Tibbooburra, Cobham Lake, -29.426133 142.001994, 17.x.1949, S.J.K. Paramonov, USNM_ENT_00249839 (1ㅇ, USNM); Queensland: Durham Downs, Nokandra, -26.116933 149.076992, 13.xi.1949, S.J. Paramonov, "leg", USNM_ENT_00249840 (1ðへ, USNM); Talawanta, 80 ml. S.E. of Burketown, -29.601372 147.060083, 24.v.1972, G.B. \& S.R. Monteith, UQIC_Reg94706 (1つ̋, UQIC).USNM_ENT_00249839; USNM_ENT_00249840; Jeff_Skevington_Specimen26984; UQIC_Reg94706, $\left(3 \partial^{\jmath}, 2\right.$, 2 우,1 ex AM; USNM; CNC; UQIC).

\section{Psilota nigripilosa Young sp. nov.}

(Species plate: Fig. 26, Genitalia: Fig. 40G+H, Map: 21)

Body length: $11.9 \mathrm{~mm}$. 
Diagnosis: Face black, densely white pollinose, with black pile. Frons and vertex black pilose. Thorax black. Hind femur with a low triangular apicoventral setulose carina. Hind tibia with basoventral spur. Calypter with dark brown border. Halter pale. Abdomen black. Lateral edges of tergite 3 black pilose. Male genitalia with inner lobe broadest near apex. The black pile on the face of $P$. nigripilosa distinguishes it from the similar P. auripilosa, while the inner lobe of the surstylus broadest near apex distinguishes it from P. spathistyla.

Description: very similar to Psilota spathistyla, differing as follows:

Abdomen: Tergite 3 entirely black pilose.

Genitalia: Epandrium compact, about as long as tall. Outer lobe of surstylus inserted at bottom of inner lobe, bent smoothly downwards at midlength, and then swollen and bent slightly backwards at apex. Inner lobe of surstylus broadening over entire length, with a large ventral excavation at midlength, rounded at apex. Phallapodeme smooth. Gonostylus thick, not darkened. 
Discussion: The lone specimen of this species was collected at a rainforest tree trunk flux. As it is a male, it was not ovipositing at this location, but it may have been looking for a female that would be, or simply feeding on the decaying tree sap.

Etymology: nigri- derives from the Latin niger, which means "black" or "dark", and pilosa derives from the scientific term pile, which refers to the hair-like structures covering some tergites in Syrphidae. This is in reference to the dark pile on the face of Psilota nigripilosa that distinguishes it and Psilota spathistyla from Psilota auripilosa.

\section{Specimens Examined: HOLOTYPE Material: AUSTRALIA: Queensland:}

Charmillin Creek, -17.700000 145.516667, 940mm, rainforest, treetrunk flux, 1.XII.1997, coll.G. Monteith, QM_REG._NO._T223229, (10へ QM).

\section{Psilota nitida Macquart, 1850}

(Species plate: Fig. 27, Genitalia: Fig. 41A+B, Map: 22)

Psilota nitida Macquart, 1850: 449. Type locality: Tasmania, Australia.

Body length: $7.1-12.2 \mathrm{~mm}$. 
Diagnosis: Face black, densely white pollinose, with white pile. Frons and vertex black pilose. Thorax black. Hind femur with a distinct rounded apicoventral setulose carina. Hind tibia with basoventral spur. Calypter with dark brown border. Halter pale, sometimes with knob slightly pigmented. Abdomen black. Anterior half of tergites 2 and 3 pale pilose.

Description: very similar to Psilota spathistyla, differing as follows:

Head: Head black, face pale pollinose. Face pale pilose.

Thorax: Legs variable, ranging from mostly orange to almost entirely dark brown, with only apices of femora and baes of tibiae orange. Hind femur with a distinct rounded setulose carina. Pile on scutum mixed black and yellow anterior to transverse suture, usually entirely black posterior to suture. Scutellum usually entirely black pilose, with only scutellar fringe pale pilose. Wing colourless.

Abdomen: Abdomen black. Tergites 2 and 3 white pilose on anterior $2 / 3^{\text {rds }}$, and black pilose on posterior $1 / 3^{\text {rd }}$. Tergite 4 with mixed black and white pile. 
Genitalia: Epandrium compact, about as long as tall. Cercus approximately triangular. Outer lobe of surstylus inserted just above mid-height on inner lobe, curved downwards on apical $1 / 3^{\text {rd }}$, with apex of lobe thickened and curved slightly backwards towards base of surstylus. Inner lobe of surstylus broadening over entire length, with a slight ventral excavation at midlength, rounded at apex. Phallapodeme smooth. Gonostylus thick, not darkened.

Female: Similar to male aside from sex-specific differences. Pile on thorax much shorter than male, pile of scutum entirely yellow to entirely black.

Discussion: Psilota nitida exhibits more variation in pleural pile and leg colouration than most Psilota species, and may represent a complex. However, no definite characters could be found to reliably separate colour morphs, and so it is here treated as a single species.

Specimens Examined: AUSTRALIA. Australian Capital Territory: Canberra, 35.282000 149.128686, 1-10.xii.1953, F.M. Hull, CNC_Diptera254993 (1へ̂, CNC); 14.xii.1959, Z. Liepa, ANIC29034894 (1 + , ANIC); 18.xi.1934, M. Fuller, ANIC29034895 (1ㅇ, ANIC); 8.ii.1948, S.J. Paramonov, ANIC29034994 (1へ, ANIC); Cotter River, -35.531650 148.875125, 29.xi.1962, Z. Liepa, ANIC29034886 (1우, ANIC); Paddy's River, Canberra, -35.282000 149.128686, 18.i.1959, K.R. Norris, 
ANIC29034887 (1ㅇ, ANIC); New South Wales: 10 km West of Murwillumbah, 28.331308 153.281278, 25-27.ix.1981, N.W. Rodd, AustralianMuseumK407028; AustralianMuseumK410613 (2우, AM); 3 km North of Lansdowne, via Taree, 31.758753 152.534258, on Acradenia euodiiformis, 19.ix.1991, G. Williams, AustralianMuseumK410248 (1ð̄, AM); Armidale, -30.501561 151.666203, 24.i.1960; 29.i.1960, C.W. Frazier, CNC_Diptera253691-253692; ANIC29034988

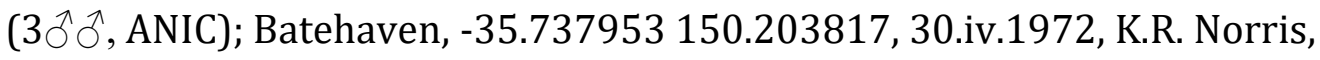
ANIC29034889 (1우, ANIC); Bateman's Bay 4 m. N., -35.658458 150.177744, 20.x.1953, G. Daniels, CNC_Diptera255023 (1へへ, CNC); Bellmore Falls, -34.633958 150.555914, 1948, S.J. Paramonov, ANIC29034884 (1우, ANIC); Concord, near Sydney, -33.926375 151.125936, 7.xii.1968, R. Coveney, AustralianMuseumK410277 (1ㅇ, AM); East of Taree Manning Point, -31.894433 152.658919, littoral rainforest on Acmena smithii blossoms, 3.xi.1990, G. Williams, AustralianMuseumK408013 (1ð^, AM); Gundamain, National Park, 23.viii.1925, Mackerras, ANIC29034991 (1ð̊, ANIC); Mooney Mooney CK nr Gosford, -33.437894 151.246467, 1.i.1977, G. Daniels, GDCBReg_19771 (1 $\overbrace{}^{\lambda}$, GDCB); Mount Tomah, Blue Mountains, -33.549614 150.416033, 16.ii.1986, N.W. Rodd, AustralianMuseumK410179 (1q, AM); Nullo Mountain 20 miles Northeast of Rylstone, -32.737947 150.226400, 12.xi.1950, T.G. Campbell, ANIC29034979 (1ð, ANIC); Nullo Mountains, 20 miles Northeast of Rylstone, -32.737947 150.226400, 12.xi.1950, T.G. Campbell, ANIC29034885 (1, ANIC); Orange, -33.284228 149.102683, 25.xii.1951, Z. Liepa, ANIC29034888 (1ㅇ, ANIC); Tregeagle, 10 km SE Lismore, -26.233333 153., 25.vii.1984, D.K. Yeates, UQIC_Reg94589 (1つ̋, UQIC); 
Winburndale National Reserve, Northeast of Bathurst, -33.333164 149.789450, 6.xii.1974, J. Cardale, ANIC29034882 (1우, ANIC); [Royal] National Park, -34.076208 151.054569, 1.i.1930, L.M. Willings, ANIC29034883 (1우, ANIC); near Camden Head , -31.644103 152.829400, littoral rainforest, 4.i-5.ii.2000, G. \& T. Williams, Malaise trap, SEPP 26 Site 122, AustralianMuseumK410286 (1, AM); Queensland: 2 km W of Rainbow Beach, -25.966667 153.150000, heathland; rainforest, 24.ix.1994, G. \& A. Daniels \& C.J. Burwell, GDCBReg_13080; GDCBReg_19577; GDCBReg_19580; GDCBReg_19585-19587 (2ð^,4ㅇ, GDCB); Bluff Ra. foothills, Biggenden, -25.512597 152.037758, ix.1974, H. Frauca, ANIC29034485 (1ㅇ, ANIC); Brisbane, -27.526975 153.040322, 10.ix.1927; 7.ix.1927; 16.ix.1962; 3.ix.1955, J. Mann; C.F. Ashby; F.A. Perkins, UQIC_Reg94812; ANIC29034987; ANIC29034989; UQIC_Reg94596; UQIC_Reg94817; UQIC_Reg94701 (5つ̋ð,1ำ, QM; ANIC); Cairns District, -16.918678

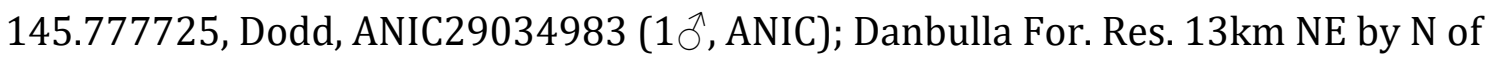
Yungaburra, -17.271111 145.583058, 17.xi.1981, D.H. Colless, Malaise trap, ANIC29034487 (1, ANIC); Dunk Island, -17.944558 146.153289, 25.viii.1927, ANIC29034982; UQIC_Reg94684; UQIC_Reg94685-94690; UQIC_Reg94805 (9ðð ANIC; QM); Enoggera Res., site 3, -27.450000 152.916667, 100m, 27.i-15.iii.2000, Burwell, Evans, Malaise trap, QM Samp. \#50274, QM_REG._NO._T222143-222144 (2甲, QM); Iron Range, Cape York Pen., N. Qld., -12.700008 143.300000, 1623.xi.1965, G. Monteith, UQIC_Reg94663 (19, UQIC); Jamboree Heights, Brisbane, 27.552778 152.930556, 20m, flying about compost bin, 7.x.2001, G. Daniels, GDCBReg_17094 (19, GDCB); Lamington NP, -28.188000 153.121000, 2-19.ii.2009, F. Turco, Malaise trap, QM_REG._NO._T222164 (1우, QM); Landsborough, 2.55km NE, 
$-26.789000152 .978000,55 \mathrm{~m}$, rainforest, 24.ix-9.x.2010, G. Monteith, Malaise trap, QM_REG._NO._T222160 (1ㅇ, QM); Landsborough, 2.66km NE, -26.790000 152.980000, wet sclero, 9-24.i.2011, G. Monteith, Malaise trap, QM_REG._NO._T222159 (19, QM); Mt Coolum, -26.900000 153.083333, rainforest, 17.ix.1989, R. Eastwood, GDCBReg_19517 (1우, GDCB); Mt Marlay near Stanthorpe , 28.666667 151.933333, summit, 11.x.1998, J. Skevington, P. Bouchard, C. Lambkin,

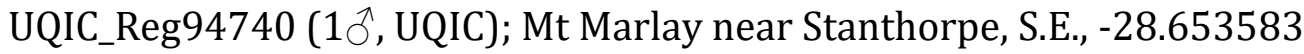
151.946906, on Leptospermum brevipes, 30.x.1985, D.K. Yeates, UQIC_Reg94662 (1仓ે, UQIC); North West Is., near Yeppoon, -23.295058 151.707692, 17-22.viii.1968, D.M. Reeves, UQIC_Reg94601 (1§, UQIC); Palm Island, -18.689428 146.539225, 20.xii.1930-6.i.1931, I.M. Mackerras, ANIC29034486 (1, ANIC); Bancroft, ANIC29034984 (1ðへ, ANIC); Rockpool Gorge Mt Walsh Nat Park Biggenden, -

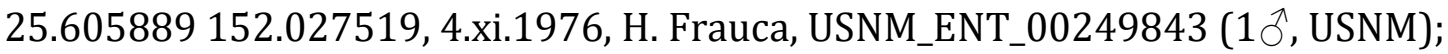
Rockpool Gorge, Mount Walsh National Park, Biggenden, -25.605889 152.027519, 4.x.1976, H. Frauca, ANIC29034990 (1ð , ANIC); Tallegalla Weir Junction, 25.757000 152.695000, 35m, 6-22.xii.2011, G. Monteith, Malaise trap, QM_REG._NO._T222162 (1ㅇ, QM); 7-22.xi.2011, G. Monteith, Malaise trap, QM_REG._NO._T222156 (19, QM); The Blunder,-27.526975 153.040322, 18.ix.1966; 26.ix.1966; 15.x.1967, C.F. Ashby, CNC_Diptera253690; ANIC29034488; ANIC29034985 (3ðึ ठึ,1ㅇ, ANIC); Toowong, -27.483333 152.983333, 3.iii.2000, UQIC_Reg94721 (1어,UQIC); Yarraman, 2.2km SSE, -26.857000 151.994000, 12.iii10.iv.2010, G. Monteith, Malaise trap, QM_REG._NO._T222157-222158; QM_REG._NO._T222161 (3웅, QM); Yeppoon, -23.133408 150.733383, 9.ix.1924, 
ANIC29034452 (1우, ANIC).

Psilota pallidifacies Young and van Steenis sp. nov. (Species plate: Fig. 28, Genitalia: Fig. 41C+D, Map: 23)

Body length: 7.7-8.0 mm.

Diagnosis: Face orange, densely white pollinose, with white pile. Thorax metallic blue. Hind femur with a distinct rounded apicoventral setulose carina. Hind tibia simple. Calypter pale. Halter pale. Abdomen metallic green. The orange face of $P$. pallidifacies distinguishes it from the similar $P$. viridis.

Description: Head: Head dull orange, face densely white pollinose. Face and frons white pilose, vertex black pilose. Scape and pedicel entirely orange, postpedicel brown on apical edge and orange below. Postpedicel oval, approximately 1.5 times as long as wide. Eye densely white pilose.

Thorax: Thorax metallic blue. Legs almost entirely orange, with femora orangebrown. Hind femur with a distinct rounded apicoventral setulose carina. Hind tibia simple, with no basoventral spur. Pleuron shining, free of pollinosity. Pile on pleuron white. Pile on scutum and scutellum mixed black and white. Wing 
colourless, extensively bare on basal half. Cells c, br, bm, $\mathrm{r}_{1}$ entirely bare. Cells cua, $\mathrm{m}_{4}, \mathrm{dm}, \mathrm{r}_{4+5}$, and $\mathrm{r}_{2+3}$ bare on basal half. Alula completely microtrichose. Halter pale. Calypter pale.

Abdomen: Abdomen metallic green. Abdomen entirely white pilose. Sternites metallic green, and white pilose.

Genitalia: Epandrium compact, about as long as tall. Cercus approximately triangular. Outer lobe of surstylus inserted at mid-height of inner lobe, curved downwards on apical $1 / 3^{\text {rd }}$, with apex of lobe thickened and curved backwards towards base of surstylus. Inner lobe of surstylus broadening over entire length, with a large ventral excavation at midlength, rounded at apex. Phallapodeme smooth. Gonostylus slightly darkened.

Discussion: Nothing is known about the biology of this species.

Etymology: pallid- derives from the Latin pallidus, meaning "pale" or "pallid", and facies is Latin for "face, countenance, or visage". This is in reference to the relatively pale orange face of Psilota pallidifacies, which distinguishes it from Psilota viridis. 
Specimens Examined: HOLOTYPE Material: Sunnybank, -27.578997 153.059008, 18.IX.1927, UQIC_Reg94550, (1○̂ UQIC). AUSTRALIA: Queensland: AUSTRALIA: Queensland: Dunwich, North Stradbroke Island; -27.500000 153.400000; 22.VIII.1958; coll.S.S. Sekhon; UQIC_Reg94720; (1ㅇ UQIC).

Psilota pollinosa Young and van Steenis sp. nov. (Species plate: Fig. 29, Genitalia: Fig. 41E+F, Map: 24)

Body length: $9.8-13.2 \mathrm{~mm}$.

Diagnosis: Metallic blue species with pleuron white pollinose and white pilose. Hind femur with a distinct rounded apicoventral setulose carina. Hind tibia simple. The pollinose pleuron distinguishes $P$. pollinosa from the similar $P$. coerulea, P. aislinni, and P. alexanderi.

Description: very similar to Psilota coerulea, differing as follows:

Thorax: Legs somewhat more orange in most specimens. Apical half of fore- and mid femora often with an anterior orange streak, fore- and mid tibia partly to entirely orange-brown. Pleuron white pollinose. Pile on pleuron almost entirely 
white, with some individual black pili on the posterior anepisternum and anepimeron. Halter orange-brown.

Abdomen: Tergites 2 and 3 almost entirely white pilose, with only posterior margin black pilose.

Genitalia: Epandrium compact, about as long as tall. Cercus approximately triangular. Inner lobe of surstylus inserted near top of inner lobe, bent sharply downwards at $2 / 3^{\text {rds }}$ its length, slightly swollen at apex. Inner lobe elongate, with a ventral bulge near base, excavated ventrally at $1 / 3^{\text {rd }}$ its length and then widening smoothly to apex. Phallapodeme smooth. Gonostylus thick, not darkened.

Discussion: This species has been collected at Leptospermum minutifolium blossoms.

Etymology: pollinosa derives from "pollinosity", the scientific term for the microtrichose hairs covering the cuticle of some tergites in Syrphidae. This is in reference to the pollinose pleuron of Psilota pollinosa, which distinguishes it from Psilota coerulea, Psilota aislinni, and Psilota alexanderi. 
Specimens Examined: HOLOTYPE Material: AUSTRALIA: 1 km E. Forbes Creek; on flowering heath; 11.XI.2012; 874m; -35.429478 149.5028; D.J. Ferguson; CNC_Diptera255050 (1§ , ANIC). Paratypes: AUSTRALIA. Australian Capital Territory: Blundell's [Cabin], -35.292886 149.141800, 14.i.1935, Fuller, ANIC29034822 (1우, ANIC); Canberra - CSIRO Botanical Garden, -35.278878 149.108917, 26.x.1988, F.C. Thompson, USNM_ENT_00034122 (1ðð, USNM); Canberra, -35.282000 149.128686, 1-10.xii.1953, F.M. Hull, CNC_Diptera254987 (1へ̂, CNC); 18.i.1948; 28.x.1955, S.J. Paramonov, ANIC29034827; ANIC29034804; ANIC29034825 $(2 \overbrace{}^{\lambda} 1$ 우, ANIC); New South Wales: Same as holotype data, CNC_Diptera255049 (1 $\overbrace{}^{\lambda}$, ANIC); 1 miles West of Wombeyan Caves, -34.288203 149.958572, 10.xi.1955, T.G. Campbell, ANIC29034824 (1ㅇ, ANIC); 3 km South of Mount Wilson, Blue Mountains, -33.516011 150.378642, 29.xi.1984, D.K. McAlpine, B.J. Day, AustralianMuseumK408022 (1ㅇ, AM); 4 Mi N of Bateman's Bay, 35.658458 150.177744, 20.x.1953; 14.x.1952, S.J. Paramonov, USNM_ENT_00249824; ANIC2903481 (1§,1ㅇ, ANIC); 4 mile Creek, W of Wollomombi, -30.523872 152.146944, 7.xii.1961, Mackerras, ANIC29035768 (1ठへ, ANIC); Albury - Holbrook, -36.073669 146.913667, 15.xii.1949, S.J. Paramonov, ANIC29034812 (10َ, ANIC); Bald Rock National Park 25km SE Stanthorpe, 28.860783 152.055686, 1.xii.1983; 6.xii.1983; 6-7.xi.1984, D. Yeates, UQIC_Reg94655-94656; UQIC_Reg94646 (3우, UQIC); Bilpin, Blue Mountains, $33.500261150 .532989,1978$, N.W. Rodd, (Date is ambiguous reads "9.11.1978"), AustralianMuseumK410293 (1q, AM); Clarence, Blue Mountains, -33.482308 $150.224264,1982$, N.W. Rodd, Date is ambiguous reads "5.12.82", 
AustralianMuseumK410290 (19, AM); Jervis Bay, -35.035433 150.668869, 2.x.1952, S.J. Paramonov, ANIC29034809; CNC_Diptera255044 (2우, ANIC); Jinkie Creek, Blue Mountains, -33.617564 150.455908, 14.i.1992, N.W. Rodd, AustralianMuseumK408025 (1ㅇ, AM); Mannus near Tumbarumba, -35.808336 147.935786, 1.xii.1950, T.G. Campbell, ANIC29034805 (10, ANIC); Mount Tomah, Blue Mountains, -33.549614 150.416033, 1980; 16.xii.1982; 26.xi.1986; 13.ii.1984; 14.i.1985, N.W. Rodd, AustralianMuseumK407025; AustralianMuseumK410287; AustralianMuseumK406919-406920 (2ภํํ우우, AM); Mount Towinhingy near Kondos Weir, -32.856161 150.186597, 20.xi.1978, G. Daniels, GDCBReg_19691; GDCBReg_19698 (1ð1우, GDCB); Nadgee Reserve 4.7 km S Merrica Hut, -37.399378 149.908592, 29.xii.1985, E.A. Sugden, CNC_Diptera254998-254999 (2우우, CNC); Nullo Mtn 20 mls NE Rylstone, -32.737947 150.226400, 12.xi.1950, T.G. Campbell, CNC_Diptera255018 (1ðึ, ANIC); Sandy Point, Sydney, -33.926375 151.125936, 12.x.1991, R. de Keyzer, A. Sundholm, AustralianMuseumK406925 (1ㅇ, AM); South Black Range, East of Hoskinstown, -34.427778 149.534444, 1130m, 16.ii.2004; 3.iii.2004, D.J. Ferguson, ANIC29035769; CNC_Diptera255017 (1 §11우, ANIC); South Black Range, Tallaganda Nat. Pk., 15km from Hoskinstown, -35.413333 149.535000, attracted to water added to dry dam in forest, 21.ii.2004, C. Lambkin, N. Starick, ANIC29035770 (1 $ð$, ANIC); Queensland: Blue Mtn., 4 km NE Stanthorpe, SE Qld. Summit, -28.633344 151.966683, 977m, 28.ix.1987, D.K. Yeates, UQIC_Reg94654 (1へ̂, UQIC); Brisbane, -27.526975 153.040322, 10.ix.1927, J. Mann, UQIC_Reg94811 (1今, UQIC); Stanthorpe, -28.657928 151.930344, 22.x.1961, D.H. Colless, ANIC29034801 (10ે, ANIC); 3.x.1924, Frank M. Hull Collection C.N.C. 1981; 


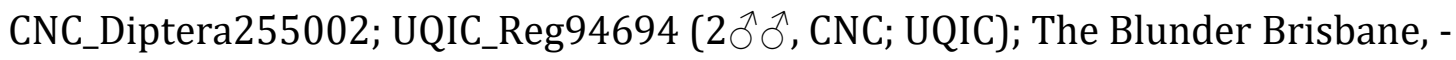
27.526975 153.040322, 18.ix.1966, C.F. Ashby, CNC_Diptera255098 (1ð^, ANIC);

South Australia: Adelaide, Belair National Park, -35.000000 138.633333, hilltopping, 4.x.1997, J.\& A. Skevington, C. Lambkin \& S. Winterton, UQIC_Reg94517 (1우, UQIC); Nat Park, 21.x.1951, E.F. Riek, CNC_Diptera253712 (1ㅇ, ANIC);

Tasmania: Hobart, -42.882139 147.327194, 15.xii.1917, G.H. Hardy,

ANIC29034802 (1 ${ }^{\lambda}$, ANIC); Victoria: Barjarg, -36.924222 145.995044; -36.924583 145.995236, 22.x.1961, D.H. Colless, CNC_Diptera255043; ANIC29034806 (2ðð, ANIC).

\section{Psilota solata Young and van Steenis sp. nov.}

(Species plate: Fig. 30, Genitalia: Fig. 41G+H, Map: 25)

Body length: 9.2-12.1 mm.

Diagnosis: Black species with orange legs. Face densely yellow pollinose, with yellow pile. Hind femur with a distinct rounded apicoventral setulose carina. Hind tibia simple. Calypter entirely brown. Tergites 3 and 4 with long, dense, tangled, bright yellow pile.

Description: Head: Head black, face densely yellow pollinose. Face yellow pilose, frons and vertex black pilose. Scape and pedicel entirely orange, postpedicel brown 
on apical half and orange below. Postpedicel oval, approximately 1.5 times as long as wide. Eye densely yellow pilose.

Thorax: Thorax black. Legs almost entirely orange, with a brown posterior stripe on the apical half of the hind femur and hind tibia brown on basal half. Hind femur with a distinct rounded apicoventral setulose carina. Hind tibia simple, with no basoventral spur. Pleuron yellow pollinose. Pile on posterior anepisternum mixed black and yellow, remainder of pleural pile yellow. Pile on scutum mixed black and white. Pile on scutellum entirely black. Wing colourless, with cells bm and cua bare on anterior half, small bare areas at the bases of cells $r_{4+5}$ and $\mathrm{dm}$. Alula completely microtrichose. Halter pale, with knob light brown. Calypter pale brown with a dark brown border.

Abdomen: Abdomen black. Abdomen entirely yellow pilose, pile on tergites 3 and 4 long, dense, and tangled. Sternites yellow, and yellow pilose.

Genitalia: Epandrium compact, about as long as tall. Cercus blunt, somewhat ovoid. Outer lobe of surstylus inserted near bottom of inner lobe, curved downwards on apical half, with apex of lobe thickened and curved slightly backwards towards base of surstylus. Inner lobe of surstylus broadening over entire length, with a slight 
ventral excavation at midlength, rounded at apex. Phallapodeme smooth. Gonostylus slightly darkened.

Discussion: This species has been collected on Leptospermum J.R. Forst. and G. Forst. (Myrtaceae) blossoms.

Etymology: solata is derived from the Latin solis, meaning "sun". This is in reference to the tangled golden pile on the abdomen of Psilota solata, which distinguishes it from all other species.

Specimens Examined: HOLOTYPE Material: Rutherford Ck., Brown Mtn., nr Nimmitabel, 25.XII.1974, G. Daniels, GDCBReg_19605, (1 $\overbrace{}^{\lambda}$, GDCB). Paratypes: AUSTRALIA. New South Wales: Bald Rock National Park, -28.860781 152.055686, on Leptospermum minutifolium blossom, 6-7.xi.1984, D.K. Yeates, UQIC_Reg9464794650; UQIC_Reg94651-94653 (1ð̋우, UQIC); Gibraltar Range National Park, Waratah track, -29.5 152.333333, on Leptospermum sp. blossom, 10-12.xi.1984, D.K. Yeates, UQIC_Reg94643 (19, UQIC); same as holotype data, GDCBReg_19606-19608 $(3+\circ, \mathrm{GDCB})$

Psilota spathistyla Young and van Steenis sp. nov. (Species plate: Fig. 31, Genitalia: Fig. 42A+B, Map: 26) 
Body length: 11.1-15.1 mm.

Diagnosis: Face black, densely grey pollinose, with black pile. Frons and vertex black pilose. Thorax black. Hind femur with a low triangular apicoventral setulose carina. Hind tibia with basoventral spur. Calypter with dark brown border. Halter pale, sometimes with knob slightly pigmented. Abdomen black. Lateral edges of tergite 3 with many long, pale pili. Male genitalia with inner lobe broadest near base, distinguishing it from the similar $P$. nigripilosa.

Description: Head: Head black, face densely grey pollinose. Face mostly black pilose with some pale pili around the anterior rim of the eye, frons and vertex black pilose. Scape and pedicel orange-brown, postpedicel brown. Postpedicel triangular, approximately 1.5 times as long as widest point. Eye densely brown pilose.

Thorax: Thorax black. Legs mostly orange, with apical $1 / 3^{\text {rd }}$ of femora, base and apex of hind tibia, and apical tarsomeres brown. Hind femur with a low, pointed apicoventral setulose carina. Hind tibia with a basoventral spur. Pleuron densely grey pollinose. Pile on anepisternum and anepimeron mixed black and white, pile on remainder of pleuron white. Pile on scutum and scutellum mixed black and whitishyellow. Wing faintly brown tinted on apical half. Wing bare on anterior $3 / 4^{\text {ths }}$ of cell bm and $1 / 4^{\text {th }}$ of cell cua, cell $c$ bare on basal $1 / 3^{\text {rd }}$. Alula completely microtrichose. 
Halter pale, sometimes with knob slightly pigmented. Calypter with border dark brown, upper calypter hairs brown, lower calypter hairs yellow.

Abdomen: Abdomen black. Tergite 2 mostly white pilose, with some central black pili at posterior border. Tergite 3 whitish-yellow pilose laterally, black pilose medially. Tergite 4 with mixed black and white pile. Sternites black, with white pilosity.

Genitalia: Epandrium compact, slightly taller than long. Cercus approximately triangular, somewhat elongate. Outer lobe of surstylus inserted near top of inner lobe, bent sharply downwards at half its total length, and then swollen at apex. Inner lobe of surstylus Phallapodeme smooth. Gonostylus darkened.

Discussion: This species has been collected on Leptospermum J.R. Forst. and G. Forst. (Myrtaceae).

Etymology: spathi- derives from the Latin spatha, meaning "spatula", and stylus is Latin for "pen" or "writing implement". This is in reference to the distinctive spatula-shaped genitalia of the male Psilota spathistyla, which can be used to distinguish it from Psilota nigripilosa. 
Specimens Examined: HOLOTYPE Material: AUSTRALIA: Tasmania: Mt Barrow; -41.366667 147.416667; 1000mm, 23.I.1992; coll.G. and A. Daniels; GDCBReg_19620; (10, GDCB). Paratypes: AUSTRALIA. Tasmania: Don River, nr Don, -41.183333 146.316667, on Leptospermum, 7.i.1992, G. and A. Daniels, Malaise trap, G. Daniels Collection, GDCBReg_19519 (1へ̂, GDCB); same data as holotype, GDCBReg_19620; GDCBReg_19617-19618 (3ððત, GDCB); Tarkine SG04, adj quarry at Browns Plain, -41.610000 145.175500, shrubland, 29.i.2015, S.J. Grove, hand collected, TMAG-F11619 (1へ, TMAG).

\section{Psilota spinifemur Young sp. nov.}

(Species plate: Fig. 32, Genitalia: Fig. 42C+D, Map: 27)

Body length: $10.3-10.8 \mathrm{~mm}$.

Diagnosis: Face black, densely white pollinose, with white pile. Frons white pilose, vertex black pilose. Thorax black. Hind femur with a distinct rounded apicoventral setulose carina, with medial side of carina entirely setulose. Hind tibia with basoventral spur. Calypter pale, with only pile at corner of upper and lower calpyter dark. Halter pale. Abdomen black, with metallic green sheen. Tergites 2,3 and 4 entirely pale pilose. 
Description: Head: Head black, face white pollinose. Face pale pilose. Scape and pedicel orange, postpedicel orange with anterior edge brown. Postpedicel oval, approximately 1.5 times as long as widest point. Eye densely brown pilose.

Thorax: Thorax black. Femora mostly brown, with only apex orange. Tarsi progressively darkened, with tarsomere 1 orange and tarsomere 5 dark brown. Legs otherwise orange. Hind femur with a distinct rounded apicoventral setulose carina, medial side of carina entirely setulose. Hind tibia with a basoventral spur. Pleuron densely grey pollinose. Pleural pile entirely white. Pile on scutum and mixed black and whitish-yellow. Pile on scutellum entirely black. Wing colourless. Wing bare on anterior $3 / 4^{\text {ths }}$ of of cell bm and $1 / 4^{\text {th }}$ of cell cua, cell c bare on basal $1 / 3^{\text {rd }}$, cells $\mathrm{m}_{4}$, $\mathrm{dm}$, and $\mathrm{r}_{4+5}$ with small bare areas near base. Alula completely microtrichose. Halter pale. Calypter pale, with pile at corner of upper and lower calypter dark brown.

Abdomen: Abdomen black, with metallic green sheen. Tergites 2 to 4 entirely white pilose. Sternites black, with white pilosity.

Genitalia: Epandrium compact, about as long as tall. Cercus approximately triangular. Outer lobe of surstylus inserted just above mid-height of inner lobe, curved downwards on apical 2/3rds, with apex of lobe thickened and curved backwards towards base of surstylus. Inner lobe of surstylus broadening greatly 
over entire length, with a ventral excavation on basal $1 / 3^{\text {rd }}$, rounded at apex. Phallapodeme smooth. Gonostylus slightly darkened.

Discussion: Nothing is known about the biology of this species.

Etymology: spini- is derived from the Latin spinus, meaning "spine, thorn, or prickle". This is in reference to the setulose carina on the hind femur of Psilota spinifemur.

Specimens Examined: HOLOTYPE Material: AUSTRALIA: New South Wales: South of Milton; -35.319178 150.439158; S.J. Paramonov; ANIC29034986; (10 ANIC). AUSTRALIA: Australian Capital Territory; New South Wales: Cotter River; 20 miles South of Milton; -35.531650 148.875125; 29.XI.1962; ANIC29034826; ANIC29034980; USNM_ENT_00249844; $(1 ð, 2$ 우우, ANIC; USNM).

Psilota tristis Klocker, 1924

(Species plate: Fig. 33, Map: 28)

Psilota tristis Klocker, 1924: 54. Type locality: Queensland, Australia.

Body length: $7.0-14.1 \mathrm{~mm}$. 
Diagnosis: Face black, pale pollinose, with dark or pale pile. Frons and vertex black pilose. Thorax black. Thoracic pile variable in colour. Hind leg simple. Calypter variable in colour. Halter variable in colour. Abdomen black. Tergite pile variable in colour.

Description: Head: Head black, face pale pollinose. Face dark or pale pilose, frons and vertex black pilose. Antenna orange to dark brown. Postpedicel variable in shape. Eye densely brown pilose.

Thorax: Thorax black. Legs mostly orange to entirely dark brown. Hind femur simple. Hind tibia simple. Pleuron densely grey pollinose to bare and shining. Pile pleuron variable in colour. Pile on scutum and scutellum variable in colour, but always at least partly black on scutellum (male only). Wing colourless. Wing microtrichia variable. Alula completely microtrichose. Halter variable in colour. Calypter variable in colour.

Abdomen: Abdomen black. Pilosity of tergites variable in colour. Sternites black, with variably coloured pilosity.

Genitalia: variable (see below). 
Discussion: Psilota tristis doubtlessly encompasses multiple species as described here. Initial DNA barcode analysis indicate that at least 19 species are represented. Further DNA barcoding and male genitalia dissection is required to adequately separate the full range of specimens into species, and will be discussed in a future revision. Furthermore, there are several other species of Psilota described from Australia that may or may not be synonyms of P. tristis, including Psilota hirta, Psilota shannoni, and Psilota queenslandica (see species not recognized below). Because it is impossible to tell what name should properly be assigned to different Barcode identities without a full revision of this complex, these species have not been treated individually.

Specimens Examined: AUSTRALIA. Australian Capital Territory: Bendora, 35.221814 149.169528, 13.ii.1952, S.J. Paramonov, ANIC29034513 (1ㅇ, ANIC) Black Mountain, -35.266303 149.095464, 24.x.1977; 13.iii.1961; 13.x.1965; 15.xii.1963; 21.ii.1966; 18.i.1966; 21.xii.1962; 26.xi.1959; 28.x.1966; 30.x.1962; 30.x.1964; 30.x.1969; xii.1987, Z. Liepa; I.F.B. Common; M. Irwin, Malaise trap; light trap, ANIC29034495; ANIC29034540; ANIC29034868; ANIC29034518; ANIC29034932; ANIC29034867; ANIC29034517; ANIC29034545; ANIC29034551; ANIC29034520-29034521; ANIC29034534; ANIC29034476; ANIC29035781 (7ふ̋̋,7웅, ANIC); Blundell's [Cabin], -35.292886 149.141800, 25.xii.1937; 11.x.1950; 24.x.1950, M. Fuller; Paramonov, ANIC29034811; ANIC29034496;

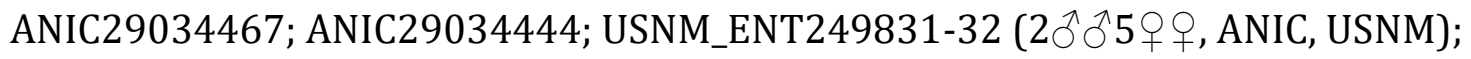


Canberra, -35.282000 149.128686, 1-10.xii.1953; , 25.xii.1985, F.M. Hull; D.C.F. Rentz, CNC_Diptera253774; ANIC29034493 (1§̋,1우,CNC, ANIC); Cotter River, 35.531650 148.875125, 23.xi.1950; 29.xi.1962, Paramonov ; Z. Liepa, CNC_Diptera253689; ANIC29034445 (2우, ANIC); Gibraltar Peak, -35.466661 148.933336, 24.xi.1948, Paramonov, ANIC29034516 (1우, ANIC); Mt. Gingera, Bancroft, -35.576694 148.779703, in swamp on Epacris, 19.i.1963, Mackerras, ANIC29035758 (1ㅇ, ANIC); Mt. Pemberton, 2km E. Gilmore, -35.416667 149.133333, dry Eucalyptus woodland, 18.x.2003; 1.xi.1947, D.J. Ferguson; S.J. Paramonov, ANIC29035754; ANIC29034832; ANIC29034878 (3 $3 \hat{\jmath}$, ANIC); New South Wales: 1 km E. Forbes Creek, SE, -35.429478 149.502800, 874m, 11.xi.2012, D.J. Ferguson, CNC_Diptera253796 (1ㅇ, ANIC); 1 km North of Guthrie's Creek, Kosciuzko National Park, -36.413086 148.375475, 1722m, 1.ii.1974, K.R. Norris, ANIC29034875 (10๊, ANIC); 1.5 km West Northwest Lansdowne via Taree, 31.780136 152.51311, Cassina blossom, 12.ix.1985, G. Williams, AustralianMuseumK410249 (1q, AM); 12 miles. N.W. Milton, -35.164567 150.298497, 487m, 5.xi.1968, D.H. Colless, ANIC29034527 (1ㅇ, ANIC); 20 miles South of Milton, -35.319178 150.439158, 30.ix.1952, S.J. Paramonov, ANIC29034835; ANIC29034876 (2ðð ANIC); 24 km W South Grafton, -29.616667 152.733333, 1.xii.1990, G. and A. Daniels, Legs removed for DNA analysis; GDCBReg_19734 (19, GDCB); 25 km W of South Grafton, -29.616667 152.733333, 5.ix.1981, G. and A. Daniels, GGDCBReg_19696 (1ð̂, UQIC); 3 km North of Lansdowne, -31.758753 152.534258, Acradenia euodiiformis, 19.ix.1993, G. Williams, AustralianMuseumK409935 (19, AM); 4 miles North of Bateman's Bay, - 
35.658456 150.177744, 14.x.1952; 12.x.1956, S.J. Paramonov; E.F. Riek, ANIC29034870; CNC_Diptera253873; CNC_Diptera253874; ANIC29034475 (3ภึ1ㅇ, ANIC); 4-10 miles North of Bateman's Bay, -35.658456 150.177744,

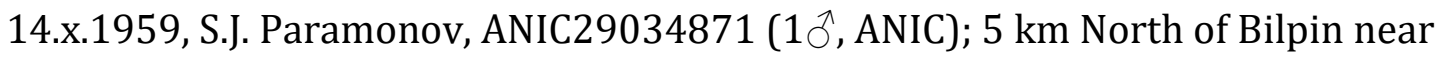
Kurrajong, -33.462750 150.572836, 1979, N.W. Rodd, N.W. Rodd Collection (Date is ambiguous reads "6.3.79"), AustralianMuseumK408029 (10^, AM); 5 mi's. NE Nerriga, -35.086408 150.170681, 27.xi.1962, I.F.B Common \& M.S. Upton, ANIC29034471 (1우, ANIC); 6 km Northeast of Bilpin near Kurrajong, -33.462750 150.572836, 1979, N.W. Rodd, Date is ambiguous reads "10.9.79", AustralianMuseumK410272-410273, AustralianMuseumK408026; AustralianMuseumK410003 (3ðð, 1†,AM); Bald Rock National Park -28.860781 152.055686, on Leptospermum minutifolium blossom, 6-1.xii.1983; 7.xi.1984, D.K. Yeates, UQIC_Reg94612-94617; UQIC_Reg94629; UQIC_Reg94742 (3ðð5우우, UQIC); Barrington Tops National Park, Polblue Swamp, -31.956389 151.435833, 18.i.2010, S.A. Marshall, debu00322900 (1ㅇ, DEBU); Barrington Tops, -31.935611 151.569264, viii.1928, M. Fuller, ANIC29034844 (1ðへ, ANIC); Batesman Bay Area, Rt. 1, -35.714256 150.179539, 13.xi.1988, F.C. Thompson, USNM_ENT_00034117 (1우, USNM); Bellmore Falls, -34.633956 150.555914, 1948, S.J. Paramonov, Date is ambiguous reads "12.11.48", ANIC29034842 (10^, ANIC); Bendalong, -35.219983 150.485778, 6.x.1968, G. Daniels, GDCBReg_19653 (1へ̂, UQIC); Berowra, 33.621622 151.150317, ANIC29035012 (1ðึ, ANIC); Bilpin, Blue Mountains, 33.500259 150.532989, 26.ix.1978, N.W. Rodd, NAustralianMuseumK408015; AustralianMuseumK410152; AustralianMuseumK408021 (3우, AM); Bimberi, - 
35.572761 148.708658, 25.xi.1934, Fuller, ANIC29034837 (1ð^, ANIC); Blackheath, 33.635592 150.283517, 17.xi.1919, G.H. Hardy, ANIC29035019 (1へ^, ANIC); 23.xi.1919, G.H. Hardy, ANIC29034548 (1ㅇ, ANIC); Bolaro State Forest, -35.700000 150.033333, 22.i.2010, M. Batley, AustralianMuseumK407883 (1ð̊, AM); Broadwater Nat. Pk., -27.357453 151.09904, 26.viii.1984, D.K. Yeates, UQIC_Reg94766 (1§, UQIC); Budawang NP, Western Distributor Road, Carter's Creek, -35.516250 150.059500, 200m, 14.xii.2003, A. Zwick, ANIC29035753 (1ðึ, ANIC); Budthingaroo Creek Kanangra Boyd National Park, -33.988542 150.088364, 26.xii.1977, G. Daniels, GDCBReg_19700 (10^, GDCB); Bullawa Ck., Mt. Kaputar Nat. Pk., -30.233333 150.1, 17.i.1994, D.K. Yeates, UQIC_Reg94680 (19, UQIC); Bungendore, -35.248714 149.450603, 27.x.1968, J.C. Cardale, ANIC29034515 (1우, ANIC); Catherine Hill Bay, -33.148439 151.617817, 12.iv.1949, S.J. Paramonov, CNC_Diptera253699; CNC_Diptera253900 (2 $\widehat{\partial} \widehat{\partial}$, ANIC); Clarence, Blue Mountains, 33.482308 150.224264, 1982, N.W. Rodd, Date is ambiguous reads "8.11.82", AustralianMuseumK410469; AustralianMuseumK408024;

AustralianMuseumK410283 (3우, AM); Clyde Mountains near Braidwood, 35.546072 149.950333, 26.x.1960; 26.x.1965, S.J. Paramonov, CNC_Diptera253713253725; ANIC29034862-29034863; ANIC29035007; USNM_ENT249829;

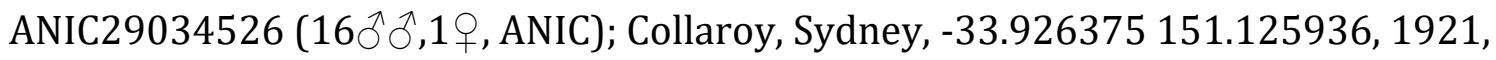
Health Dept. (Date is ambiguous reads "8.9.21"), ANIC29034858 (1ðへ, ANIC); Como, 34.004500 151.063789, 4.iv.1975, ANIC29034923 (1ㅇ, ANIC); Concord near Sydney, -33.926375 151.125936, 7.xii.1968, R. Coveney, AustralianMuseumK406924 (1ð^, AM); Conimbla National Park, -33.80547 
148.48256, 7.x.2012, M. Batley, AustralianMuseumK410608 (19, AM); Coocumbac Island Nature Reserve, Taree, -31.920678 152.454450, Floodplain forest landing near small pool of water on fallen log, 18.i.1994, G. \& T. Williams, AustralianMuseumK410628 (1우, AM); Deep Ck., Narrabeen, -33.719681 151.297125, 28.xii.1961, D.H. Colless, ANIC29034514 (1우, ANIC); Duffy's Forest, near Sydney, -33.926375 151.125936, 14.xi.1970, D.K. McAlpine, AustralianMuseumK410117; AustralianMuseumK410132; AustralianMuseumK410140; AustralianMuseumK410254; AustralianMuseumK410294 (1ð, 4우, AM); Durras Bay, -35.645003 150.291439, 12.xi.1953; 29.ix.1959, Z. Liepa; F.M. Hull, CNC_Diptera255056-255057,

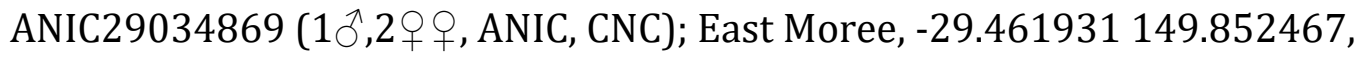
14.xii.1961, E. Cheah, CNC_Diptera245382 (19, MCZ); East of Taree, Manning Point, -31.894431 152.658919, littoral rainforest on Acmena smithii blossom T.I., 8.xi.1990, G. Williams, AustralianMuseumK410238 (1ðð, AM); Gibraltar Range National Park, Waratah track, -29.5 152.333333, on Leptospermum sp. blossom, 1012.xi.1984, D.K. Yeates, UQIC_Reg94568 (1우, UQIC); Grose Vale, -33.584000 150.674000, 1978, N.W. Rodd, AustralianMuseumK410271 (1へર, AM); Gundamaian National Park, -30.511594 152.995342, viii.1962, U.Q.I.C. Loan \#1036, JSM5623, UQIC_Reg94562 (1ð, UQIC); Heathcote, -34.083333 151.016667, 31.x.1948,

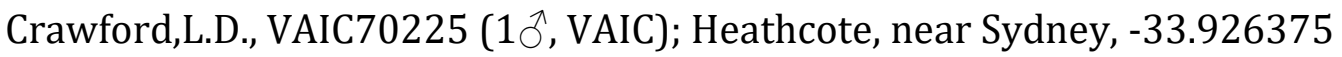
151.125936, 18.xi.1970, D.K. McAlpine, AustralianMuseumK410141 (10َ, AM); Hornsby, -33.704900 151.099011, 20.xi.1932, Darlington, Australia, CNC_Diptera253779 (1우, CNC); Jervis Bay, -35.035431 150.668869, 1.x.1952; 
18.i.1949; 18.ix.1949; 2.x.1952; 18.ix.1951; 24.i.1963; 4.ix.1951, Paramonov; T.G. Campbell; I. Liepa; Z. Liepa, ANIC29034450; CNC_Diptera253870; ANIC29034505; USNM_ENT249830; ANIC29035003; ANIC29034877; USNM_ENT249826; CNC_Diptera253888 (5ð⿱ð 2 우우, ANIC,USNM); Katoomba, -33.713131 150.313267, 10.x.1953; 14.ii.1952; 4.xii.1952; 20.i.1921; 31.xii.1956, F.M. Hull; G.H. Hardy, CNC_Diptera253773; AustralianMuseumK410587; Jeff_Skevington_Specimen3040930410; AustralianMuseumK407101; CNC9032 (3 $\widehat{\jmath}, 3$ 우우, CNC, AM, MCZ); Killara, 33.768642 151.163478, 1.ix.1935, M.F. Day, ANIC29034873 (1§^, ANIC); Kosciusko, -36.455897 148.263600, F.H. Taylor, ANIC29034841 (1 万人, ANIC); Kosciuszko National park, 3.2km WSW of Thredbo, near Dead Horse Gap, -36.520833 148.268333, 1496m, over narrow stream in flowering grassland, 1-11.i.2002, C.L. Lambkin, N.T. Starick, ANIC29007126-27 (2우, ANIC); Ku-ring-gai Chase Nat. Park, -33.637567 151.233900, 2.x.1972, A. and G. Daniels, GDCBReg_19726 (1ð^, GDCB); Loftus, near Sydney, -33.926375 151.125936, 7.xi.1970, D.K. McAlpine, AustralianMuseumK410126; AustralianMuseumK410143; AustralianMuseumK410165 (3우, AM); Lorien Wildlife Refugee, 3 km North of Lansdowne, -31.758753 152.534258, Acradenia euodiiformis blossoms, 25.ix.1991, G. Williams, AustralianMuseumK407176 (1ㅇ, AM); Mellong Ck, 12.8km S of Putty, 33.050000 150.683333, 23.xi.1978, G. Daniels, Daniels Collection, GDCBReg_19660 (1우, GDCB); Mooney Mooney Creek, near Gosford, -33.437894 151.246467, 3.ix.1984, D.K. McAlpine, B.J. Day, AustralianMuseumK405939 (1ðત, AM); Mount Kaputar National Park, near Dawsons Springs, -30.284722 150.167500, 1.i.2010, S.A. Marshall, debu00318889 (1ㅇ, DEBU); Mount Keira, near Wollongong Uni Obser, 
-34.402692 150.856469, 25.ix.1992, M. Gregory, White label "30",

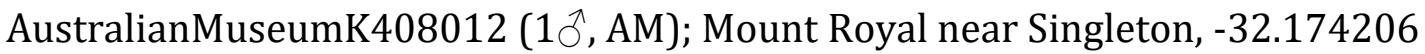
151.326167, 12.xi.1983, B.J. Day, K.C. Khoo, AustralianMuseumK410616 (1ð , AM); 13.xi.1983, B.J. Day, AustralianMuseumK408018 (1우, AM); Mount Tomah, Blue Mountains, -33.549614 150.416033, 19.x.1988, N.W. Rodd, N.W. Rodd Collection, AustralianMuseumK410281 (1ㅇ, AM); Mount Victoria, -33.583333 150.250000, 29.ix.1934, D.F.W., ANIC29034859 (1ð^, ANIC); Mt. Kaputar N.P., Bullawa Ck., -30.14 150.06, 16-19.i.1994, M.E. Irwin, D.K. Yeates, malaise trap, UQIC_Reg94676-94678

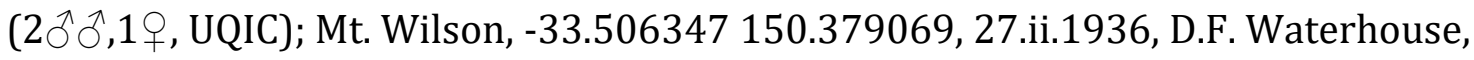
ANIC29034523 (10, ANIC); N.E. [New England] National Park, near Entrance, 30.476022 152.660181, 29.xi.1960, C.W. Frazier, ANIC29035006 (1§ , ANIC); Nadgee NR. near Eden, -37.399378 149.908592, wet sclerophyll forest, 1415.ii.1986, D.J. Bickel, AustralianMuseumK410253 (1ㅇ, AM); Narrabeen, 33.719681 151.297125, 15.ix.1934, D.F.W., CNC_Diptera253700-253701 (2仓ึへ, ANIC); Nelligen, -35.645156 150.092364, 16.i.1970, J.C. Cardale, ANIC29034925 (1우, ANIC); Norfolk Island, -29.040836 167.954711, 30.xi.1971, D. Kruger, ANIC29034482 (1우, ANIC); Northbridge, Sydney, -33.926375 151.125936, 23.viii.1932, Fuller, ANIC29034451 (1 9 , ANIC); Nullo Mountains, 20 miles NE Rylstone, -32.737947 150.226400, 11.xi.1950, T.G. Campbell; Paramonov, ANIC29034506; ANIC29034469; ANIC29034497 (3우, ANIC); O'Hares Creek, Darkes Forest, -34.229969 150.908403, 2.ix.1992; 23.ix.1992, M. GregoryAustralianMuseumK408017, AustralianMuseumK408010;

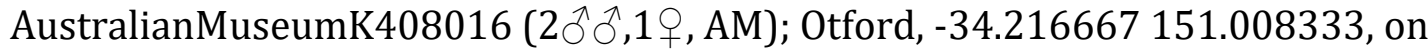


Acmena smithi flowers, 25.xi.1995, A. Sundholm, A. Scott, ANIC29035778 (1ð, ANIC); Peats ridge, -33.333333 151.233333, on flowers, Leptospermum, 23.ix.1995, A. Sundholm, ANIC29035759 (1ㅇ, ANIC); Petersham, -33.896100 151.154300, x.1927, M. Fuller, ANIC29035016 (1ð, ANIC); Port Macquarie, -31.433356 152.899886, 26.ix.1981, D.K. McAlpine \& B.J. Day, AustralianMuseumK410124; AustralianMuseumK410618 (1へ,1, AM); Roseville, Sydney, -33.926375 151.125936, 1915, Date is ambiguous reads "3.1.15", ANIC29035023 (1 ${ }^{\AA}$, ANIC); Royal National Park, -34.114342 151.046586, 28.xii.1970, D.K. McAlpine, AustralianMuseumK410620 (1ð, AM); on leaves of Xanthorrhoea, 4.xii.1970, D.K. McAlpine, AustralianMuseumK410256 (1ㅇ, AM); Ruby Creek, near Stanthorpe, 28.608586 152.010250, 4-7.xi.1969, E.C. Dahms, Possibly in Queensland, QM_REG._NO._T223217 (10, QM); Rutherford Ck., Brown Mtn, nr. Nimmitabal, 36.600000 149.416672, 24.xii.1974, G. Daniels, GDCBReg_19643 (1 $\delta^{\lambda}$, UQIC); SYDNEY, -33.926375 151.125936, 10.x.1948, Crawford,L.D., Collected Sydney University campus, VAIC70224 (1ð, VAIC); Siding Spring Observatory, -31.272222 149.061111, summit, 17.x.1997, C. Lambkin, J. Skevington \& S. Winterton,

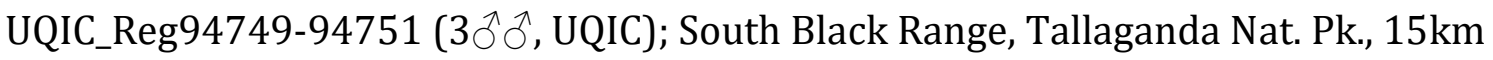
from Hoskinstown, -35.413333 149.535000, flowering heath, 21.ii.2004, C. Lambkin, N. Starick, ANIC29035777 (10^, ANIC); South Black Range, east of Hoskinstown, -35.411111 149.534444, 1130m, 31.xii.2010, D.J. Ferguson, CNC_Diptera253795 (1ㅇ, ANIC); Summit, Mount Gower, Lorde Howe Island, 31.588175 159.073681, 19.ii.1971, D.K. McAlpine, AustralianMuseumK407879 (19, AM); Sydney, Terry Hills, -33.926375 151.125936, 14.ix.1963, D.H. Colless, 
ANIC29034872 (1ð, ANIC); Sydney, -33.926375 151.125936, 16.x.1918; 24.xi.1918;

24.ix.1922; 8.ix.1921, G.H. Hardy; Health Dept; Deane \& G. Mocatta, ANIC2903454142; ANIC29034857; ANIC29034549; Jeff_Skevington_Specimen30406;

UQIC_Reg94597 (3ðึฎ,3우, ANIC, QM); Tibooburra, Cobham Lake, -30.146856 142.087394, 17.xi.1949, S.J.K. Paramonov, ANIC29034937 (1ðへ, ANIC); Toronto, 33.016194 151.582583, ANIC29034504; ANIC29035020 (1ðð,1우, ANIC);

Wambelong Ck, Warrumbungle NP, -31.323000 149.027000, Malaise across creek bed, 21.i-9.ii.2009, S.L. Winterton, Malaise trap, CNC661779-661782; CNC669607

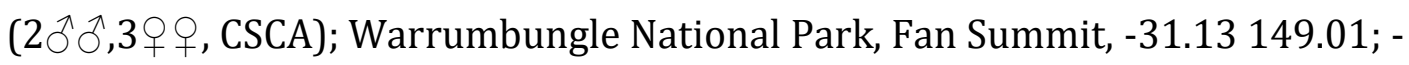
31.130000 149.010000, hilltop, 27.iii.1999, S.A. Marshall, CNC_Diptera254194254197 (4ð, DEBU); Warrumbungle National Park, -31.2743 148.989427, 17.x.1997, J. Skevington, S. Winterton, C. Lambkin, malaise trap, UQIC_Reg94755 (19, UQIC); Waterfall, -34.141986 150.992144, 30.xii.1933, Fuller, CNC_Diptera253703 (1우, ANIC); Winburndale Nat. Reserve, NE. of Bathurst, 33.333164 149.789450, 6.xii.1974, J. Cardale, ANIC29034501 (1, ANIC); Woy Woy, -33.489839 151.319942, 8.ix.1924, ANIC29034533 (19, ANIC); [Royal] National Park, Gundamaia, -34.083944 151.103617, 23.viii.1925, Mackerras, ANIC29034535; ANIC29034536 (2q, ANIC); [Royal] National Park, Sydney, -33.926375 151.125936, 4.x.1925; 13.x.1948; 18.xi.1955, Paramonov; D.K. McAlpine, ANIC29034547; ANIC29034443; CNC_Diptera253884; CNC_Diptera253889-253891;

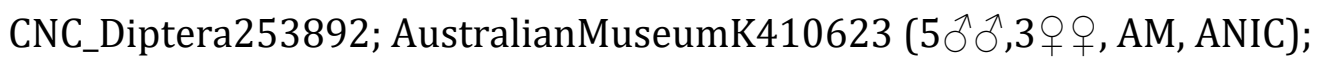
approximately 24 km North Northwest Gloucester, -31.795028 151.949758, Acmena smithii rainforest, 5.xii.1990, G. \& T. Williams, T.2., AustralianMuseumK410246 (1온 
AM); near Braidwood, -35.434689 149.802942, 31.x.1951, S.J. Paramonov,

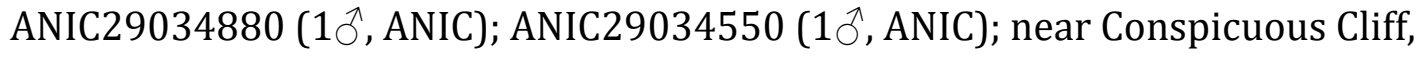
South of Nornalup, -35.004314 116.880339, fw. cr. behind dunes, 7.xi.1991, D. Bickel, AustralianMuseumK407910 (1우, AM); Northern Territory: 17 km North of Pine Creek, -13.666058 131.836867, on Eucalyptus foelcheana, 22.xi.1974, E.M. Exley \& R.I. Storey, UQIC_Reg94759 (19, UQIC); Bill's Point, Mary River, -12.932286 131.809983, 3.viii.1929, I.M. Mackerras, T.G. Campbell, ANIC29034904 (1ðへ, ANIC); Gregory Nat'l Park, Humbert Track 1.1km S Bullita Camp Ground, -16.213056 130.419722, nr weeping Boab tree, 5-12.vi.2001, M.E. Irwin, F.D. Parker, C. Lambkin, CNC_Diptera255042; ANIC29035774 (2우, ANIC); Queensland: $10 \mathrm{~km} \mathrm{~N} \mathrm{of}$ Dunwich, Stradbroke Island, -27.412264 153.440086, 19.ix.1981, G. and A. Daniels, GDCBReg_19749 (19, GDCB); 14 km W by N of Hope Vale Mission, -15.294200 145.108236, 10.x.1980, D.H. Colless, ANIC29034449 (1ㅇ, ANIC); 15km N. Dalveen, 28.351623 151.972712, on Angophora costata, 13.i.1981, J. \& C.R. King, QM_REG._NO._T221284 (19, UQIC); 18 km South of Ravenshoe, -17.771204 145.485042, 9.ix.1983, N.W. Rodd, AustralianMuseumK410131 (1ð, AM); 2 km W of Rainbow Beach, -25.966667 153.150000, rainforest, 24.ix.1994, G. \& A. Daniels \& C.J. Burwell, GDCBReg_19576; GDCBReg_19578-19579; GDCBReg_19581;

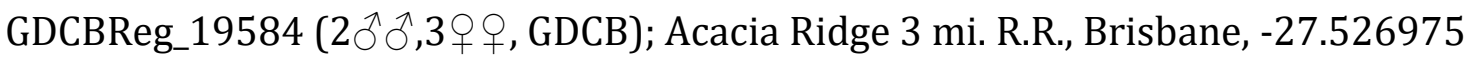
153.040322, 26.ix-3.x.1953; 12.ix.1967, F.M. Hull; E.C. Dahms, CNC_Diptera253764;

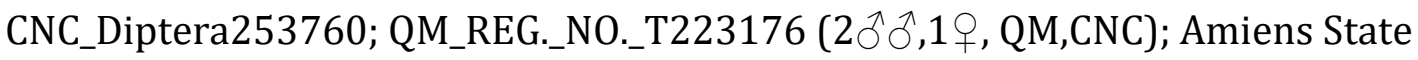
Forest, nr. Stanthorpe, -28.606395 151.799206, 24-26.xi.1981, G. Daniels \& M.A. Schneider, UQIC_Reg94895 (1ðð, UQIC); Binna Burra, Lamington National Park, - 
28.195125 153.188792, 700m, 29.x.1986, I.F.B. Common, ANIC29035782 (1ðึ, ANIC); Blue Mtn., 4 km NE Stanthorpe, SE Qld. Summit, -28.633343 151.966683, 977m, 28.ix.1987, D.K. Yeates, UQIC_Reg94627 (10̂, UQIC); Bluff Range, Biggenden, 25.600000 152.050000, 7-10.x.1972; 18.viii.1972, H. Frauca, ANIC29034555; ANIC29034997 (1ð^,1ㅇ, ANIC); Bribie Island, -26.9861 153.132525, 26.ix.1984; 29.viii.1918, G. Daniels; H. Hacker, UQIC_Reg94892; QM_REG._NO._T223187 (2ððð’ QM); Brisbane Forest Pk., -27.416667 152.833333, 9.ix.1995, M.A. Schneider, UQIC_Reg94772 (1ðึ, UQIC); Brisbane, Mount Coot-tha, -27.483333 152.950000, 170m, hilltop, 19.ix.2014; 9.ix.1972; 10.viii.1997, J.H., A.M. and A.W. Skevington; A.M. Hemmingsen; J. \& A. Skevington \& S. Winterton, hand collected, CNC451769;

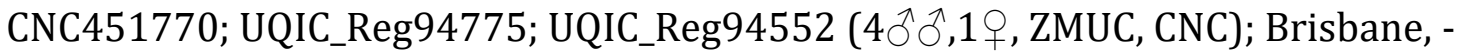
27.526975 153.040322, 15.ix.1913; 1.i.1927; 11.ix.1965; 18.ix.1965; 21.ix.1963; 18.ix.1914; 20.ix.1916; 20.viii.1918; 2.ix.1927; 22.xii.1953; 25.ix.1925; 29.viii.1916; 7.ix.1927, J. Mann; C.F. Ashby; S.J. Paramonov; F.H. Roberts; H. Hacker; G.H. Hardy; L. Pottenger, UQIC_Reg94815-94818; UQIC_Reg94820; ANIC29034553; ANIC29034855; ANIC29034828; ANIC29034530; ANIC29034538-29034839; QM_REG._NO._T223167; QM_REG._NO._T223170-223172; QM_REG._NO._T223178223183; QM_REG._NO._T223190; ANIC29034554; AustralianMuseumK407687; AustralianMuseumK407611; AustralianMuseumK407221-407222; AustralianMuseumK407226-407229; AustralianMuseumK407233-407236; AustralianMuseumK407239; AustralianMuseumK407566-407568; AustralianMuseumK407570-407572; AustralianMuseumK407574-407578; AustralianMuseumK407583-407592-407594; AustralianMuseumK407596-407599; 
AustralianMuseumK407601; AustralianMuseumK407603-407604;

AustralianMuseumK407606; AustralianMuseumK407612;

AustralianMuseumK407685; AustralianMuseumK407693;

AustralianMuseumK407696; AustralianMuseumK407699-407702;

AustralianMuseumK407705; AustralianMuseumK407709-407711;

AustralianMuseumK407714-407716; UQIC_Reg94809;

JeffSkevingtonSpecimen\#31370-31371; Jeff_Skevington_Specimen30416

$(42 \precsim ð 45$ 우, $\mathrm{AM}, \mathrm{ANIC}, \mathrm{MCZ}, \mathrm{QM})$; Brown Lake, N. Stradbroke Is., -41.345000

147.352778, 21-24.ix.1984, D. Yeates, QM_REG._NO._T221276 (19, QM); Bunya Mountains, -26.884475 151.597200; -26.886078 151.596803, 1925, H. Hacker, Date is ambiguous reads "10.12.25"; QM_REG._NO._T223511; QM_REG._NO._T223169 (2ㅇ, QM); Caboolture, -27.066672 152.966667, 25.viii.1959, F.A. Perkins, QM_REG._NO._T222171 (19, QM); Cairns District, -16.918678 145.777725, Dodd, ANIC29034491 (1오, ANIC); Caloudra, -26.799000 153.133000, 17.viii.1934; 1.ix.1940; 23.viii.1933; 5.viii.1932, F.A. Perkins; C.F. Ashby; J.K. Guyomar, QM_REG._NO._T221280; ANIC29034529; CNC_Diptera253775; UQIC_Reg94715; ANIC29034850 (4ðð, 1우, ANIC, CNC, QM); Camira, nr. Ipswich, -27.634903 152.915636, 12.ix.1981, G. Daniels, GDCBReg_19733 (1へર, UQIC); Camp Milo, Cooloola, -26.043286 153.033894, 15-18.x.1978, G.B. Monteith, QM_REG._NO._T223177 (19, QM); Carnarvon National Park, Mount Moffatt Summit, -25.059722 148.043889, 1097m, 29.xi.1997, J. Skevington, C. Lambkin, QM_REG._NO._T221267 (1ㅇ, UQIC); Carnarvon National Park, Sugarloaf Mountain Summit, -24.898611 147.778611, 1.xii.1997, J. Skevington, C. Lambkin, 
QM_REG._NO._T221279 (10^, UQIC); Carnarvon National Park, “Fly Hill” summit near West Branch Camp , -24.971167 147.989000, 900m, 12.x.2002, J. Skevington, CNC_Diptera294990 (10̄, CNC); Cleveland, -27.533333 153.266667, viii.1924, G. Daniels, AustralianMuseumK407602 (1 + , AM); G.H. Hardy, AustralianMuseumK407708 (1ㅇ, AM); Cooloola, -26.043286 153.033894, 1728.viii.1912, E.C. Dahms, light trap, open forest, QM_REG._NO._T223185 (1へ^, QM); Culgoa Floodplain NP, Reedy Swamp, 7km NW Toulby Gate -28.915722 146.976925, 138m, 18.xi.2009, C. Lambkin, N. Starick, hand net, CNC_Diptera255048; QM_REG._NO._T222142; QM_REG._NO._T222151 (3ㅇ, QM); Currumbin, 28.136000 153.483000, 8.iv.1954, K.R. Norris, ANIC29034490 (19, ANIC); D'Aguilar National Park, Mount Glorious, Greene's Creek, -27.323522 152.762033, 9.xi.2014, J.H., A.M. and A.W. Skevington, CNC385040-385041 (2 9 , CNC); D'Aguilar National Park, Mount Glorious, -27.327778 152.753611, 687m, rainforest, 15-20.ix.2014, Malaise trap, CNC457517 (1ㅇ, QM); Daintree National Park, Cape Tribulation area, 15.117622 145.076950, 5.xii.2014, J.H., A.M. and A.W. Skevington, CNC385020385021 (2우, CNC); Dayboro, 0.5 km South, -27.200000 152.816667, 1819.ix.1999, S.G. Evans, QM_REG._NO._T223220; QM_REG._NO._T223231-223232

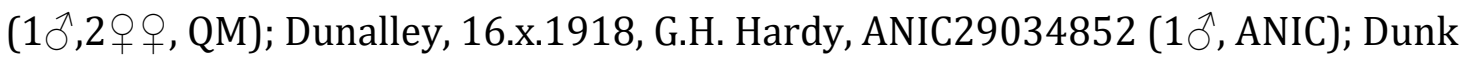
Island, -17.944558 146.153289, viii.1977, G.H. Hardy, AustralianMuseumK407595 (1ठ, AM); Durham Downs- Nokandra, -26.116933 149.076992, 13.xi.1949, S.J. Paramonov, ANIC29034938 (19, ANIC); Eungella National Park, Chelmer's Road, 21.132822 148.492683, 19.xii.2014, J.H., A.M. and A.W. Skevington, CNC451694;

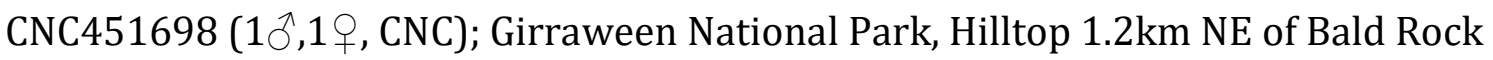


Campground, -28.821942 151.937850, 31.xii.2014, J.H. Skevington, CNC451707;

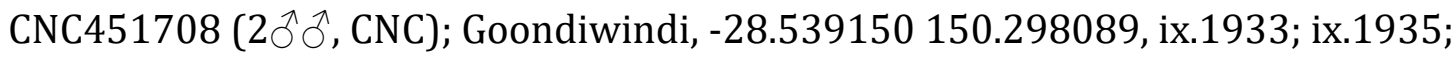

ix.1953; ix.1965, G.H. Hardy, AustralianMuseumK407607;

AustralianMuseumK407694; AustralianMuseumK407610;

AustralianMuseumK407712; AustralianMuseumK407717-407719 (6ðð,2우, AM);

$(1 \overbrace{}^{\lambda}, \mathrm{AM})$; Gordonvaie, summit of Walsh_Pyramid, -17.130158 145.800111,

13.vi.1998, M. Polak, CNC_Diptera255047 (1ㅇ, CNC); Illaweena St, Drewvale, -

27.643333 153.063333, 40m, scribbly gum/heath, 9.ix.2003,

QM_REG._NO._T222137-222138 $\left(1{ }^{\Uparrow}, 1\right.$ 우, QM); Kuranda, -16.816878 145.633497,

F.P. Dodd, ANIC29034981 (1 ${ }^{\lambda}$, ANIC); Lake Broadwater 25 km SW Dalby, -

27.350439 151.096633, on Leptospermum flavescens blossom, 18.x.1985, D. Yeates,

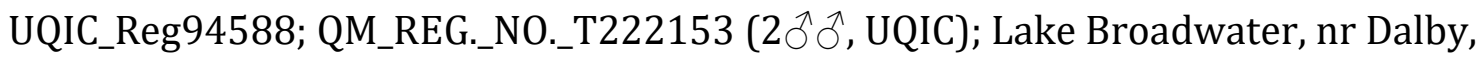

Qld site A, -27.350000 151.100000, 28.ix.1986, G. and A. Daniels, GDCBReg_19516

(10ิ, UQIC); Lamington National park, -28.210000 153.127000, iii.1921; 23.i-

3.ii.2009, G.H. Hardy; G. Monteith \& F. Turco, QM_REG._NO._T222139;

QM_REG._NO._T223164-223165 (3우, QM); Lookout, Clark Ra., Cathu State Forest, 20.822833 148.541667, 820m, 17.i.1995, G. and A. Daniels, GDCBReg_13079;

GDCBReg_19572-19573 (3ð̋, UQIC); Mahogany Forest, Mount Moffat National Park, -24.933333 148.066667, 1200m, 25.ii.1996, C.J. Burwell, S. Evans, light trap, QM_REG._NO._T222136 (19, QM); Maleny, -26.762631 152.852242, 1965, C.F. Ashby, ANIC29034861 (10 , ANIC); McIlwraith Ra. 8km NE Coen, -13.888333 143.253500, 540m, rain forest, 7.i.1995, G. and A. Daniels, GDCBReg_13077-13078 (2우우, GDCB); Moreton Island, Mount Tempest, -27.150000 153.400086, 285m, 
hilltopping, 21.ix.1997, J.\&A. Skevington, CNC483880 (10ิ, CNC); Mossman, N.Q., 16.450000 145.373333, Raqment, Name and date unclear, CNC_Diptera253777 (1우, CNC); Mount Elna, Summit, -23.159722 150.451667, 284m, 19.ix.1999, J.\&A. Skevington, QM_REG._NO._T221278 (1ð, UQIC); Mount Greville near Aratula, 28.083333 152.500000, 5.xii.1996, C.J. Burwell, S. Evans, QM_REG._NO._T223210

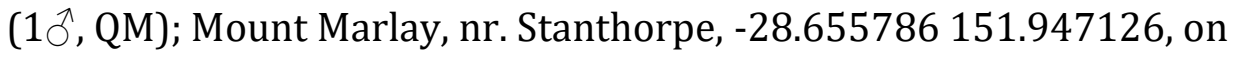
Leptospermum; 22.ix.1985; 7.x.2002, D.K. Yeates, UQIC Loan UQIC_Reg94669; UQIC_Reg94736-94738; UQIC_Reg94732; ANIC29035755-57; UQIC_Reg94579;

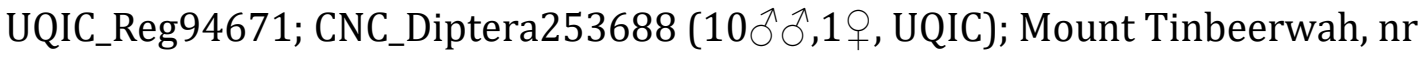
Cooroy, -29.950000 152.433333, 29.ix.1985, G. and A. Daniels, GDCBReg_1955919560 (2우우, GDCB); Mount Walsh National Park, Bluff Range Biggenden, 25.567886 152.048761, 762m, 12.viii.1972; 4.x.1976, H. Frauca, CNC_Diptera253885, ANIC29034998; ANIC29035000; ANIC29035004 (4へ̋ð゙, ANIC); Mount Walsh summit, -25.583333 152.050000, 640m, open forest \& rock slabs, 23.xii.1998, C.J. Burwell, S.G. Evans, QM_REG._NO._T223218 (10ึ, QM); Mt Macartney, Cathu State Forest, -20.834333 148.537333, 850m, rain forest, 17.i.1995, G. and A. Daniels, GDCBReg_13076 (1ㅇ, GDCB); Mt. Coot-tha Reserve, Summit Lookout, 27.4847629 152.9591569, 19.ix.2014, J.H., A.W., \& A.M. Skevington, CNC372634372635 (2ðð, CNC); Mt. Glorious, -27.333422 152.766644, 19.xii.1962; 25.iv.1930, C.F. Ashby, ANIC29034473-74; CNC_Diptera253864; QM_REG._NO._T221270 (4우, ANIC, QM); Mt. Walsh Nat. Park, Mt. Walsh summit, -25.573333 152.051389, 23.xii.1998, C. Lambkin, D. White, D. Yeates, S. Evans, C. Burwell, QM_REG._NO._T221262-221264 (3ððへ, UQIC); Mt. Walsh, nr. Biggenden, -25.567886 
152.048761, 1000m, 8.x.1976, H. Frauca, ANIC29034499; ANIC29034507;

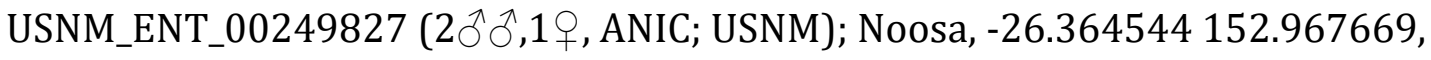
1969, J.K. Guyomar, Date is ambiguous reads "4.10.69", ANIC29034864 (10^, ANIC); Northeast of Coongara Rock, Ban Ban Range, Southwest Biggenden, -25.512597 152.037756, 24.viii.1976, H. Frauca, ANIC29035013 (1ð, ANIC); Nr. Rosedale, 24.631128 151.913664, 27.x.1974, H. Frauca, ANIC29034441; ANIC29034508 (2ㅇ, ANIC); on blossoming dogwood, Jacksonia scoparia, 27.x.1974, ANIC29034466 (1ㅇ, ANIC); Oakwells site 7, $-25.806667148 .271667,580 \mathrm{~m}$, stock through, partly cleared gully, brigalow, vine scrub, 1.x.2005, C. Eddie, $(1 \widehat{\jmath}, \mathrm{QM})$; Orientos - Nappamerrie, 27.600878 141.106858, 5.xi.1949, S.J. Paramonov, ANIC20934902;

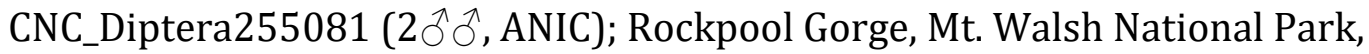
Biggenden, -25.605889 152.027519, 4.x.1976, H. Frauca, ANIC29034470 (1의, ANIC); Rockwood Station, 23 km E. Condamine, S.E.Q., -26.415218 150.917483, 18.viii.1984, D. Yeates, UQIC_Reg94767 (19, UQIC); Sheoak Ridge Nature Reserve near Julatten, -16.645258 145.403367, 9.xi.2014, C. Baker, M. Achatz, Malaise trap, CNC457380 (1ㅇ, CNC); Six Mile Creek, W of Elimbah, -27.016667 152.933333, 15.ix.1985, G. and A. Daniels, GDCBReg_19570 (1へ, UQIC); Stanthorpe, -28.657928 151.930344, 14.x.1923, 613, ANIC29034846 (1, , ANIC); $30 . i x .1925$ UQIC_Reg94760; CNC_Diptera253727 (2ððð, UQIC; ANIC); Stockyard Creek, SW of Capalaba, -27.550000 153.166667, 25.ix.1982, G. Daniels, GDCBReg_19669 (1우, UQIC); Sunnybank, -27.578997 153.059006, 1950, I.M. Mackerras, Date is ambiguous reads "3.9.50", ANIC29034845 (10^, ANIC); Tambourine Mountain, 27.923086 153.171717, 18.ix.1914, H. Hacker, QM_REG._NO._T223168 (1우, QM); 
Tibro Creek, -26.933333 152.950000, 26.viii.1960, F.A. Perkins, QM_REG._NO._T221266 (19, UQIC); Tibrogargan Ck., Glasshouse, -26.933333 152.950000, 29.viii.1958; 10.ix.1957, I.C. Yeo; F.A. Perkins, QM_REG._NO._T221271222172; UQIC_Reg94723 (1§^, 2우우, UQIC); Tin Can Beach, -25.919297 153.003278, on Leptospermum sp., 21.viii.1957, F.A. Perkins, QM_REG._NO._T221281 (1ð, UQIC); Toorbul Pt., -27.033333 153.100000, 11.ix.1952, CNC_Diptera255054 (1へ̂, UQIC); Tully-Yabulu Hwy. 1, -18.780303 146.126394, 29.viii.1986, E.A. Sugden, car net, Jeff_Skevington_Specimen43813 (19, UQIC); Walten, -23.580000 149.130000, M. Taylor, ANIC2903460 (1, ANIC); Warrumbungle National Park; Observatory, 31.274727 149.066153, Hilltop, 17.x.1997, J. Skevington, C. Lambkin, UQIC_Reg94753-94754 (2ððત, UQIC); West Bluff Range Hictden Canyon via Biggenden, -25.600000 152.050000, 22.viii.1975, H. Frauca, ANIC29034999 (1ðへ, ANIC); West Claudie River, 4 km SW road junction, -12.733333 143.25, 1.xii.1986, G. Daniels \& M.A. Schneider, malaise trap, UQIC_Reg94641 (19, UQIC); Wild Horse Mountain nr Beerburrum, -26.916667 153.000000, 12.iv.1986; 28.ix.1985, G. and A.

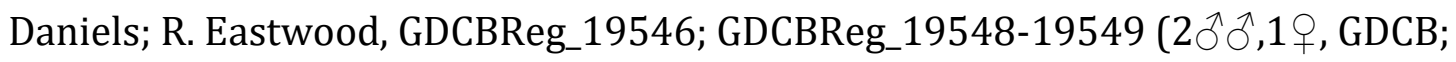
Woombye, nr. Nambour, -26.666667 152.966667, 11-16.x.1965, D.H. Colless, ANIC29034528 (1, ANIC); Yarraman, 2.2km SSE, -26.857000 151.994000, 28.iii10.iv.2010, G. Monteith, malaise trap, QM_REG._NO._T222150 (19, QM); nr. Lake Wabby, Fraser Island, -25.457644 153.129211, 9.viii.1981, M.A. Schneider, UQIC_Reg94758 (10̄, UQIC); rainforest nr. Rainbow beach, -25.897878 153.090378, rainforest, 14.xi.1982, G. and A. Daniels, GDCBReg_19671 (19, UQIC); South Australia: Adelaide, Belair National Park, -35. 138.633333, hilltop, 4.x.1997, J.\&A. 
Skevington, C. Lambkin, S. Winterton, Jeff_Skevington_Specimen4413;

UQIC_Reg94774 (2へ̂̉, UQIC); Ferguson, ANIC29034532 (1우, ANIC); M[oun]t Lofty area near Adelaide, -35. 138.733333, 4.x.1997, J.\&A. Skevington, C. Lambkin, S. Winterton, hand collected, Jeff_Skevington_Specimen1246;

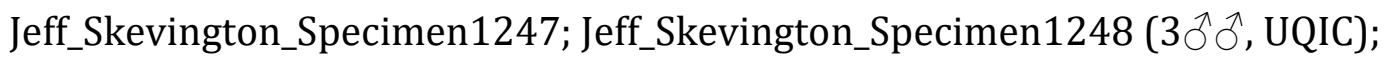
Wyperfeld NP, East Lookout, -35.594192 142.112061, 21.x.2014, J.H., A.W., \& A.M. Skevington, A.D. Young, A. Wyatt, \& S. Namek, CNC384997 (1へ̂, CNC); Tasmania: 1 mile South of Scamander, -41.501533 148.248764, 18.i.1948, Key Carne \& Kerr, ANIC29034935 (1ㅇ, ANIC); 4 miles. E of Miena, -41.991797 146.780253, 26.i.1948, Key, Carne, \& Kerr, ANIC29034500 (1ㅇ, ANIC); 6 km S Deloraine, -43.583333 146.650000, 12.i.1992, G. and A. Daniels, GDCBReg_19624 (1ㅇ, GDCB); Advent Bay, 25-28.xii.1922, A. Tonnoir, ANIC29035779-80; ANIC29034543; CNC_Diptera253894; CNC_Diptera253895; CNC_Diptera253896;

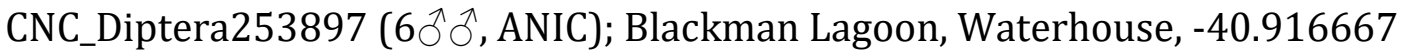
147.583333, on Banksia marginata, 18.i.1992, G. and A. Daniels, GDCBReg_19520 (1乞ึ, GDCB); Cradle Mountain NP Lake Windemere, -41.683478 145.953303, 1000m, M.P. van Zuijen, CNC_Diptera253785 (1우, CNC); Cradle Mountain NP Pelion gap, 41.683478 145.953303, 1080m, 28.i.2006, M.P. van Zuijen; W. van Steenis, CNC_Diptera253786-253787 (2우우, CNC); Cradle Mountain NP, Waterfall Valley, 41.683478 145.953303, 1000m, 26.i.2006, M.P. van Zuijen, CNC661784-661785 (2ㅇ, WVSPC); Cradle Mountain National Park, Lake Windmere, -41.767125 145.956424, 1000m, 26.i.2006, M.P. van Zuijen, Jeff_Skevington_Specimen43818 (1ㅇ, UQIC); Cradle Mountain National Park, Pelion Gap, -41.864589 146.058961, 
1080m, 28.i.2006, M.P. van Zuijen, Jeff_Skevington_Specimen43819 (1ㅇ, UQIC);

Cradle Mountain National Park, Pencil Pine Creek, -41.683478 145.953303, 20.ii.1994, J. \& A. Leask, AustralianMuseumK410097; AustralianMuseumK410479 (2웅, AM); Eaglehawk Neck, -43.019722 147.925000, G.H. Hardy, Date is ambiguous reads "23 4.16/28", AustralianMuseumK407605 (1ㅇ, AM); Elizabeth Town, -41.466667 146.566667, 22.x.1997, A.I. Knight, QM_REG._NO._T223230 (1ठ઼, QM); Flinders Island: Wingaroo flats: dragon lagoon, -39.869400 147.967000, on flowering Leptospermum, 26.iii.2014, S. Grove, CNC_Diptera253751; CNC_Diptera253754 (1ठ1우, TMAG); Franklin-Gordon Wild River National Park, Donaghy's Hill, -42.197778 145.931944, 11.i.2015; 7.i.1999, J.H., A.M. and A.W. Skevington; J. \& A. Skevington, M. Matheison, ovipositing in huge fallen log, CNC457454; CNC457499; CNC451893; UQIC_Reg94556; UQIC_Reg94777; UQIC_Reg94780; UQIC_Reg94782 (5ổ $\widehat{0}, 2$ 우, CNC, QM); Hobart, -42.882139 147.327194, 29.xii.1955; i.1984, G.H. Hardy, AustralianMuseumK407686;

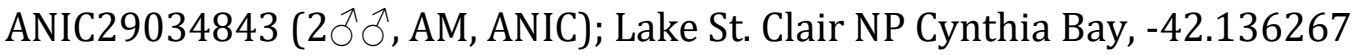
146.230931, 740m, 22.i.2006, M.P. van Zuijen; W. van Steenis, CNC_Diptera253782 (1ㅇ, CNC); Lake St. Clair NP Shadow Lake Circuit, -42.136267 146.230931, 800m; 850m, 22.i.2006, M.P. van Zuijen, CNC_Diptera253789-253790; CNC_Diptera253792 (3우우, WVSPC, MVZPC); Lake St. Clair Nat. Pk. 4 km N Derwent Bridge, -42.133333 146.183333, 29.i.1992, G. and A. Daniels, GDCBReg_19621, GDCBReg_19623 (10^, 1ㅇ, GDCB); Lake St. Clair National Park, Cynthia Bay, -42.125764 146.20603, 740m, 22.i.2006, M.P. van Zuijen; W. van Steenis, Jeff_Skevington_Specimen43816-43817; Jeff_Skevington_Specimen43823; Jeff_Skevington_Specimen43825 (4우, MVZPC, 
WVSPC); Launceston, -41.433197 147.144094, 28.x.1916, F.M. Littler, ANIC29034860 (1ð, ANIC); Lispootah Dam, -42.318258 146.472692, 14.xi.1975, K.R. Norris, ANIC29034522; ANIC29034552 (1ðð,1ㅇ, ANIC); Lyell Hwy: Franklin R nature trail, -42.215700 146.019800, on flowering Leptospermum, 11.i.2015, S.J. Grove, CNC_Diptera253819 (1ð̊, ANIC); Mole Creek, -41.558628 146.404517, 4.ii.1916, G.H. Hardy, ANIC29034531; ANIC2903457 (2우, ANIC); Mount William National Park; Summit of Mount William, -40.909722 148.186389, 23.xii.1998, J. \& A. Skevington, UQIC_Reg94786; UQIC_Reg94789; UQIC_Reg94793 (3ổ $\widehat{\jmath}$, UQIC); Mt Field NP Lake Belcher Track, -42.663558 146.649283, 1100m, 21.i.2006, M.P. van Zuijen; W. van Steenis, CNC_Diptera253793; Jeff_Skevington_Specimen43812; Jeff_Skevington_Specimen43814; Jeff_Skevington_Specimen43824; CNC_Diptera253791; CNC_Diptera253780; CNC661778; CNC661783 (3ðð,6우， WVSPC, MVZPC); Mt Field NP Tall Tree Nature Walk -42.663558 146.649283, 220m, 21.i.2006, W. van Steenis, CNC_Diptera254242 (19, WVSPC); Mt. Barrow Rd., 41.345000 147.352778, 12-14.xii.1998, D. Yeates, S. Winterton, malaise trap, QM_REG._NO._T221273-221275 (3우, UQIC); Mt. Barrow, -41.366667 147.416667, 1000m, 23.i.1992, G. and A. Daniels, GDCBReg_19619 (1q, UQIC); Mt. Wellington, Hobart, -42.916667 147.233333, 720m, 1.i.1992, G. Daniels, GDCBReg_1962719628, GDCBReg_19632; GDCBReg_19635-19636; GDCBReg_19638-19639

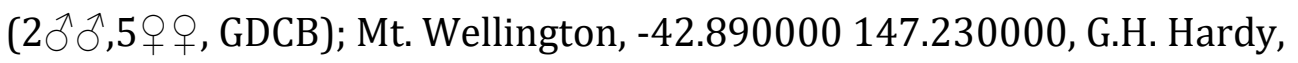
ANIC29034479 (1ㅇ, ANIC); North of Corinna, -41.588514 145.189764, 12.i.2015, J.H., A.M. and A.W. Skevington, CNC457434 (19, CNC); Peilon Hut, 3km S Mt. Oakleigh, -41.500000 146.030000, 5-10.ii.1990, I.D. Naumann, CNC_Diptera253697 
(1우, ANIC); Strahan, -42.150000 145.316667, ii.1924, G.H. Hardy, ANIC29034519; CNC_Diptera253850-253860 (12우, ANIC); Tarkine BushBlitz SG02 ridge NW of Lt Savage R, -41.472100 145.163200, 28.i.2015, S. Grove, CNC_Diptera253749 (1우, TMAG); Tarkine BushBlitz SG04 shrubland adj quarry at Browns Plain, -41.610000 145.175500, 29.i.2015, S. Grove, CNC_Diptera253757; CNC_Diptera253755; CNC_Diptera253748 $(2 \widehat{\jmath}, 1+$, TMAG); Warra Long Term Ecological Research Site 106, Grid: 744282, LTIS8211, -43.1 146.683333, 17.xi-16.xii.1998, malaise trap, UQIC_Reg94603 (1ㅇ, UQIC); Warra Long Term Research Site 59, Grid: 742279, LTIS8211, -43.1 146.683333, 14.i-12.ii.1998, malaise trap, UQIC_Reg94605 (1옹 UQIC); Victoria: 26 miles North Northeast, Orbost, -37.388111 148.591800, 396m, 6.xi.1969, I.F.B. Common, ANIC29034866 (10 ${ }^{\lambda}$ ANIC); Ararat, -37.284600 142.931600, H.W. Davey, ANIC29034830; ANIC29035021 (1§^,1ㅇ, ANIC); Bettersby's Camp, 15 km East of Nelson, lower Glenelg National Park, -38.030000 141.110000, 28.xi.1992, McAlpine, McEvey, Moulds, AustralianMuseumK407563 (1ㅇ, AM); Croydon, -37.796000 145.281000, 17.xi.1909, E.M. Fulton, ANIC29034853 (1ð^, ANIC); Grampians National Park, Reed's Lookout, -37.148111 142.448261, 23.x.2014, J.H., A.M. and A.W. Skevington, CNC385028-385030

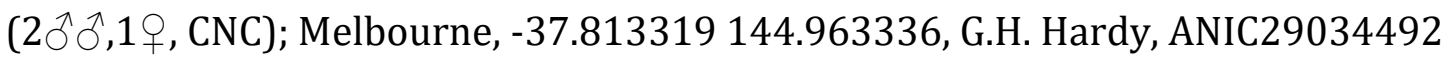
(1우, ANIC); Mt. Buffalo NP Dickson Falls , -36.731325 146.784042, 1440m, 29.i.2006, W. van Steenis, CNC_Diptera253781; CNC_Diptera253763, CNC_Diptera253776; CNC661775; Jeff_Skevington_Specimen43815;

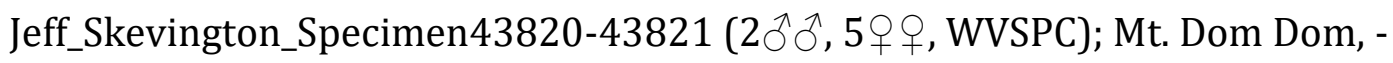
37.590278 145.657778, 22.x.1961, D.H. Colless, ANIC29034468 (19, ANIC); 
Princess Margaret, Rose Caves, 8 km North Northwest of Nelson, -37.59 140.58, 29.xi.1992, McAlpine, McEvey, Moulds, sweeping, AustralianMuseumK407565 (1ð̊, AM); Urquhart Bluff, -38.436336 144.130617, 19.xi.1975, K.R. Norris, ANIC29034879 (1ð, ANIC); Warburton District, -37.752539 145.694839, 9.xii.1900, ANIC29034539 (1q, ANIC); Wyperfeld National Park, Eastern Lookout, -35.594192 142.112061, 22.x.2014, J.H., A.M. and A.W. Skevington, CNC385032 (19, CNC); Wyperfield National Park, -35.588747 142.051722, 5.xi.1966, I.F.B. Common \& M.S. Upton, ANIC29035010 (1 $\overbrace{}^{\lambda}$ ANIC); Young's Ck 12 km N of Orbost Bohang Hwy., 37.609489 148.466761, 8.xi.1976, Colless \& Liepa, USNM_ENT249834;

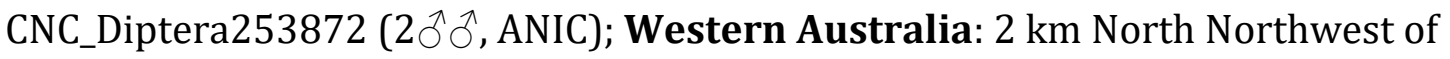
Crossman, -32.750000 116.566667, 24.ix.1981, I.D. Naumann, J.C. Cardale, ANIC29034834 (1 ${ }^{\lambda}$, ANIC); 24 mi. NW of Walpole, -34.681372 116.514911,

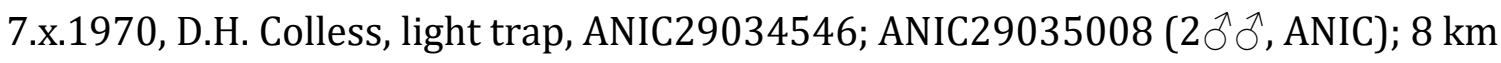
S Permberton, -34.483333 116.000000, 17.i.1987, G. and A. Daniels, GDCBReg_19730 (1우, GDCB); 9 km South Southwest of Bindoon, -31.448614 116.064672, 25.ix.1981, J.C. Cardale, light trap, ANIC29034996 (10̊, ANIC); Bluff Knoll Road, Stirling National Park, -34.399117 117.962769, 12.x.1970, D.H. Colless, ANIC29034924 (1ㅇ, ANIC); Circular Pool, -34.943903 116.799967, 9.x.1970, D.H. Colless, ANIC29034510 (19, ANIC); Cranmore Park, -30.602581 116.297339, 25.ix.1932, ANIC29034472; ANIC29034483 (2우우, ANIC); Crowea S/F Pemberton, 34.533467 116.156211, 15.xi.1978, S.J. Curry, ANIC29034524 (10^, ANIC); Crystal Springs, 7 miles West of Walpole, -34.903975 116.621292, on leaves of Xanthorrhoea, 14.xii.1970, G.A. Holloway \& H. Hughes, AustralianMuseumK408008 
(1ㅇ, AM); D'Entrecastaux Pk, -34.775028 116.163278, ex. coastal heath, flowering, 17.xi.2008, S.D. Gaimari \& S.L. Winterton, CNC661771; CNC661774 (1 §̊,1ㅇ, CSCA); Dwellingup, -33.000000 116.050000, 3.x.1981, I.D. Naumann, J.C. Cardale, ANIC29034494 (1 9 , ANIC); Hovea, -31.887233 116.115558, 29.ix.1935, K.R. Norris, ANIC29034447 (1ㅇ, ANIC); Leeuwin-Naturalists Natl Park, -34.050778 115.018167, ex. coastal heath, flowering, 16.xi.2008, S.D. Gaimari \& S.L. Winterton, CNC661773 (1へ̂, CSCA); Leewin Naturaliste NP: Boranup Beach Road, -34.159444 115.028889, 50m, coastal heath, 6.xi.2003, C. Lambkin, J. Recsei, A. Zwick, ANIC29035756 (1웅 ANIC); Mount Magog, Stirling Ranges, -34.399117 117.962769, 7-8.xii.1970, G.A. Holloway, malaise trap, AustralianMuseumK410145 (1ㅇ, AM); Mundaring, 31.897006 116.171136, 23.viii.1926, E.W. Ferguson, ANIC29034448; ANIC29034992 (1§,1우, ANIC); Nannup, -33.982000 115.765000, 12.xi.1958, E.F. Riek, ANIC29034544 (1ðð, ANIC); Pemberton, -34.440000 116.050000, 29.xi.1936, K.R. Norris, ANIC29034874 (1 $\overbrace{}^{\AA}$, ANIC); Porongorup National Park, Summit of Morgan's View, -34.680161 117.868214, hilltop, 25.i.2015, J.H., A.M. and A.W. Skevington, CNC451880 (1ð^, CNC); S.W. District, ix.1938, E.H. Kipps, ANIC29034477 (1ㅇ, ANIC); Stirling NP n. side of range , -34.399117 117.962769, ex: Eucalyptus, 25.xii.1994, LS \& RB Kimsey, CNC_Diptera255000 (1ㅇ, CNC); Stirling Nature Park, 49 mile post, -34.399117 117.962769, 12.x.1970, D.H. Colless, ANIC29034503; CNC_Diptera253849 (2우, ANIC); Stirling Nature Park, 54 mile post, -34.399117 117.962769, 12.x.1970, D.H. Colless, ANIC29034502 (1우, ANIC); Stirling Range Drive, Stirling Range National Park, -34.399117 117.962769, 23.ix.1981, I.D. Naumann, J.C. Cardale, ANIC29034995 (10^, ANIC); Stirling Range National Park, 
Bluff Knoll, Hilltop, -34.377500 118.252222, 28.xii.1999, J.\&A. Skevington, QM_REG._NO._T221265 (1ð, UQIC); Stirling Range, Mount Abbey, -34.349992 117.950172, 20.x.1977, Signe Holm leg., JeffSkevingtonSpecimen\#31419 (1ð, ZMUC); Stirling Range, Mount Hassel, -34.377776 118.068697, 25.x.1977, Signe Holm leg., JeffSkevingtonSpecimen\#31420 (10^, ZMUC); Stirling Range, Toolbrunup, -34.382569 118.049252, 20.x.1977, Signe Holm leg., JeffSkevingtonSpecimen\#31418 (1ð, ZMUC); Summit of Mount Barker, -34.655692 117.647083, hilltop, 26.i.2015, J.H., A.M. and A.W. Skevington, CNC452068-452069

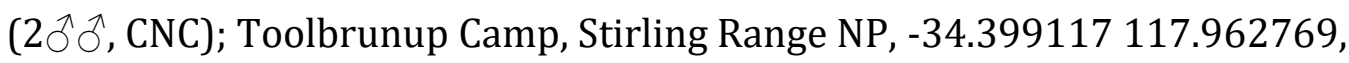
22.ix.1981, J.C. Cardale, ANIC29034525 (1ð ${ }^{\lambda}$ ANIC); Treen Brook, 5 miles West of Pemberton, -34.449886 115.950942, 19.xii.1970, G. Williams \& H. Hughes, Malaise trap, AustralianMuseumK410505 (1, AM); Warren River, 6 miles Southeast of Pemberton, -34.511628 116.114703, 17.i.1971, G.A. Holloway \& H. Hughes, AustralianMuseumK410130 (10, AM); near Warren River, $10 \mathrm{~km}$ South of Pemberton, -34.534142 116.046947, 27-29.ix.1987, D.K. McAlpine, AustralianMuseumK407956 (1, AM).

\section{Psilota viridis Macquart, 1847}

(Species plate: Fig. 34, Genitalia: Fig. 42E+F, Map: 29)

Psilota viridis Macquart, 1847: 62. Type locality “Nouvelle-Hollande”, Australia.

Body length: 7.8-12.5 mm. 
Diagnosis: Metallic blue-green species with face dark, frons entirely pale pilose or with some dark pili, pleuron shining and partly white pilose. Hind femur with a distinct rounded apicoventral setulose carina. Hind tibia simple. The dark face distinguishes $P$. viridis from $P$. pallidifacies, while the blue-green colouration and white peural pilosity distinguishes it from $P$. coerulea.

Description: very similar to Psilota coerulea, differing as follows:

Head: Pollinosity and pilosity of face white or yellow-white. Pilosity of frons either entirely white or with some dark pili mixed in. Antenna usually brown on the dorsal half of the scape, pedicel, and postpedicel.

Thorax: Ground colour lighter, metallic green-blue. Legs somewhat more orange in most specimens. Apical half of fore- and mid femora often with an anterior orange streak, all tibiae and tarsomeres often partly to entirely orange-brown. Pile on pleuron almost entirely white, sometimes with individual black pili on the posterior anepisternum, anepimeron, and/or the ventral half of the katepisternum. Halter pale orange. Calypter pale, with a dark brown border.

Genitalia: Epandrium compact, about as long as tall. Cercus approximately triangular. Outer lobe of surstylus inserted at mid-height of inner lobe, curved 
downwards on apical $1 / 3^{\text {rd }}$, with apex of lobe thickened and curved backwards towards base of surstylus. Inner lobe of surstylus ventrally swollen at base, otherwise broadening gradually over entire length, rounded at apex. Phallapodeme smooth. Gonostylus slightly darkened.

Discussion: The syntype series for Psilota viridis is a mixed series of females of $P$. viridis and P. coerulea. The lone specimen from the Oxford Museum of Natural History is a female $P$. viridis labelled "Holotype", but as Macquart did not designate a holotype, this is likely part of a syntype series. The remainder of the series is stored in the Paris Natural History Museum, and these appear to be female P. coerulea. Since viridis is a name that is currently in use in entomological collections, we have chosen to nominate the Oxford Museum (OUMNH) specimen as a lectotype and thus retain the current concept for this species, rather than subsuming the name within P. coerulea. Therefore, the female specimen (Jeff_Skevington_Specimen45697) from the Oxford museum is here designated as the lectotype in order to stabilize the concept of this species.

Additionally, there may be two species under the name Psilota viridis, as frons pilosity colouration rarely varies within a species. But, because range, genitalia, and COI barcodes are identical between pale pilose and dark pilose specimens, we refer to this as a single species for now. 
Specimens Examined: LECTOTYPE Material: AUSTRALIA: Female P. viridis, with labels reading "Holotype”, “J. Skevington Specimen \#45697”, “P. viridis EX COLL. BIGOT" (1 $q$ OUMNH). PARALECTOTYPE Material: AUSTRALIA: Five female $P$. coerulea, with labels reading "Syntype”; "13 44”; “MNHN, Paris ED9369”, MNHN, Paris ED9370”, “MNHN, Paris ED9371”, “MNHN, Paris ED9372”, MNHN, Paris ED9373". (5우, MNHN, photographs). Other specimens: AUSTRALIA. Australian Capital Territory: Black Mountain, -35.160000 149.060000, 1.i.1994, D.K. Yeates, UQIC_Reg94730 (1§ึ, UQIC); Back Mountain, -35.273675 149.097583, i.1988, M.E. Irwin, Malaise trap, ANIC29035771 (10 ANIC); Canberra, -35.282000 149.128686, 1-10.xii.1953; 1-15.xi.1953; 15.i.1934; 20.xi.1958; 23.xi.1947; 7.xi.1948, F. M. Hull; I.M. Mackerras; Z. Liepa; Paramonov, CNC_Diptera254984-254985;

CNC_Diptera255058; CNC_Diptera255003-255008; CNC_Diptera255010-255012; CNC_Diptera255016; CNC_Diptera255029; CNC_Diptera255033-255037; ANIC29034906-29034907; ANIC29035018; CNC_Diptera255026;

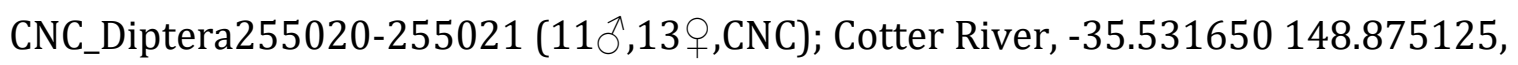
on Leptospermum, 23.xi.1929, L.M. Williams, ANIC29034454 (19, ANIC); New South Wales: 99 km North of Wentworth, -33.027339 150.952214, Hakea leucoptera, 30.xi.1992, N.W. Rodd, AustralianMuseumK410151 (1우, AM); Biniguy, -29.489017 150.115050, 17.ix.1923, ANIC29034914 (1ð^, ANIC); Bogan River, -30.478233 146.714608, ANIC29034461; ANIC29034919 (2우, ANIC); Canyonlea [Canyonleigh] Road near Mossvale, -34.535442 150.137775, 12.ix.1966, M.L. Dyce, with pupal case (emerged 12.X.66) [larva in spirit], ANIC29034917 (1へ , ANIC); Clifton Downs - Mt. Wood, -29.354242 142.170308, 1.xi.1949, S.J. Paramonov, 
USNM_ENT249835 (1ð^, USNM); Como near Sydney, -33.926375 151.125936, 1992, A. Musgrave, Date is ambiguous reads "1.3.1924", K49224, AustralianMuseumK408023 (1ㅇ, AM); Durras Bay, -35.645003 150.291439,

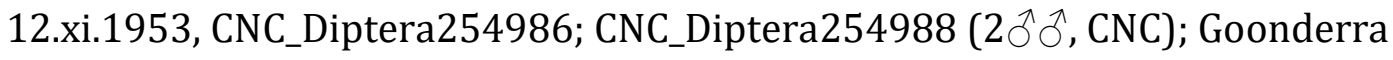
Ridge, Royal National Park, -34.114342 151.046586, 4.x.1969, G. Daniels, GDCBReg_19712 (1ㅇ, GDCB); Hazelbrook, -33.733375 150.449989, ANIC29034465;

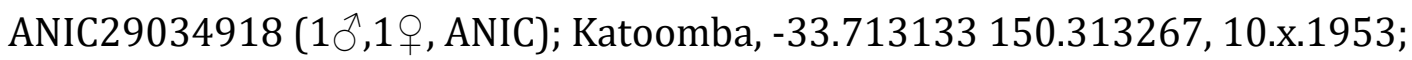
10.x.1958, F.M. Hull, CNC_Diptera255022; CNC_Diptera255030; CNC_Diptera255032; CNC_Diptera255039; CNC_Diptera255009 (3ðð 2 2 $+\uparrow, C N C)$; Mooney Mooney Ck nr Gosford , -33.437894 151.246467, 3.xii.1976, G. Daniels; D.K. McAlpine, B.J. Day, D. Jenkins, GDCBReg_19770; AustralianMuseumK408028; AustralianMuseumK408030 (2ðð1우, GDCB, AM); Nullo Mountain 20 miles Northeast of Rylstone, -32.737947 150.226400, 12.xi.1950, T.G. Campbell, ANIC29034803 (1ㅇ, ANIC); Orange, -33.284228 149.102683, 25.xii.1951, Z. Liepa, ANIC29034813 (1q, ANIC); Sydney, -33.926375 151.125936, 18.x.1925, Health Dept., ANIC29034921 (19, ANIC); xi.1924, ANIC29034920 (1, ANIC); Tibooburra, Cobham Lake, -30.146858 142.087394, 17.xi.1949, S.J. Paramonov, USNM_ENT249836 (1ðึ, USNM); Tregeagle, 10km SE Lismore, -28.857939 153.350561, 25.viii.1984, D.K. Yeates, UQIC_Reg94659 (1ð^, UQIC); Warrumbungle NP, Brownes Ck., -31.170000 148.580000, 13-15.i.1994, M.E. Irwin, D.K. Yeates, Malaise trap, UQIC_Reg94679 (1ð̂, UQIC); near Bourke, -30.090661 145.938194, 26.x.1949, S.J. Paramonov, ANIC29034905; CNC_Diptera255082;

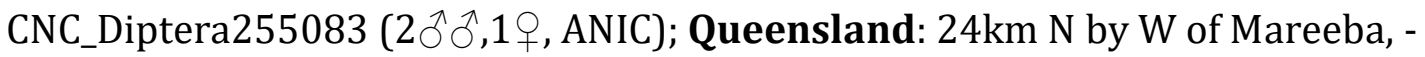


16.470000 145.220000, 25.xi.1981, D.H. Colless, ANIC29034462 (1우, ANIC); 5 km N Leyburn, -27.966667 151.633333, 22.x.1986, G. and A. Daniels, GDCBReg_12258 (1우, GDCB); 5km W. Toobeah, -28.425342 149.804014, on Atalaya hemiglauca, 16.xi.1979, K.L. Walker, UQIC_Reg94591 (1우, UQIC); Amiens State Forest nr Stanthorpe, -28.600522 151.799608, 24-26.xi.1981; 27.xii.1987, G. Daniels and M.A. Schneider; G. and A. Daniels, UQIC_Reg94574; GDCBReg19514 (2ðð Barakula SF, site 12, -26.427000 150.506000, 249m ;406m, 23.i-10.ii.2010; 822.i.2010, Monteith, Turco, Malaise trap, QM_REG._NO._T222169-222170 (2우오, QM); Bribie Island, -26.986100 153.132525, 6.ix.1955, ANIC29035765 (1ㅇ, ANIC); Brisbane, -27.526975 153.040322, 10.ix.1939; 12.ix.1916; 15.ix.1915; 1941; 26.ix.1916; 7.ix.1927; ix.1935, C.F. Ashby; H. Hacker; J. Mann; G.H. Hardy, CNC_Diptera255025; QM_REG._NO._T223188-223189; ANIC29034922; QM_REG._NO._T223193; ANIC29034915; QM_REG._NO._T223195; QM_REG._NO._T223192; UQIC_Reg94806; UQIC_Reg94819;

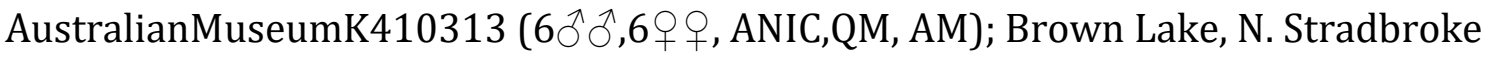
Is., S.E.Q., -27.490108 153.432378, on Leptospermum flavescens blossom, 2124.ix.1984, D.K. Yeates, UQIC_Reg94657 (1우, UQIC); Bundaberg, -24.866972 152.350972, ix.1971; viii-ix.1971, H. Frauca, CNC_Diptera255084-255087;

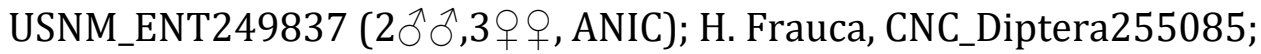
CNC_Diptera255086; CNC_Diptera255087 (ANIC); Caboolture, -28.083333

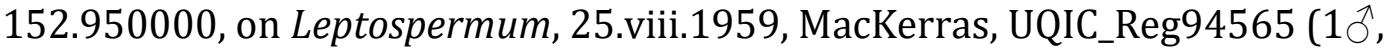
UQIC); Carlisle Island, -20.788936 149.285100, 9-17.xii.1986, S.R. Monteith, QM_REG._NO._T223225 (1ㅇ, QM); Carnarvon Nat. Park, Mt. Moffatt Section, 3km SE 
Ranger Station, -25.077500 148.008333, 740m, 18.xi.1995, D.K. Yeates, UQIC_Reg94799 (1§^, UQIC); Cleveland, -27.533333 153.266667, viii.1924, G.H. Hardy, ANIC29034455; ANIC29034459 (2ㅇ, ANIC); Collinsville, -20.550147 147.850028, 15.ix.1950, E.F. Riek, ANIC29034931 (1+, ANIC); Culgoa Floodplain NP, Reedy Swamp, 7km NW Toulby Gate, -28.955000 146.875000, 138m, 18.xi.2009, C. Lambkin, N. Starick, QM_REG._NO._T222167-222168 (1ð^1ㅇ, QM); Dayboro, 0.5 km South, -27.200000 152.816667, 18-19.ix.1999, S.G. Evans, QM_REG._NO._T223221223223 (3우우, QM); Durham Downs- Nokandra, -26.116933 149.076992, 13.xi.1949, S.J. Paramonov, ANIC29034927 (1ㅇ, ANIC); Lake Broadwater nr Dalby, 27.350000 151.100000, 26.x.1986, G. and A. Daniels, GDCBReg19515 (10^, GDCB); Mahogany Forest, Mount Moffat National Park, -24.933333 148.066667, 1200m, 2426.ii.1996, C.J. Burwell, S. Evans, light trap, QM_REG._NO._T223224 (19, QM); McPherson Range, -28.333333 153.000000, 12.xi.1949, S.J. Paramonov,

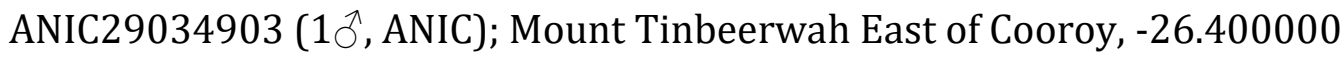
152.983333, hilltopping, 7.xi.1998; 29.ix.1985, G. and A. Daniels, UQIC_Reg94776; GDCBReg_19557 (1ð^,1ㅇ, GDCB, UQIC); Mount Walsh summit, -25.583333 152.050000, 640m, open forest \& rock slabs, 2-3.xii.1998, C.J. Burwell, S.G. Evans, QM_REG._NO._T223194; QM_REG._NO._T223226 (2ððð, QM); Mt Marlay near Stanthorpe, S.E.Q., -28.653583 151.946908, 24-25.xi.1984, D.K. Yeates, U.Q.I.C. Loan \#1036, UQIC_Reg94658 (1ðึ, UQIC); N. Bundaberg, -24.866972 152.350972, in wallum, ix.1972, H. Frauca, CNC_Diptera255028 (1ð̊, ANIC); Orientos Nappamerrie, -27.600878 141.106858, 5.xi.1949, S.J. Paramonov, ANIC29034902

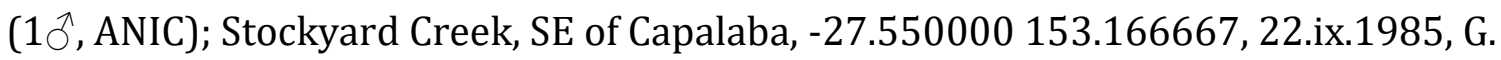


and A. Daniels, GDCBReg_19562 (1우, UQIC); The Beacon, Imbil State Forest, 26.500000 152.583333, 29.xii.1990, G. and A. Daniels, GDCBReg_19518 (1우, GDCB); The Blunder Brisbane, -27.526975 153.040322, 18.ix.1966; 2.x.1966; 26-29.ix.1966; 4.x.1968, C.F. Ashby, CNC_Diptera255088-255099 (3ðð,8우우, ANIC); Tibrogargan Ck. , -26.933333 152.950000, Leptospermum flavescens, 30.viii.1957, F.A. Perkins, sweeping, UQIC_Reg94598 (19, UQIC); Tinnanbar, 4km SW, -25.783333 152.916667, 10m, sweeping Leptospermum, 10-12.x.2002, Burwell, Wright \& Cook, QM_REG._NO._T222140 (19, QM); Watten, -21.009561 144.062050, M. Taylor, ANIC29034460 (1ㅇ, ANIC); Yeppoon, -23.133408 150.733383, 20.ix.1954, I.F.B. Common, ANIC29034930 (1ㅇ, ANIC); Tasmania: Lime Bay Nature Reserve near Saltwater, -42.972222 147.707222, 3.i.1999, J. Skevington, UQIC_Reg94555 (10ิ่, UQIC); Victoria: Tarra Bulga N, Tarra Bulga Vis. Centre, -38.429742 146.566047, 27.i.2006, W. van Steenis, AMG 55 462-5746, CNC_Diptera255045 (1へ̂, CNC).

\section{Psilota zophos Young sp. nov.}

(Species plate: Fig. 35, Genitalia: Fig. 42G+H, Map: 30)

Body length: 7.9-13.6 mm.

Diagnosis: Face black, densely grey pollinose, with white pile. Thorax black. Hind femur with a distinct rounded apicoventral setulose carina. Hind tibia with basoventral spur. Calypter with dark brown border. Halter orange- 
brown to dark brown. Abdomen black, with faint metallic blue/green sheen. Outer lobe of surstylus only slightly curved back towards base of surstylus at apex, distinguishing it from $P$. femoralis.

Description: Very similar to $P$. femoralis, differing as follows:

Thorax: halter orange-brown to dark brown.

Abdomen: Abdomen black, with faint metallic blue/green sheen.

Genitalia: Epandrium compact, about as long as tall. Cercus approximately triangular. Outer lobe of surstylus inserted just below mid-height of inner lobe, curved downwards on basal $1 / 3^{\text {rd }}$, with apex of lobe thickened and slightly curved backwards towards base of surstylus. Inner lobe of surstylus more strongly broadened over entire length, with apex rounded.

Discussion: Nothing is known about the biology of this species.

Etymology: zophos is derived from Greek, meaning "darkness, or gloom". This is in reference to the gloomy black colouration of Psilota zophos. 


\section{Specimens Examined: HOLOTYPE material: AUSTRALIA. Western Australia:}

Cape Le Grand Nat Park; -33.966667 122.116667; 11.I.1987; G. and A. Daniels, GDCBReg_19526, (1 $\overbrace{}^{\Uparrow}$ GDCB). Paratypes: AUSTRALIA. Western Australia: 3 km N of Hopetoun, -33.916667 120.133333, 13.i.1987, G. and A. Daniels, GDCBReg_19728 (10ิ, GDCB); 5 mi. E. of Nornalup, -35.004314 116.880339, 8.x.1970, D.H. Colless, USNM_ENT_00249825 (1ðð, USNM); same data as holotype, GDCBReg_19525; GDCBReg19523 (2우, GDCB); Cape Le Grand Nat. Park, -33.966667 122.116667, hilltop, 10.i.1987; 12.i.1987, G. and A. Daniels; C.J. Burwell, GDCBReg_19521 (2ðððે, GDCB, UQIC).

\section{Species not recognized}

Psilota erythrogaster Curran, 1926: 120 is a species with a red abdomen known from the holotype female (type locality: Australia). It is a possible synonym of $P$. cupreus, but the species complex requires further revisionary work. See P. cupreus discussion above.

Psilota hirta Klocker, 1924: 54 is an all-black species known from the holotype female (type locality: Brisbane) and series of specimens determined by the original author. It is a possible synonym of $P$. tristis, but the species complex requires further revisionary work. See P. tristis discussion above. 
Psilota queenslandica (Klocker, 1924: 55) is an all-black species known from the holotype male (type locality: Brisbane) and series of specimens determined by the original author. It is a possible synonym of $P$. tristis, but the species complex requires further revisionary work. See $P$. tristis discussion above.

Psilota rubra Klocker, 1924: 53 is a species with a red abdomen known from the holotype female (type locality: Brisbane) and series of specimens determined by the original author. It is a possible synonym of $P$. cupreus, but the species complex requires further revisionary work. See Psilota cupreus discussion above.

Psilota rubriventris (Bigot, 1885: 248) is a species with a red abdomen known from the holotype female (type locality: Australia). It is a possible synonym of $P$. cupreus, but the species complex requires further revisionary work. See P. cupreus discussion above.

Psilota shannoni Goot, 1964: 220 is a replacement name assigned to Psilota nigra, 1927. It is an all-black species known from the holotype female (type locality: South Australia). It is a possible synonym of $P$. tristis, but the species complex requires further revisionary work. See $P$. tristis discussion above. 
Psilota victoria Curran, 1925: 7 is a replacement name for the pre-occupied Psilota cyanea Hill, 1921. This North Queensland species was collected in association with termites in Townsville. Unfortunately, all that remains of the type specimen is a bare pin, and other specimens mentioned by the author are apparently lost. From the original description, it is evident that $P$. victoria is one of the species similar to $P$. coerulea, P. darwini, etc., with a metallic green body and a lobe-shaped carina on the hind femur. The thoracic pile is recorded as being black, which fits the description of P. coerulea, but Townsville is well beyond the northernmost extent of $P$. coerulea's recorded range. Similarly, P. darwini fits the recorded range, but has a white pilose thorax. 


\section{References}

Bigot, J. (1885). "Diptères nouveaux ou peu connus. 28e. partie. XXXVI. Syrphidi. Addenda au mémoire publié dans les Annales de la Société Entomologique de France (Années 1883-1884)." Ann. Soc. ent. France 6(5): 24-252.

Birtele, D. and S. Hardersen (2012). "Analysis of vertical stratification of Syrphidae (Diptera) in an oak-hornbeam forest in northern Italy." Ecological research 27(4): 755-763.

Cumming, J. M. and D. M. Wood (2017). Adult morphology and terminology. In KirkSpriggs, A. H. and Sinclair, B. I., eds, Manual of Afrotropical Diptera. Volume 1. Introductory chapters and keys to Diptera families. Suricata 4. South African National Biodiversity Institute, Pretoria.

Curran, C. H. (1925). "New Diptera in the American Museum of Natural History." American Museum Novitates 176: 1-10.

Curran, C. H. and E. Bryan Jr (1926). New Australian Syrphidae (Diptera) in the Bishop Museum. Proceeding of the Linnean Society of New South Wales. Evenhuis, N. L. (2018). "Abbreviations for the insect and spider collections of the world." Retrieved Feb 28, 2018, from http://hbs.bishopmuseum.org/codens/.

Glumac, S. (1960). "Phylogenetical system of the syrphid-flies (Syrphidae Diptera) based upon the male genitalia structure and the type of the larvae with characteristics of the family and tribes." Glasnik Prirodnjackog muzeja Beogradu (B) 82: 82-103. 
Hajibabaei, M., J. R. deWaard, N. V. Ivanova, S. Ratnasingham, R. T. Dooh, S. L. Kirk, P. M. Mackie and P. D. N. Hebert (2005). "Critical factors for assembling a high volume of DNA barcodes." Philosophical Transactions of the Royal Society B: Biological Sciences 360(1462): 1959-1967.

Hill, G. (1921). Notes on some Diptera found in association with termites. Proceedings of the Linnean Society of New South Wales.

Kassebeer, C., A. Maibach and G. Rotheray (1998). "The third (= final) stage larva of Psilota anthracina Meigen and Psilota decessa (Hutton)(Dipt., Syrphidae)." Entomologist's Monthly Magazine 134(4-7): 39-43.

Klocker, C. (1924). "On a collection of Syrphidae from Queensland with descriptions of a new genus and eight new species: Queensland Mus." Brisbane), Mem 8: $53-60$.

Littlefield, R. (2018). Zerene Stacker.

Macquart, J. (1846). "Dipteres exotiques nouveaux ou peu connus. Supplément." Mémoires de la Société (Royale) des Sciences, de l'Agriculture et des Arts á Lille 1844: 133-364.

Macquart, J. (1847). Dipteres exotiques nouveaux ou peu connus. 2.e supplement. Paris, Roret.

Macquart, J. (1851). "Dipteres exotiques nouveaux ou peu connus. Suite du 4e supplement publie dans les Memoires de 1849." Mémoires de la Société Royale des Sciences, de l'Agriculture et des Arts de Lille 1850: 134-294.

Maddison, W. P. and D. R. Maddison (2010). Mesquite: a modular system for evolutionary analysis. 
Meijere, J. d. (1933). "Über Dipteren, deren Larven in Termitennestern leben, nebst über einige weitere, z. T. neue, gleichfalls aus Djati-Wäldern auf Java." Tijdschrift voor Entomologie 76: 103-144.

Miller, D. (1921). "Material for a Monograph on the Diptera Fauna of New Zealand: Part II, Family Syrphidae." Transactions of the New Zealand Institute 53: 289-333.

Peck, L. V. (1988). Family Syrphidae. Catalogue of Palaearctic Diptera. Volume 8. Syrphidae-Conopidae. A. Soos and L. Papp. Budapest, Hungary, Akadémiai Kiadó. 8: 11-230.

Rotheray, G. and F. Gilbert (1999). "Phylogeny of Palaearctic Syrphidae (Diptera): evidence from larval stages." Zool 」 Linn Soc 127: 1-112.

Schiner, J. R. (1868). Diptera. Reise der osterreichischen Fregatte Novara, Zool. B. v. Wüllersdorf-Urbair. Wien. 2: 1-388.

Sequencer. (2018). Sequencher DNA sequence analysis software. Ann Arbor, MI, USA, Gene Codes Corporation.

Shannon, R. (1927). "A new genus and five new species of Syrphidae from Australia (Diptera)." Proceedings of the Entomological Society of Washington 29(4): 82-87.

Shatalkin, A. I. (1975). "A taxonomic analysis of the Hover Flies (Diptera, Syrphidae). I." Entomological Review 54(1): 117-125.

Shorthouse, D. P. (2010). "SimpleMappr, an online tool to produce publicationquality point maps." 2 April 2012. Retrieved 20 April 2012, 2012, from http://www.simplemappr.net. 
Smit, J. T. V., A. (2007). "The Palaearctic species of the genus Psilota Meigen (Diptera, Syrphidae) with the description of two new species." Studia dipterologica 24(2): 345-364.

Speight, M. (2016). Species accounts of European Syrphidae (Diptera), Glasgow 2016. Syrph The Net, the database of European Syrphidae. Vol. 93, Syrph The Net publications, Dublin.

Thompson, F. C. (1972). "A contribution to a generic revision of the Neotropical Milesinae (Diptera: Syrphidae)." Arquivos de Zoologica 23(2): 73-215.

Thompson, F. C. (1999). "A key to the genera of the flower flies (Diptera: Syrphidae) of the Neotropical Region including descriptions of new genera and species and a glossary of taxonomic terms." Contributions on Entomology, International 3(3): 322-378.

Van der Goot, V. (1964). "Fluke's catalogue of Neotropical Syrphidae (Insecta, Diptera), a critical study with an appendix on new names in Syrphidae." Beaufortia 10(127): 212-221.

Walker, F. (1852). Diptera [cont.]. Insecta Saundersiana [q.v.]. W. W. Saunders. London. 1: 216.

Walker, F. (1858). "Catalogue of the dipterous insects collected in the Aru Islands by Mr. AR Wallace, with descriptions of new species." Zoological Journal of the Linnean Society 3(10): 77-110.

Wirth, W. W., Y. S. Sedman and H. V. J. Weems (1965). Family Syrphidae. A catalog of the Diptera of America north of Mexico. A. Stone, C. W. Sabrosky, W. W. Wirth, 
R. H. Foote and J. R. Coulson. Washington, United States Department of Agriculture. Agriculture Handbook no. 276: 557-625. 
Chapter 3, Table 1: World Psilota names in literature record. Species marked with a * are junior synonyms, species marked with ${ }^{* *}$ have been assigned to different genera, and specimens marked with $\mathrm{a}^{\dagger}$ are junior homonyms. $\mathrm{IM}=$ Indomalayan, $\mathrm{NE}=$ Nearctic, $\mathrm{AU}=$ Australian, PA = Palaearctic.

\begin{tabular}{|c|c|c|c|c|c|c|}
\hline original genus & original species & valid genus & valid species & author & year & biotic range \\
\hline Psilota & cyanea** & Heringia & cyanea & Brunetti & 1915 & IM \\
\hline Psilota & bifenestrata & Psilota & bifenestrata & Meijere & 1933 & IM \\
\hline Psilota & fasciata & Psilota & fasciata & Curran & 1929 & IM \\
\hline Psilota & tectonae & Psilota & tectonae & Meijere & 1933 & IM \\
\hline Psilota & shewelli & Psilota & shewelli & Thompson & 2012 & IM \\
\hline Pipiza & buccata & Psilota & buccata & Macquart & 1842 & $\mathrm{NE}$ \\
\hline Psilota & flavidipennis & Psilota & flavidipennis & Macquart & 1855 & $\mathrm{NE}$ \\
\hline Psilota & thatuna & Psilota & thatuna & Shannon & 1922 & $\mathrm{NE}$ \\
\hline Chrysogaster & cupreus & Psilota & cupreus & Macquart & 1850 & AU (Tas) \\
\hline Brachyopa & rubiventris & Psilota & rubiventris & Bigot & 1885 & $\mathrm{AU}$ \\
\hline Orthonevra & basalis & Psilota & basalis & Walker & 1858 & $\mathrm{AU}$ \\
\hline Melanostoma & decessum & Psilota & decessum & Hutton & 1901 & $\mathrm{AU}(\mathrm{NZ})$ \\
\hline Coiloprosopa & nitida & Psilota & nitida & Macquart & 1850 & $\mathrm{AU}$ \\
\hline Emmyia & queenslandica & Psilota & queenslandica & Klocker & 1924 & $\mathrm{AU}$ \\
\hline Merodon & muscaeformis* & Psilota & coerulea & Walker & 1852 & $\mathrm{AU}$ \\
\hline Psilota & auricauda & Psilota & auricauda & Curran & 1926 & $\mathrm{AU}$ \\
\hline Psilota & coerulea & Psilota & coerulea & Macquart & 1846 & $\mathrm{AU}$ \\
\hline Psilota & erythrogaster & Psilota & erythrogaster & Curran & 1926 & $\mathrm{AU}$ \\
\hline Psilota & femoralis & Psilota & femoralis & Schiner & 1868 & $\mathrm{AU}$ \\
\hline Psilota & hirta & Psilota & hirta & Klocker & 1924 & $\mathrm{AU}$ \\
\hline Psilota & rubra & Psilota & rubra & Klocker & 1924 & $\mathrm{AU}$ \\
\hline Psilota & shannoni & Psilota & shannoni & Goot & 1964 & $\mathrm{AU}$ \\
\hline Psilota & nigra $^{\dagger}$ & Psilota & shannoni & Shannon & 1927 & $\mathrm{AU}$ \\
\hline Psilota & tristis & Psilota & tristis & Klocker & 1924 & $\mathrm{AU}$ \\
\hline Psilota & cyanea $^{\dagger}$ & Psilota & victoria & Hill & 1921 & $\mathrm{AU}$ \\
\hline Psilota & victoria & Psilota & victoria & Curran & 1925 & $\mathrm{AU}$ \\
\hline Psilota & viridis & Psilota & viridis & Macquart & 1847 & $\mathrm{AU}$ \\
\hline Psilota & nigra* & Chrysosyrphus & nasuta & Zetterstedt & 1843 & PA \\
\hline Psilota & toubkalana* & Psilota & atra & Kassebeer & 1995 & PA \\
\hline Pipiza & atra & Psilota & atra & Fallen & 1817 & PA \\
\hline Pipiza & curvinervis* & Psilota & innupta & Strobl & 1898 & $\mathrm{PA}$ \\
\hline Psilota & innupta & Psilota & innupta & Rondani & 1857 & $\mathrm{PA}$ \\
\hline Psilota & anthracina & Psilota & anthracina & Meigen & 1822 & PA \\
\hline Psilota & conjugata* & Psilota & anthracina & Rondani & 1857 & PA \\
\hline Psilota & rotundicornis* & Pipiza & quadrimaculata & Strobl & 1898 & PA \\
\hline Psilota & brevicornis & Psilota & brevicornis & Shiraki & 1968 & $\mathrm{PA}$ \\
\hline Psilota & nigripilosa & Psilota & nigripilosa & Shiraki & 1968 & PA \\
\hline Psilota & sibirica* & Psilota & innupta & Violovitsch & 1980 & PA \\
\hline Psilota & dersu* & Psilota & brevicornis & Violovitsch & 1980 & PA \\
\hline Psilota & plumbella* & Psilota & innupta & Becker & 1907 & $\mathrm{PA}$ \\
\hline Psilota & ruficornis $* *$ & Lejota & ruficornis & Wahlberg & 1843 & PA \\
\hline Psilota & kroska & Psilota & kroska & Mutin & 1999 & PA \\
\hline Psilota & nana & Psilota & nana & Smit and Vujic & 2008 & PA \\
\hline Psilota & exilistyla & Psilota & exilistyla & Smit and Vujic & 2008 & $\mathrm{PA}$ \\
\hline
\end{tabular}




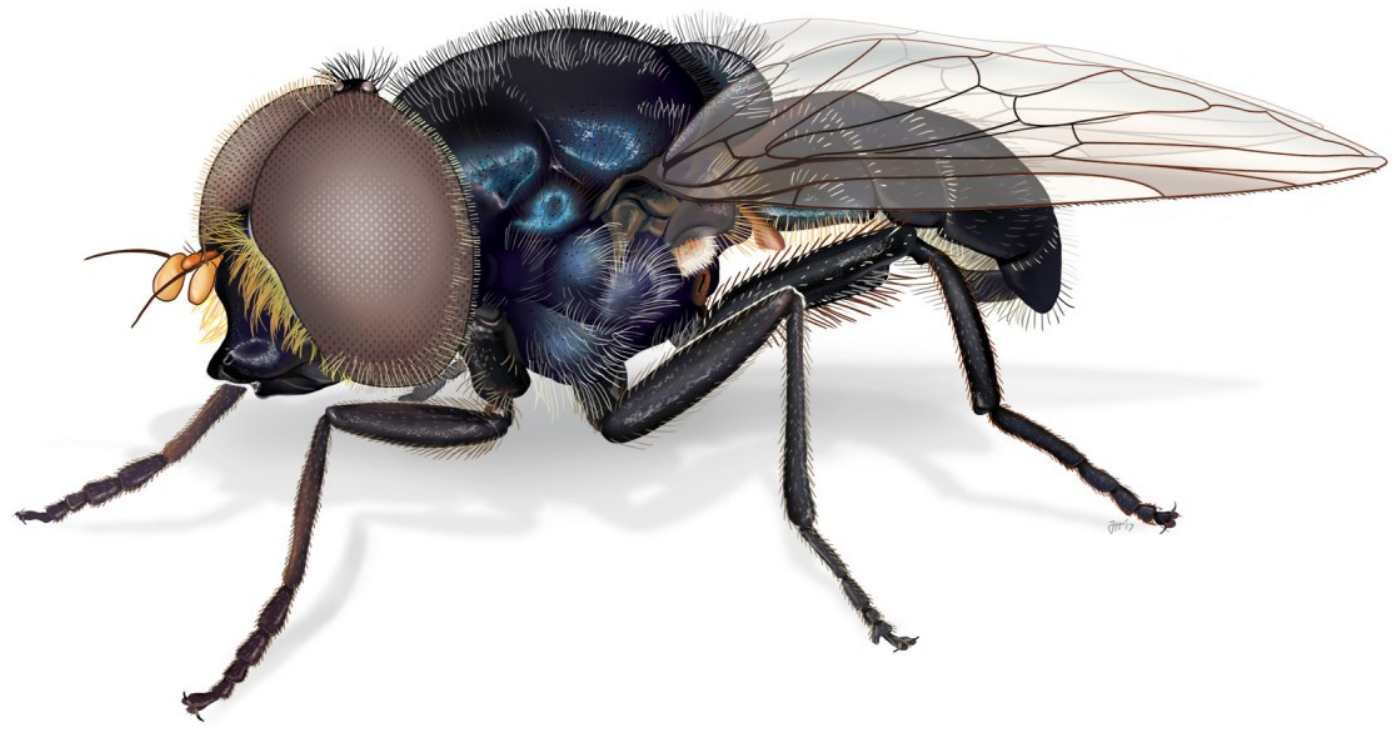

Chapter 3, Figure 1. Psilota alexanderi, general habitus. Illustration by Jessica Hsiung. 


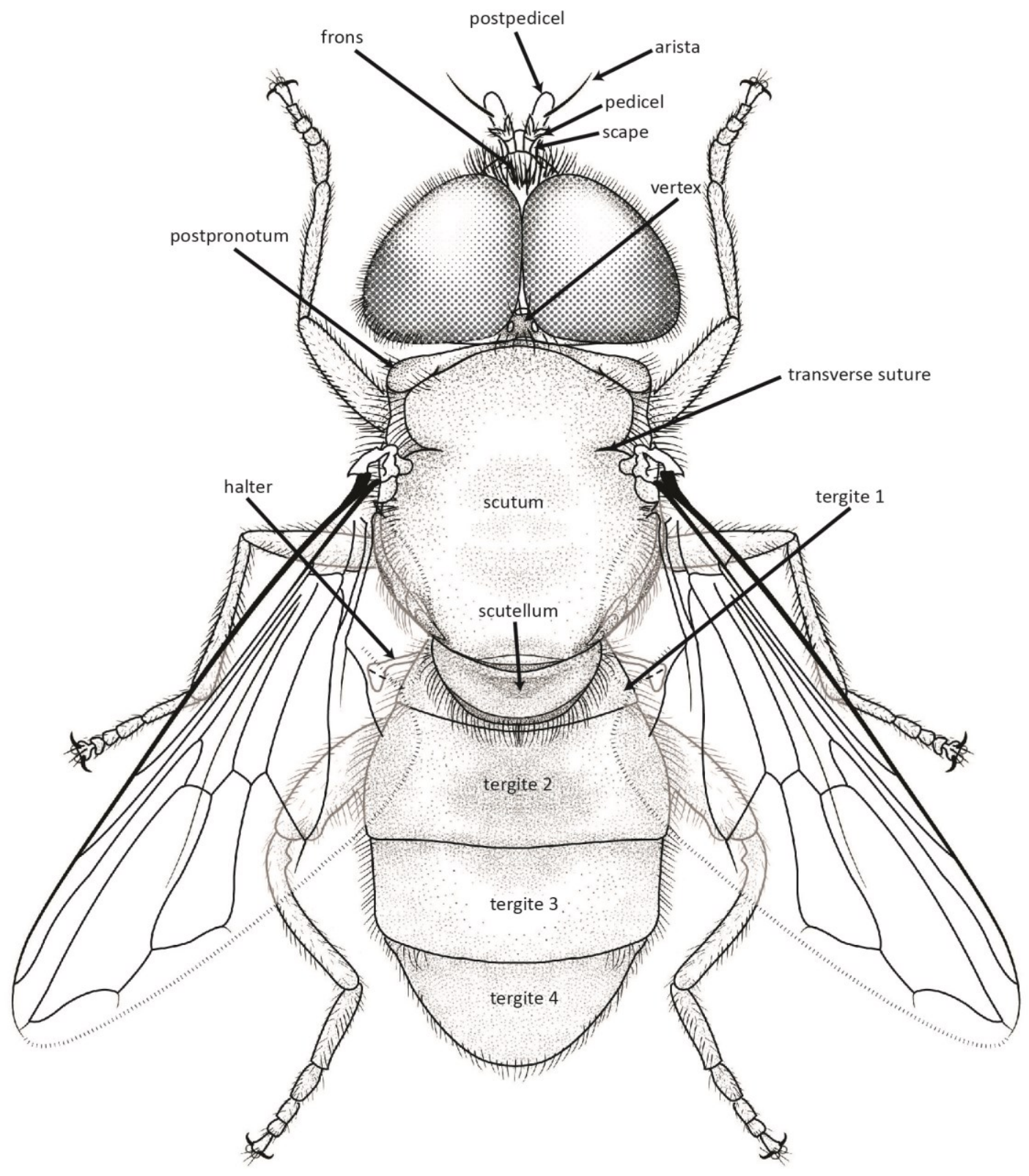

Chapter 3, Figure 2. Psilota alexanderi, dorsal habitus. Illustration by Jessica Hsiung. 


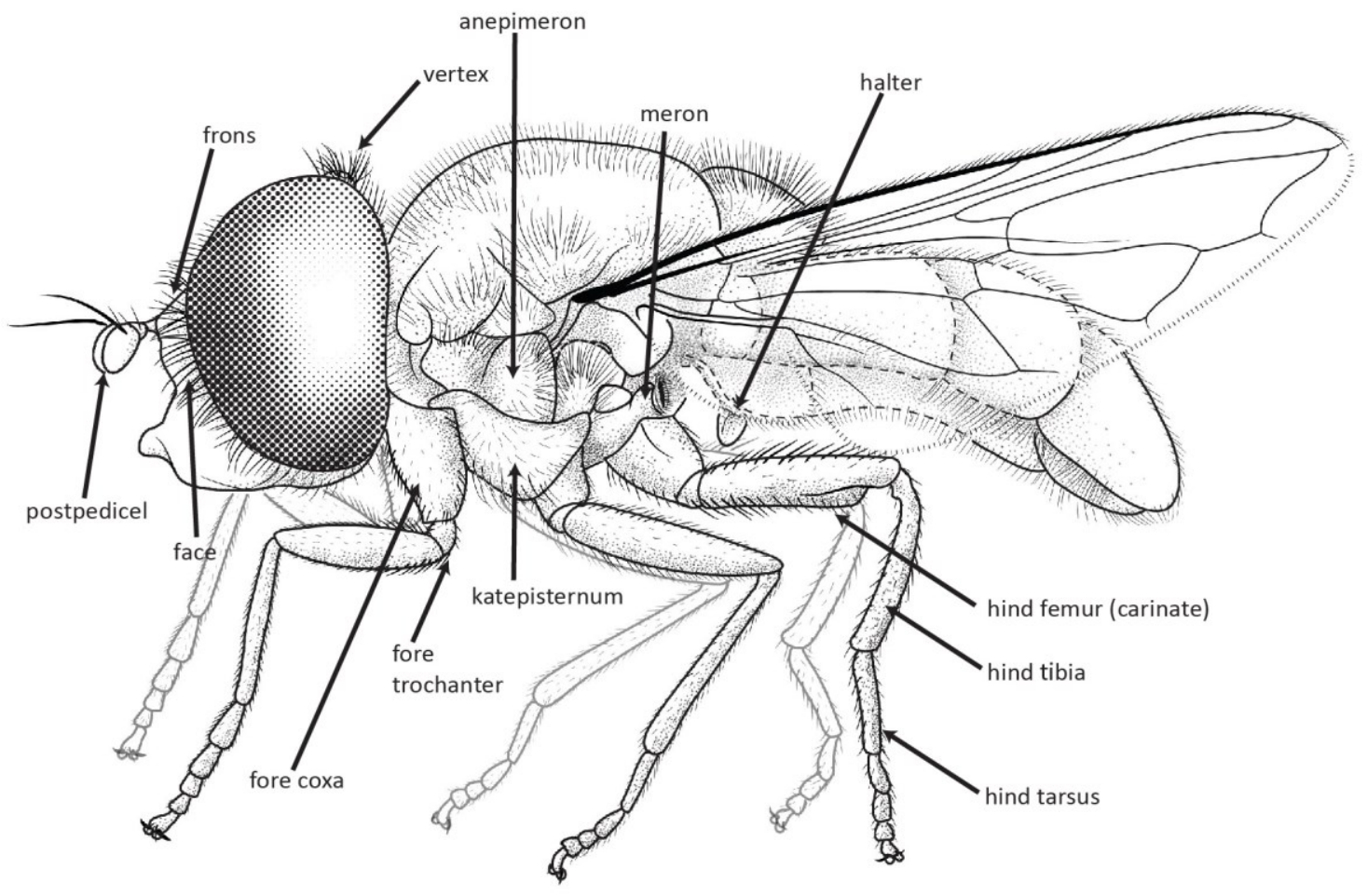

Chapter 3, Figure 3. Psilota alexanderi, lateral habitus. Illustration by Jessica Hsiung. 


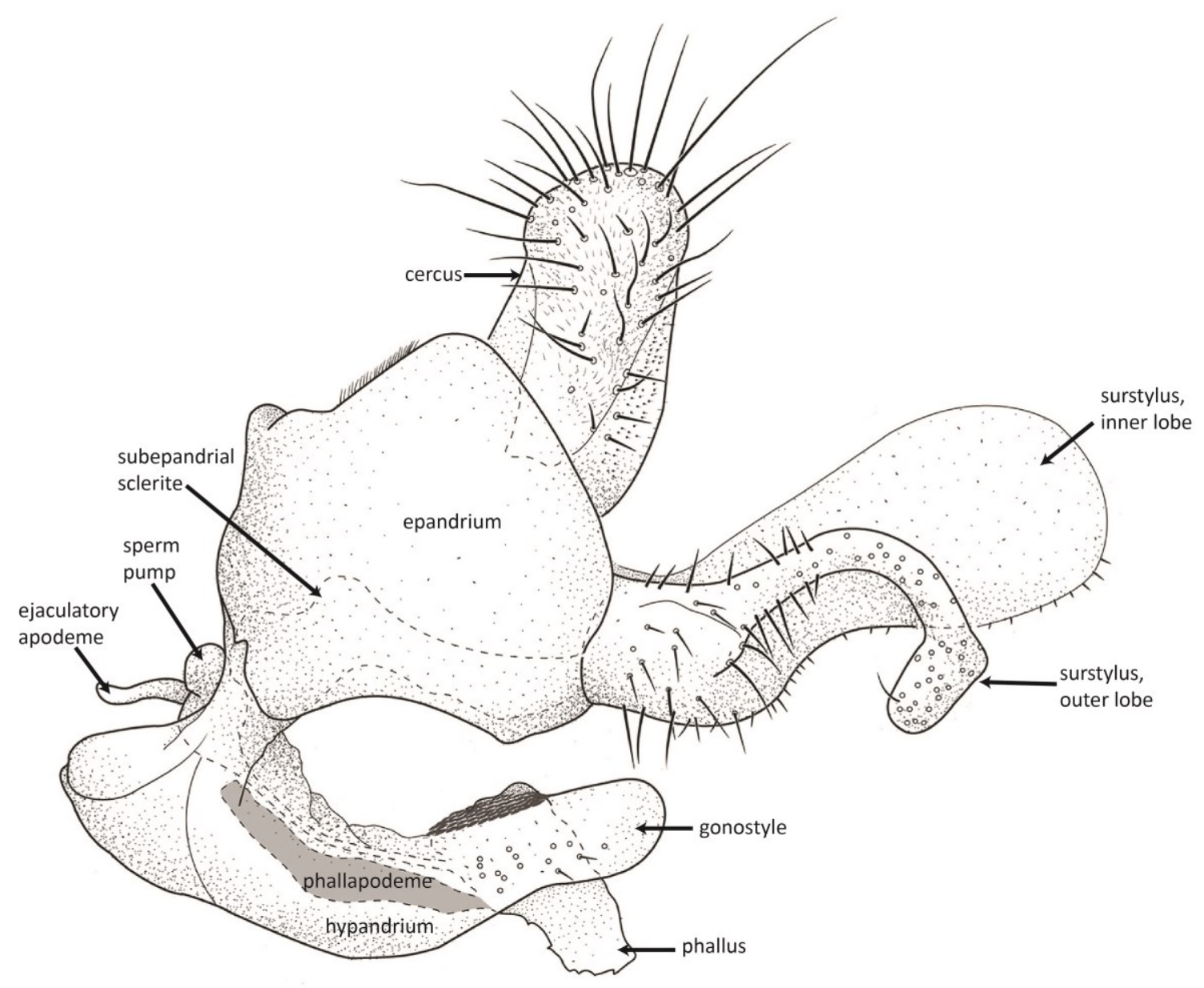

Chapter 3, Figure 4. Psilota alexanderi, genitalia lateral. Illustration by Jessica Hsiung. 


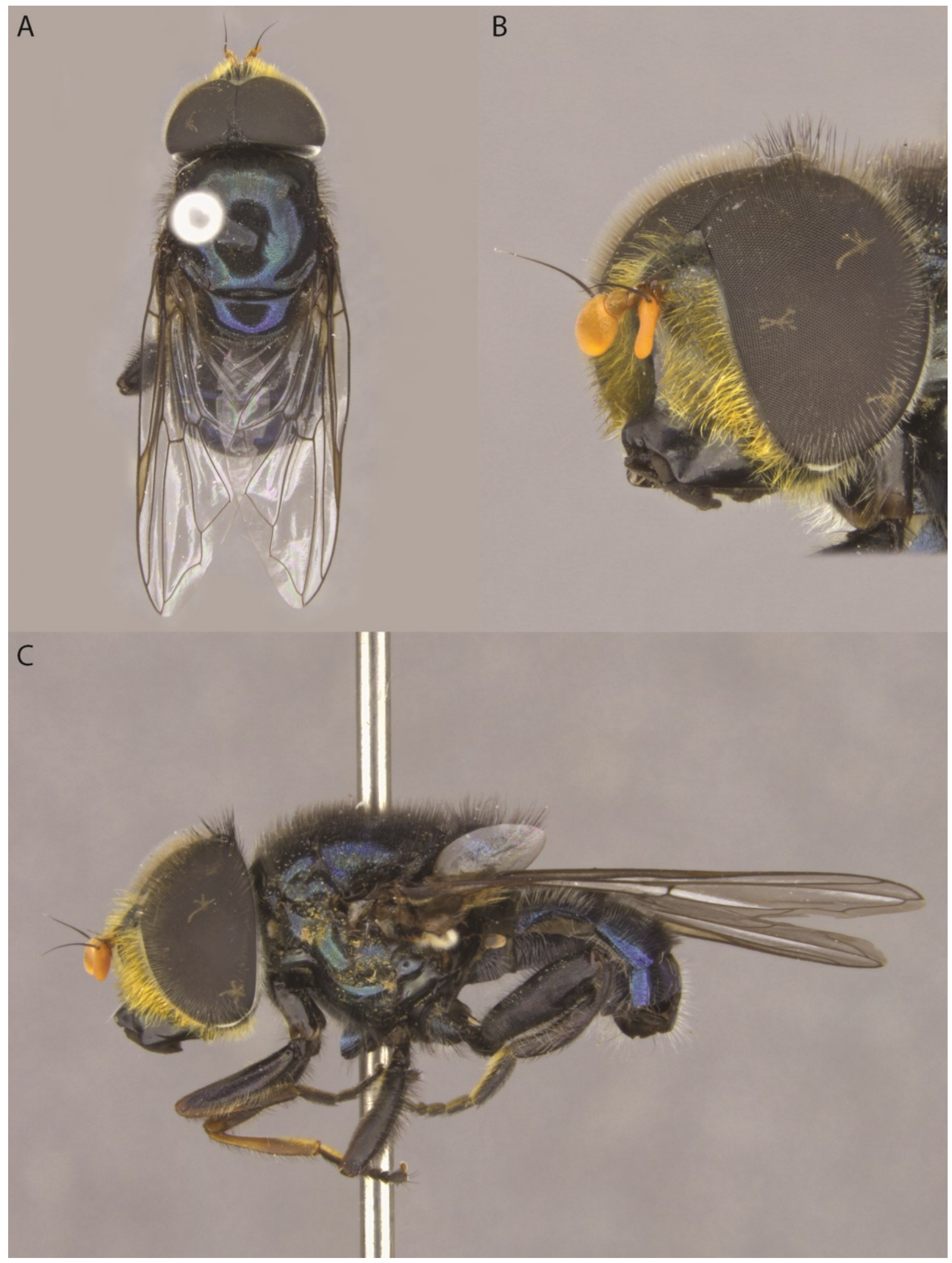

Chapter 3, Figure 5. P. aislinni. A) male dorsal habitus, B) male anterolateral face, C) male lateral habitus. 


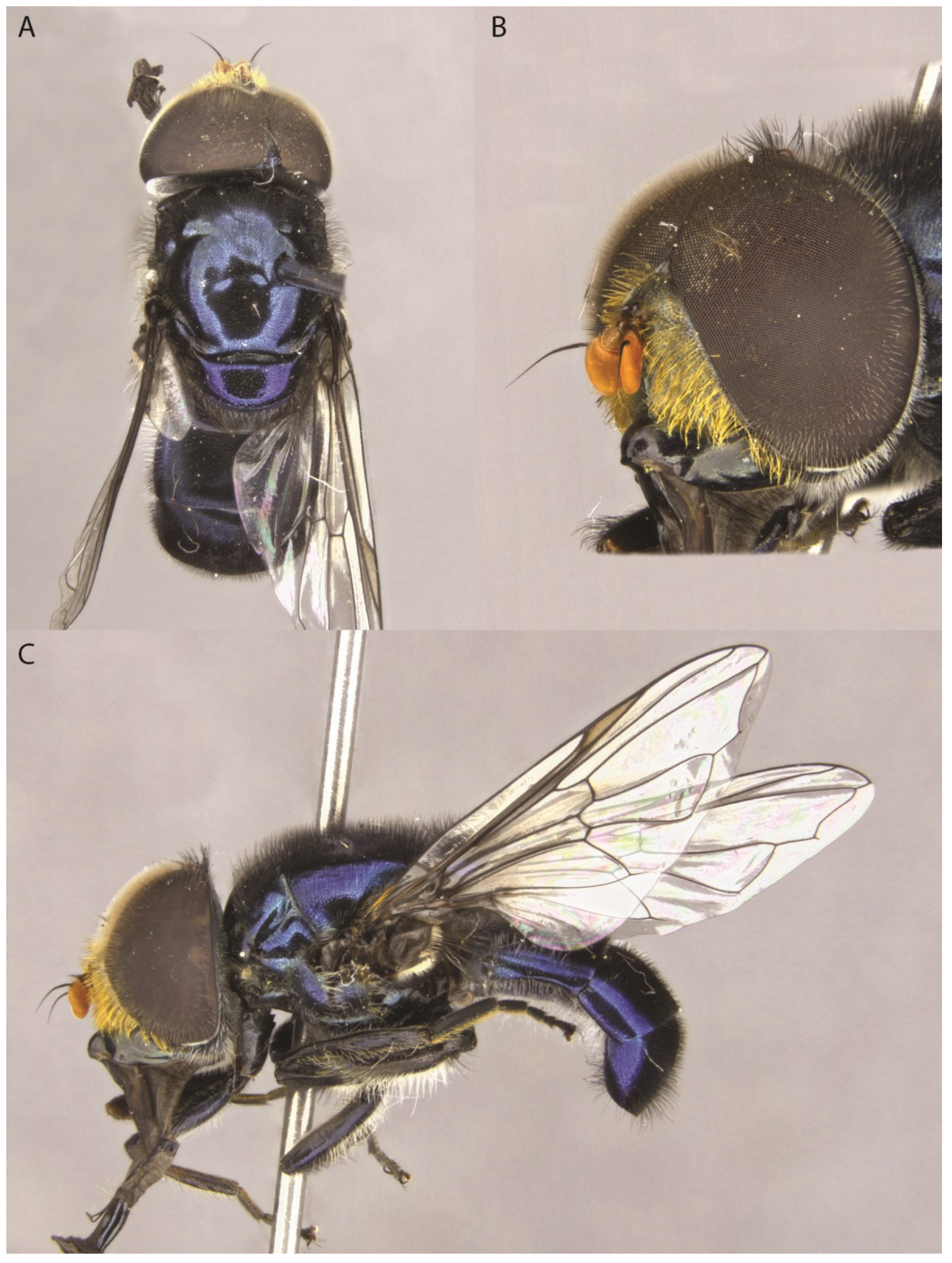

Chapter 3, Figure 6. P. alexanderi. A) male dorsal habitus, B) male anterolateral face, C) male lateral habitus. 


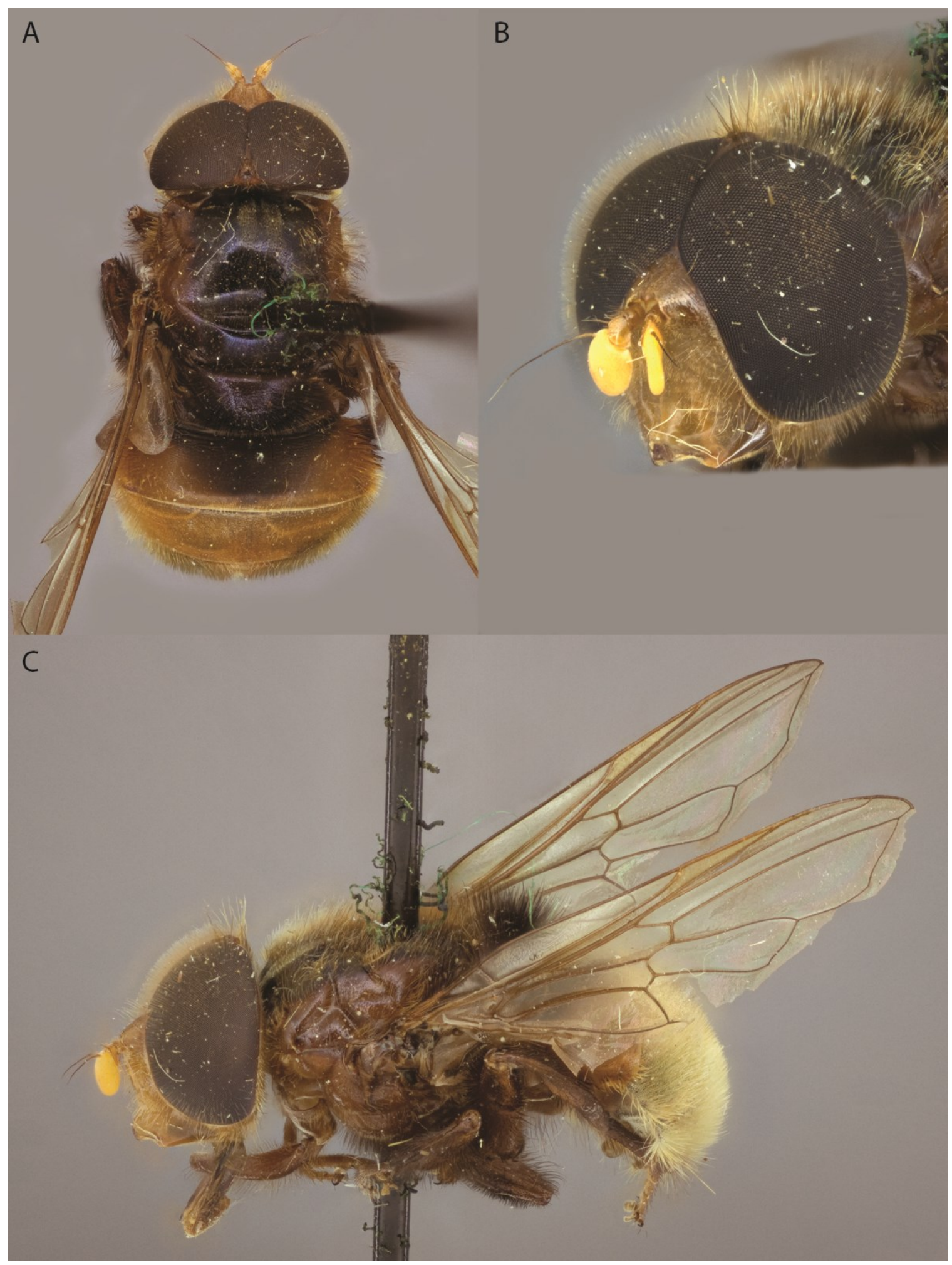

Chapter 3, Figure 7. P. apiformis. A) male dorsal habitus, B) male anterolateral face, C) male lateral habitus. 


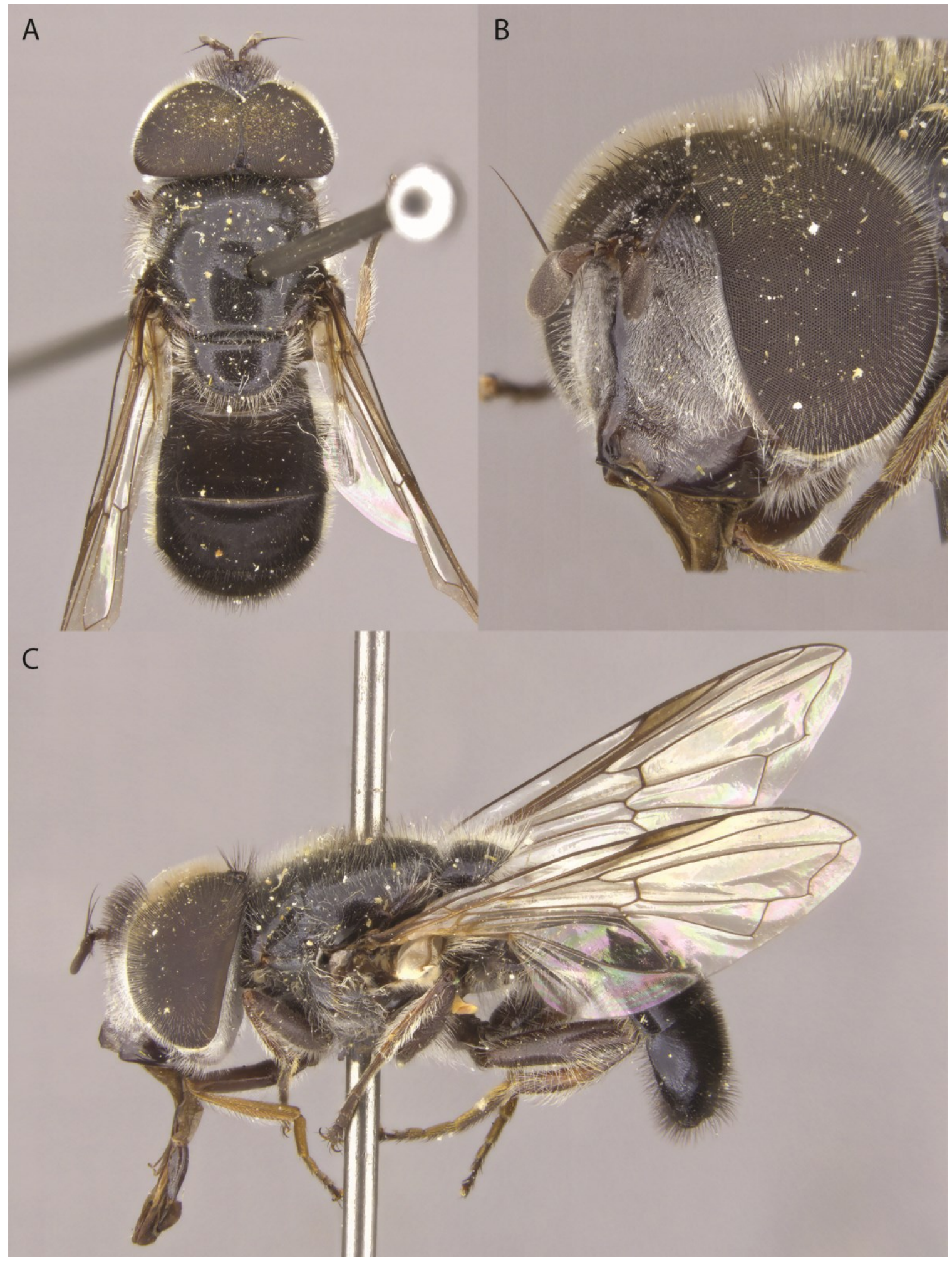

Chapter 3, Figure 8. P. auricauda. A) male dorsal habitus, B) male anterolateral face, C) male lateral habitus. 


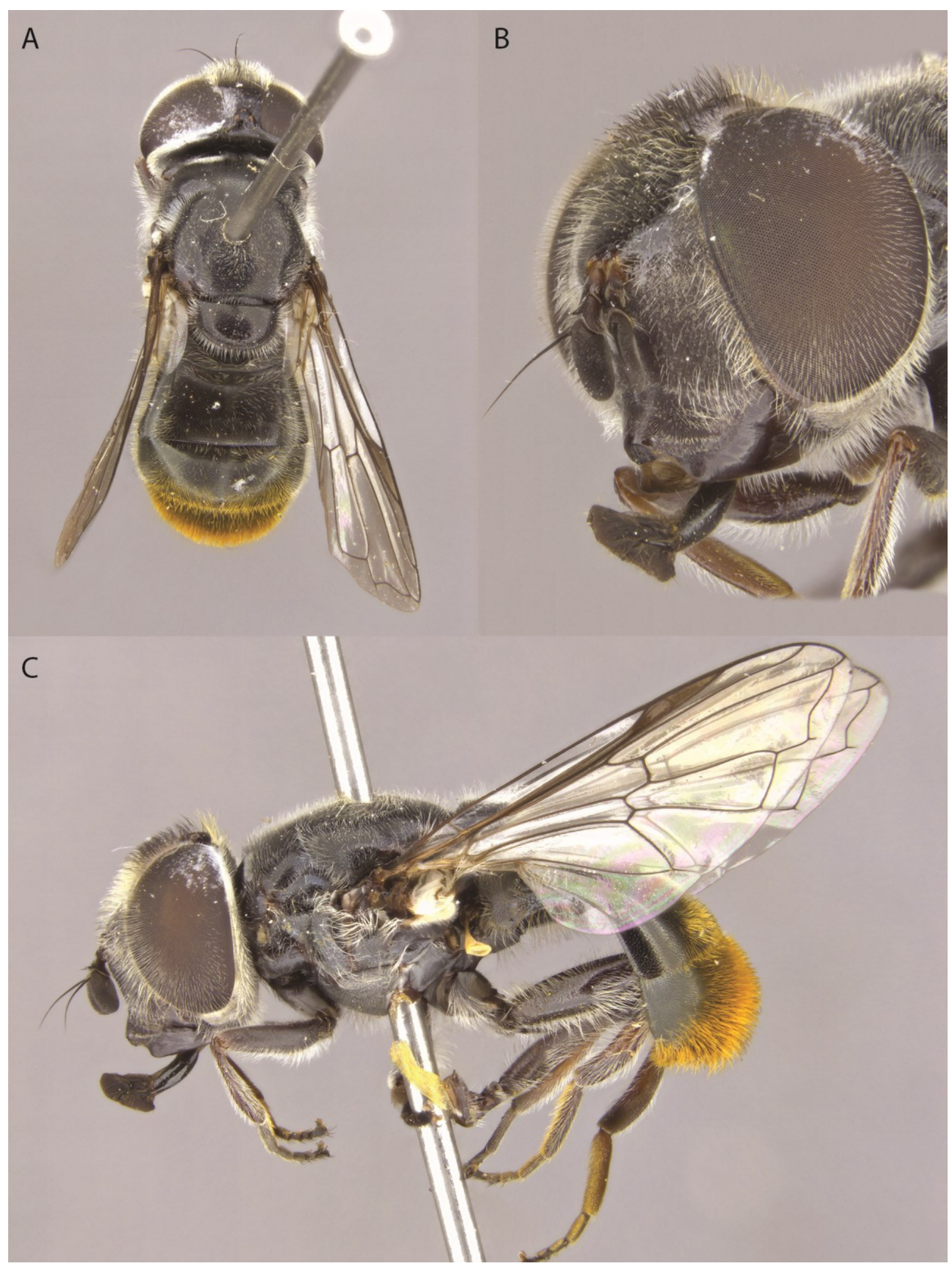

Chapter 3, Figure 9. P. auricauda. A) female dorsal habitus, B) female anterolateral face, C) female lateral habitus. 


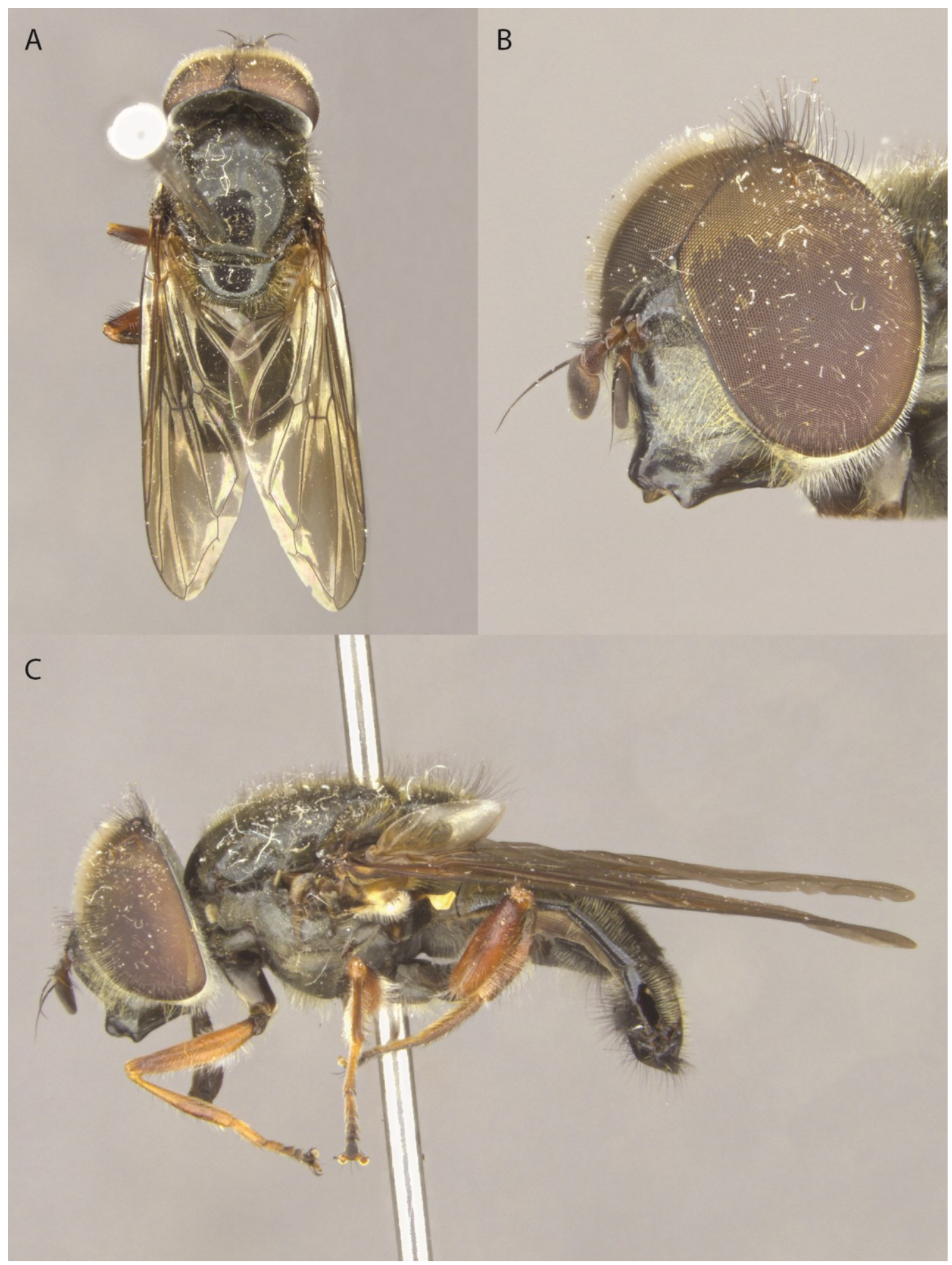

Chapter 3, Figure 10. P. auripilosa. A) male dorsal habitus, B) male anterolateral face, C) male lateral habitus. 


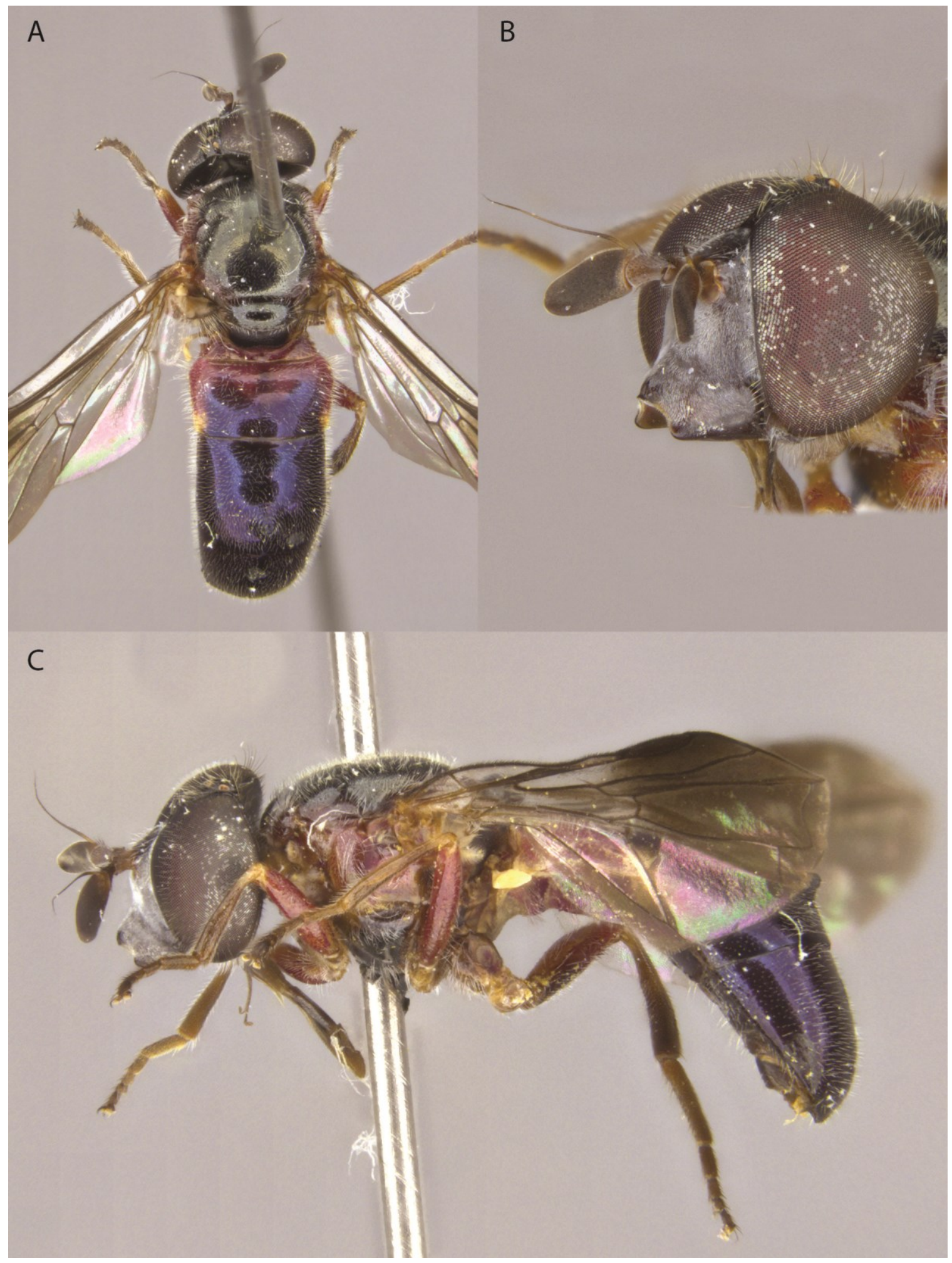

Chapter 3, Figure 11. P. austropsilota. A) male dorsal habitus, B) male anterolateral face, C) male lateral habitus. 


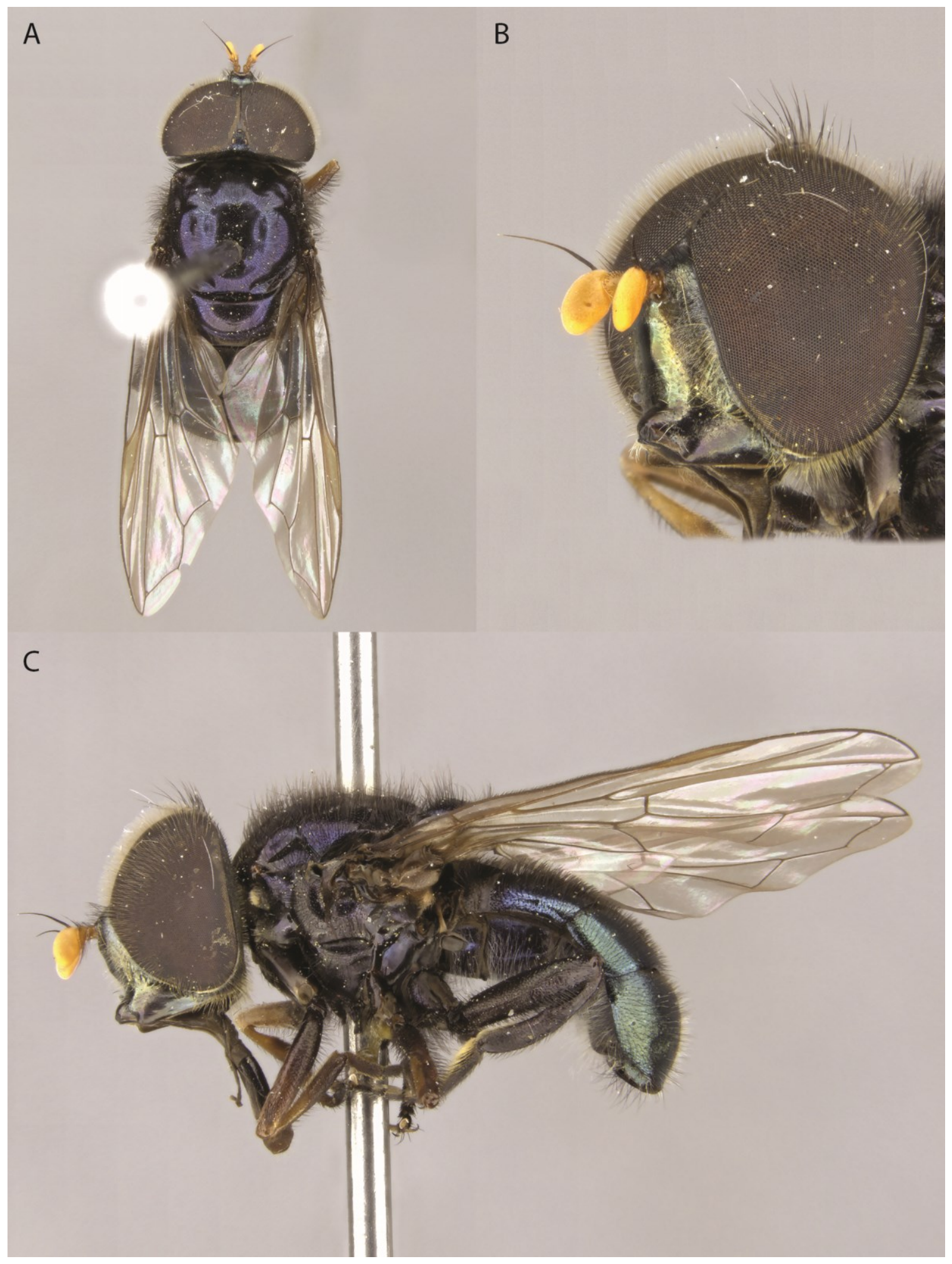

Chapter 3, Figure 12. P. azurea. A) male dorsal habitus, B) male anterolateral face, C) male lateral habitus. 


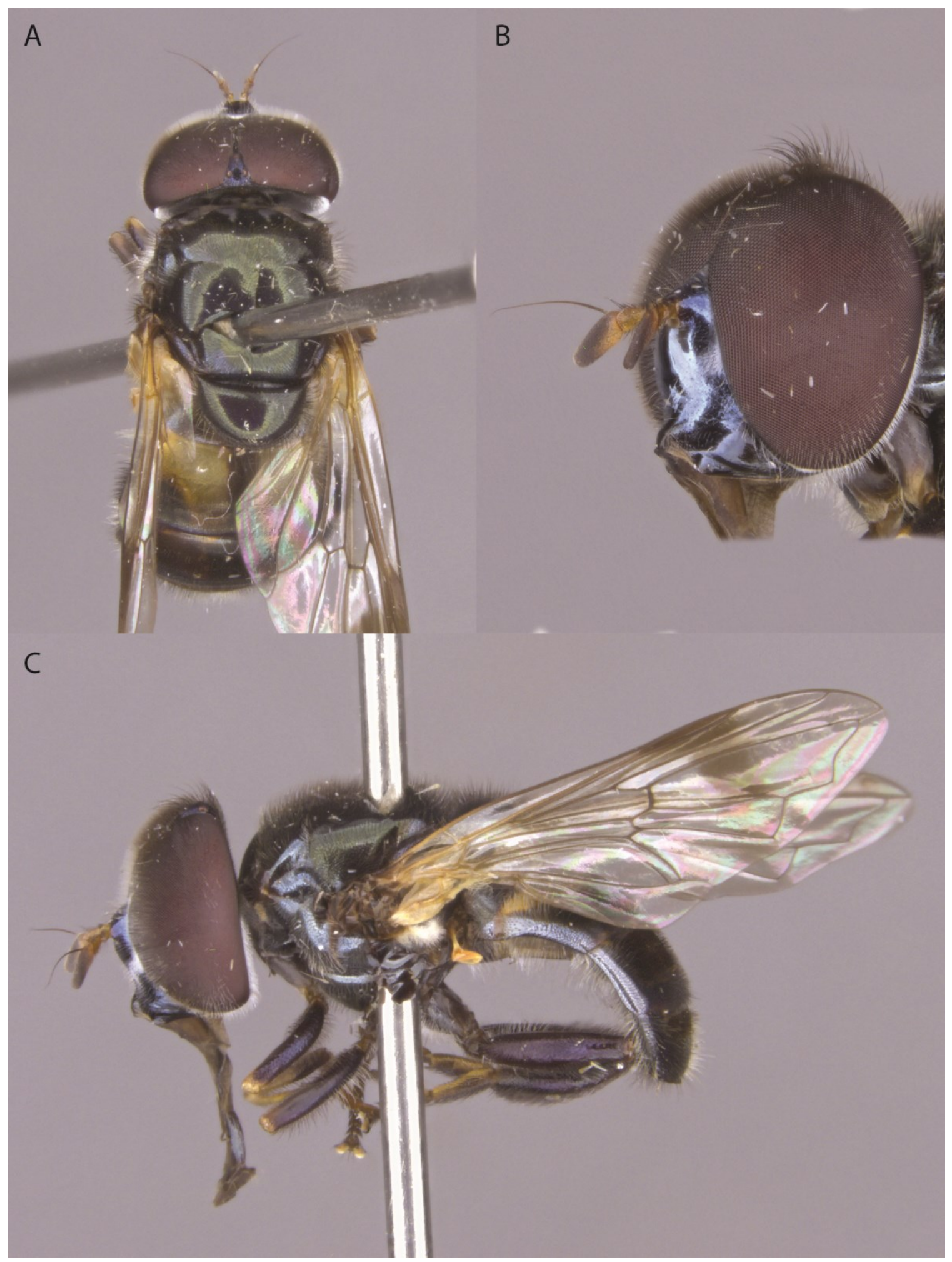

Chapter 3, Figure 13. P. basalis. A) male dorsal habitus, B) male anterolateral face, C) male lateral habitus. 


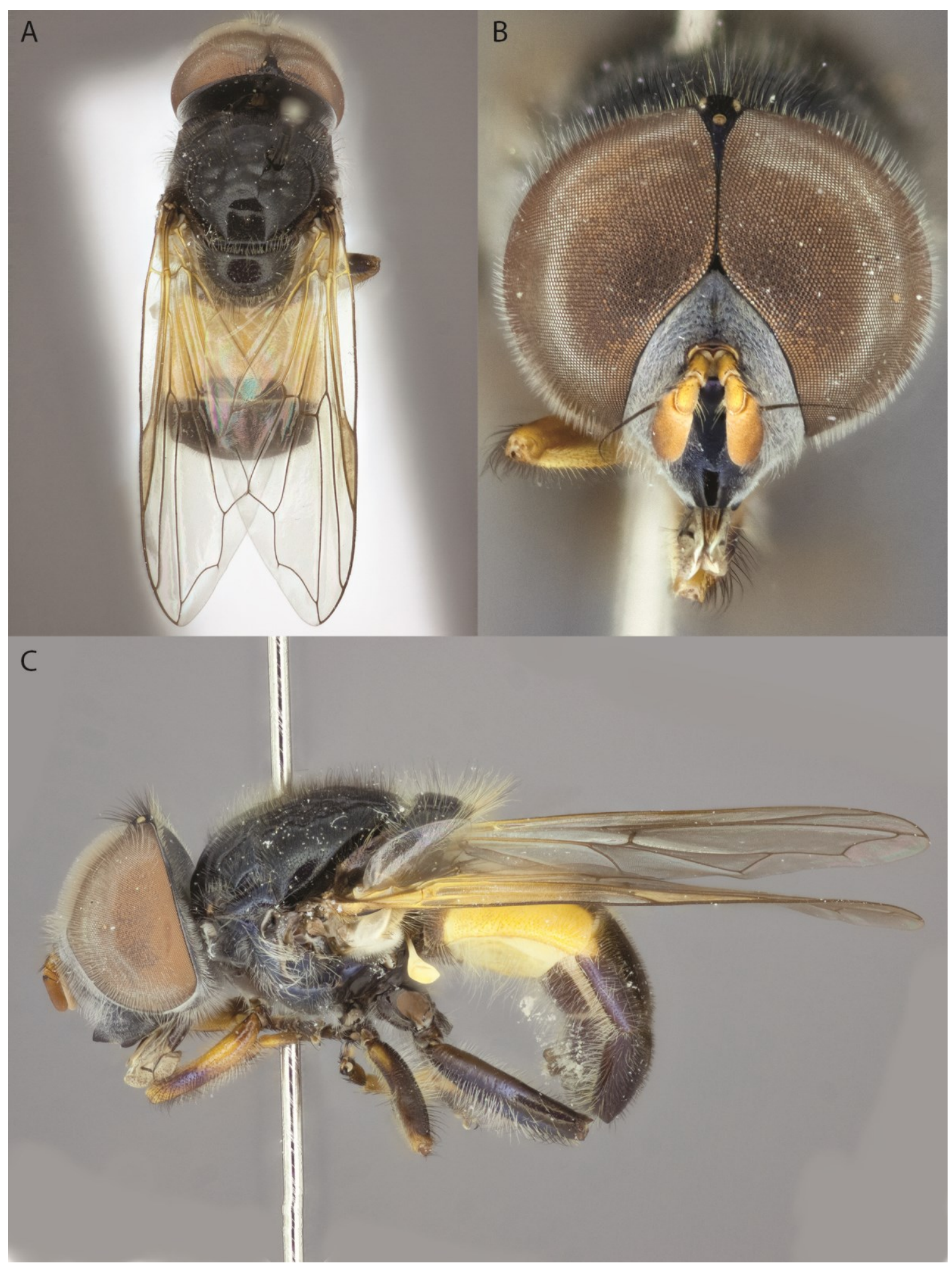

Chapter 3, Figure 14. P. bicolor. A) male dorsal habitus, B) male anterolateral face, C) male lateral habitus. 


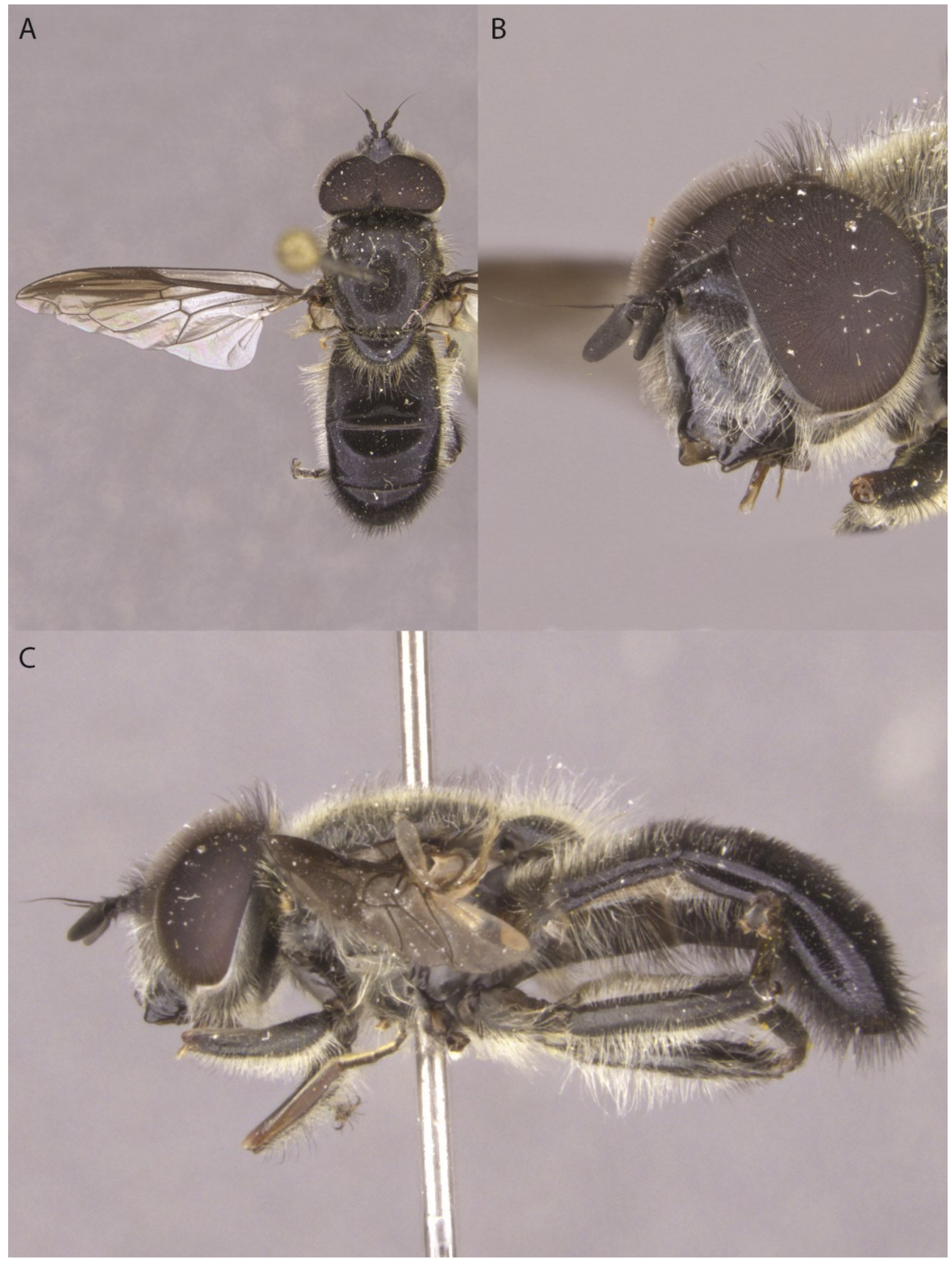

Chapter 3, Figure 15. P. brunnipennis. A) male dorsal habitus, B) male anterolateral face, C) male lateral habitus. 


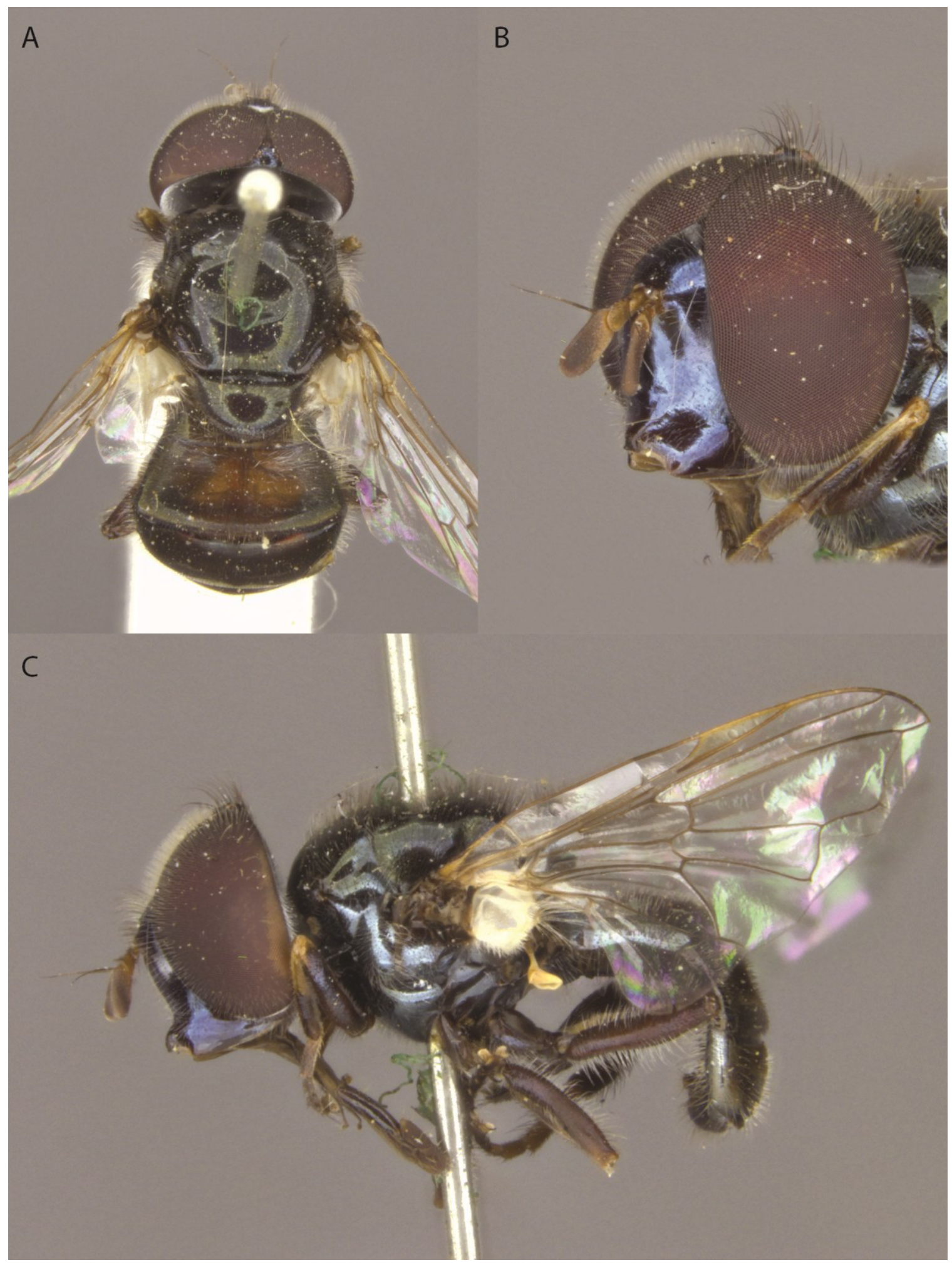

Chapter 3, Figure 16. P. calva. A) male dorsal habitus, B) male anterolateral face, C) male lateral habitus. 


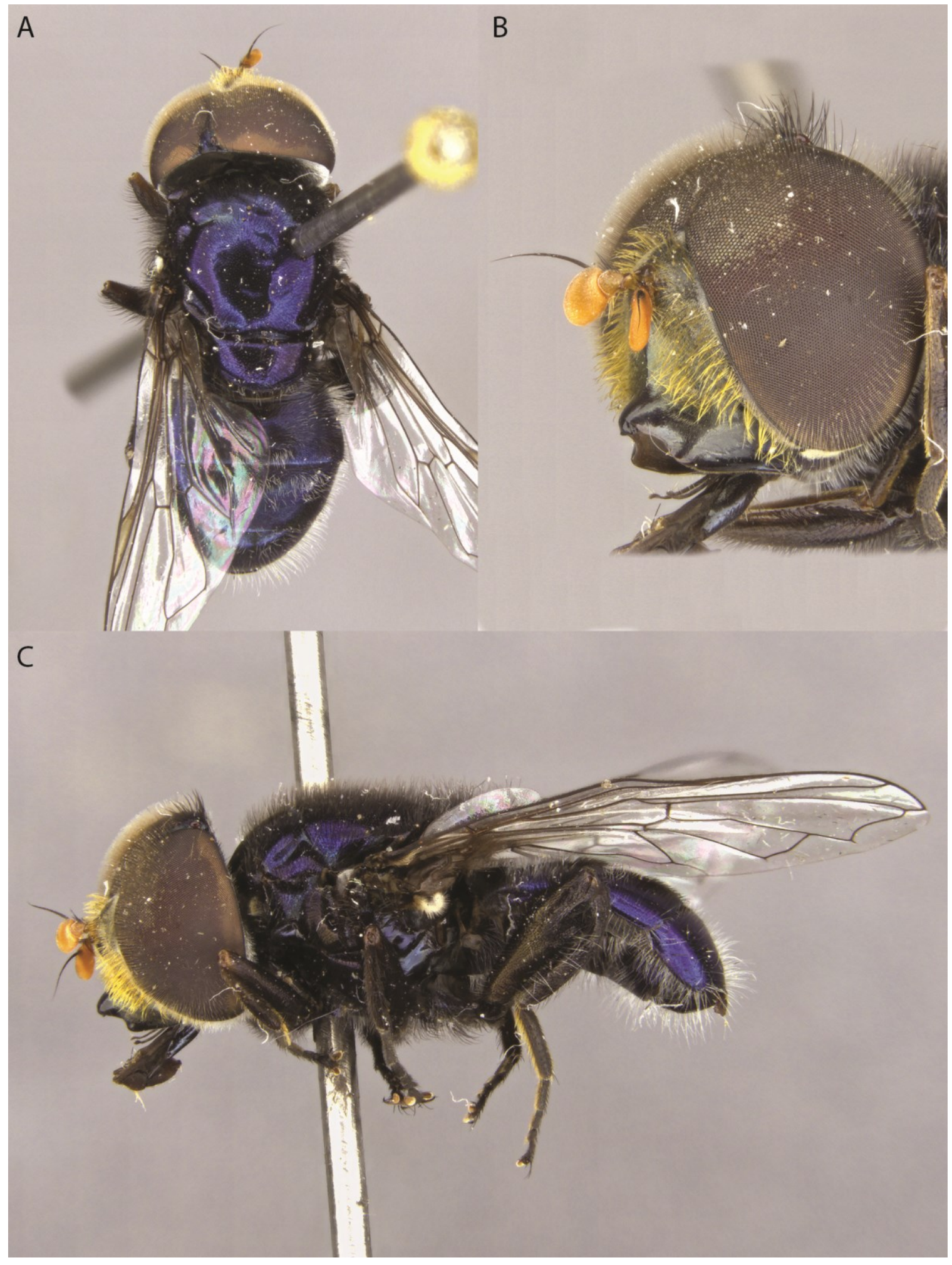

Chapter 3, Figure 17. P. coerulea. A) male dorsal habitus, B) male anterolateral face, C) male lateral habitus. 


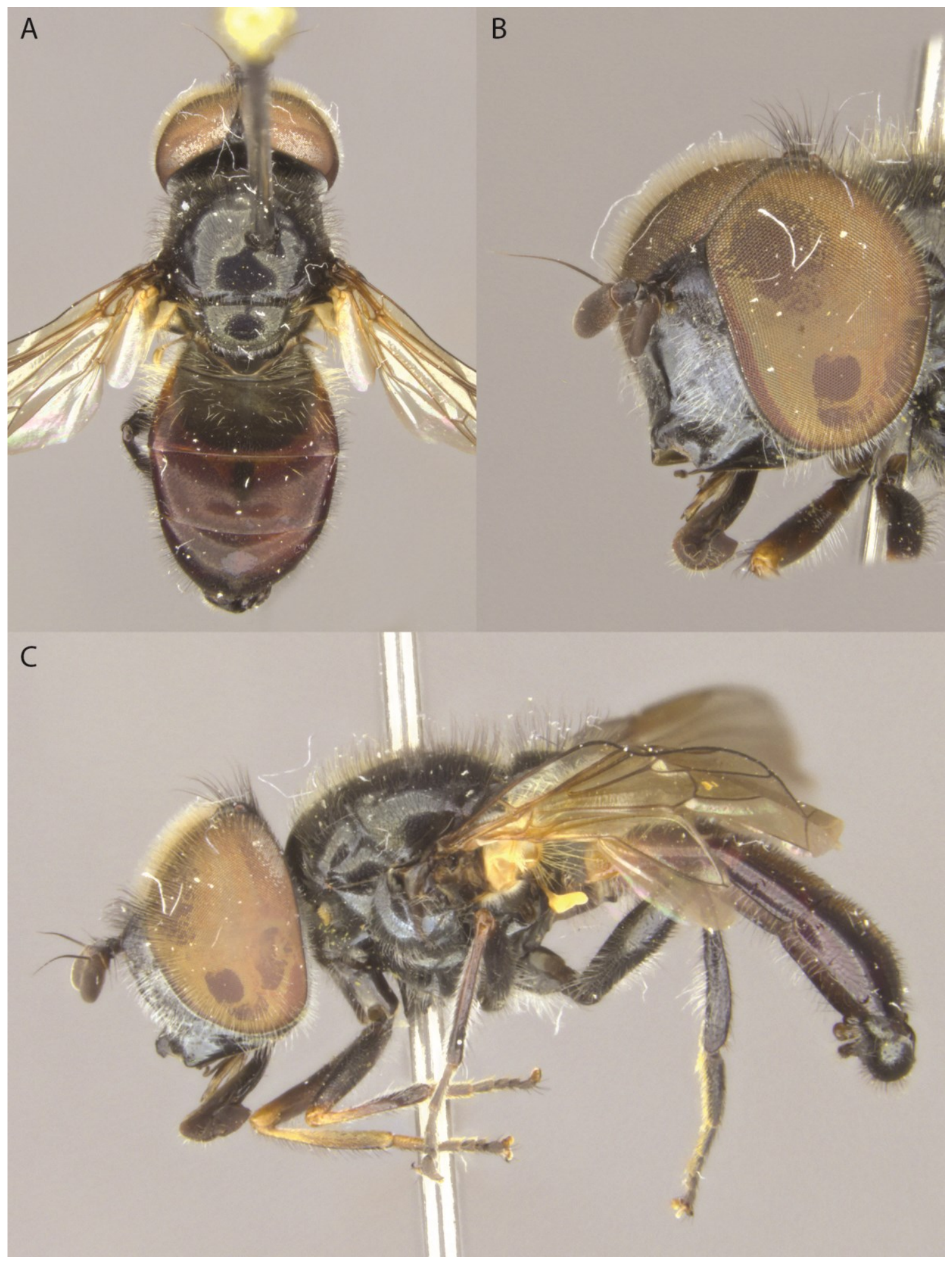

Chapter 3, Figure 18. P. cupreus. A) male dorsal habitus, B) male anterolateral face, C) male lateral habitus. 


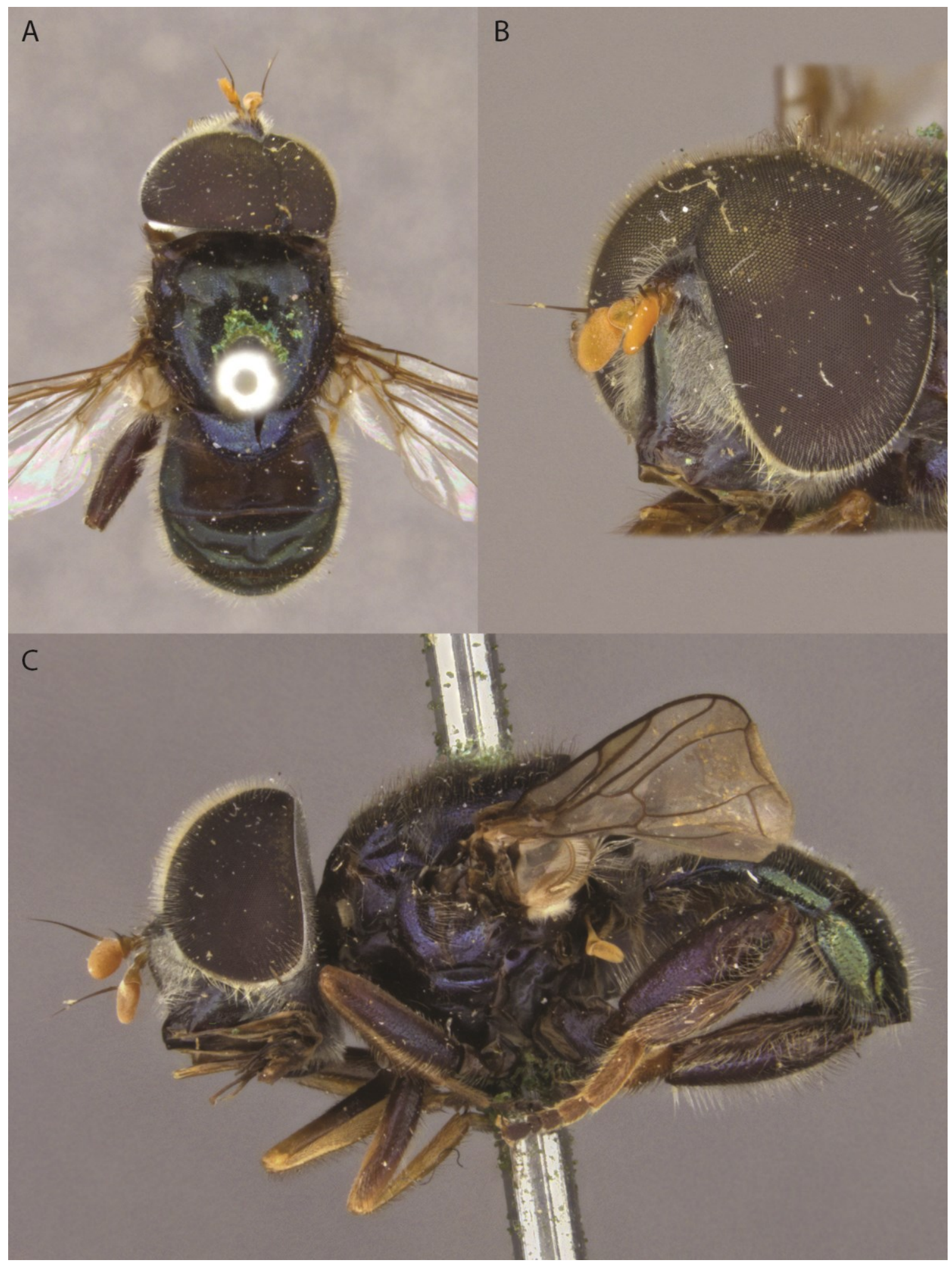

Chapter 3, Figure 19. P. darwini. A) male dorsal habitus, B) male anterolateral face, C) male lateral habitus. 


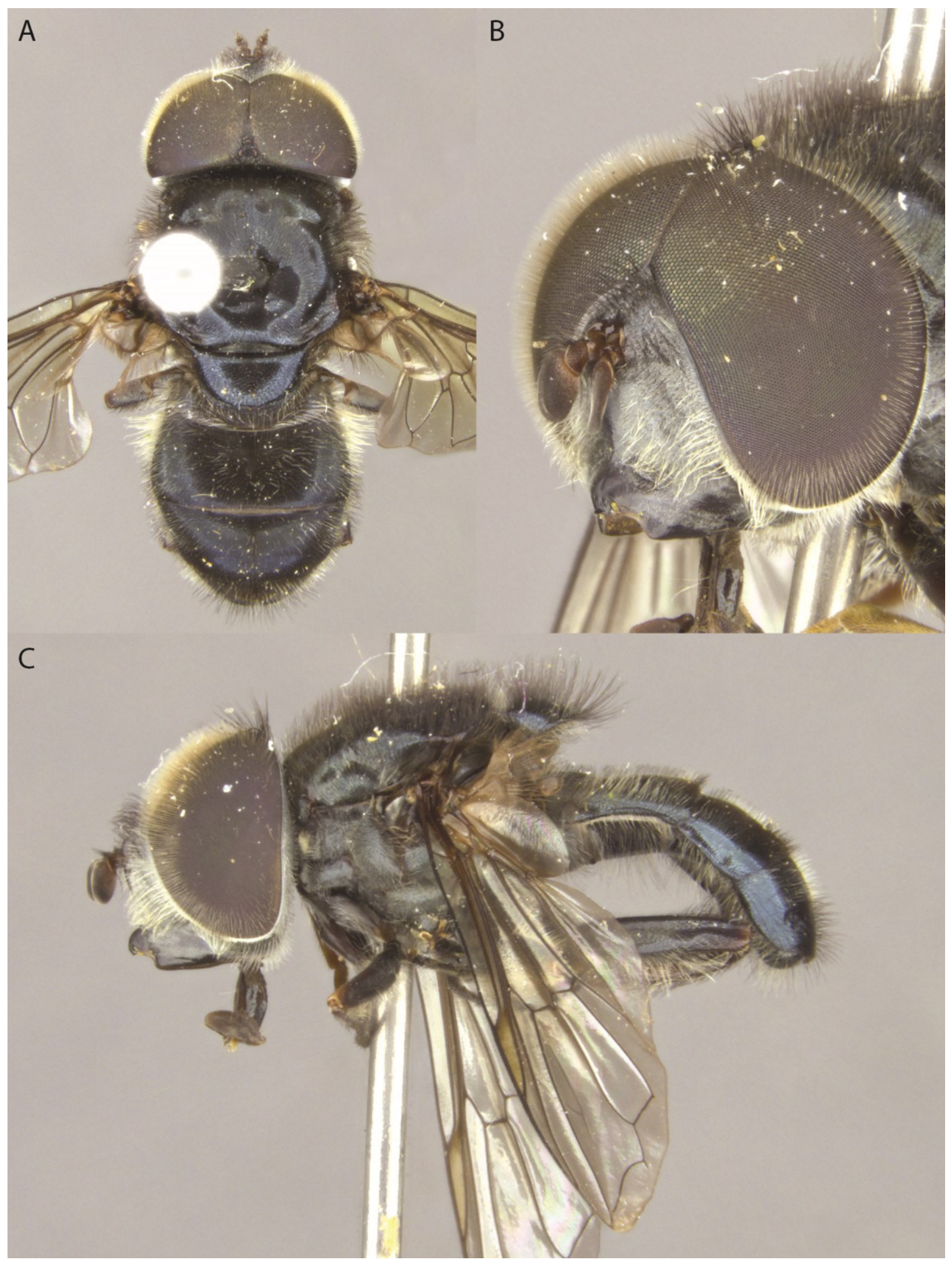

Chapter 3, Figure 20. P. femoralis. A) male dorsal habitus, B) male anterolateral face, C) male lateral habitus. 


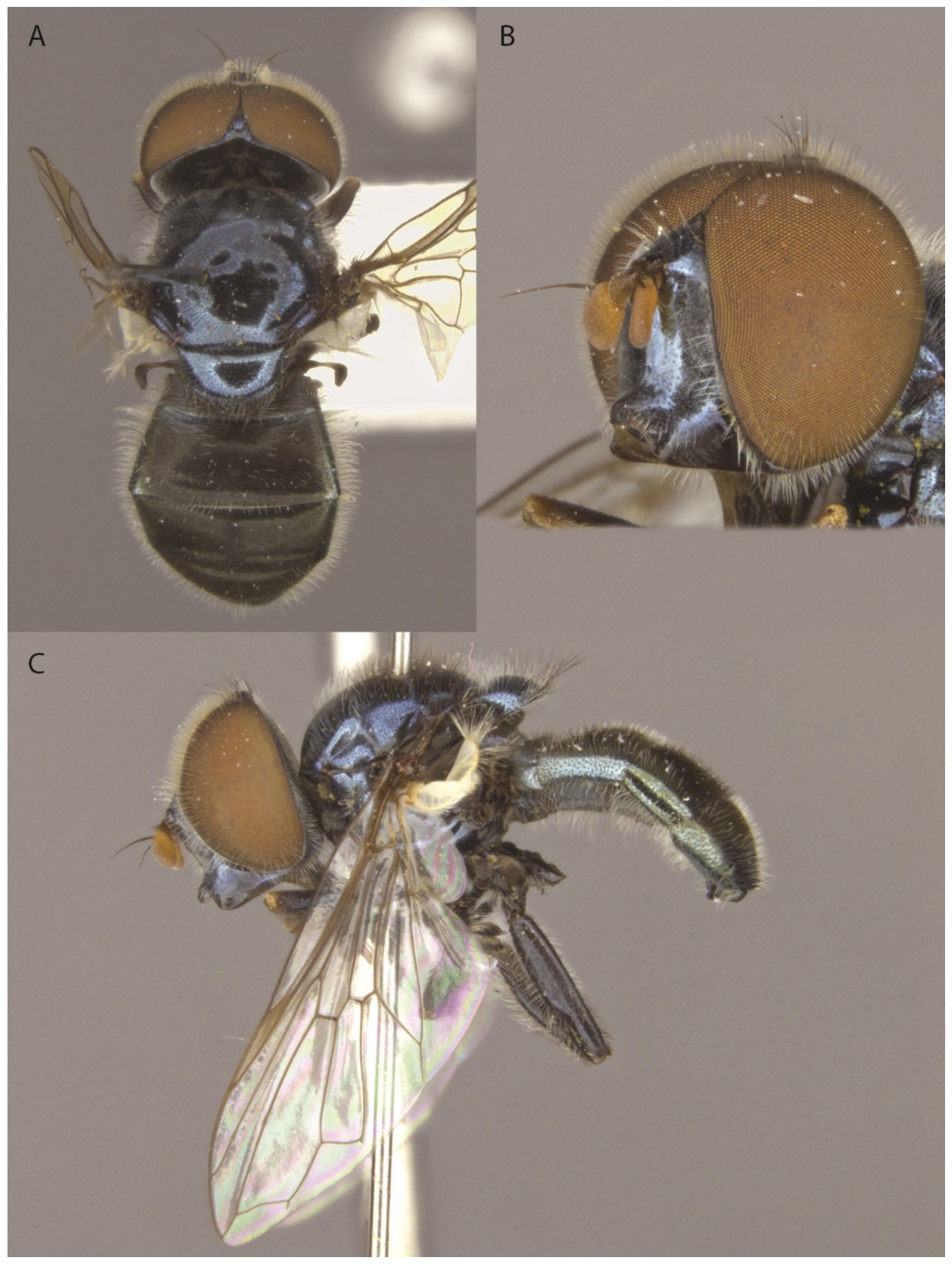

Chapter 3, Figure 21. P. lividus. A) male dorsal habitus, B) male anterolateral face, C) male lateral habitus. 


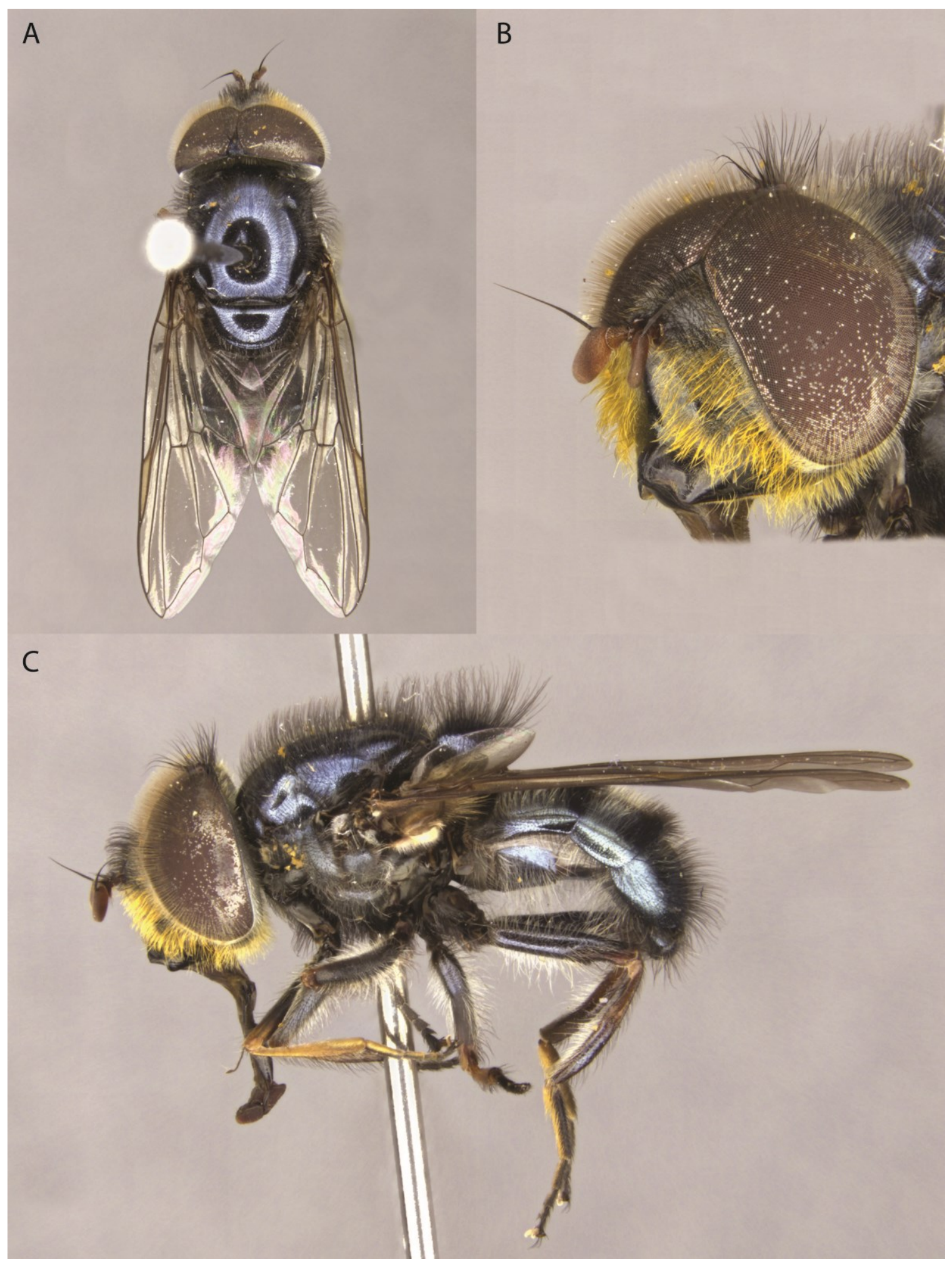

Chapter 3, Figure 22. P. longipilosa. A) male dorsal habitus, B) male anterolateral face, C) male lateral habitus. 


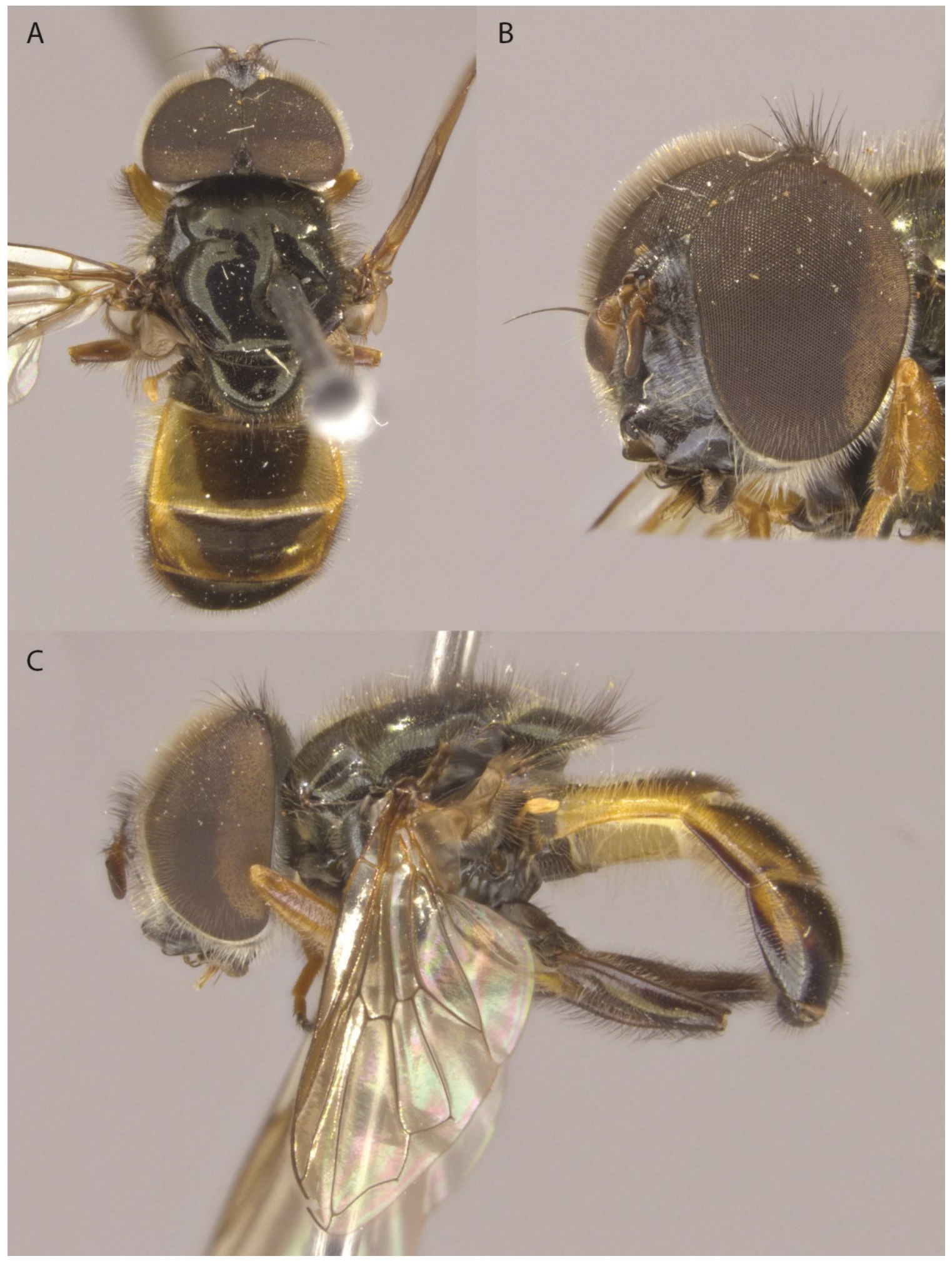

Chapter 3, Figure 23. P. maculata. A) male dorsal habitus, B) male anterolateral face, C) male lateral habitus. 


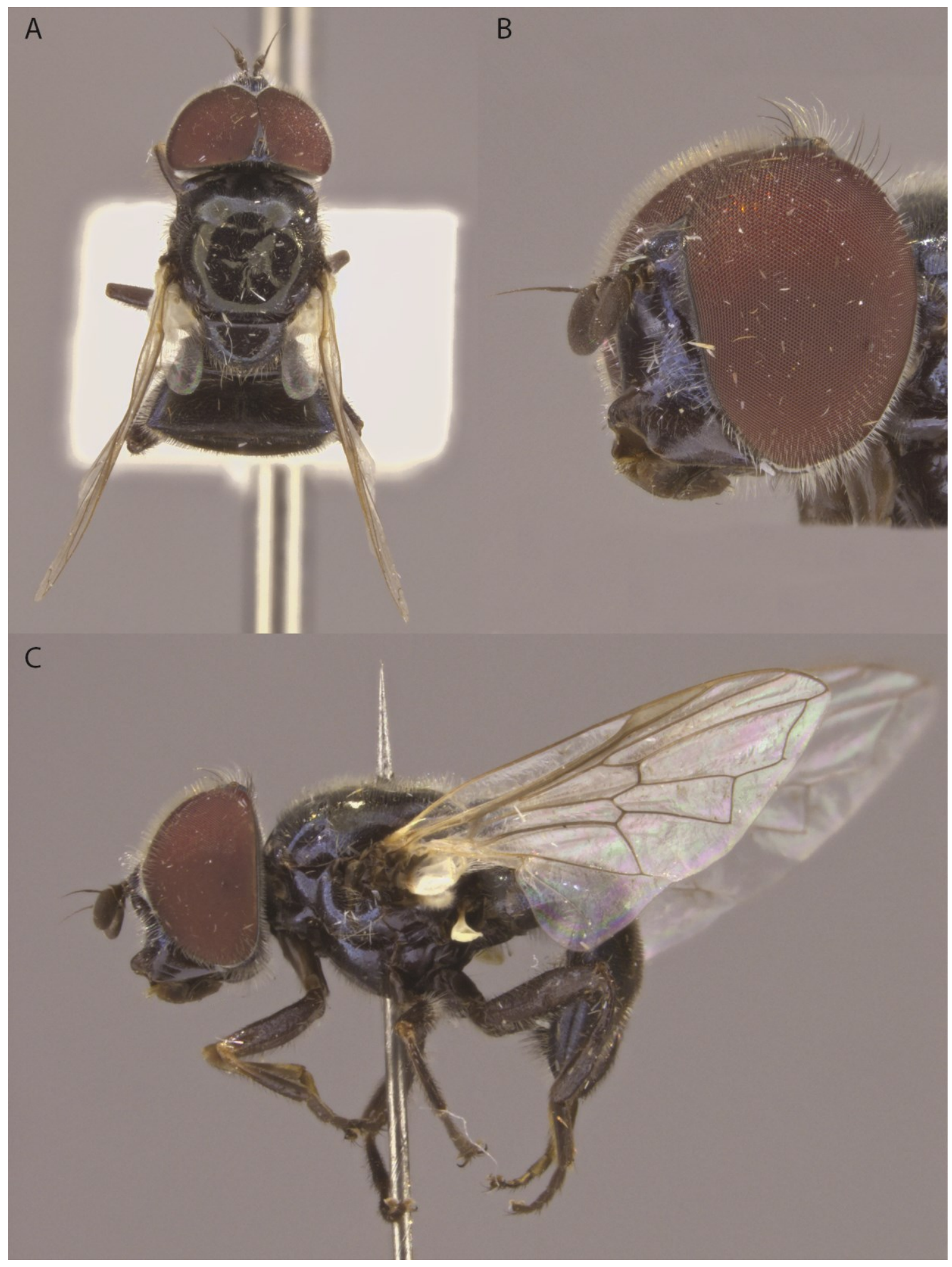

Chapter 3, Figure 24. P. mcqueeni. A) male dorsal habitus, B) male anterolateral face, C) male lateral habitus. 


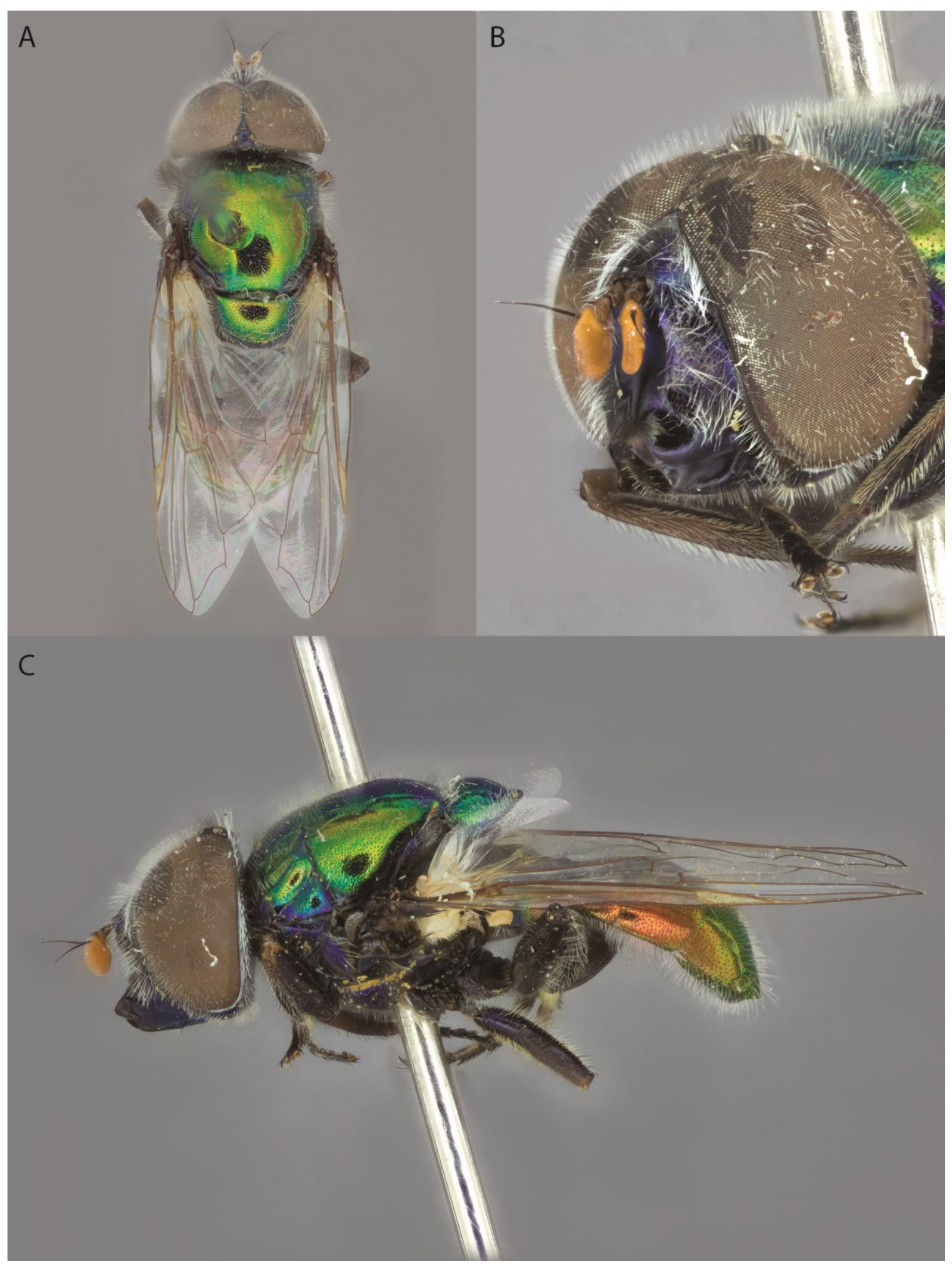

Chapter 3, Figure 25. P. metallica. A) male dorsal habitus, B) male anterolateral face, C) male lateral habitus. 


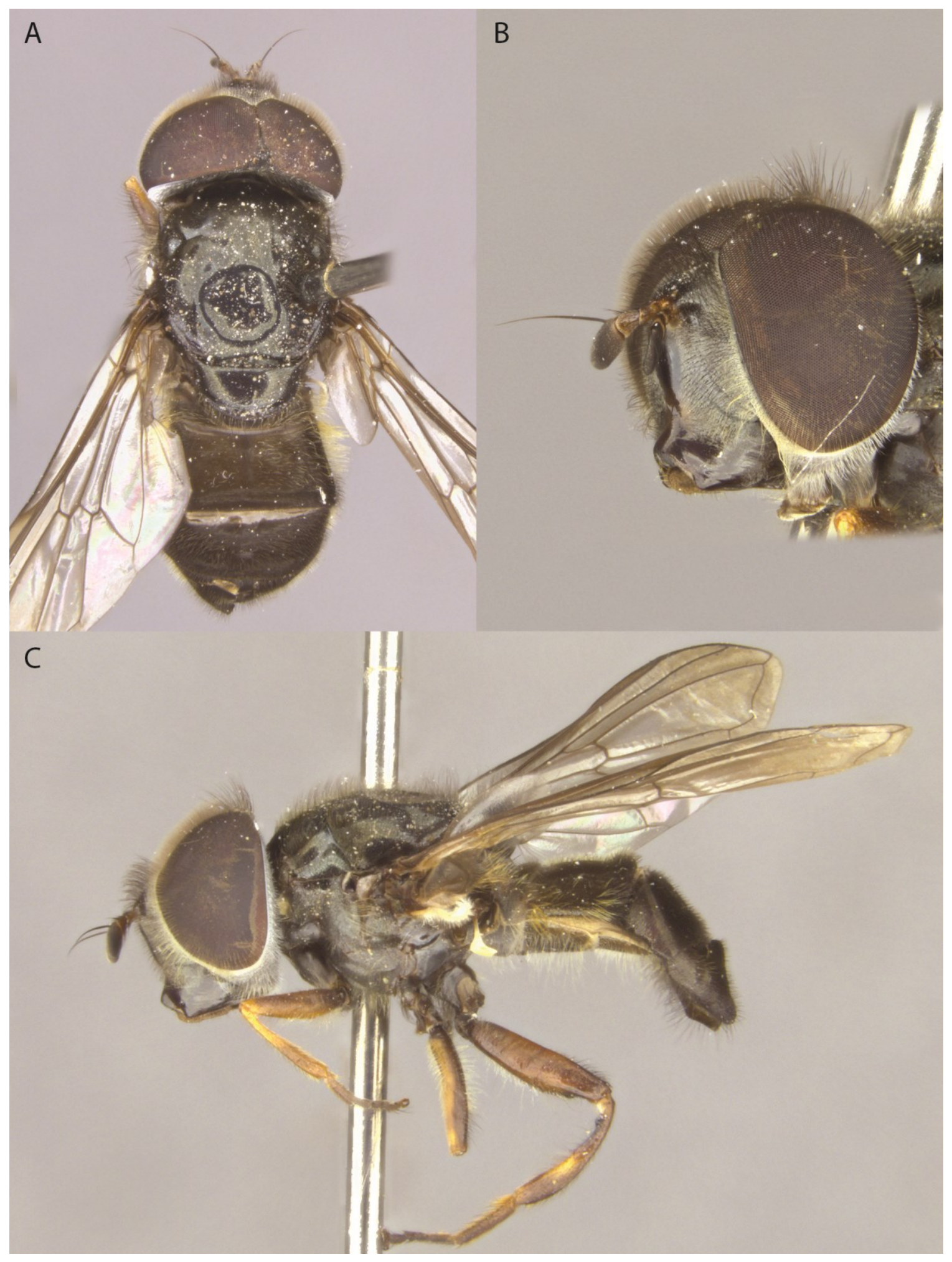

Chapter 3, Figure 26. P. nigripilosa. A) male dorsal habitus, B) male anterolateral face, C) male lateral habitus. 


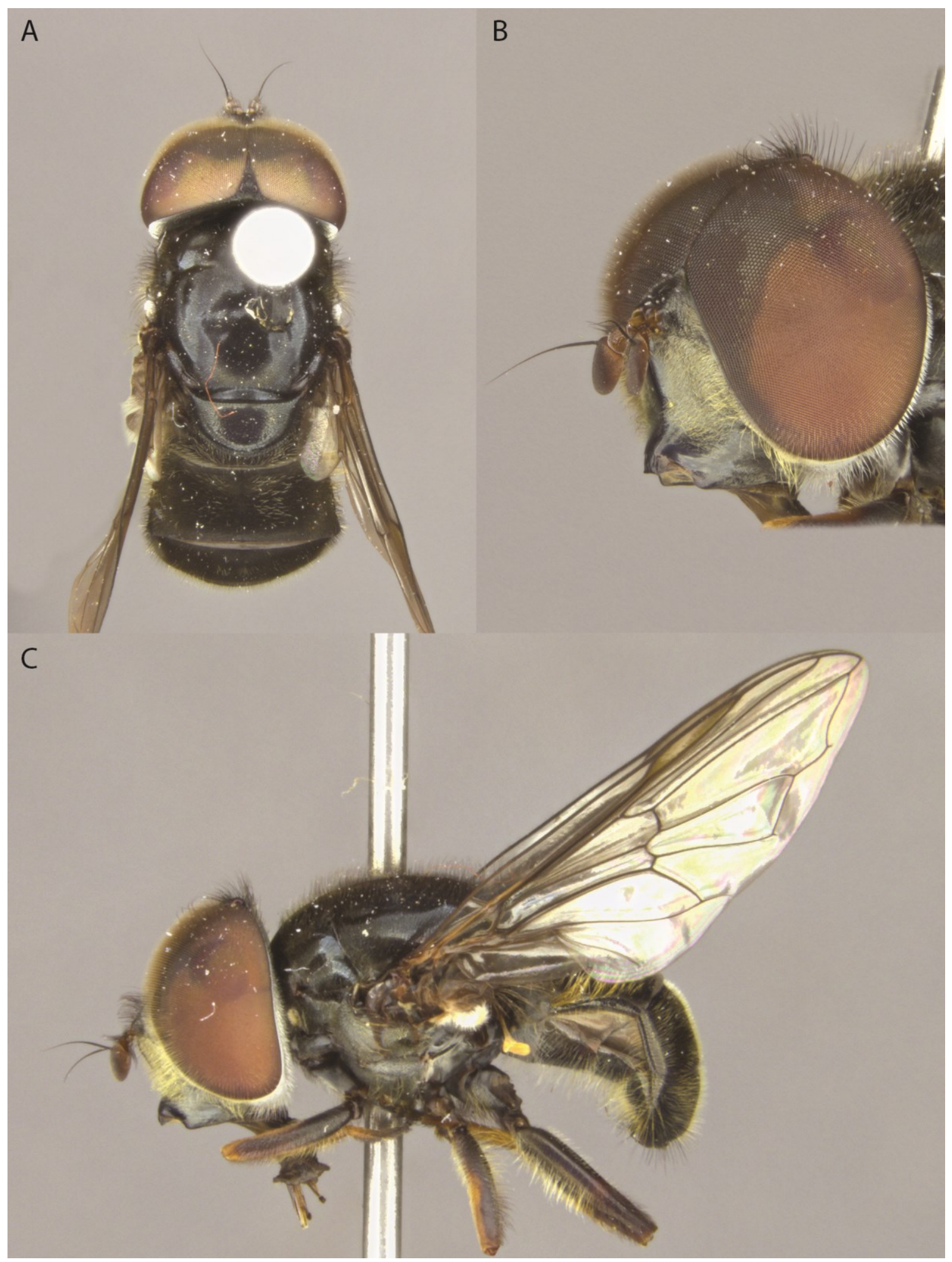

Chapter 3, Figure 27. P. nitida. A) male dorsal habitus, B) male anterolateral face, C) male lateral habitus. 


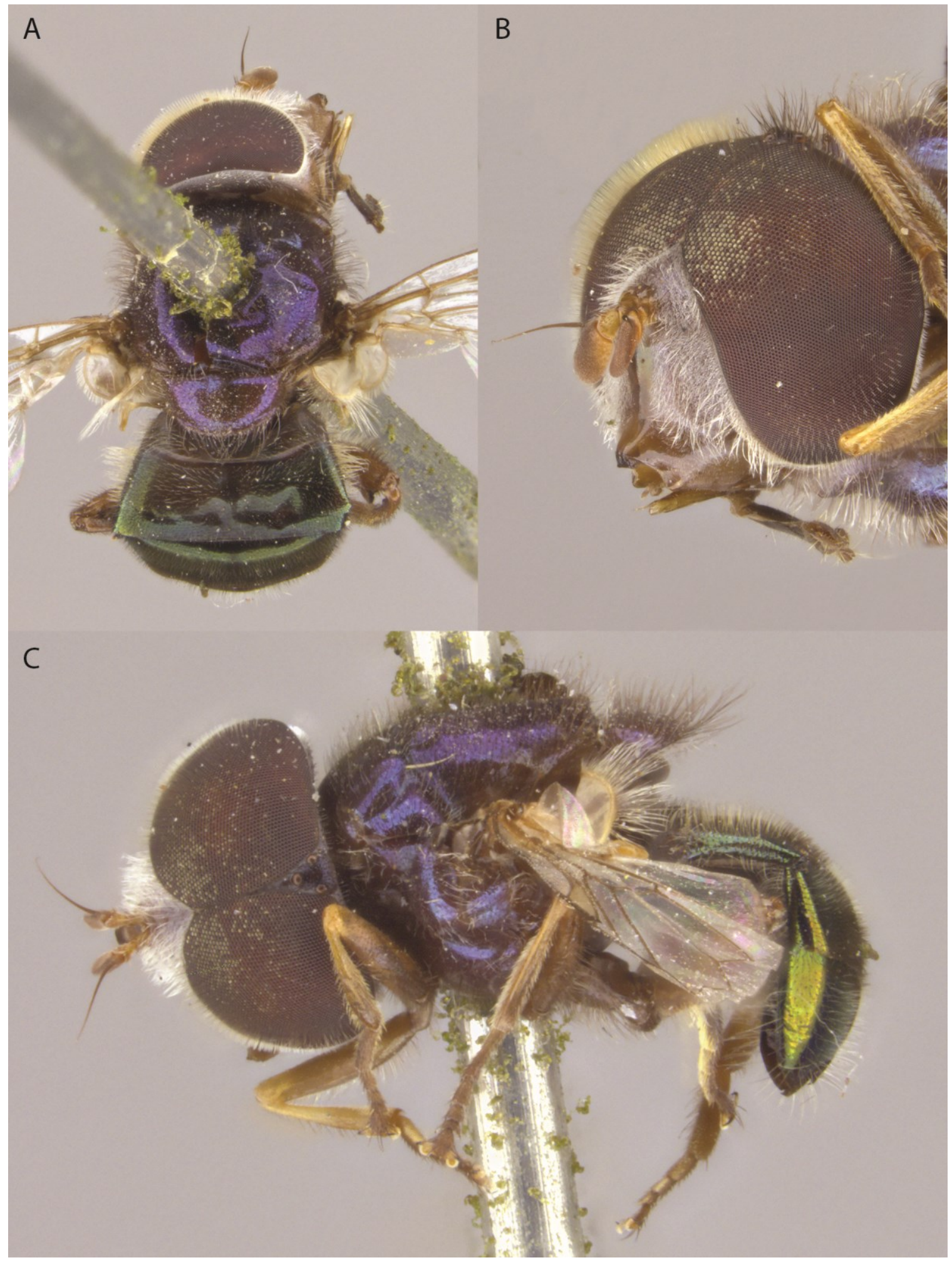

Chapter 3, Figure 28. P. pallidifacies. A) male dorsal habitus, B) male anterolateral face, C) male lateral habitus. 


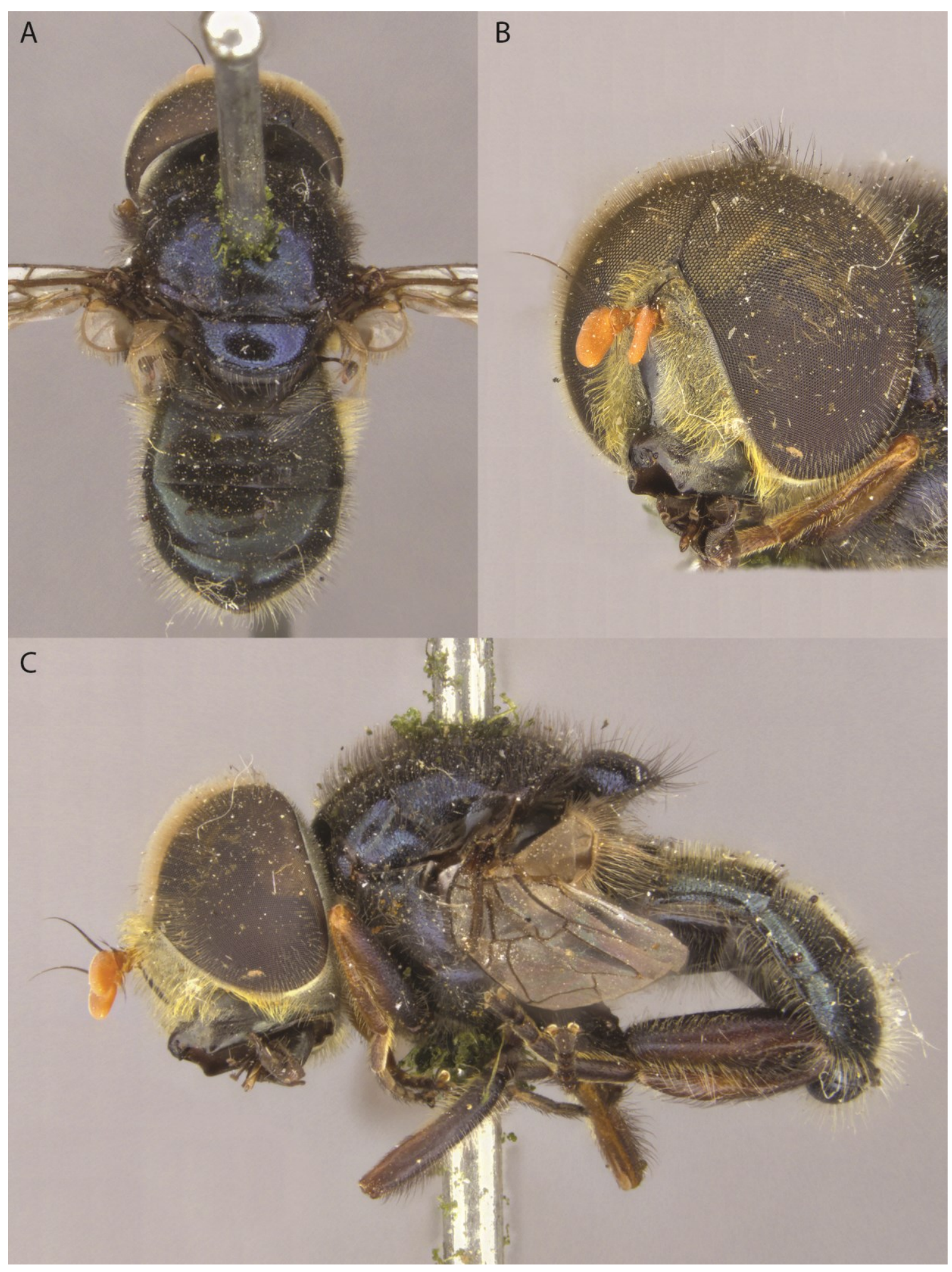

Chapter 3, Figure 29. P. pollinosa. A) male dorsal habitus, B) male anterolateral face, C) male lateral habitus. 


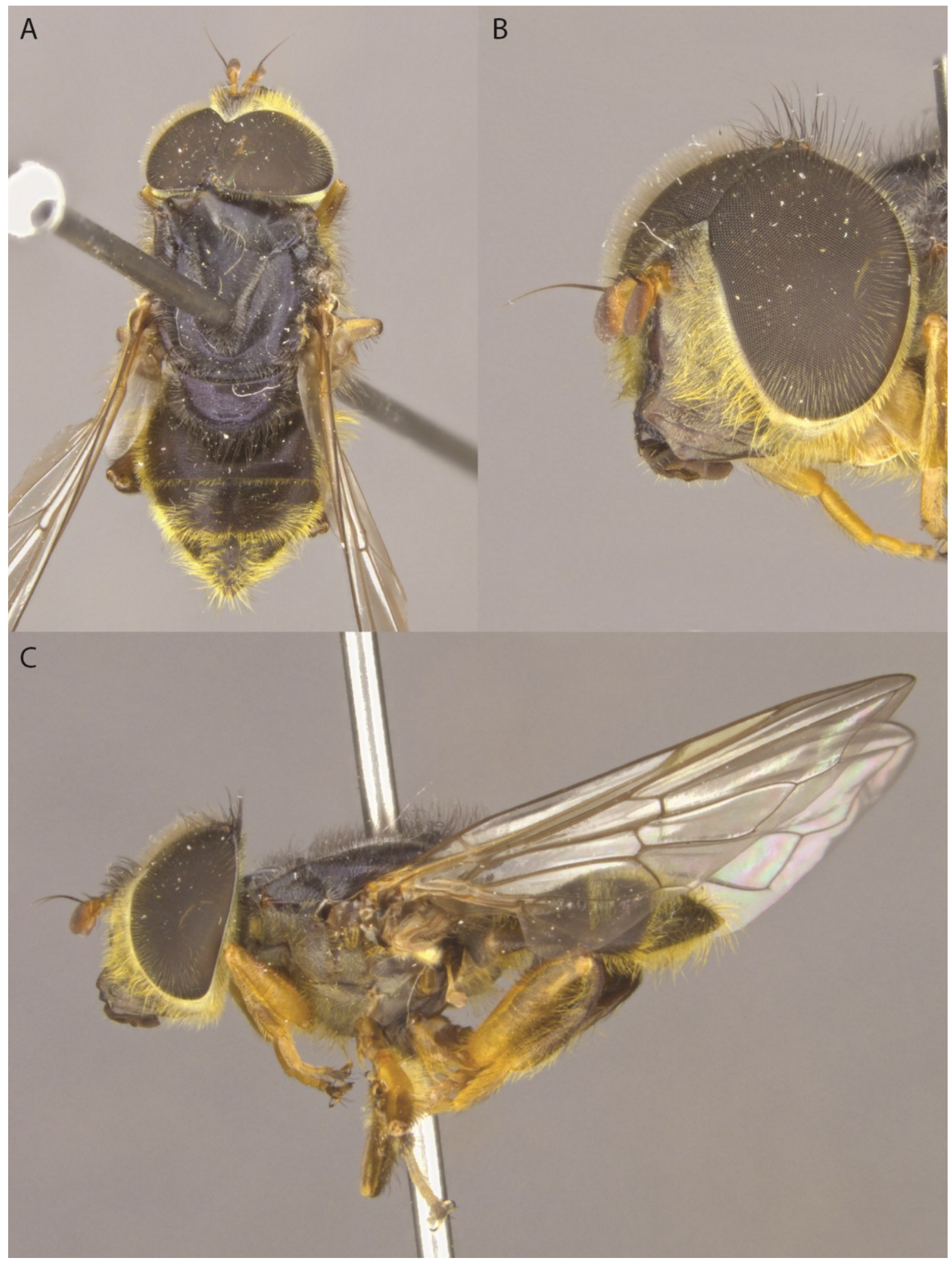

Chapter 3, Figure 30. P. solata. A) male dorsal habitus, B) male anterolateral face, C) male lateral habitus. 


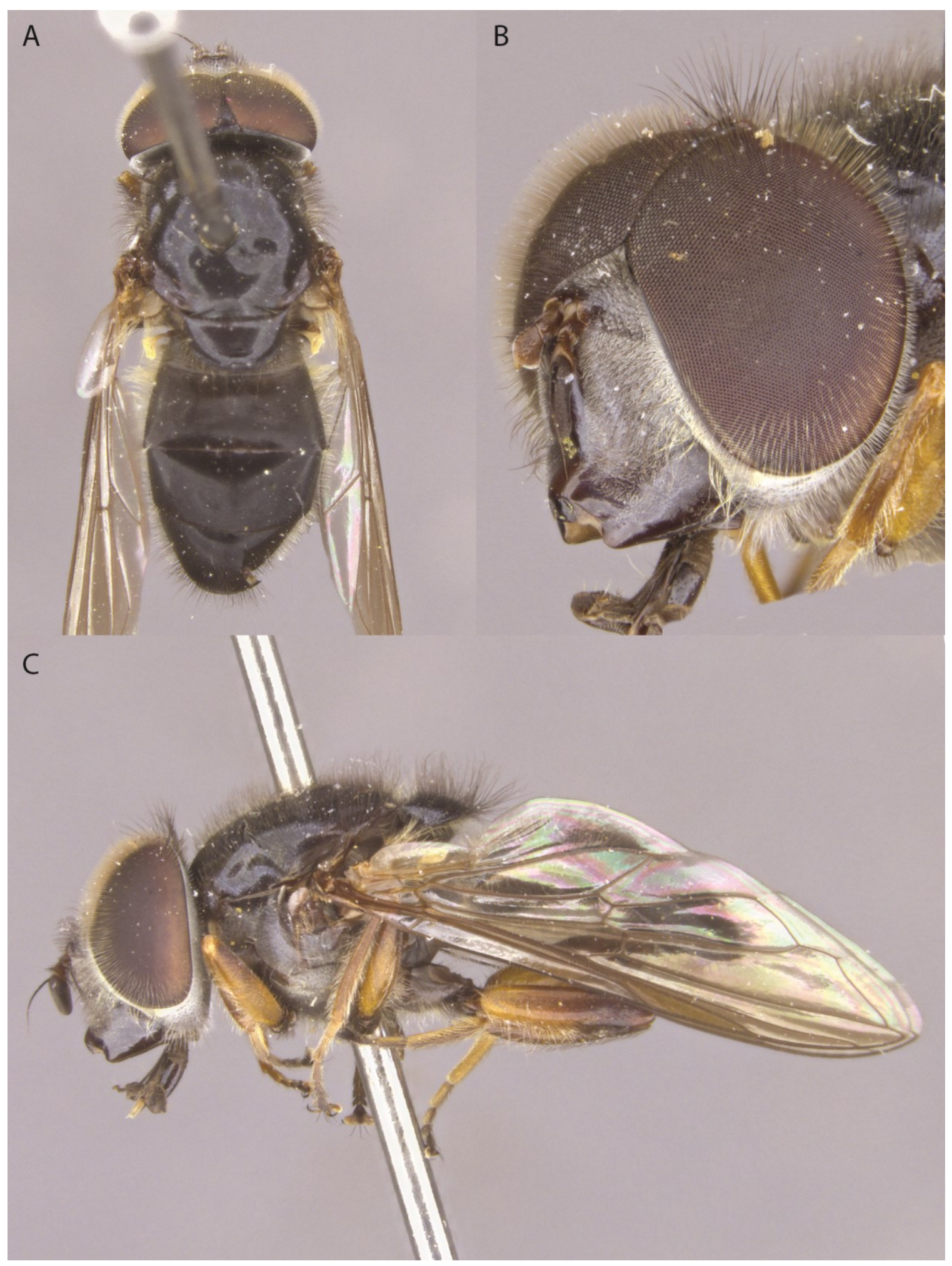

Chapter 3, Figure 31. P. spathistyla. A) male dorsal habitus, B) male anterolateral face, C) male lateral habitus. 


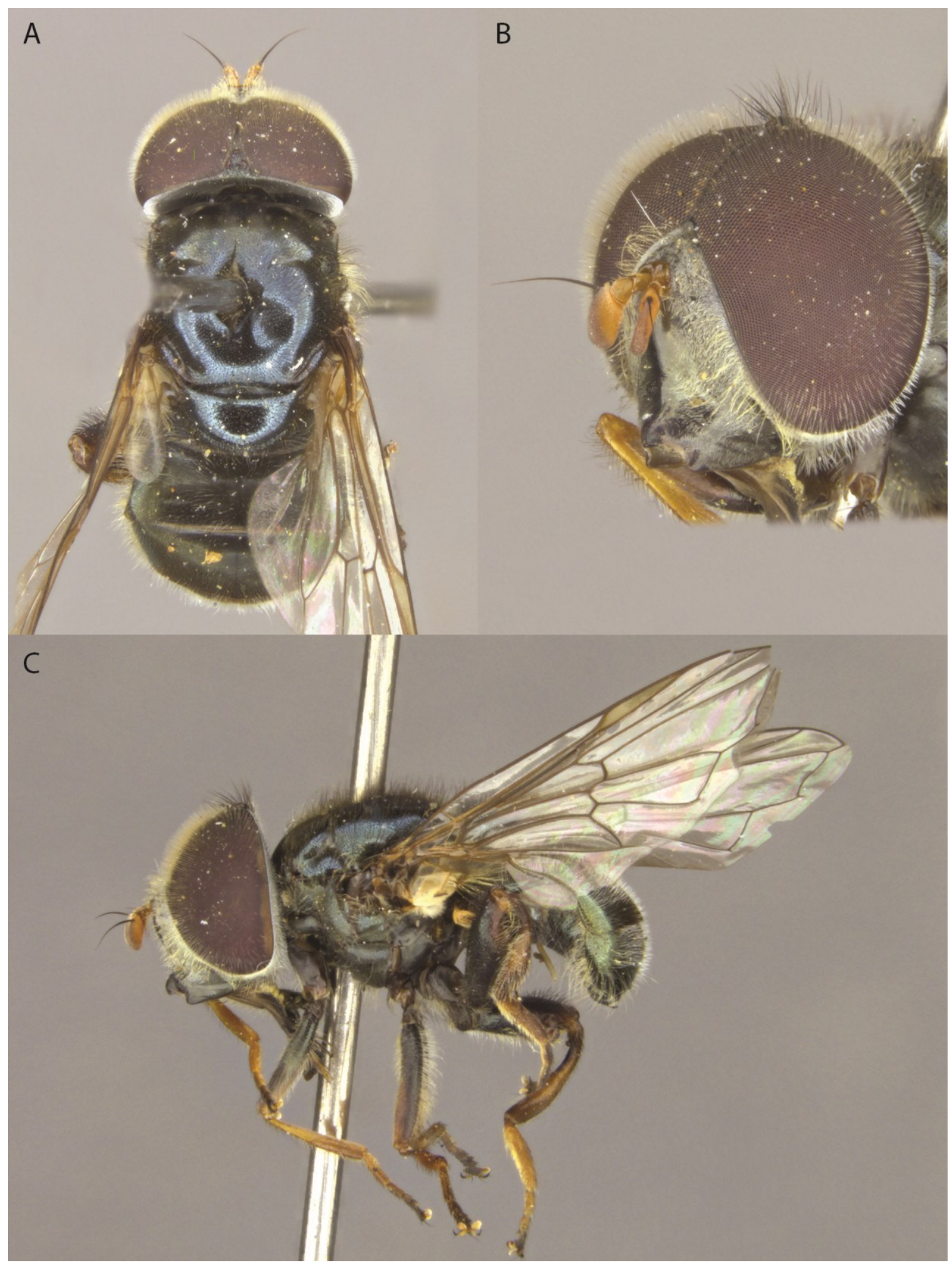

Chapter 3, Figure 32. P. spinifemur. A) male dorsal habitus, B) male anterolateral face, C) male lateral habitus. 


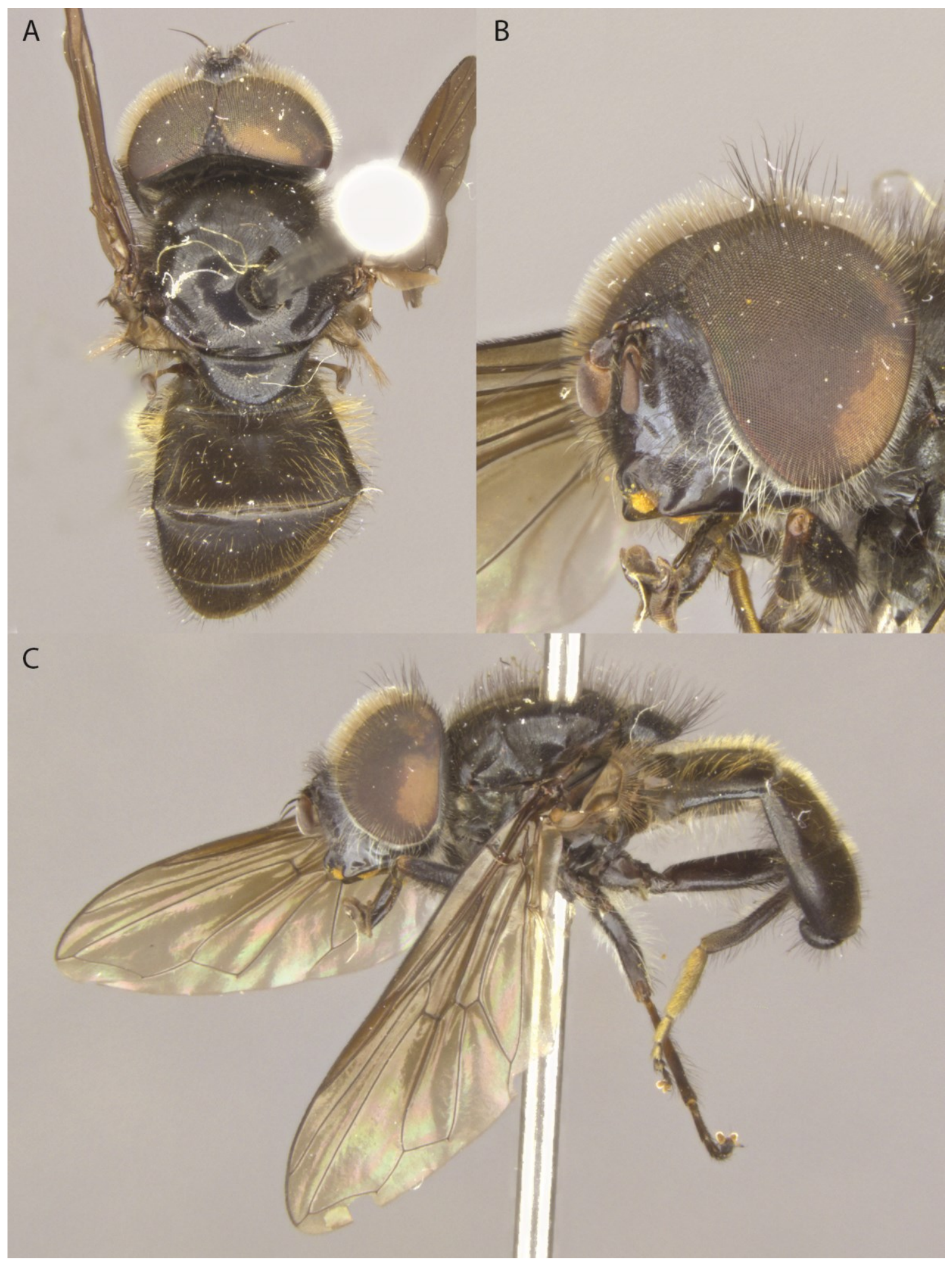

Chapter 3, Figure 33. P. tristis. A) male dorsal habitus, B) male anterolateral face, C) male lateral habitus. 


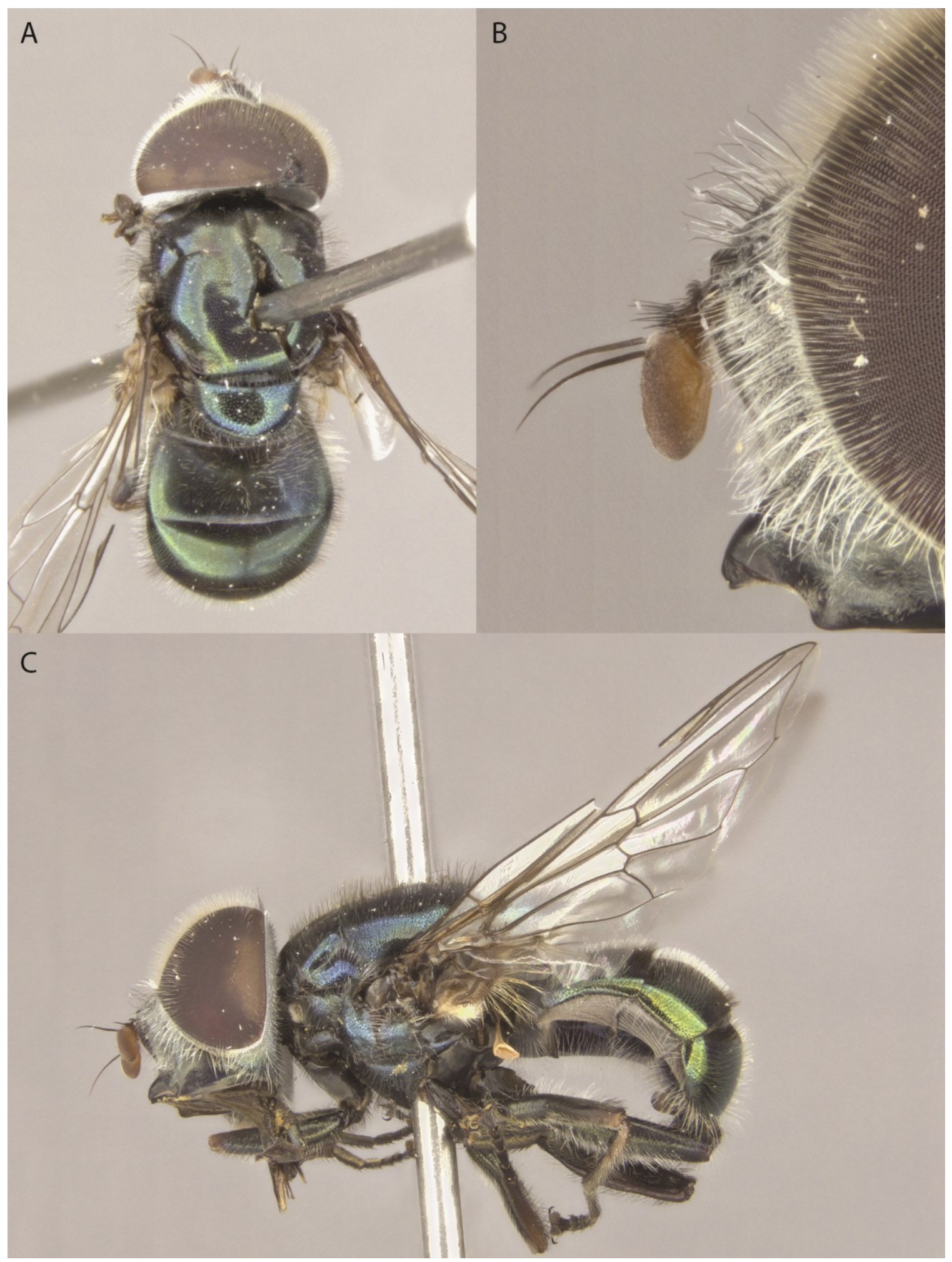

Chapter 3, Figure 34. P. viridis. A) male dorsal habitus, B) male anterolateral face, C) male lateral habitus. 


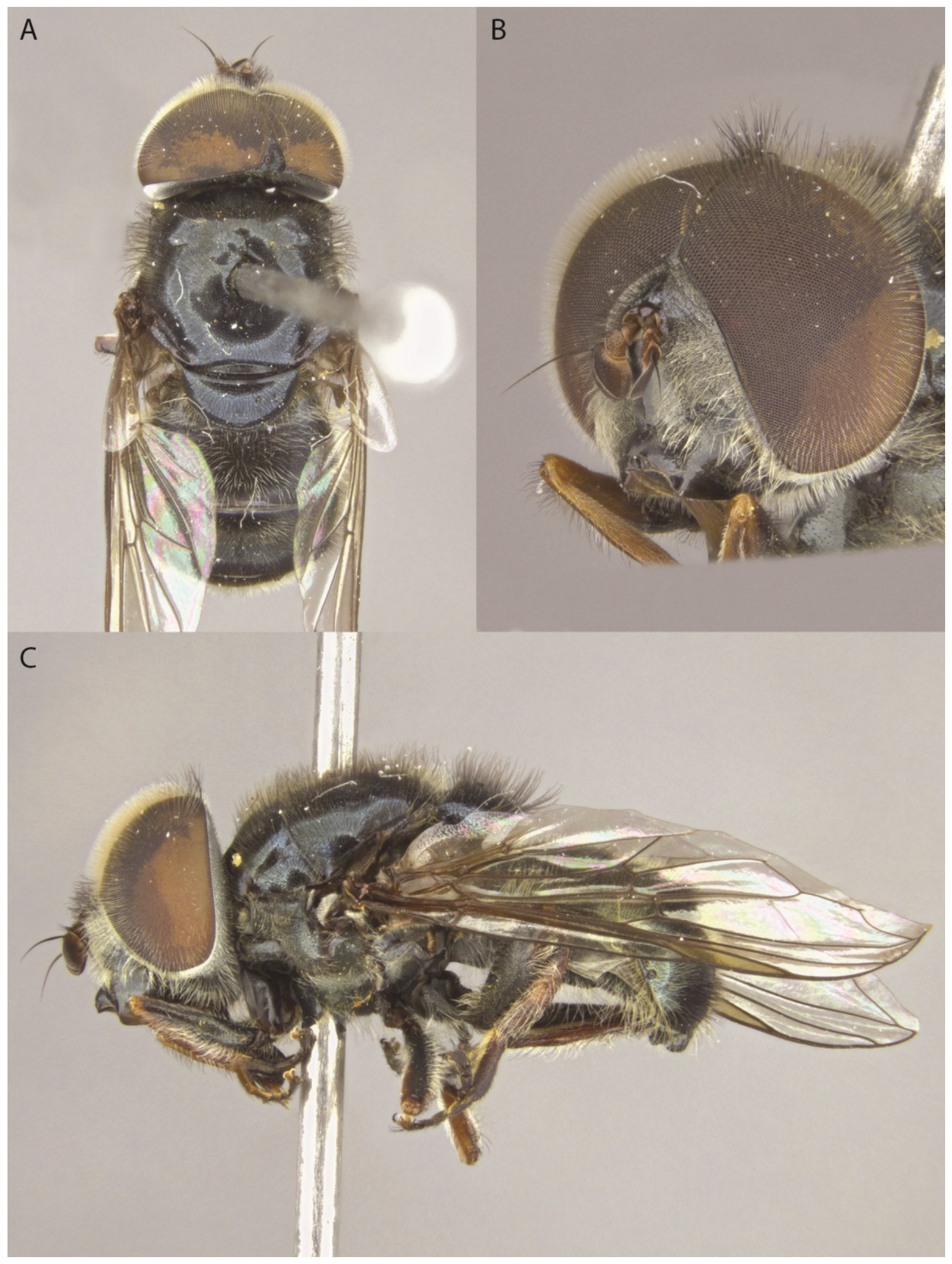

Chapter 3, Figure 35. P. zophos. A) male dorsal habitus, B) male anterolateral face, C) male lateral habitus. 
A

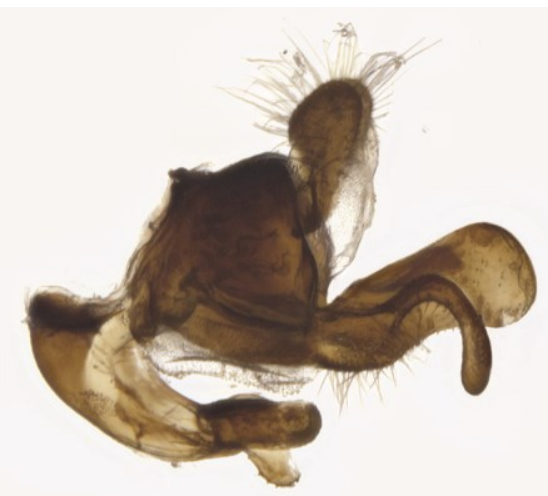

C

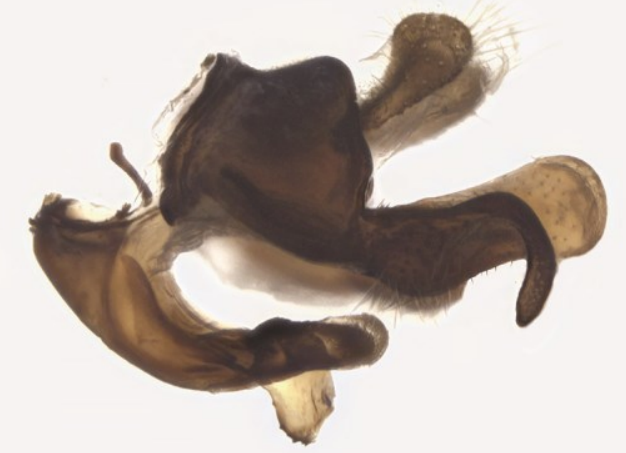

E

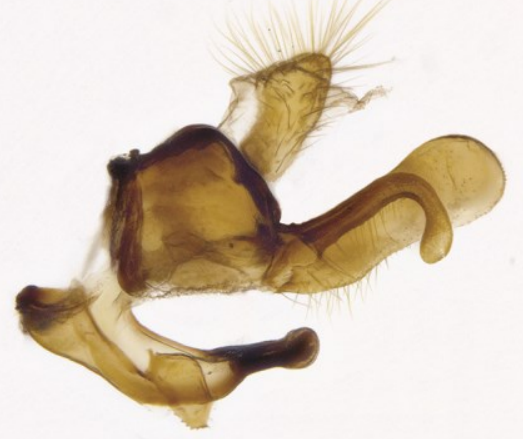

G

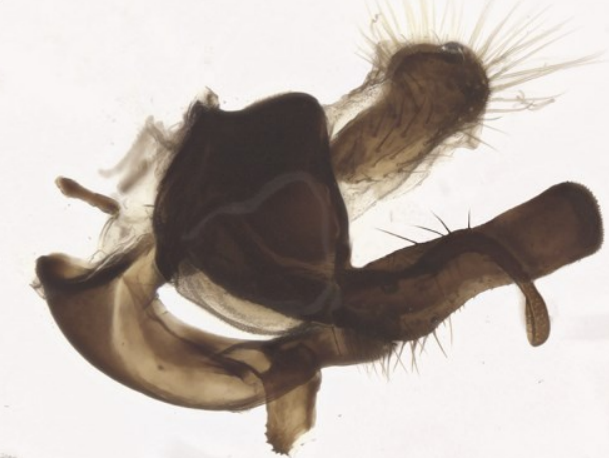

B

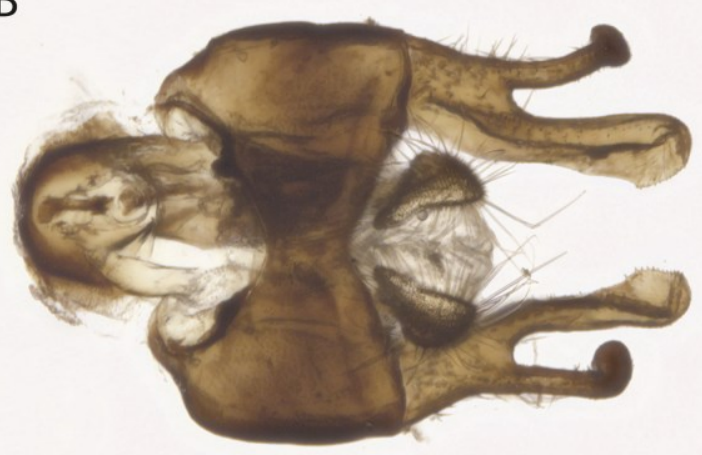

D

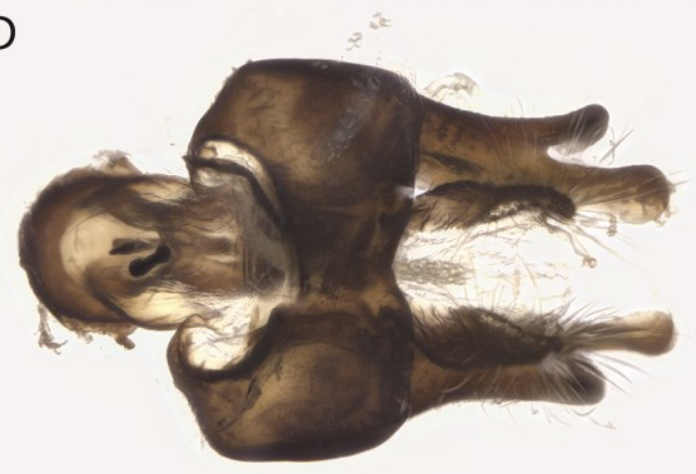

$\mathrm{F}$

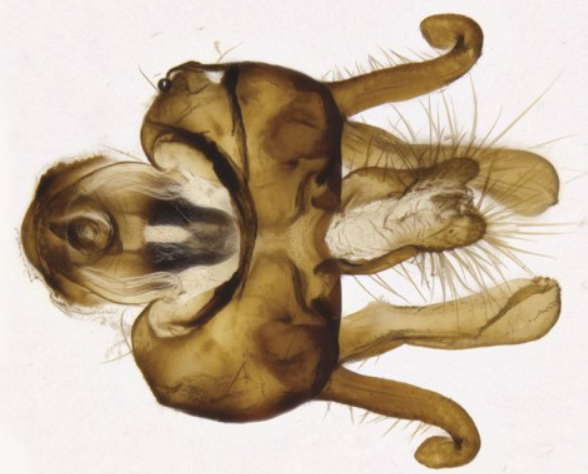

$\mathrm{H}$

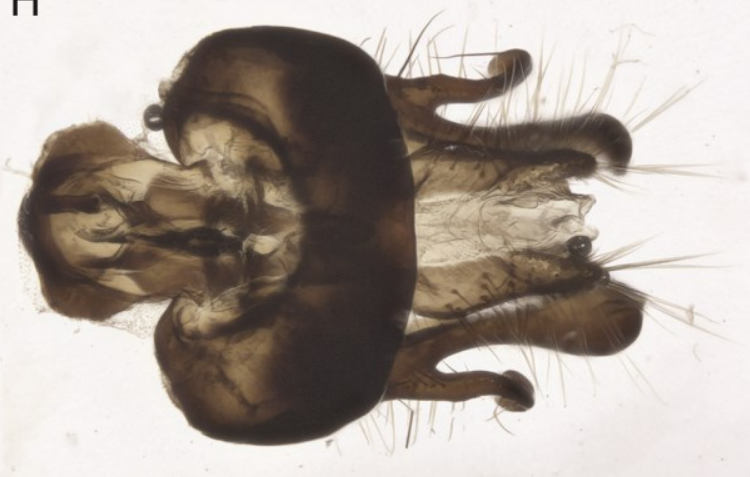

Chapter 3, Figure 36. Lateral and dorsal male genitalia of A, B) P. aislinni; C, D) $P$. alexanderi; E, F) P. apiformis, and G, H) P. auricauda. 
A

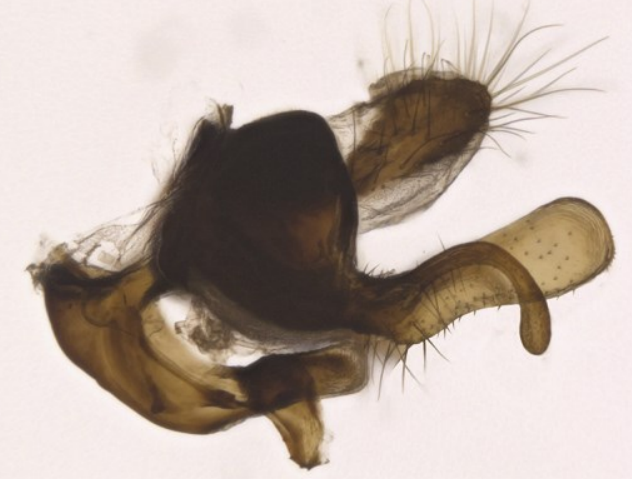

C

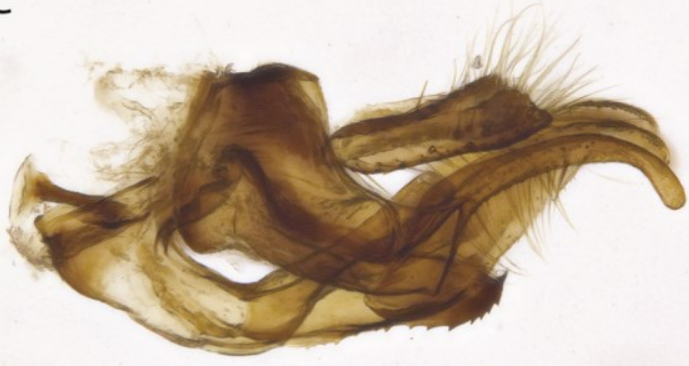

E

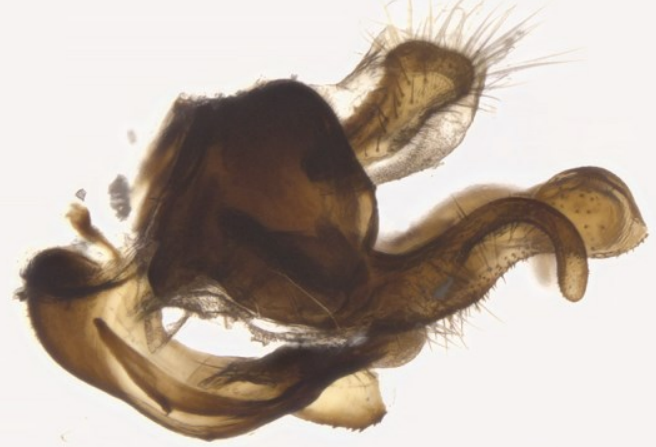

G

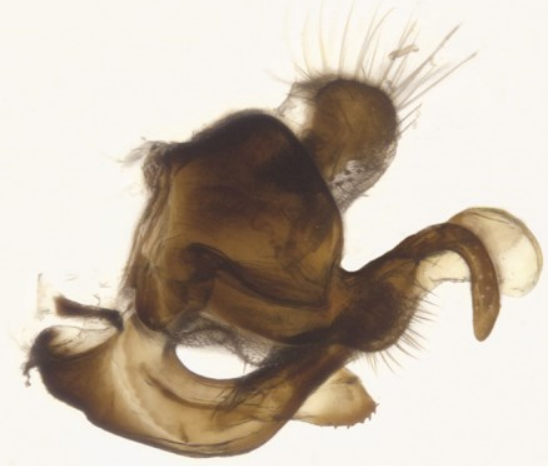

B

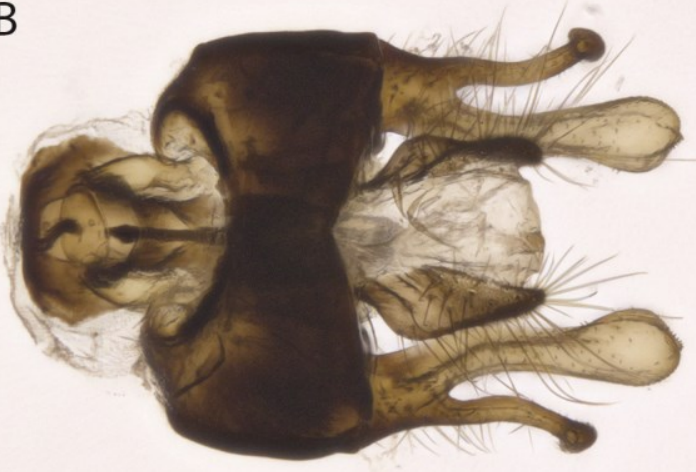

D

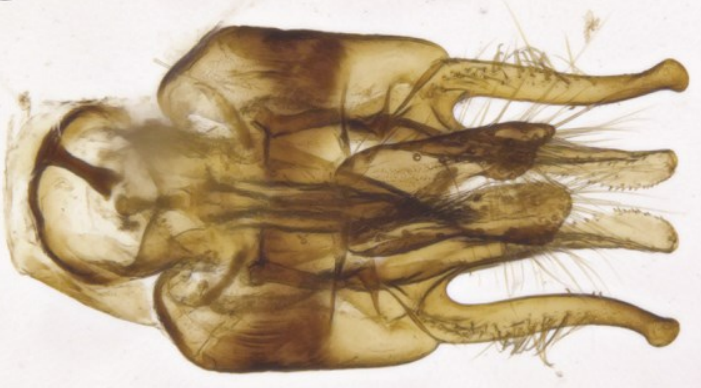

$\mathrm{F}$

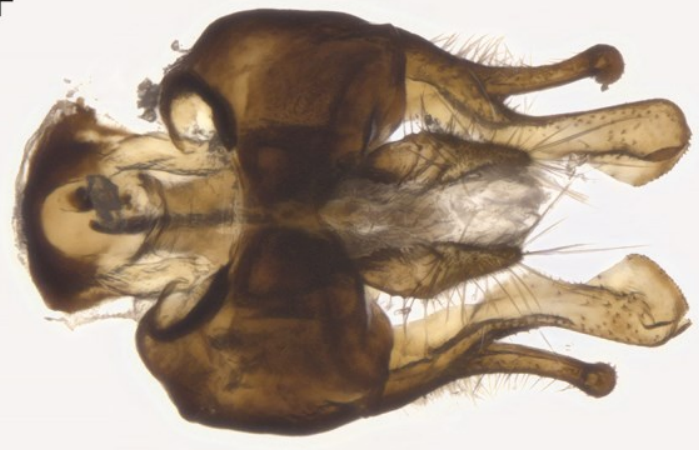

$\mathrm{H}$

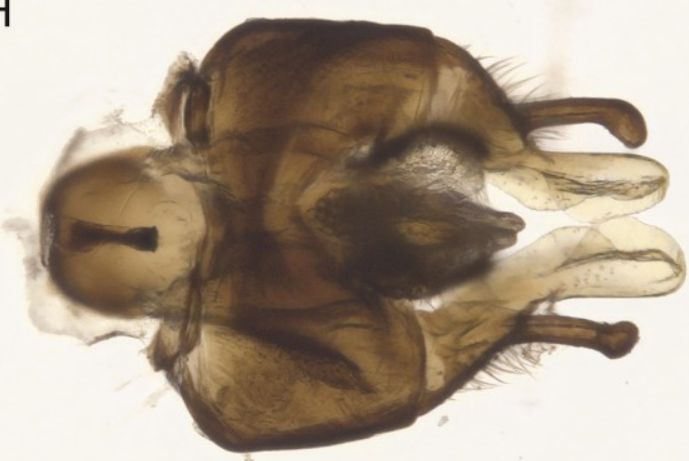

Chapter 3, Figure 37. Lateral and dorsal male genitalia of A, B) P. auripilosa; C, D) P. austropsilota; E, F) P. azurea, and G, H) P. basalis. 
A

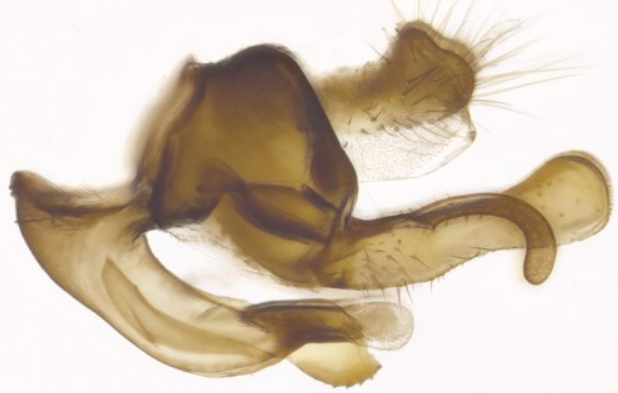

C

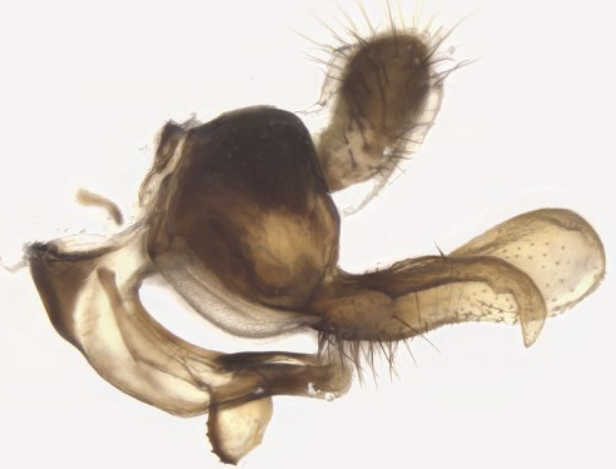

$\mathrm{E}$

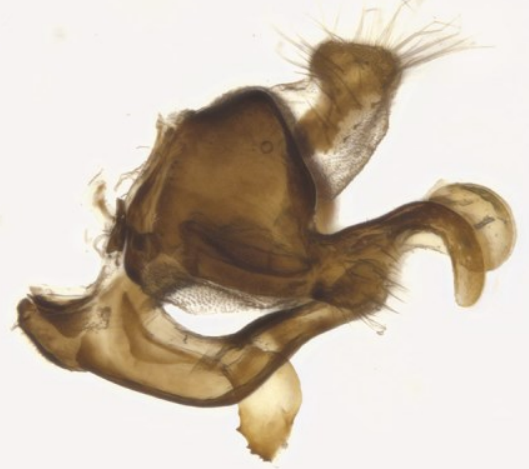

G

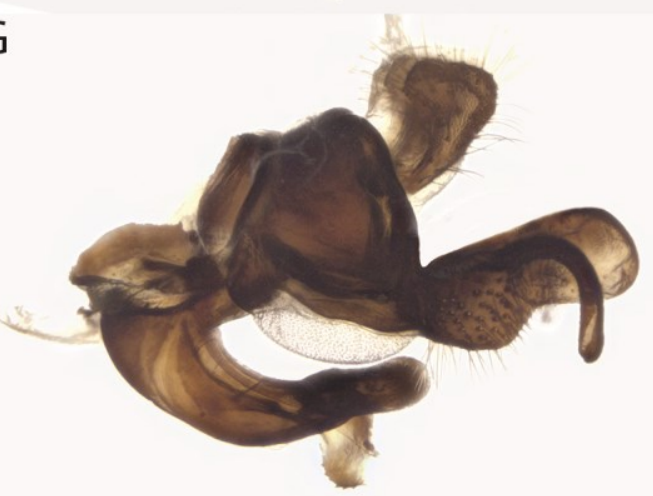

B

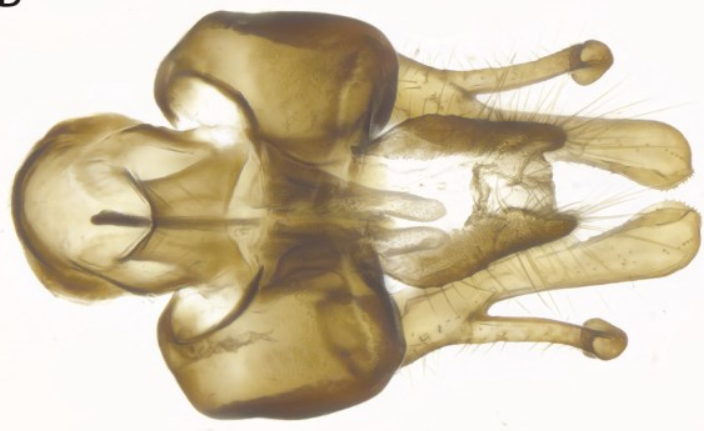

D

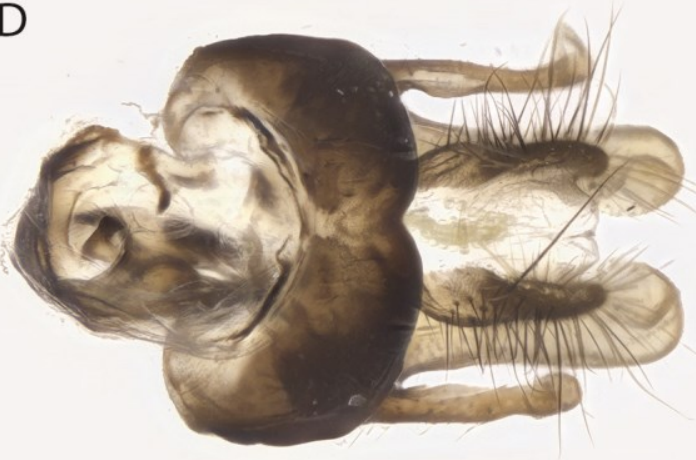

$\mathrm{F}$

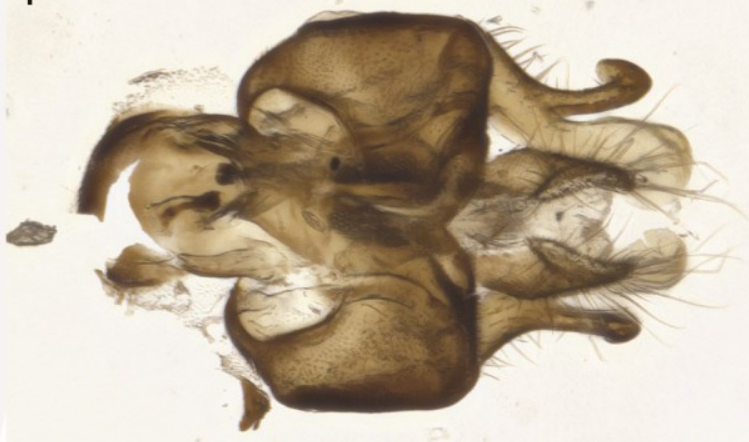

$\mathrm{H}$

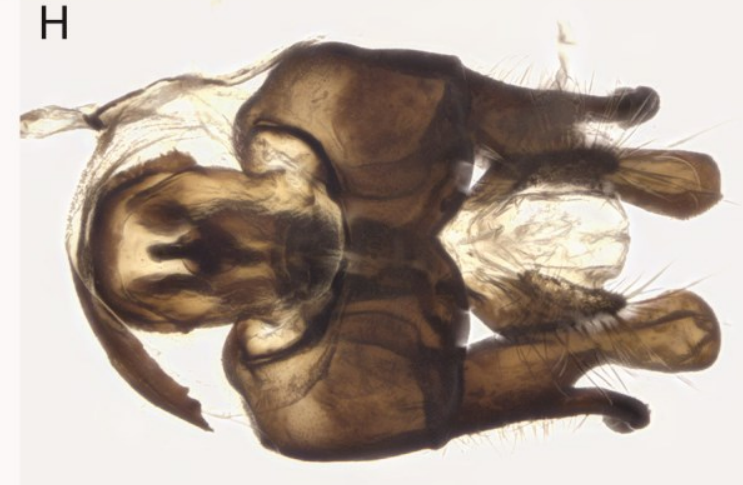

Chapter 3, Figure 38. Lateral and dorsal male genitalia of A, B) P. bicolor; C, D) $P$. brunnipennis; E, F) P. calva, and G, H) P. coerulea. 
A

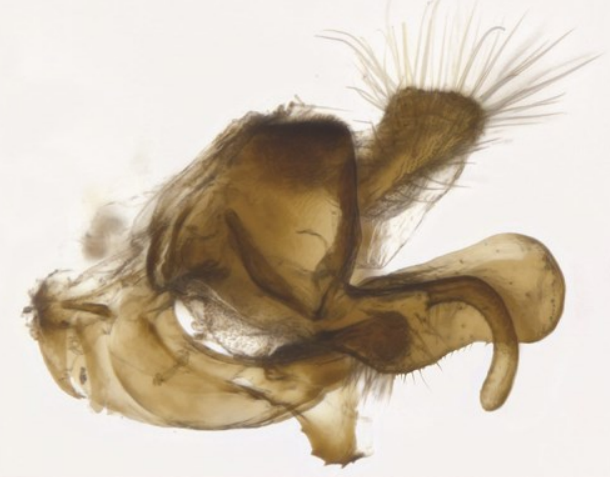

C

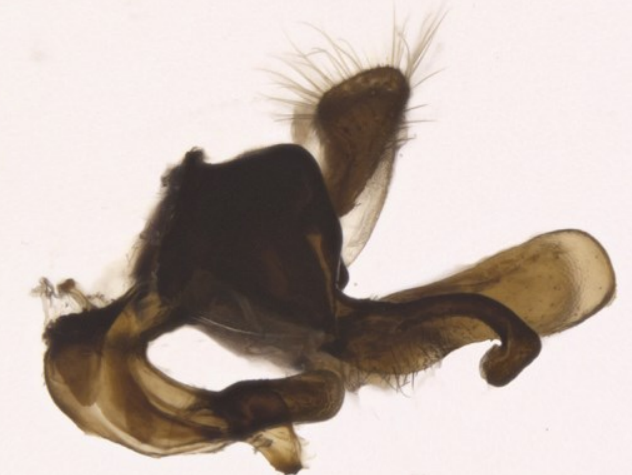

$\mathrm{E}$

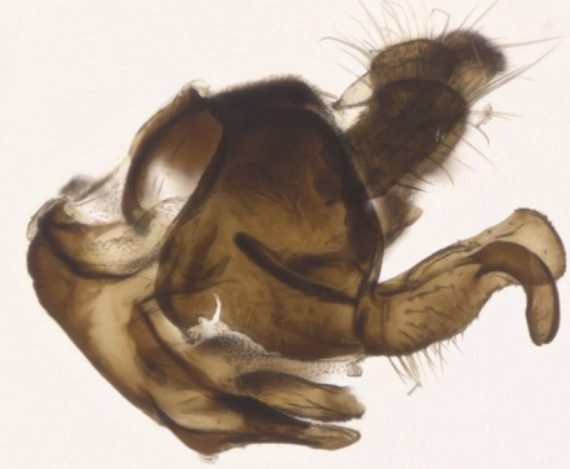

G

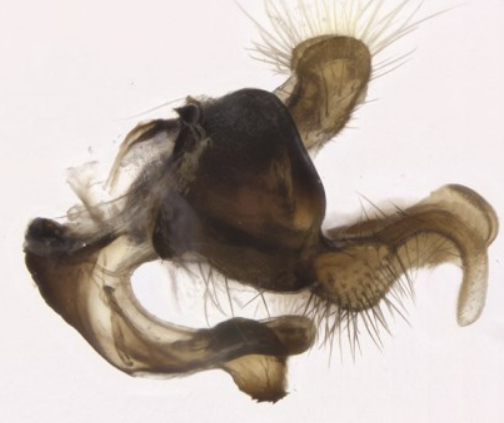

B

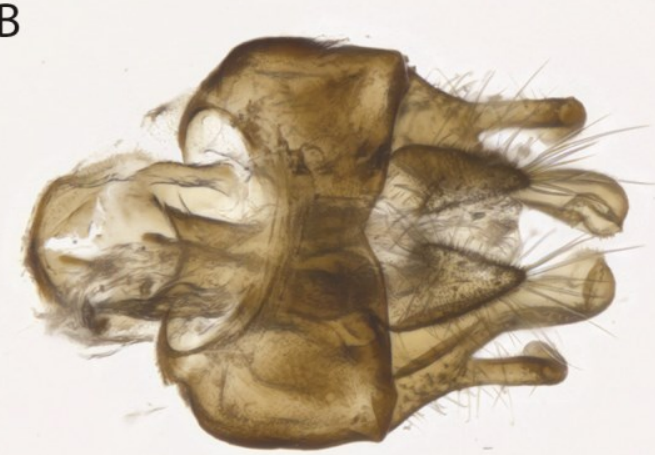

D

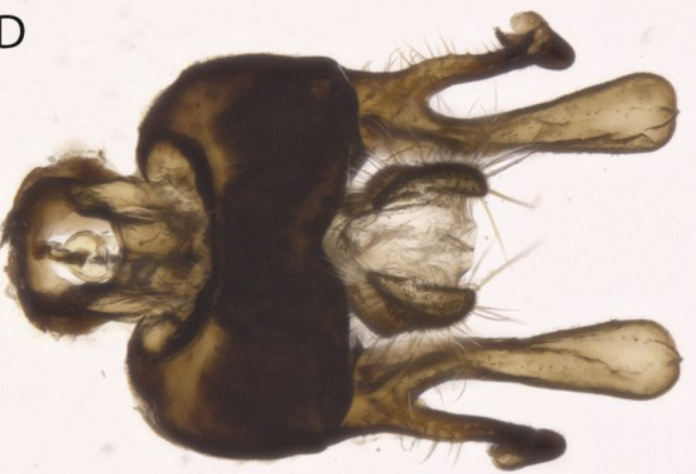

$\mathrm{F}$

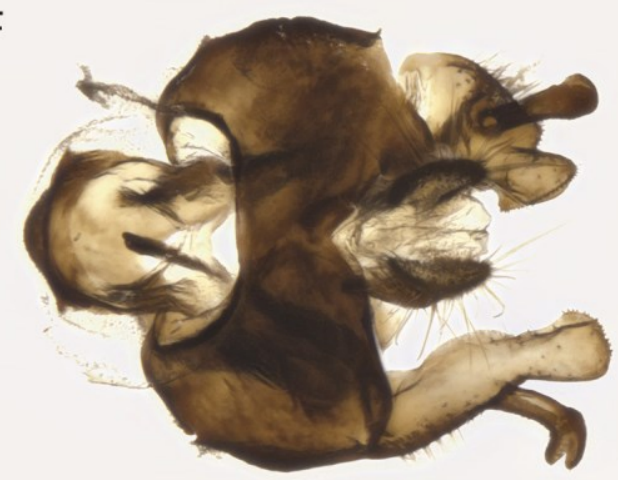

$\mathrm{H}$

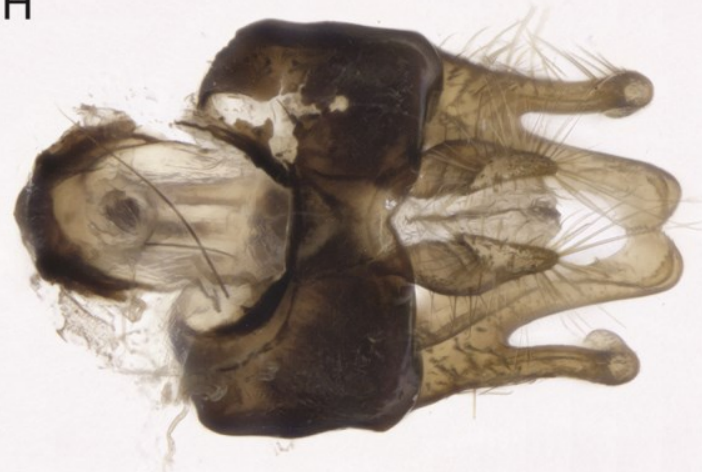

Chapter 3, Figure 39. Lateral and dorsal male genitalia of A, B) P. darwini; C, D) $P$. femoralis; E, F) P. lividus, and G, H) P. longipilosa. 
A

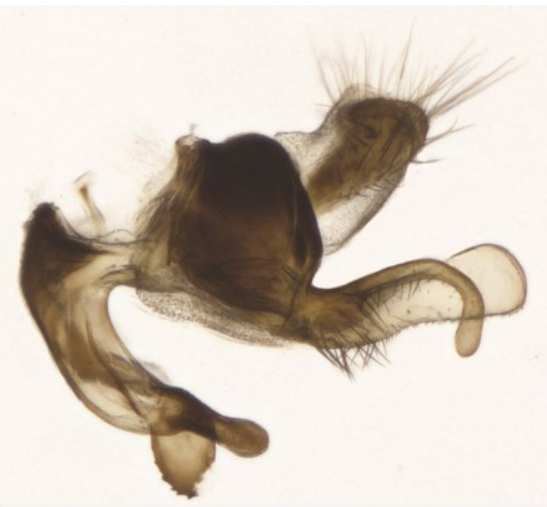

C

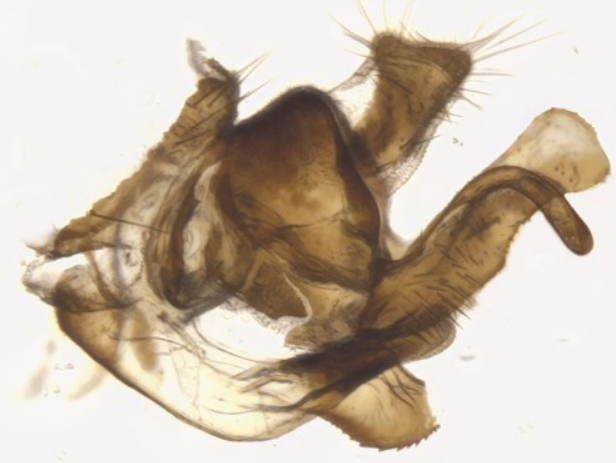

$E$

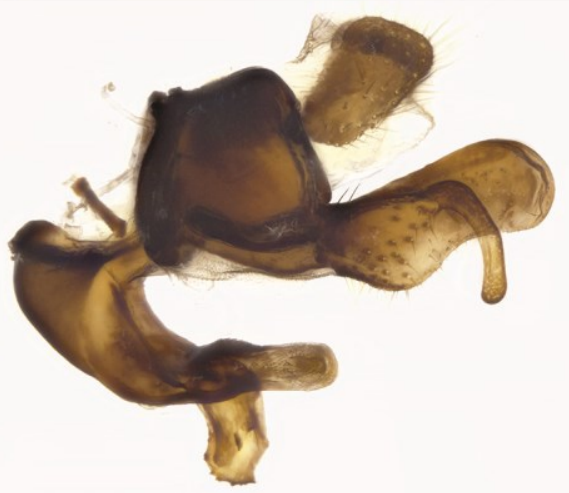

G

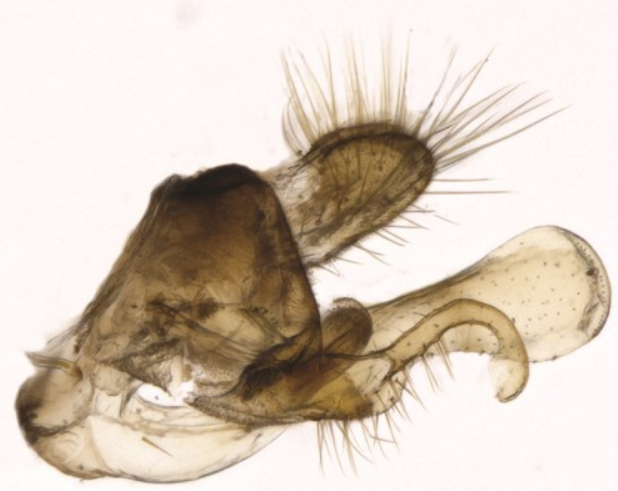

B
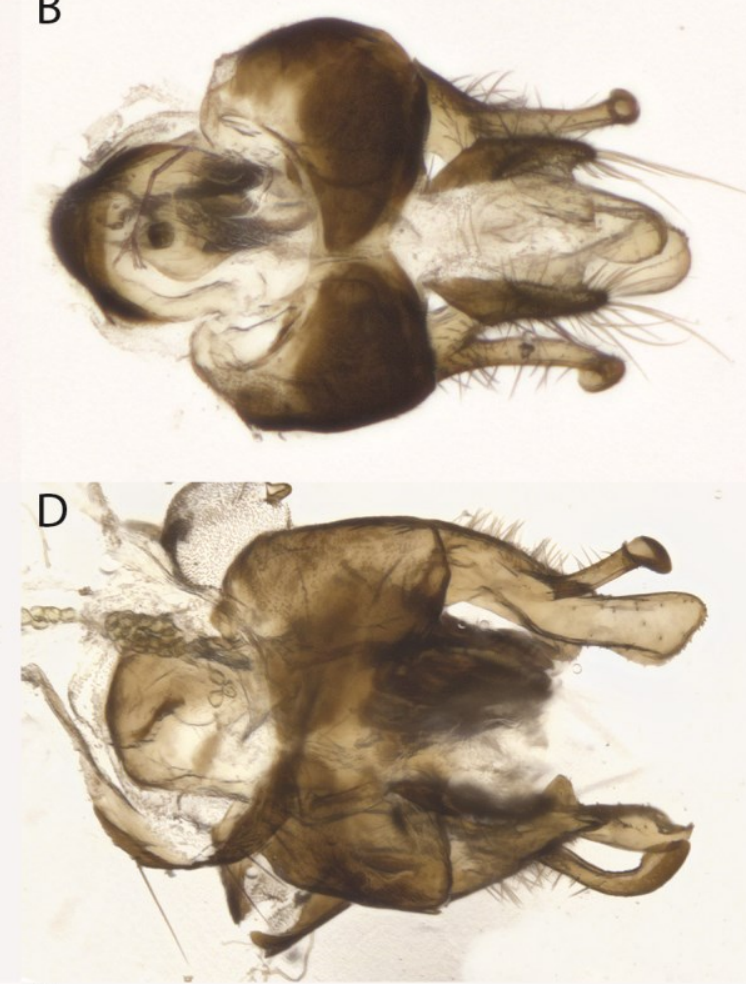

$\mathrm{F}$

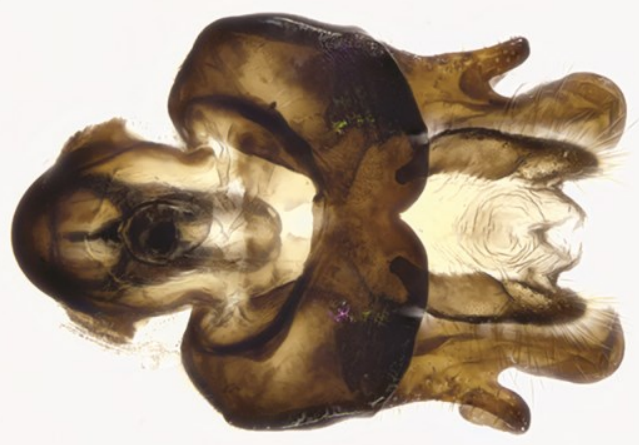

$\mathrm{H}$

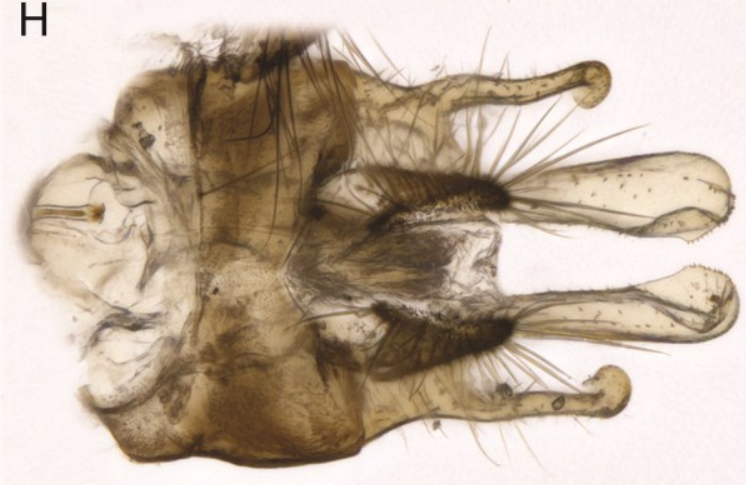

Chapter 3, Figure 40. Lateral and dorsal male genitalia of A, B) P. maculata; C, D) $P$. mcqueeni; E, F) P. metallica, and G, H) P. nigripilosa. 
A

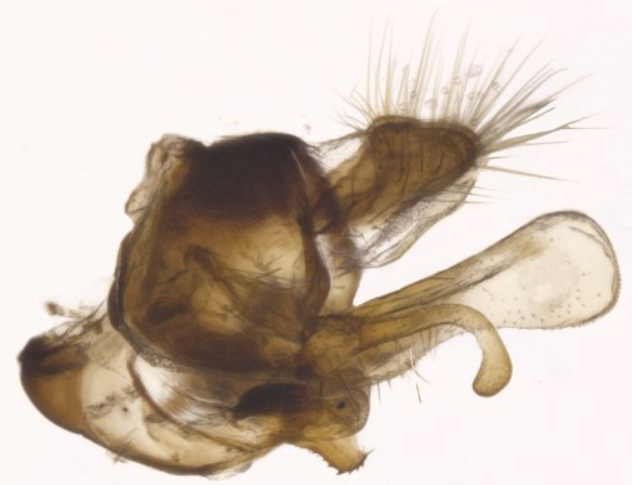

C

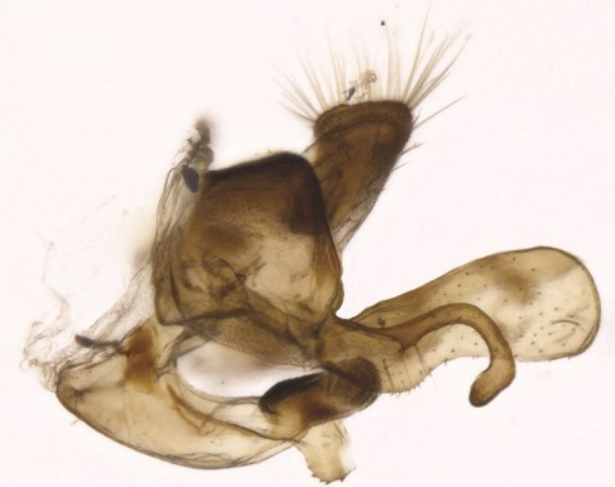

$E$

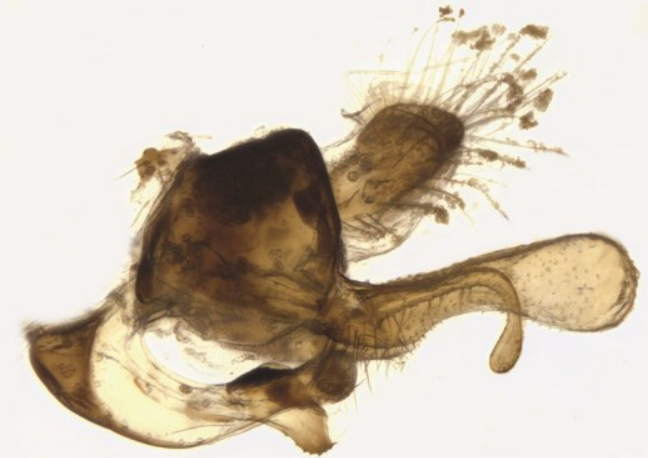

G

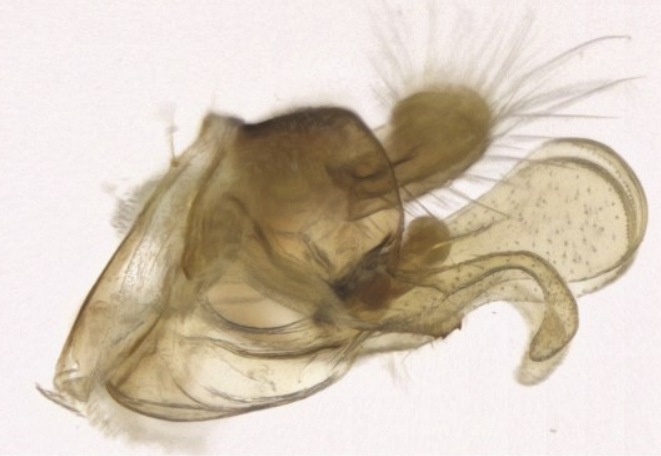

B

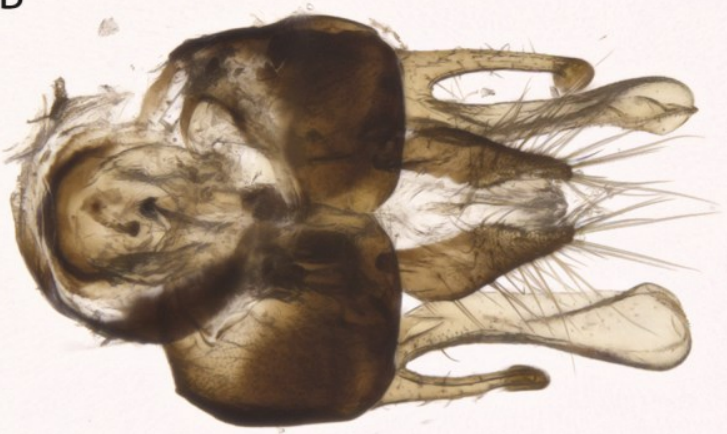

D
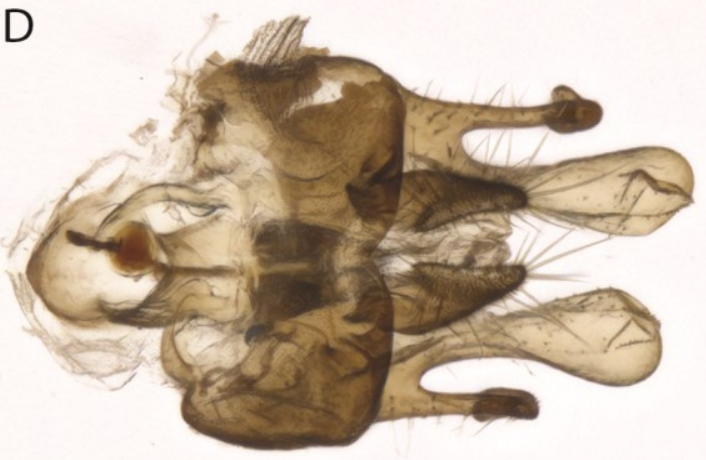

$\mathrm{F}$

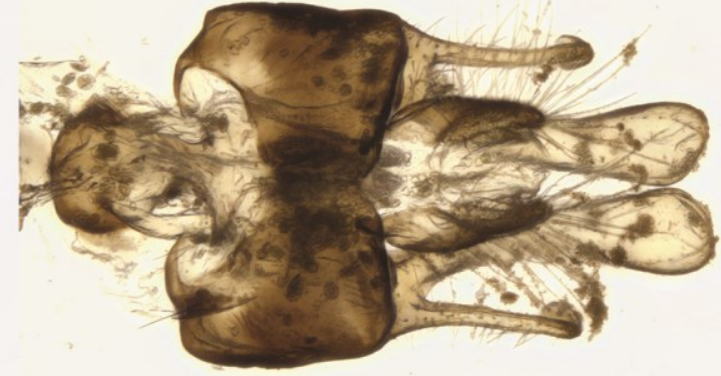

$\mathrm{H}$

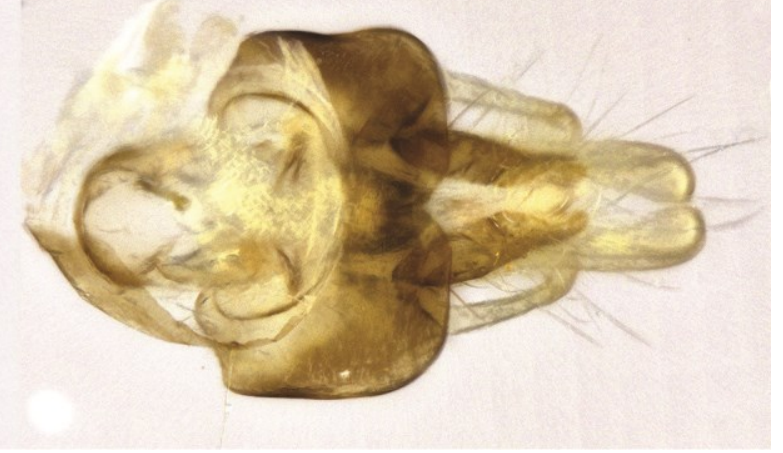

Chapter 3, Figure 41. Lateral and dorsal male genitalia of A, B) P. nitida; C, D) $P$. pallidifacies; E, F) P. pollinosa, and G, H) P. solata. 
A

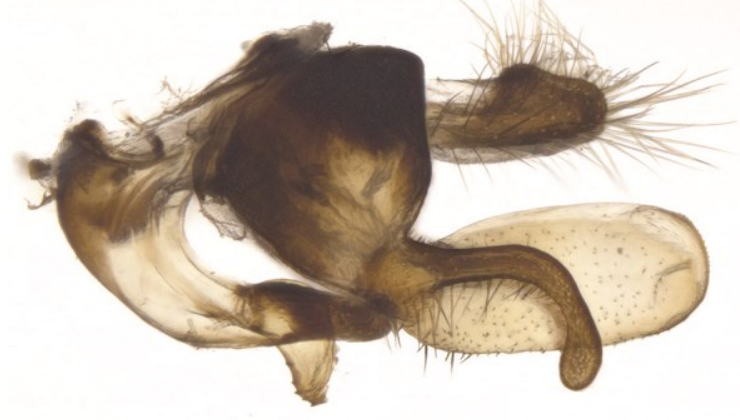

C

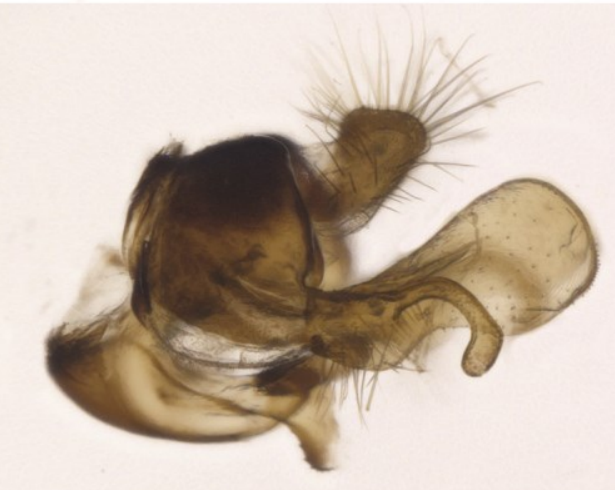

E

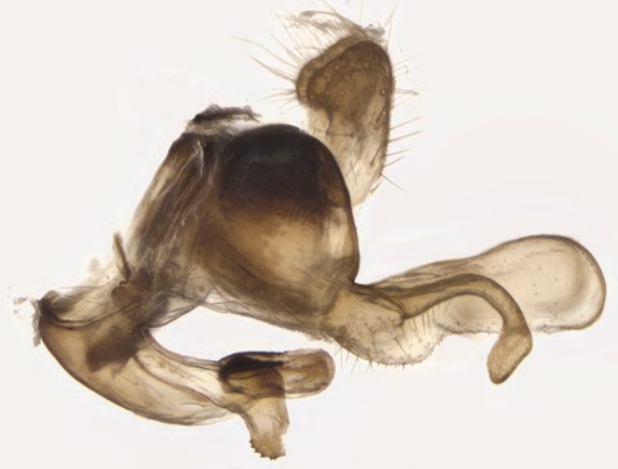

G

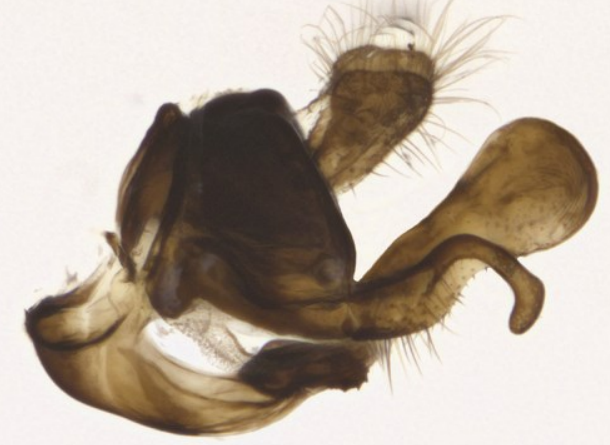

B

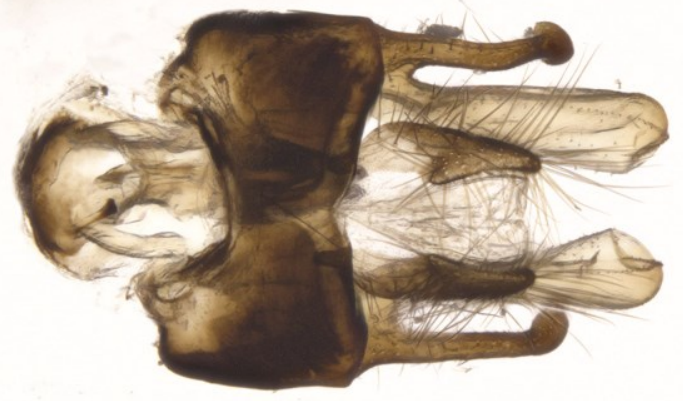

D

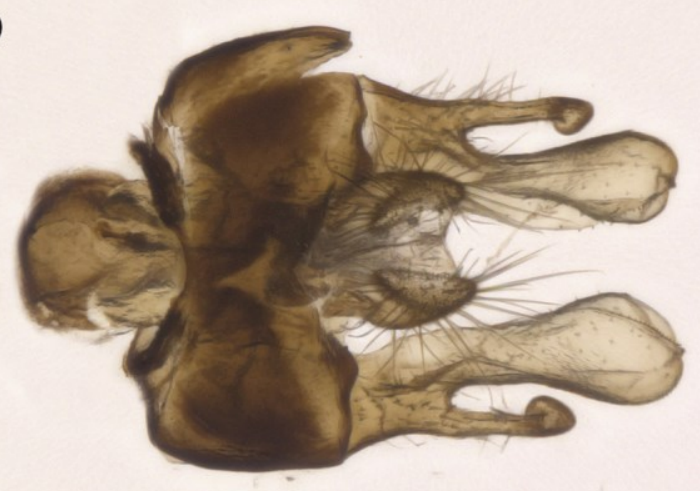

$\mathrm{F}$

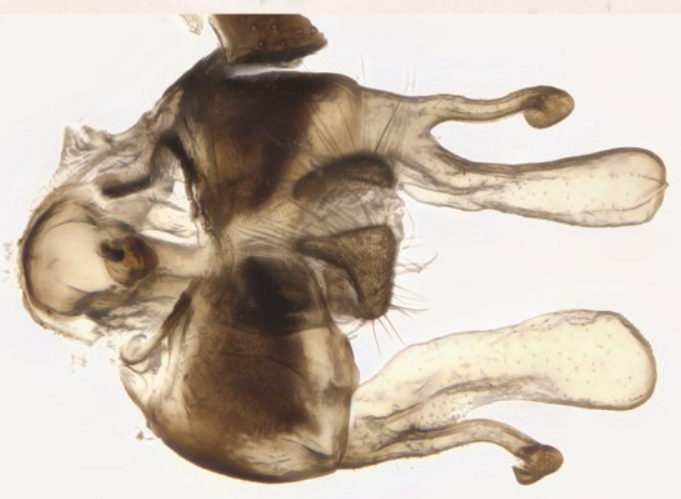

$\mathrm{H}$

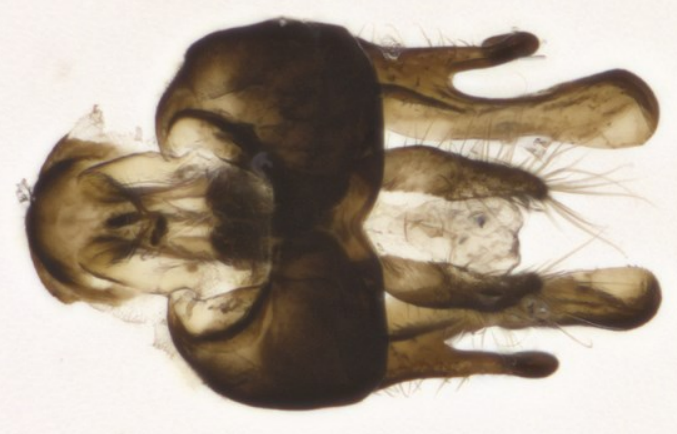

Chapter 3, Figure 42. Lateral and dorsal male genitalia of A, B) P. spathistyla; C, D) $P$. spinifemur; E, F) P. viridis; and G, H) P. zophos. 


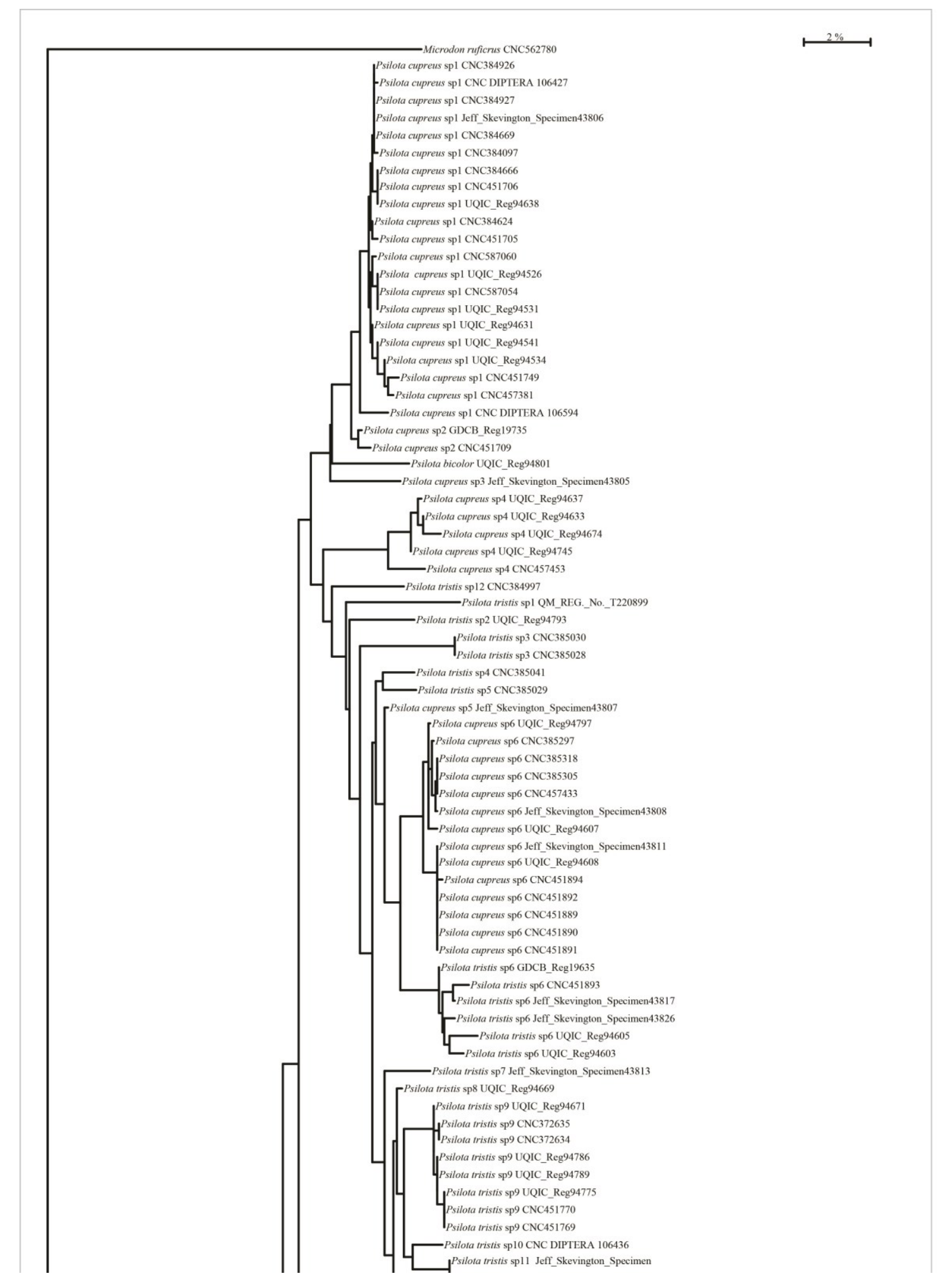

Chapter 3, Figure 43. Neighbor-joining tree of all available Australian Psilota DNA Barcodes. 


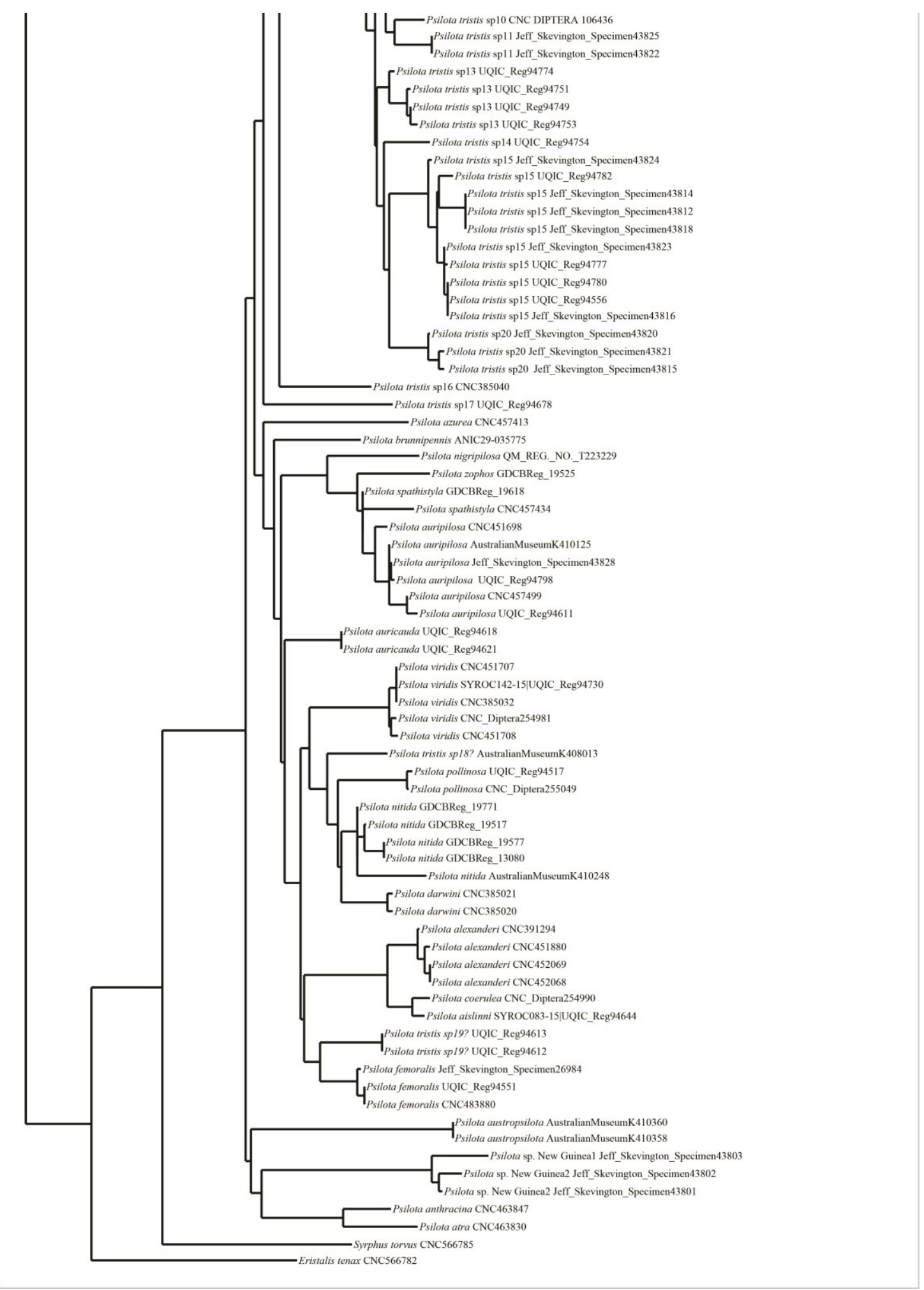

Figure 43 (conc'l). Neighbor-joining tree of all available Australian Psilota DNA Barcodes. 

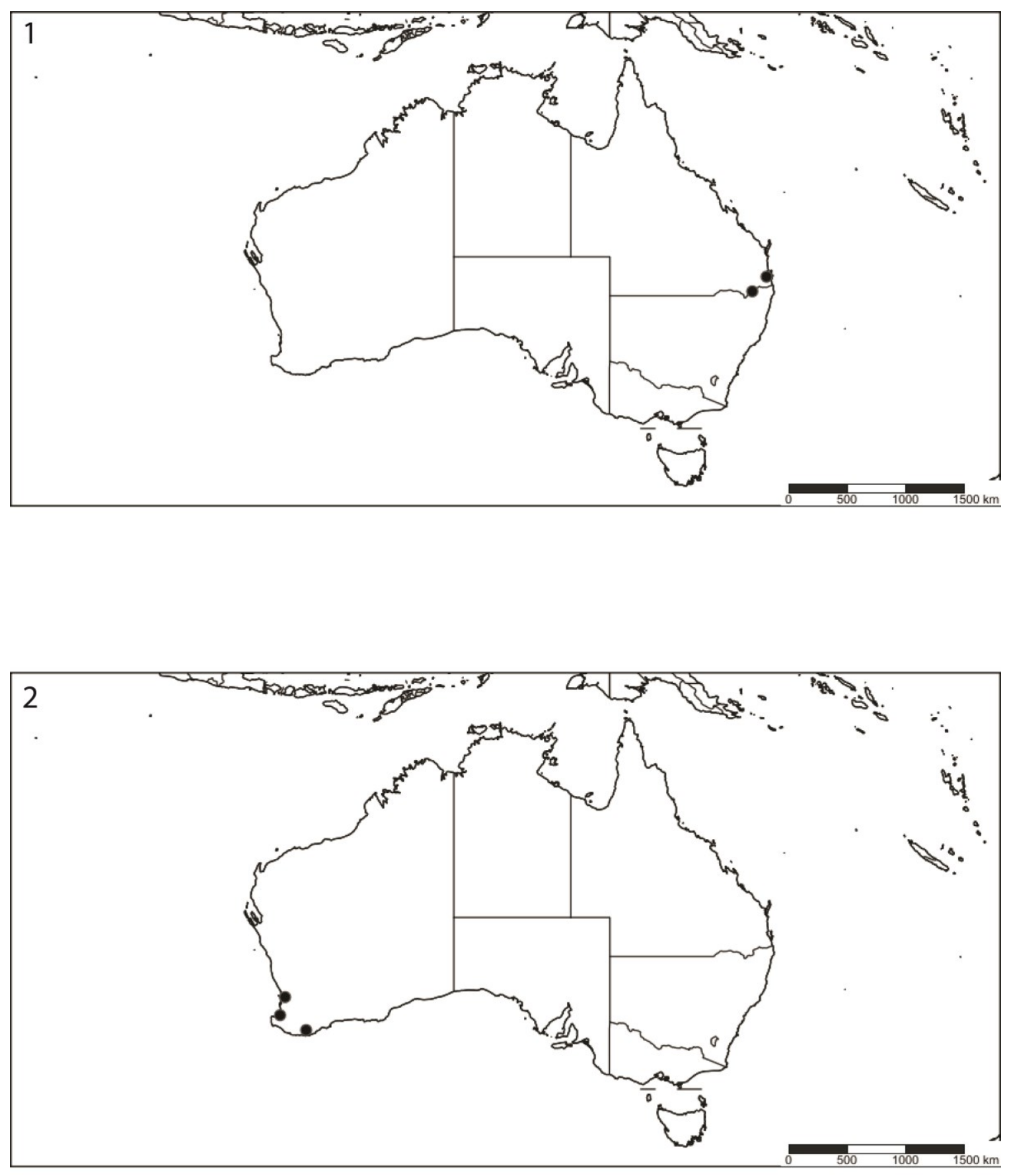

Chapter 3, Map 1: Psilota aislinni

Chapter 3, Map 2: Psilota alexanderi 

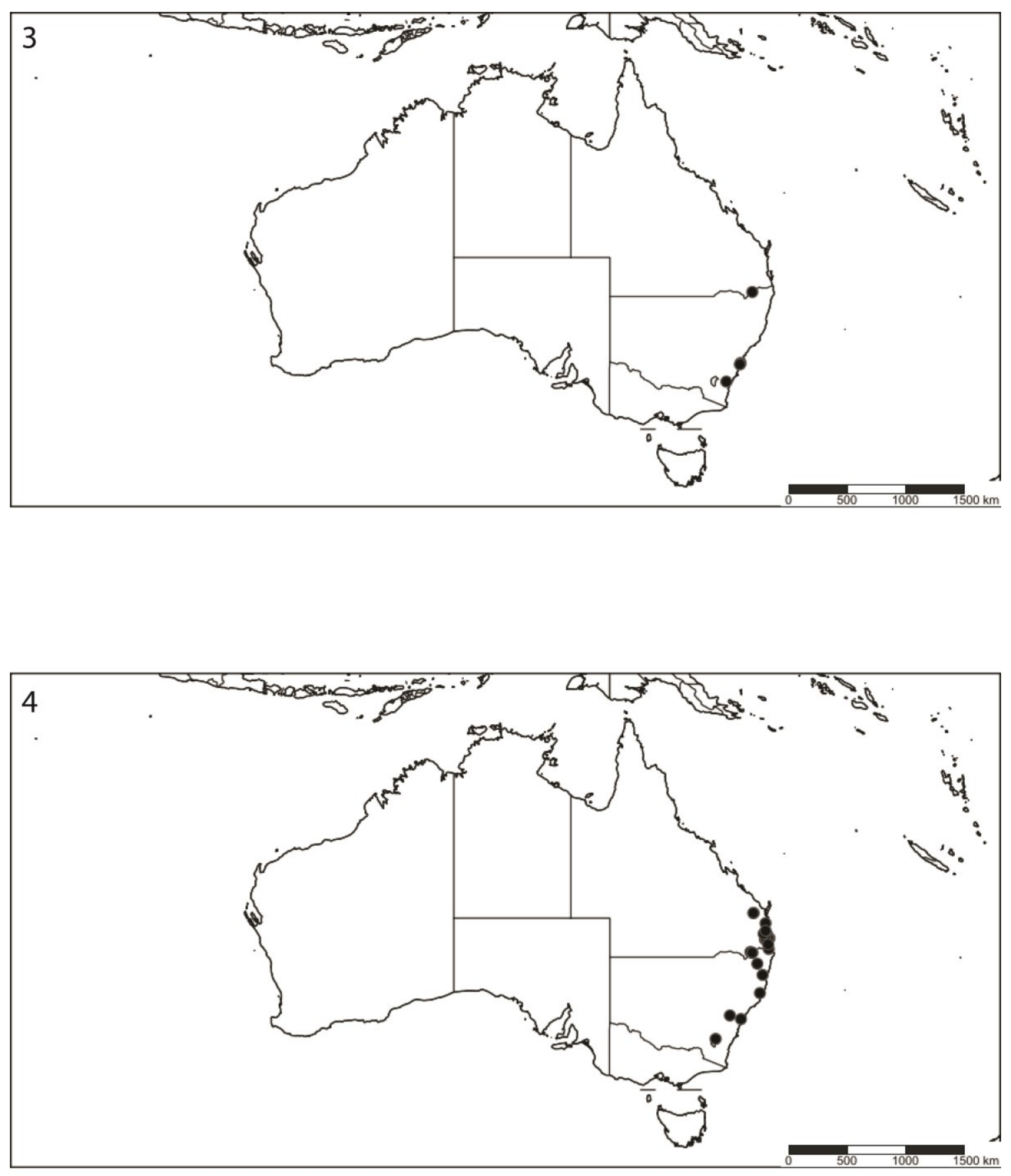

Chapter 3, Map 3: Psilota apiformis

Chapter 3, Map 4: Psilota auricauda 

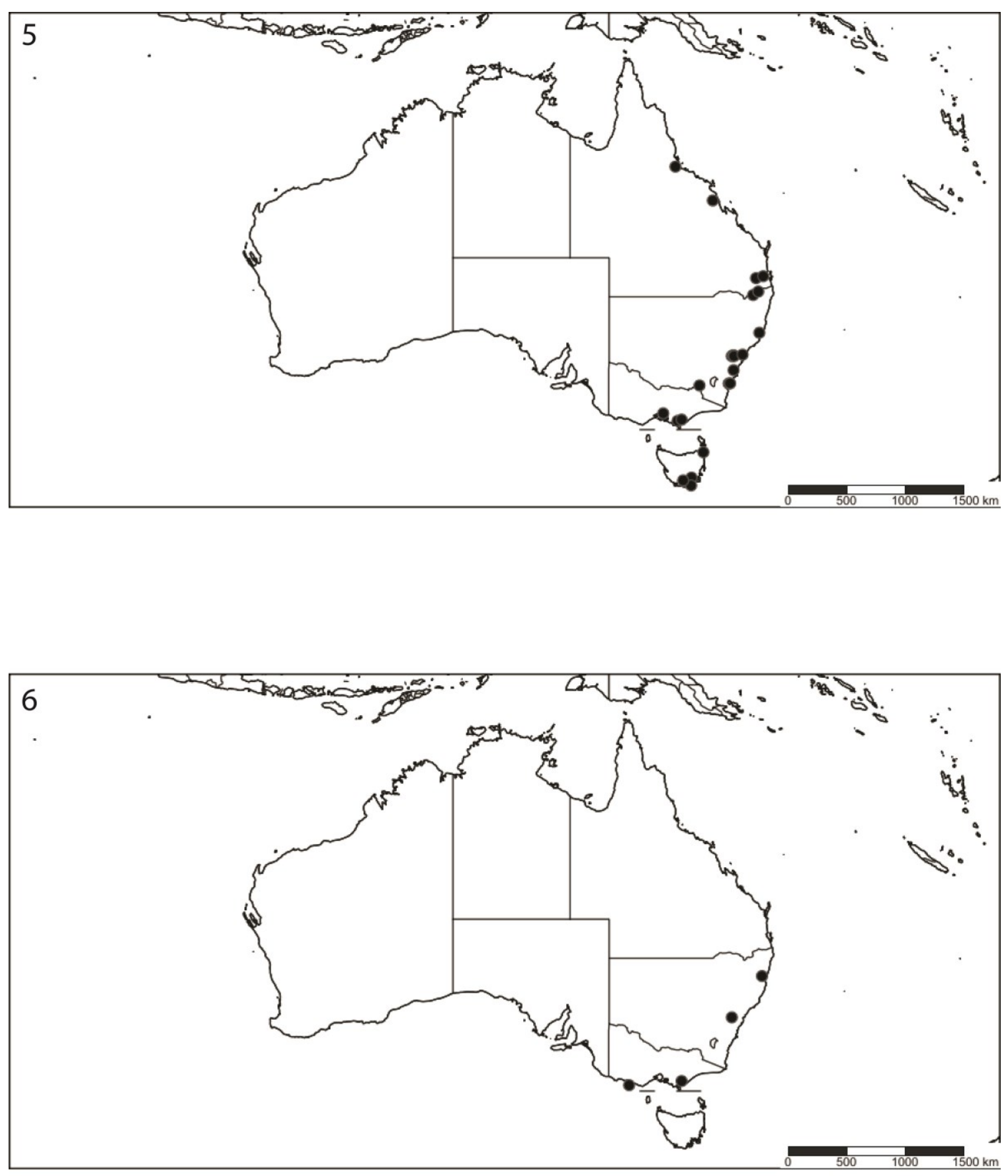

Chapter 3, Map 5: Psilota auripilosa

Chapter 3, Map 6: Psilota austropsilota 

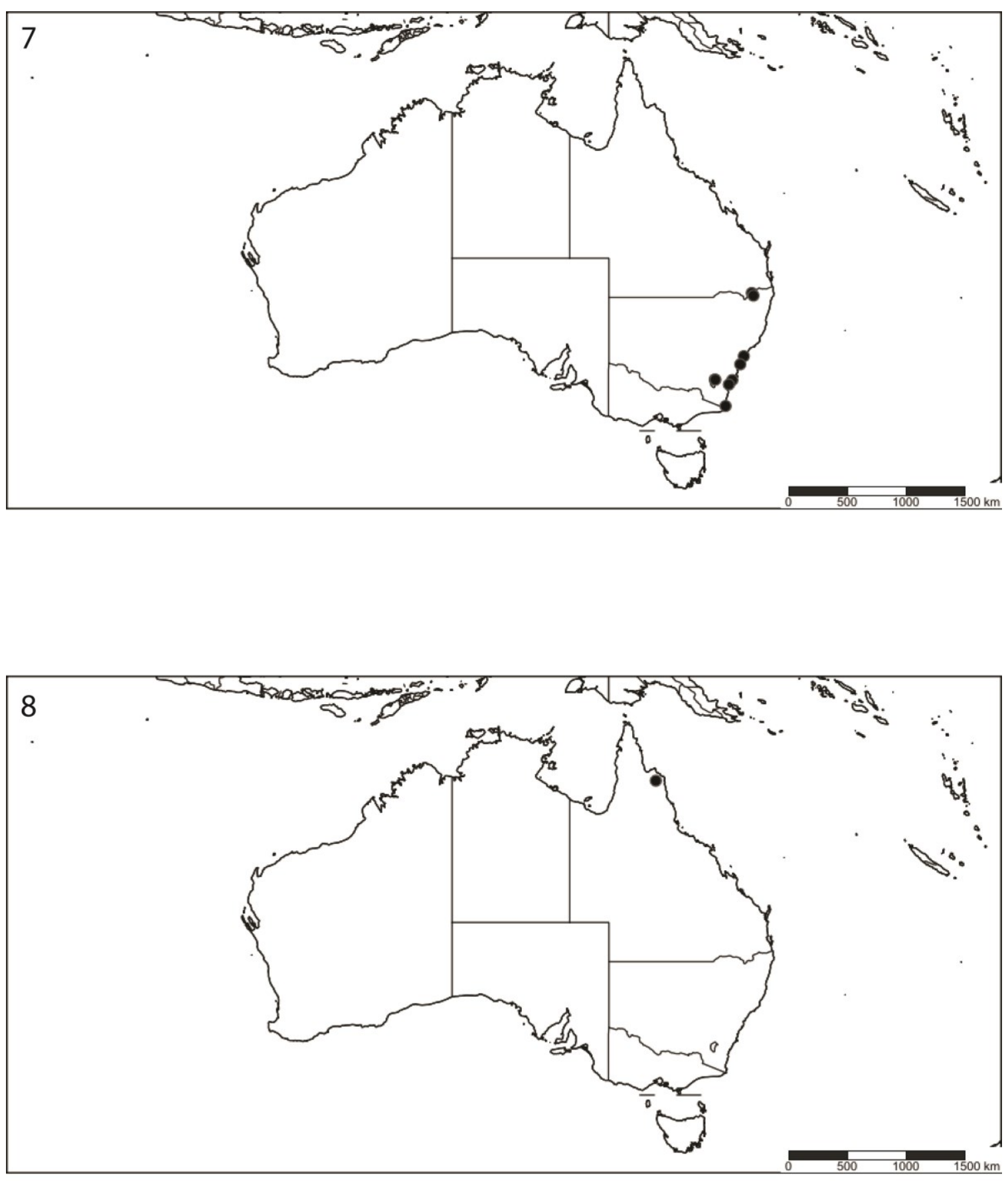

Chapter 3, Map 7: Psilota azurea

Chapter 3, Map 8: Psilota basalis 

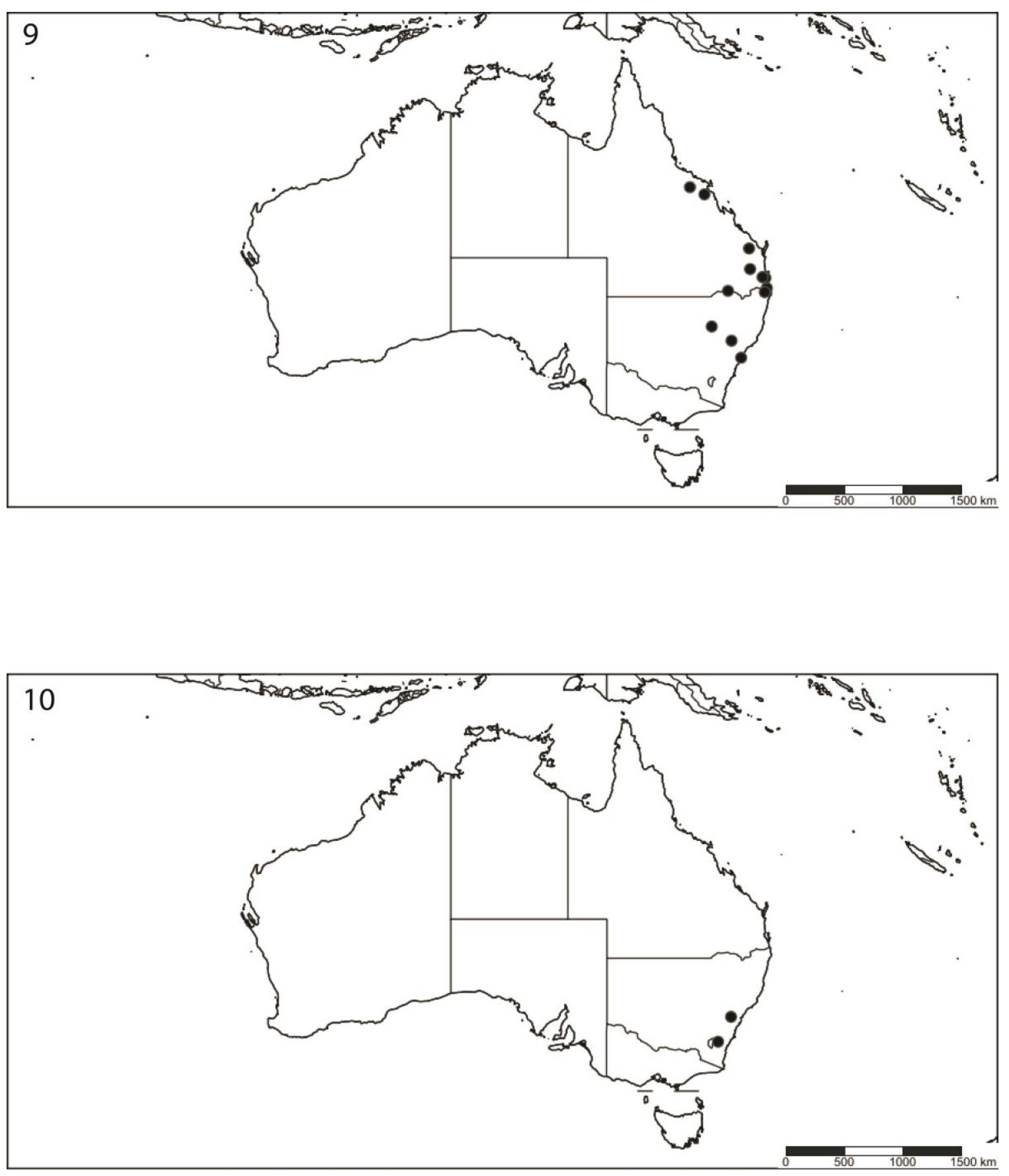

Chapter 3, Map 9: Psilota bicolor

Chapter 3, Map 10: Psilota brunnipennis 

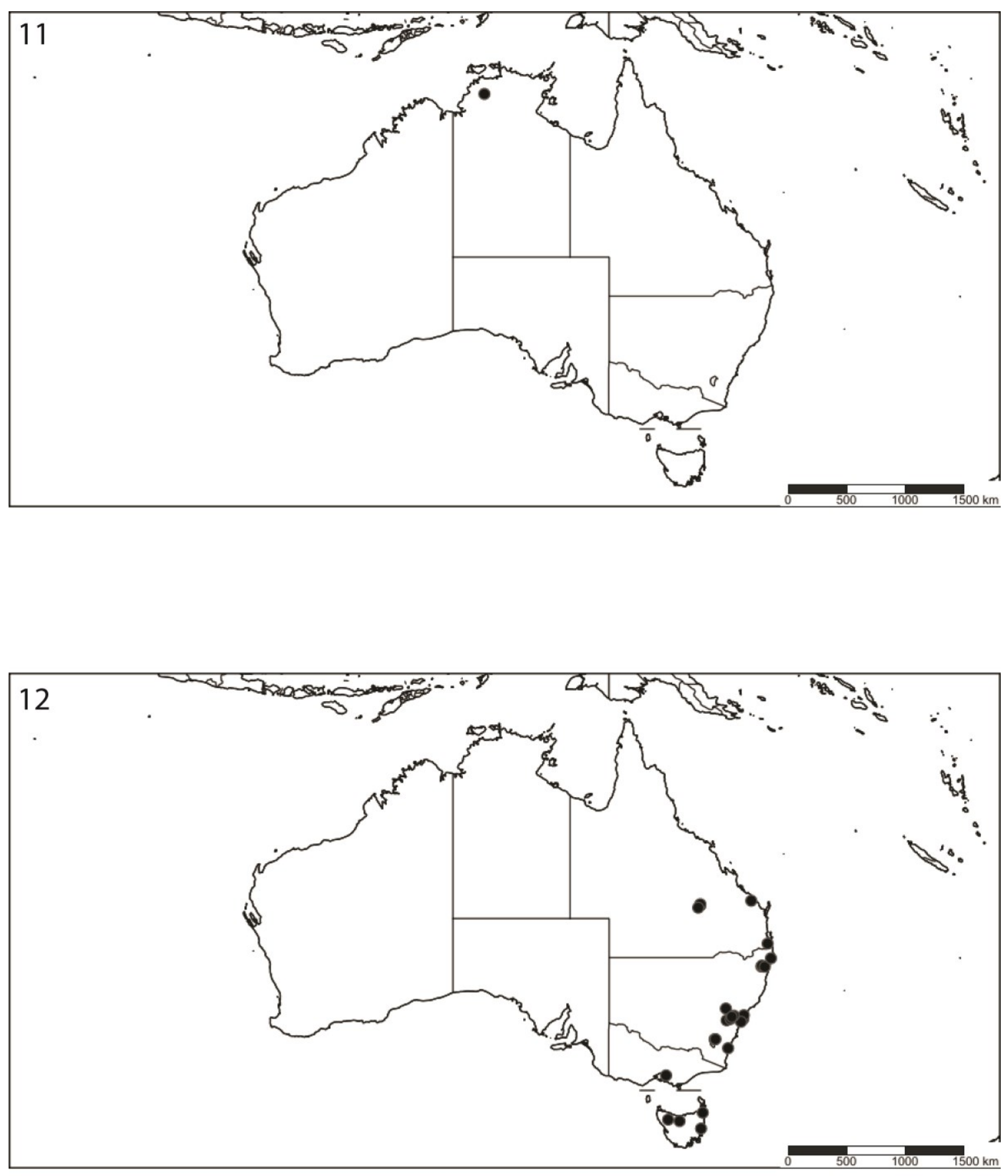

Chapter 3, Map 11: Psilota calva

Chapter 3, Map 12: Psilota coerulea 

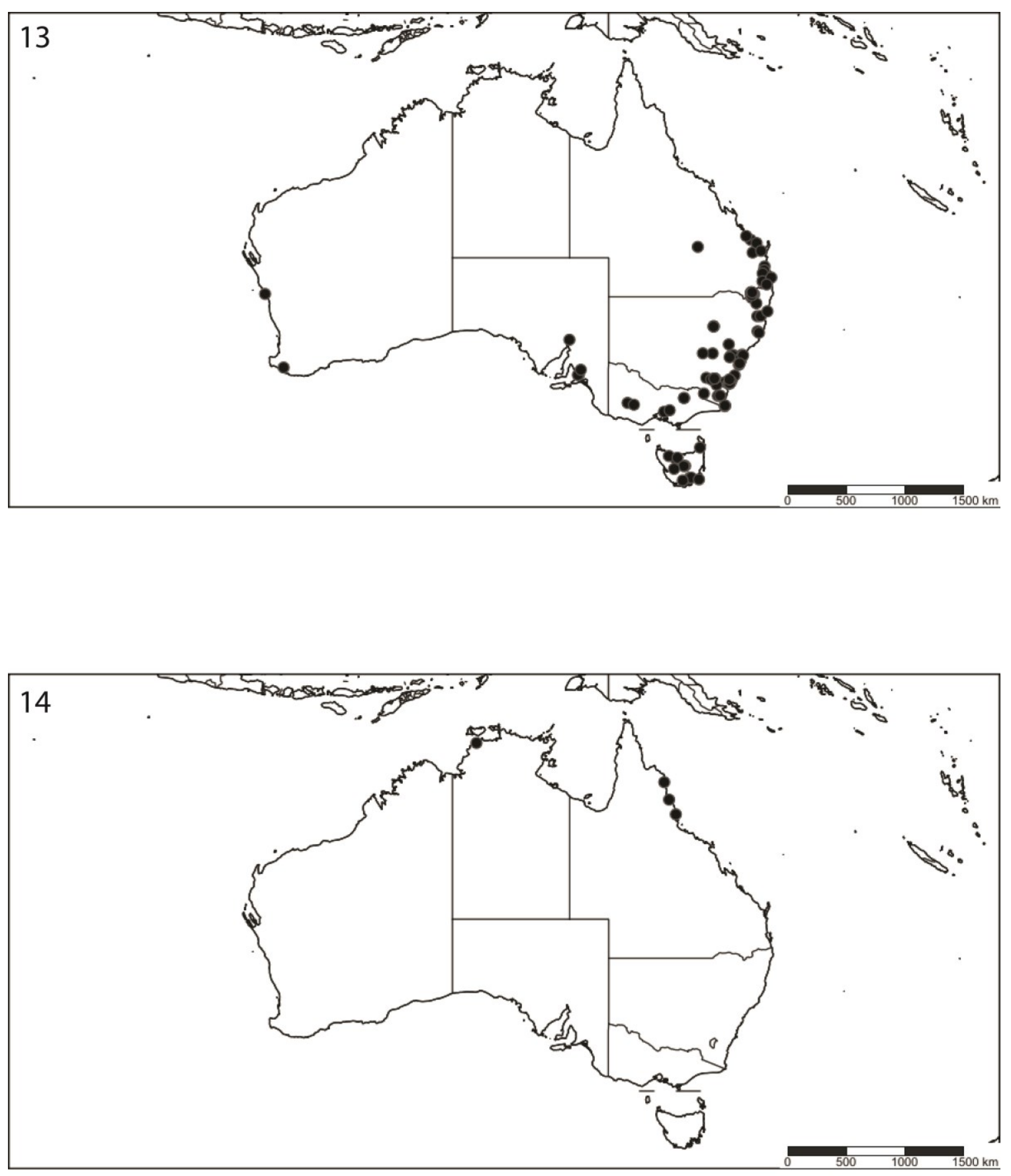

Chapter 3, Map 13: Psilota cupreus

Chapter 3, Map 14: Psilota darwini 

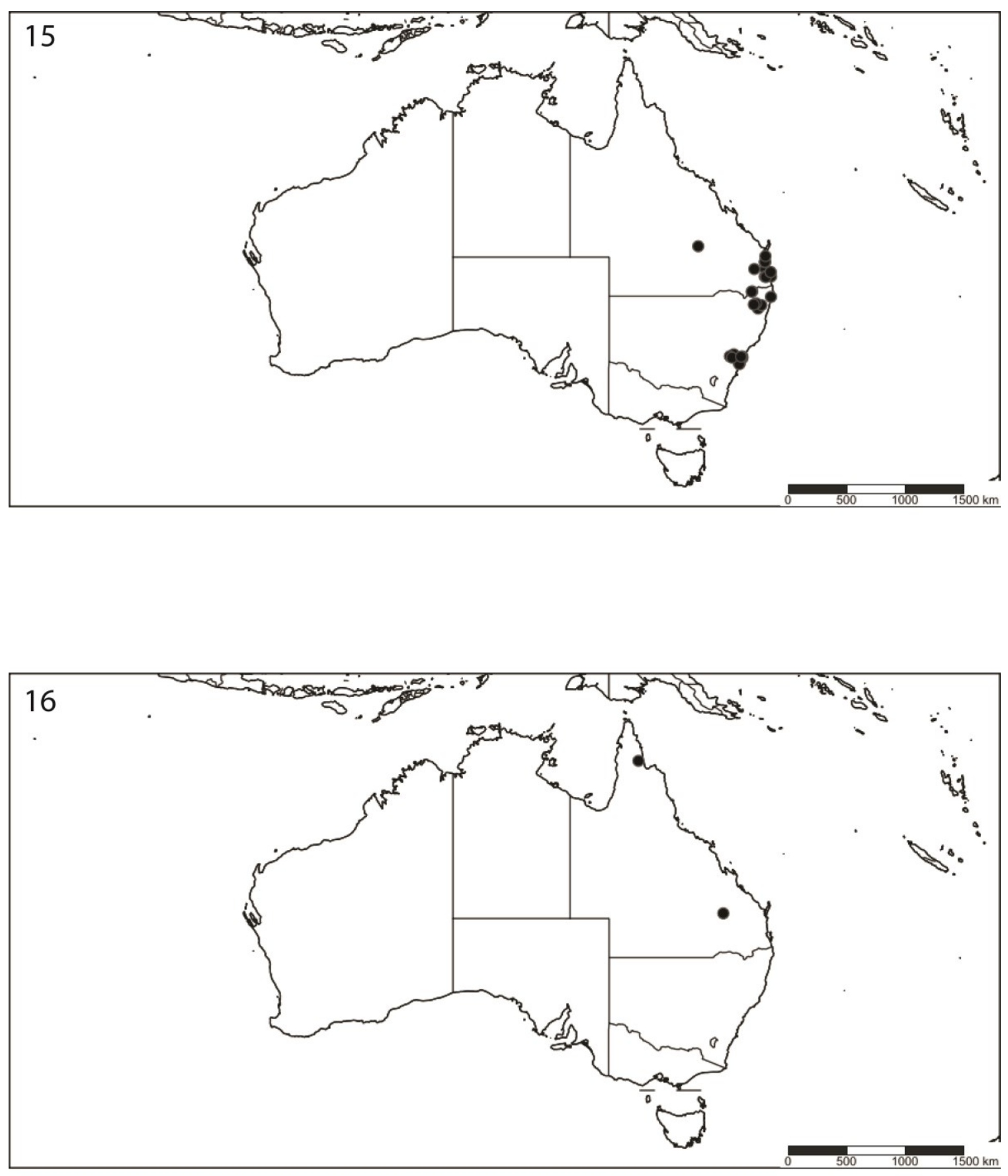

Chapter 3, Map 15: Psilota femoralis

Chapter 3, Map 16: Psilota livida 

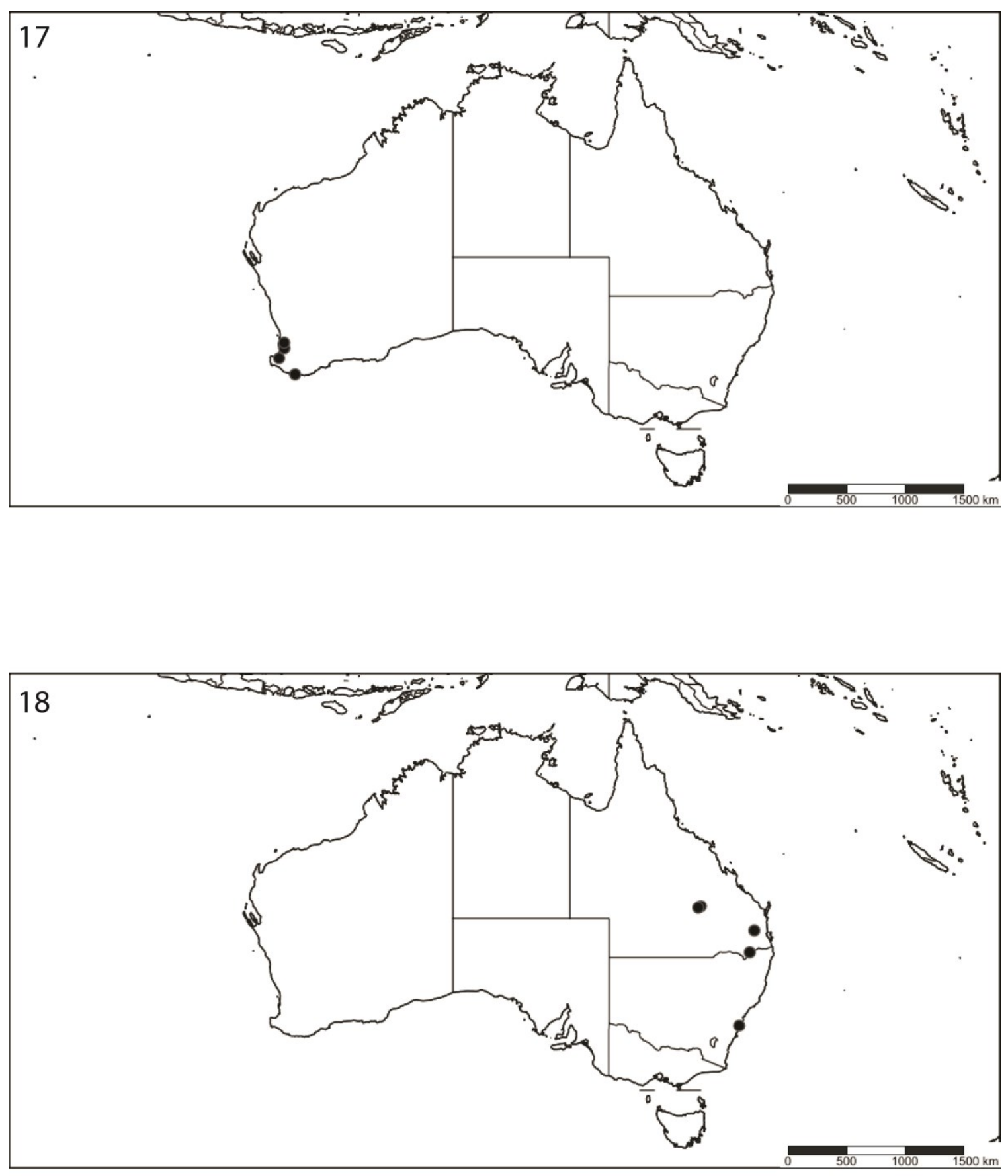

Chapter 3, Map 17: Psilota longipilosa

Chapter 3, Map 18: Psilota maculata 

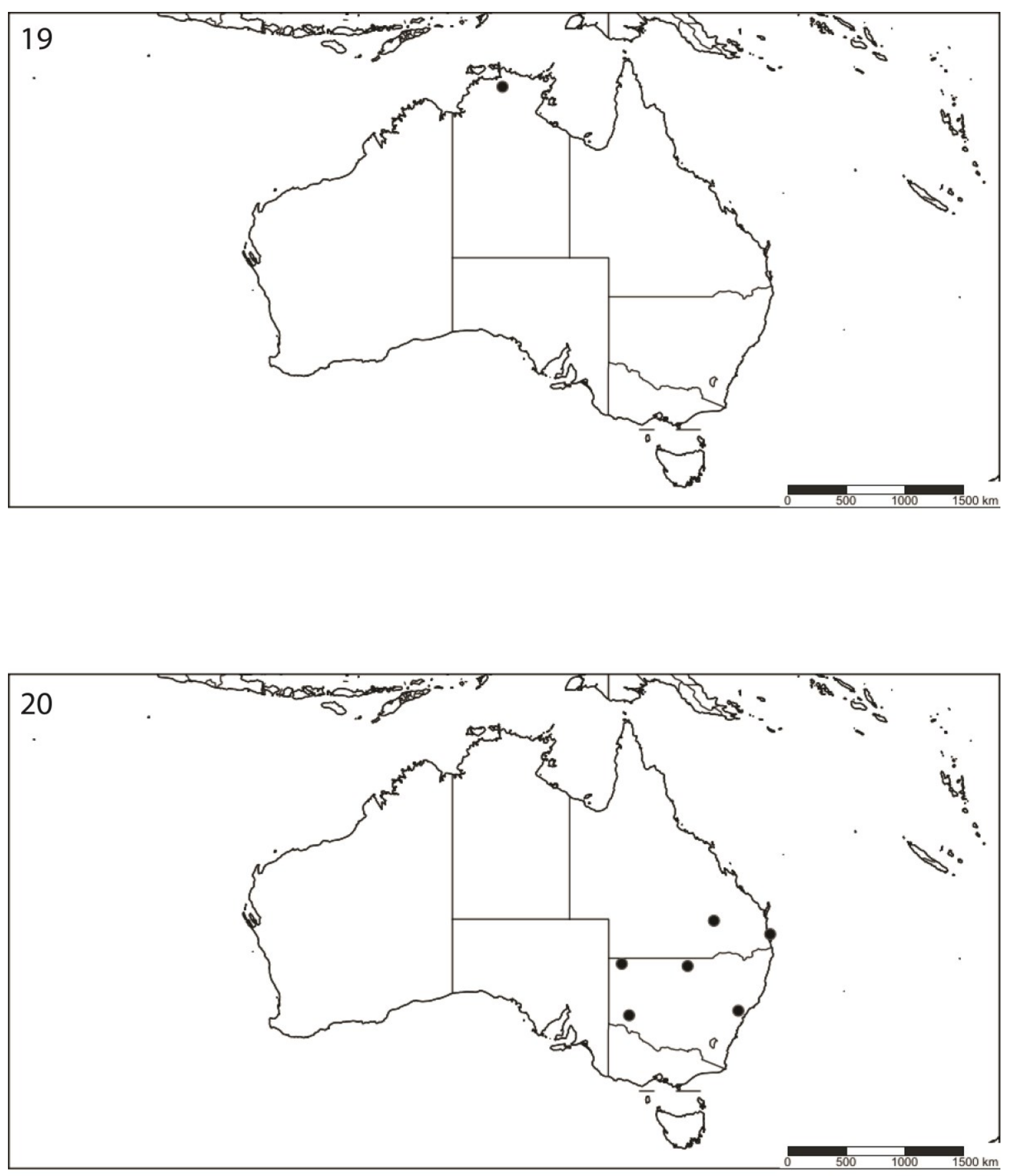

Chapter 3, Map 19: Psilota mcqueeni

Chapter 3, Map 20: Psilota metallica 

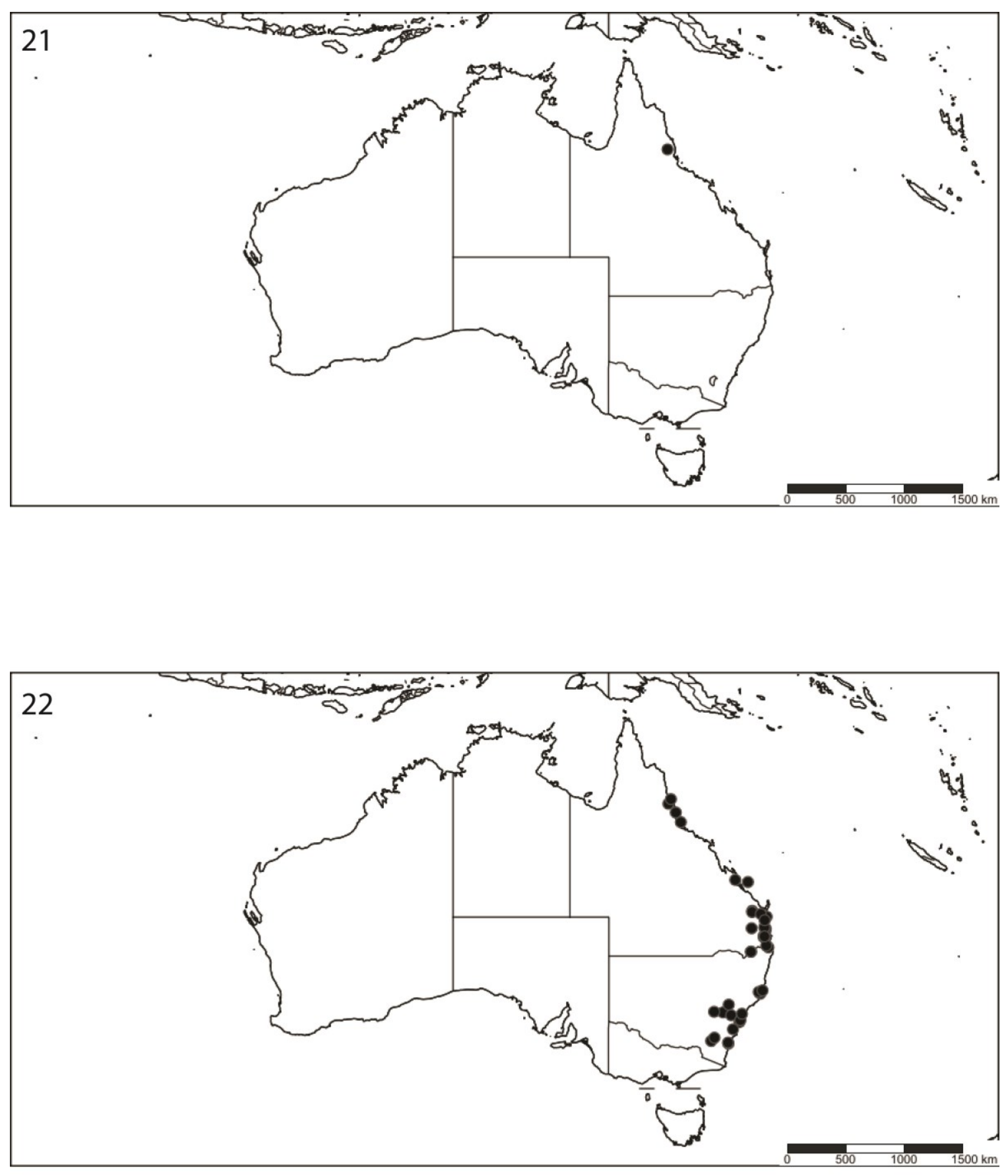

Chapter 3, Map 21: Psilota nigripilosa

Chapter 3, Map 22: Psilota nitida 

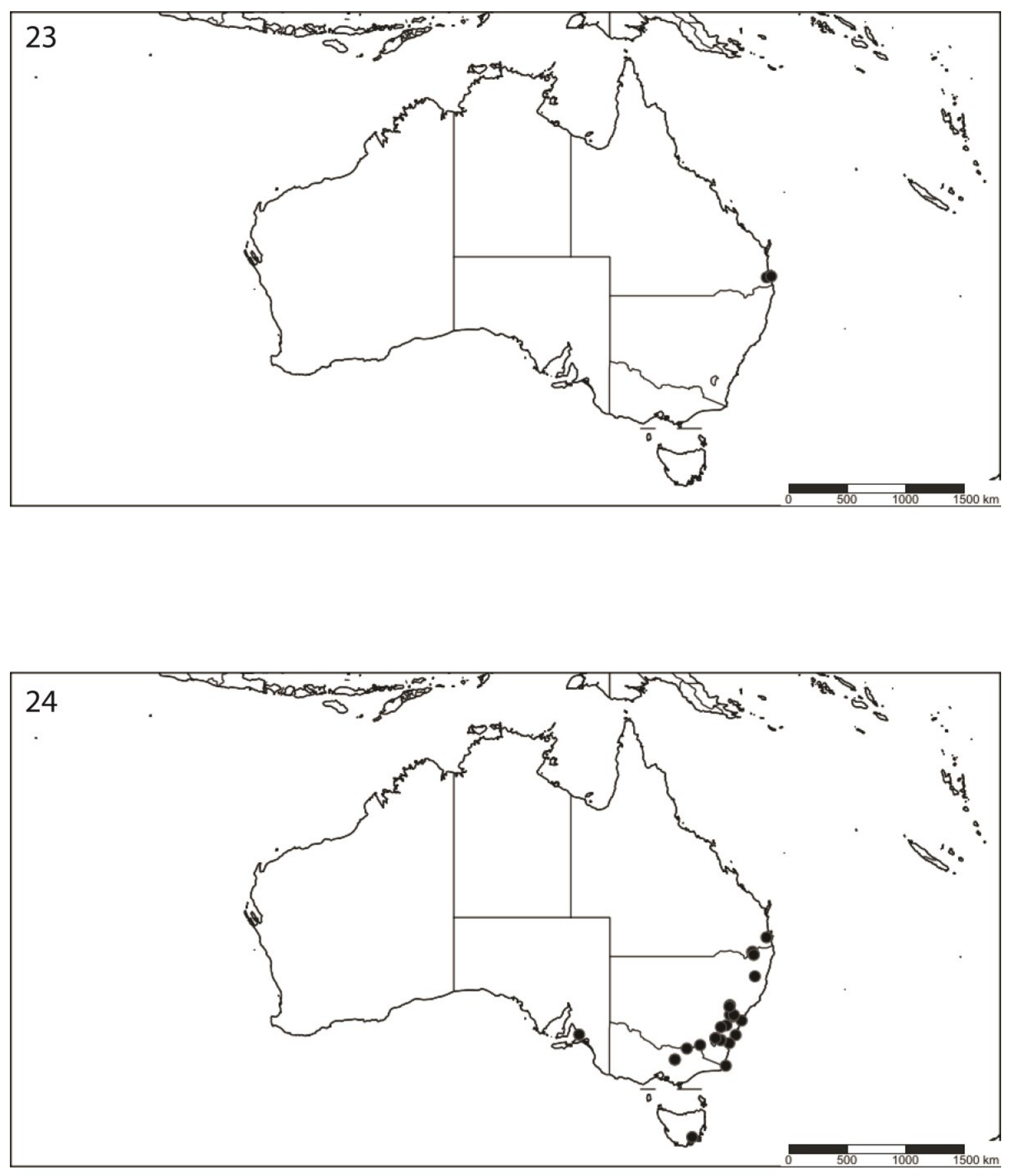

Chapter 3, Map 23: Psilota pallidifacies

Chapter 3, Map 24: Psilota pollinosa 

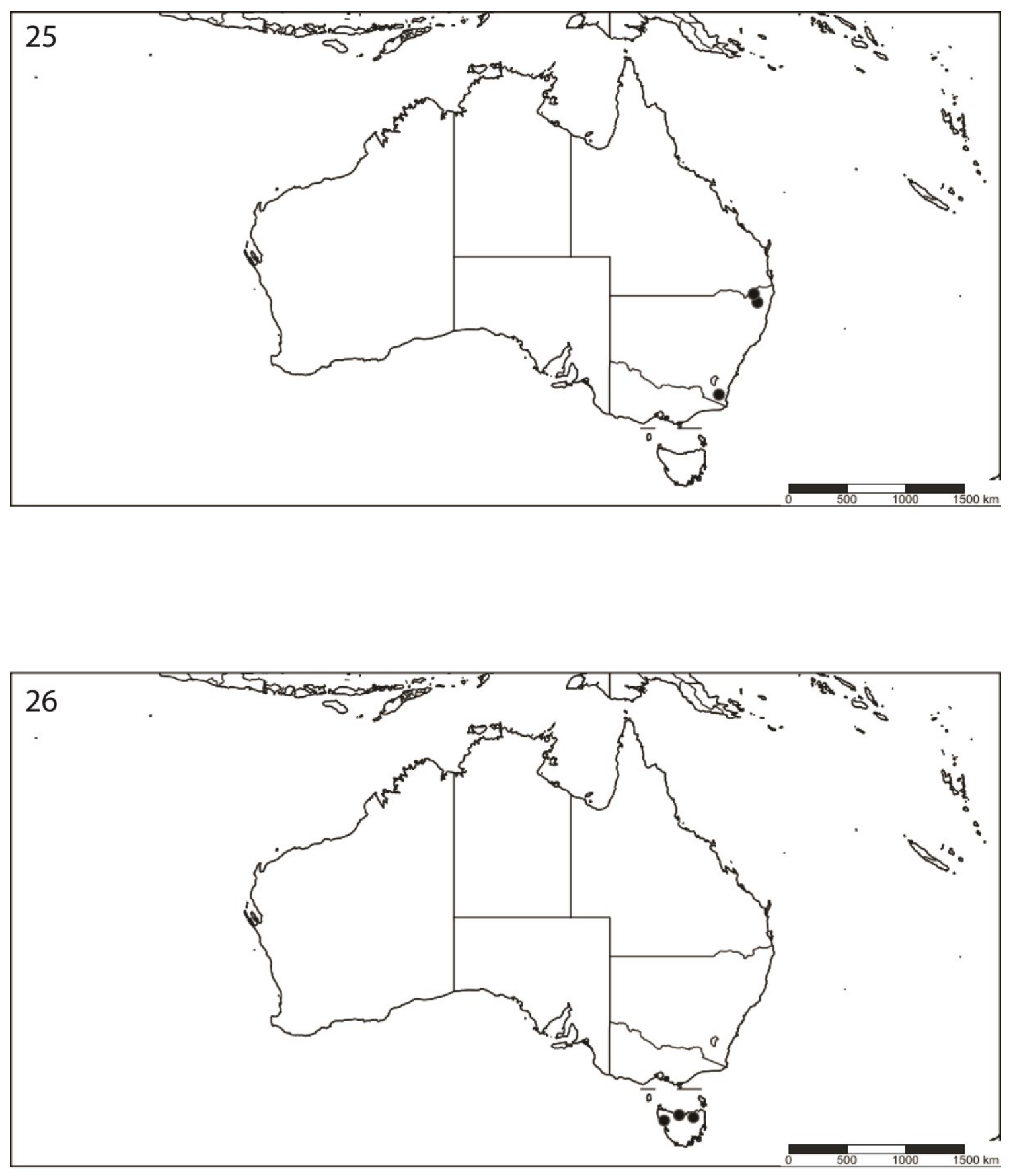

Chapter 3, Map 25: Psilota solata

Chapter 3, Map 26: Psilota spathistyla 

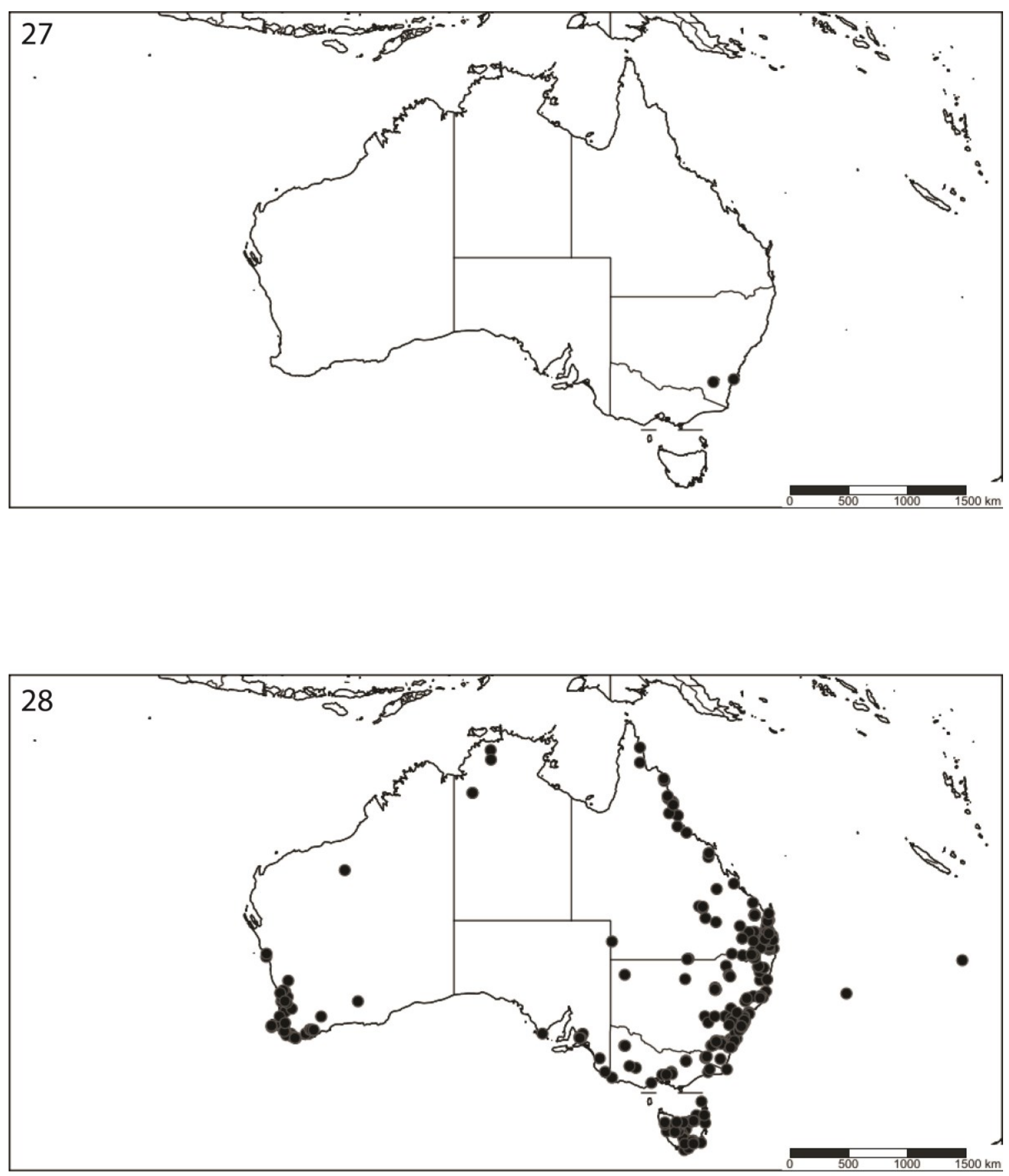

Chapter 3, Map 27: Psilota spinifemur

Chapter 3, Map 28: Psilota tristis 

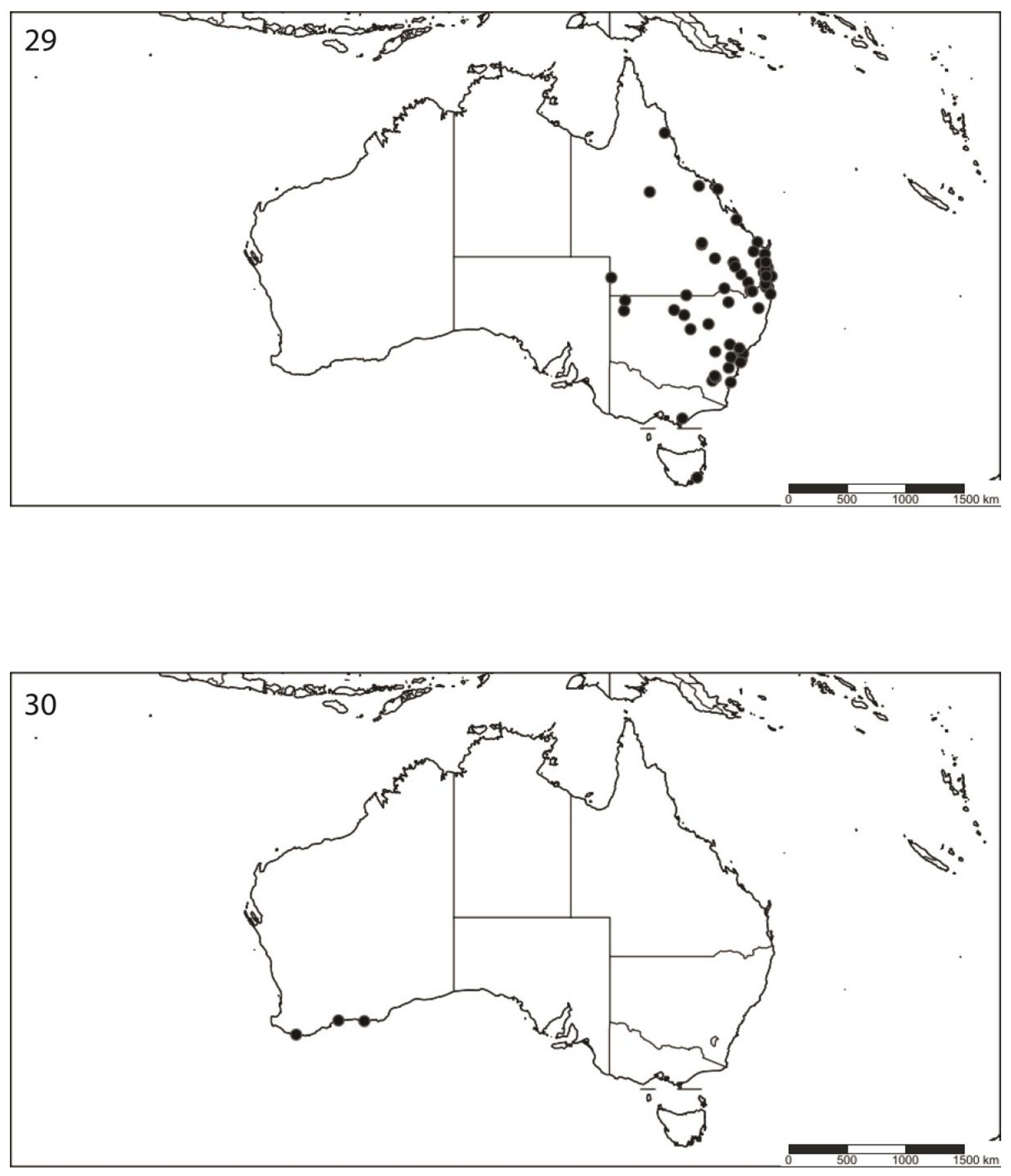

Chapter 3, Map 29: Psilota viridis

Chapter 3, Map 30: Psilota zophos 


\section{Conclusions and future directions}

Phylogenetic analyses from Chapters one and two returned results concordant with previous molecular phylogenies, with much higher support values. We hope the syrphid phylogeny in particular will act as a catalyst for future studies, both for syrphid-specific phylogenetic projects to investigate particular taxa in more detail, and for related Dipteran phylogenetic projects through the use of the AHE probe kit provided. Similarly, the Australian Psilota revision expanded our current knowledge of the genus, but the knowledge that at least two species complexes exist within the country merits further investigation.

We would like to further pursue the Psilota chapter in the near future. Many specimens within the P. tristis and P. cupreus species complexes are already on loan from Australian museums and have been DNA barcoded during the formulation of this thesis. Building on the current project and performing a more complete revision of the Australian Psilota should be relatively straightforward, but will require numerous genitalia dissections in order to find consistent, useful characters for discriminating between these closely related species. Even after these remaining species complexes are addressed, many Australian Psilota are known from very few specimens and nothing is known of their biology. Further fieldwork focused in the remote northern areas of the country is likely to uncover new species and provide rich natural history observations.

Further expanding the Psilota project to encompass the world fauna is an eventual goal. We know almost nothing about our local North American Psilota, other than that all known species actually represent species complexes. North 
American species are collected very rarely, resulting in a lack of fresh specimens for DNA work and dearth of specimen series from a single collecting locality useful for evaluating intraspecific morphological variation. As a result, the genus has thus far been intractable in North America. Our hope is that after revising the Australian fauna, a revisitation of the available North American material may prove fruitful with a better understanding of species-level morphological characters in the genus. A worldwide approach would also give us the benefit of examining the zoogeographic history of the genus. Currently, it is unknown whether Psilota originated in the Australian region, reached its peak diversity there, and spread outwards, or if Psilota evolved elsewhere, invaded Australia once or more, then diversified due to lack of niche competition. Although the absence of Psilota from South America suggests a secondary invasion of Australia, rather than a Gondwanan origin for the genus, a proper world phylogeny of the genus would be extremely elucidating.

Future phylogenetic research goals include a more densely sampled Syrphidae phylogeny (including world representation of Psilota species), as well as many other genera of Eristaline syrphid as possible, as tribal placement of Psilota is still unknown. The lower Cyclorrhapha phylogeny may also merit further examination and/or more extensive taxon sampling, as the lack of definitive placement of Lonchopteridae was disappointing. The same results, with Lonchopteridae switching placement in amino acid versus nucleotide analyses, was obtained in a recent transcriptomic analysis of Syrphoidea, suggesting these longstanding issues of Diptera classification will not be easily resolved. Similarly, 
reconciling molecular and morphological phylogenetics represents a substantial remaining task. The recent discovery of Puyehuemyia chandleri may ultimately shed light on the placement of Opetiidae, but before this happens male specimens must be collected for genital evaluation, and specimens will need to be collected into alcohol for molecular sequencing. Once again, further fieldwork is required in order to proceed phylogenetically. 